SAND2004-5216

Unlimited Release

Printed December 2004

\title{
Probability of Loss of Assured Safety in Temperature Dependent Systems with Multiple Weak and Strong Links
}

Jon C. Helton, Jay D. Johnson, and William L. Oberkampf

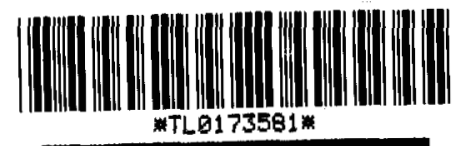

Prepared by Sandia National Laboratories

Albuquerque, New Mexico 87185 and Livermore, California 94550

SANDIA NATIONAL

LABORATORIES

TECHNICAL LIBRARY

Sandia is a multiprogram laboratory operated by Sandia Corporation, a Lockheed Martin Company, for the United States Department of Energy's National Nuclear Security Administration under Contract DE-AC04-94AL85000.

Approved for public release; further dissemination unlimited.

\section{Sandia National Laboratories}
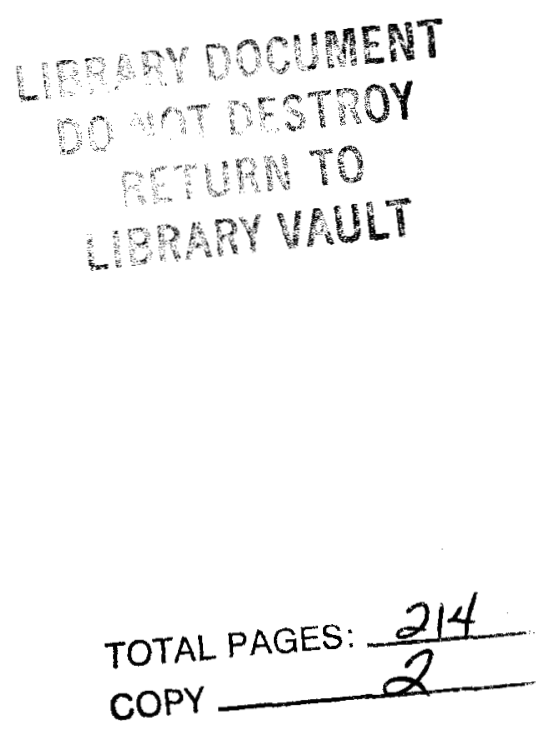
Issued by Sandia National Laboratories, operated for the United States Department of Energy by Sandia Corporation.

NOTICE: This report was prepared as an account of work sponsored by an agency of the United States Government. Neither the United States Government, nor any agency thereof, nor any of their employees, nor any of their contractors, subcontractors, or their employees, make any warranty, express or implied, or assume any legal liability or responsibility for the accuracy, completeness, or usefulness of any information, apparatus, product, or process disclosed, or represent that its use would not infringe privately owned rights. Reference herein to any specific commercial product, process, or service by trade name, trademark, manufacturer, or otherwise, does not necessarily constitute or imply its endorsement, recommendation, or favoring by the United States Government, any agency thereof, or any of their contractors or subcontractors. The views and opinions expressed herein do not necessarily state or reflect those of the United States Government, any agency thereof, or any of their contractors.

Printed in the United States of America. This report has been reproduced directly from the best available copy.

Available to DOE and DOE contractors from

U.S. Department of Energy

Office of Scientific and Technical Information

P.O. Box 62

Oak Ridge, TN 37831

Telephone: (865)576-8401

Facsimile: (865)576-5728

E-Mail: reports@adonis.osti.gov

Online ordering: http://www.osti.gov/bridge

Available to the public from

U.S. Department of Commerce

National Technical Information Service

5285 Port Royal Rd

Springfield, VA 22161

Telephone: (800)553-6847

Facsimile: (703)605-6900

E-Mail: orders@ntis.fedworld.gov

Online order: http://www.ntis.gov/help/ordermethods.asp?loc=7-4-0\#online

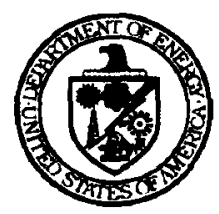


SAND2004-5216

Unlimited Release

Printed December 2004

\title{
Probability of Loss of Assured Safety in Temperature Dependent Systems with Multiple Weak and Strong Links
}

\author{
J.C. Helton \\ Department of Mathematics and Statistics \\ Arizona State University \\ Tempe, AZ 85287-1804 USA \\ J.D. Johnson \\ ProStat \\ Mesa, AZ 85204-5326 USA \\ W.L. Oberkampf \\ Sandia National Laboratories \\ Albuquerque, NM 87185-0779 USA
}

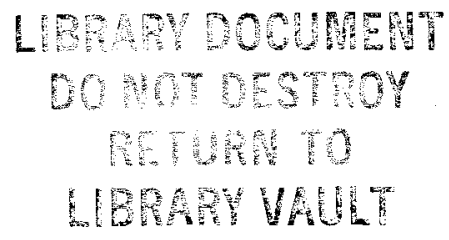

\begin{abstract}
Relationships to determine the probability that a weak link (WL)/strong link (SL) safety system will fail to function as intended in a fire environment are investigated. In the systems under study, failure of the WL system before failure of the SL system is intended to render the overall system inoperational and thus prevent the possible occurrence of accidents with potentially serious consequences. Formal developments of the probability that the WL system fails to deactivate the overall system before failure of the SL system (i.e., the probability of loss of assured safety, PLOAS) are presented for several WL/SL configurations: (i) one WL, one SL, (ii) multiple WLs, multiple SLs with failure of any SL before any WL constituting failure of the safety system, (iii) multiple WLs, multiple SLs with failure of all SLs before any WL constituting failure of the safety system, and (iv) multiple WLs, multiple SLs and multiple sublinks in each SL with failure of any sublink constituting failure of the associated SL and failure of all SLs before failure of any WL constituting failure of the safety system. The indicated probabilities derive from time-dependent temperatures in the WL/SL system and variability (i.e., aleatory uncertainty) in the temperatures at which the individual components of this system fail and are formally defined as multidimensional integrals. Numerical procedures based on quadrature (i.e., trapezoidal rule, Simpson's rule) and also on Monte Carlo techniques (i.e., simple random sampling, importance sampling) are described and illustrated for the evaluation of these integrals. Example uncertainty and sensitivity analyses for PLOAS involving the representation of uncertainty (i.e., epistemic uncertainty) with probability theory and also with evidence theory are presented.
\end{abstract}




\section{Acknowledgment}

Review provided at Sandia National Laboratories by T. Brown, J. Hobbs and C. Sallaberry. Editorial support provided by F. Puffer, J. Ripple and K. Best of Tech Reps, a division of Ktech Corporation.

\section{Dedication}

To Mike Bohn, whose interest in the reliability of time-dependent weak link/strong systems preceded our own and whose untimely death took both a friend and a professional colleague from us. 


\section{Contents}

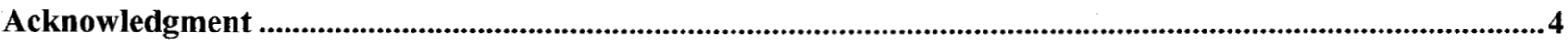

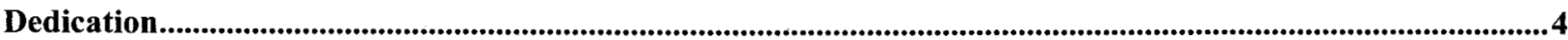

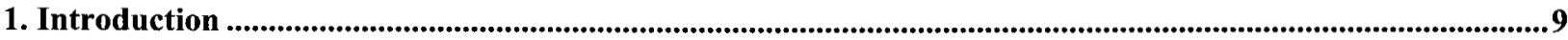

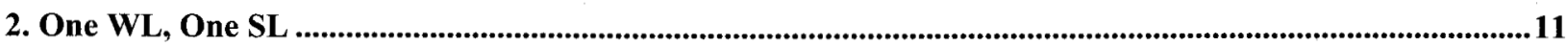

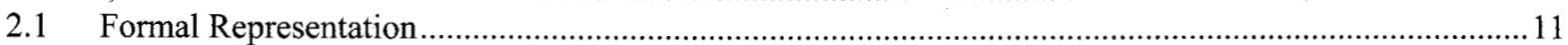

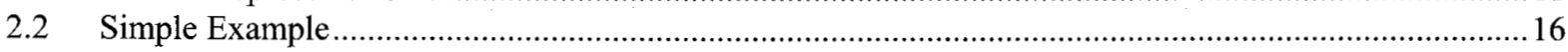

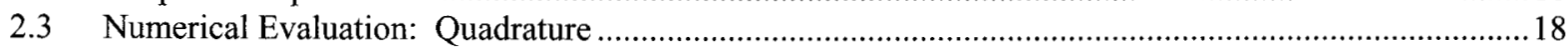

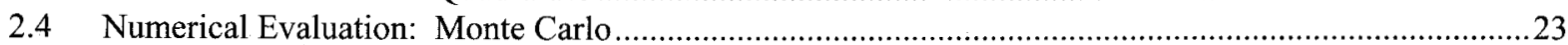

2.5 Numerical Evaluation: Simple Example............................................................................25

2.6 Numerical Evaluation: More Complex Example..........................................................................26

2.7 Comparison with One WL, One SL Representation Developed by Bohn.............................................30

3. Multiple WLs and SLs with Failure of One SL Before Any WL Constituting System Failure .....................33

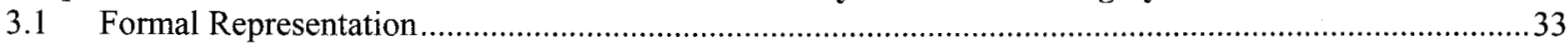

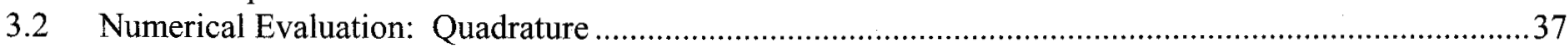

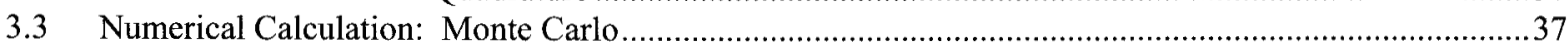

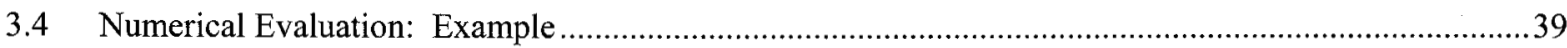

4. Multiple WLs and SLs with Failure of All SLs Before Any WL Constituting System Failure ......................43

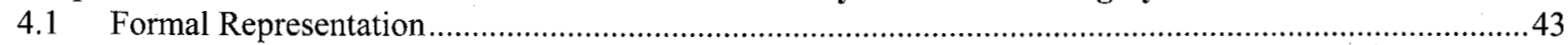

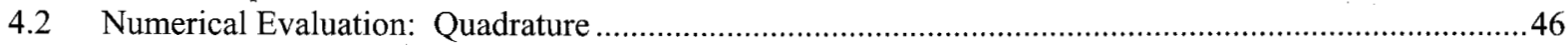

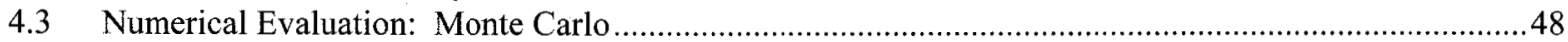

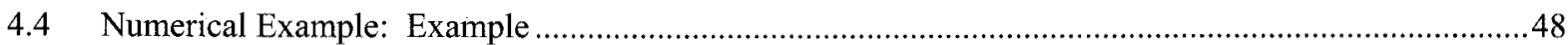

4.5 Comparison with One WL, Two SL Representation Developed by Bohn ............................................49

5. Multiple WLs and SLs with Individual SLs Composed of Multiple Components .......................................59

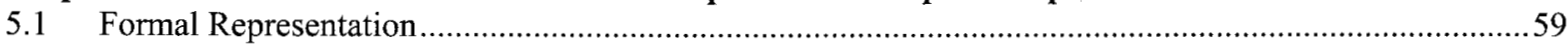

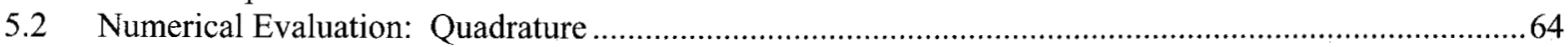

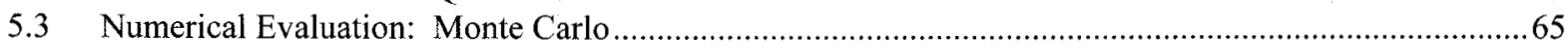

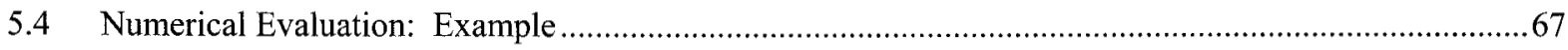

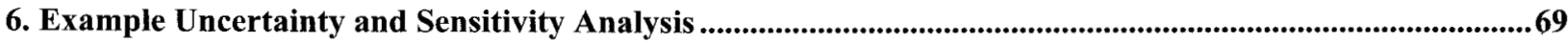

6.1 Representation of Uncertainty with Probability Theory …..................................................................69

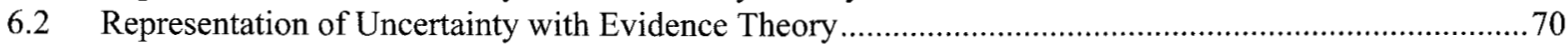

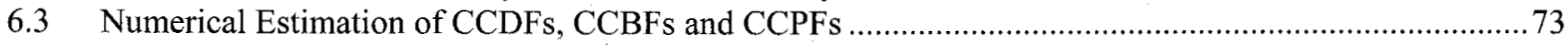

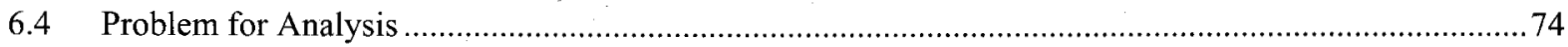

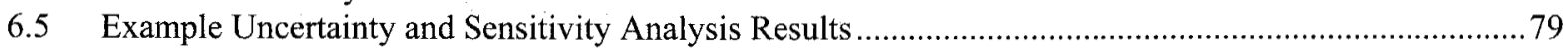

6.6 Justification of Assumptions for Estimation of CCBFs and CCPFs .................................................86

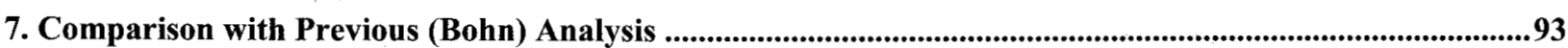

7.1 Bohn's Presentation for $\overline{p F}$ with Uncertainty in Temperature Curves...............................................93

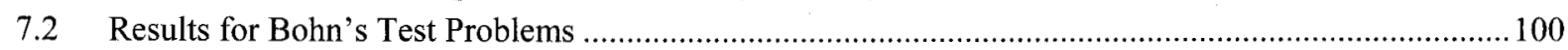

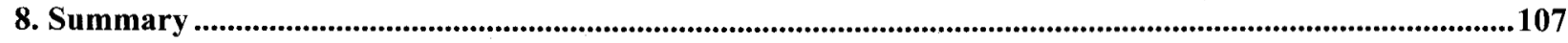

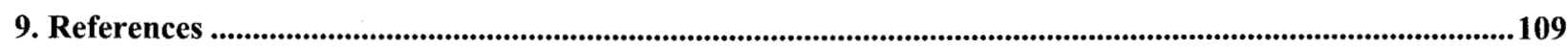

Appendix I: An Integral Formulation for Calculating Probabilities of Strong Link/Weak Link Cutsets...... I-1

Appendix II: P-RACE: A Program for Calculating Probabilities of Strong Link/Weak Link Cutsets ....... II-1

Appendix III: CPLOAS: A FORTRAN Program for the Calculation of the Probability of Loss of

Assured Safety ............................................................................................................................................................ 


\section{Figures}

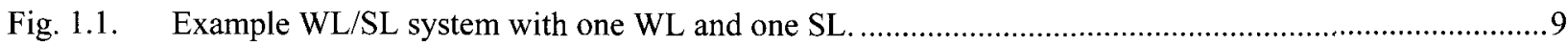

Fig. 2.1. Illustration of time dependent temperature curves for one WL and one SL....................................11

Fig. 2.2. Simple example illustrating calculation of failure probability $p F$ for one $\mathrm{WL}$ and one SL...................17

Fig. 2.3. Time-temperature curves defined in Eqs. (2.66) and (2.67) and used to illustrate calculation of

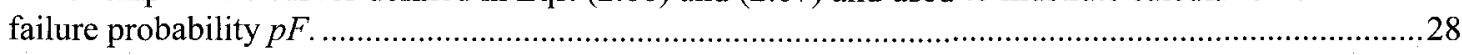

Fig. 2.4. Illustration of regions integrated over to determine the failure probability $p F$ with Eqs. (2.75) and

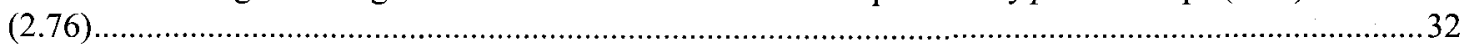

Fig. 3.1. Time-temperature curves defined in Eqs. (3.30) and (3.31) and used to illustrate calculation of failure probability $p F$ for two WLs and two SLs......

Fig. 4.1. Three-dimensional failure temperature regions used in example comparison with computational structure developed by M.P. Bohn (see App. I) for probability of loss of assured safety for system with one WL and two SLs.

Fig. 6.1. Graphical illustration of uncertainty information in Table 6.2 with variable range $[a, b]$ normalized

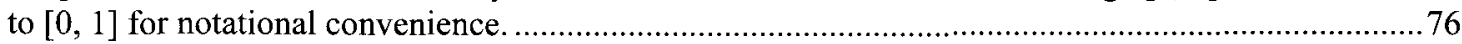

Fig. 6.2. Distribution of a variable over the interval $[a, b]$ derived from the information in Table $6.2 \ldots \ldots \ldots \ldots \ldots . . .77$

Fig. 6.3. Graphical illustration of the 13 sets in Table 6.3 assigned nonzero BPAs with variable range $[a, b]$

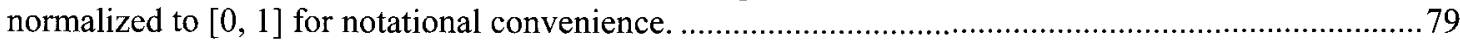

Fig. 6.4. Example WL/SL temperature curves and associated failure probability for sample element $\mathbf{x}_{144} \ldots \ldots \ldots . .80$

Fig. 6.5. Use of CCDF generated with a random sample of size 200 to display the epistemic uncertainty in the probability $p F$ that both SLs fail before either WL fails............................................................. 80

Fig. 6.6. Scatterplots for 16 variables in Table 6.1 sampled in uncertainty analysis of the probability $p F$ that

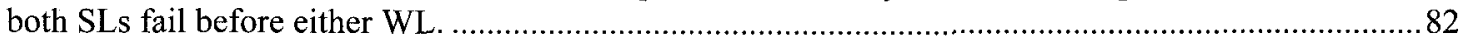

Fig. 6.7. Estimation of epistemic uncertainty in $p F$ with three replicated random samples: (a) $N=200$, (b) $N$ $=1000$, (c) $N=10,000$, and (d) one CCDF from each of the preceding frames. ................................. 83

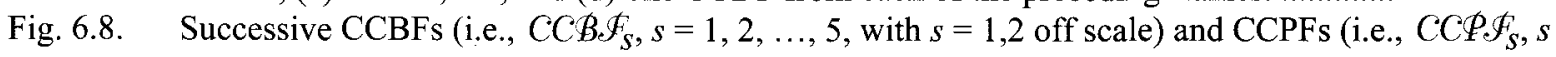
$=1,2, \ldots, 5$ ) estimated in application of algorithm described in Sect. 6.5 to develop an evidence theory representation of the epistemic uncertainty in the probability $p F$ that both SLs fail before either WL fails.

Fig. 6.9. Successive CCPFs (i.e., $C C P \oiint_{S}, s=1,2,3,4,5$ ) appearing in Fig. 6.8 plotted with shortened intervals on the axes..

\section{Tables}

Table 2.1. Approximations of Failure Probability $p F$ for System Defined in Sect. 2.2 with One WL, One SL and Uniform Distributions for WL and SL Failure Temperatures..

Table 2.2. Approximations of Failure Probability $p F$ for System Defined in Section 2.6 with One WL, One SL and Normal Distributions for WL and SL Failure Temperatures

Table 3.1. Approximation of Failure Probability $p F$ for System Defined in Sect. 3.4 with Two WLs, Two SLs, Normal Distributions for WL and SL Failure Temperatures, and Failure of a SL Before Either WL Constituting System Failure (i.e., the Failure Configuration Described in Sect. 3.1)...

Table 4.1. Approximation of Failure Probability $p F$ for System Defined in Sect. 3.4 with Two WLs, Two SLs, Normal Distributions for WL and SL Failure Temperatures, and Failure of Both SLs before Either WL Constituting System Failure (i.e., the Failure Configuration Described in Sect. 4.1) .....

Table 5.1. Approximation of Failure Probability $p F$ for System Defined in Sect. 5.4 with Two WLs, Two SLs, Two Components in Each SL, Normal Distributions for WL and SL Component Failure Temperatures, Failure of Either Component in a SL Constituting Failure of That SL, and Failure of Both SLs before Either WL Constituting System Failure (i.e., the Failure Configuration Described in Sect. 5.1).

Table 6.1. Uncertain Variables and Associated Uncertainty Ranges Considered in Example Uncertainty

Analyses. 
Table 6.2. Illustrative Specification of Uncertainty Information Used in Example Uncertainty Analyses with Probability Theory and Evidence Theory for Variables in Table 6.1

Table 6.3. Basic Probability Assignments (BPAs) for a Variable on the Interval $[a, b]$ Derived from the Information in Table 6.2

Table 6.4. Stepwise Regression Analysis with Log-Transformed and Rank-Transformed Data for Probability $p F$ that Both SLs Fail before Either WL Fails

Table 7.1. Summary of Representations Used for $\overline{p F}(t)$ in Bohn's Development for one WL and two SLs........101

Table 7.2. Three Test Problems Developed by Bohn for Use with P-RACE Program for Determination of Probability of Loss of Assured Safety (App. I) 102

Table 7.3. Comparison of $\overline{p F}(t)$ Obtained for Test Problems with Different Computational Procedures. .105 
This page intentionally left blank 


\section{Introduction}

Weak link (WL)/strong link (SL) systems constitute important parts of the operational design of high consequence systems. In such designs, the SL system is very robust and is intended to permit operation of the system under, and only under, intended conditions (e.g., by transmitting a command to activate the system). In contrast, the WL system is intended to fail in a predictable and irreversible manner under accident conditions (e.g., in the event of a fire) and render the entire system inoperational before an accidental operation of the SL system.

A simple example of a WL/SL system with one WL and one SL is shown in Fig. 1.1. Under nonoperational conditions, the WL is closed (e.g., permits the passage of an electrical signal) and the SL is open (e.g., does not permit the passage of an electrical signal) (Fig. 1.1a). For the entire system to operate, the SL must close and thereby allow the passage of an activating signal to the system (Fig. 1.1b). In the event of an accident, it is highly undesirable for the SL to close and place the system in a configuration in which it can be accidentally activated (Fig. 1.1b). To prevent the potential for such an accidental activation, the WL is designed to fail before the SL under accident conditions (Fig. 1.1c) and thus render impossible the passage of an activating signal should the SL fail at a subsequent time (Fig. 1.1d). As an aside, the phrase "WL failure," although widely used, is an oxymoron as such failure actually constitutes "WL success" in that the system has been deactivated by the intended (i.e., designed) operation of the WL.

As another example, the term WL is often applied to a device or component such as a capacitor. In an abnormal thermal environment, the capacitor is designed to melt and thus fail to function as a capacitor. Hence, the term WL failure. The WL as a device has indeed failed. However, the safety function of the WL has been a success.

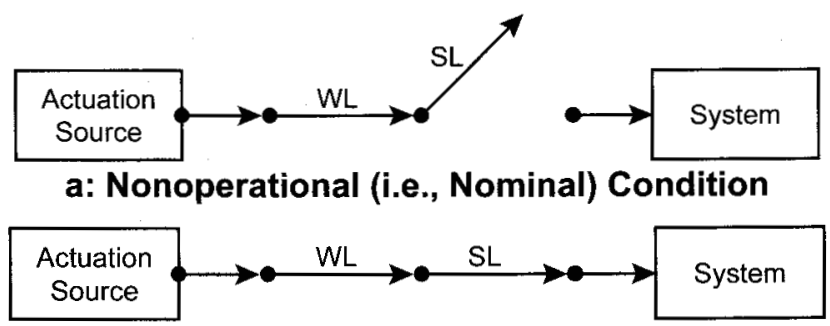

b: Operational Condition

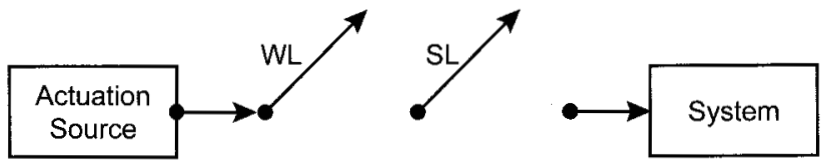

c: WL Failure before SL Closure

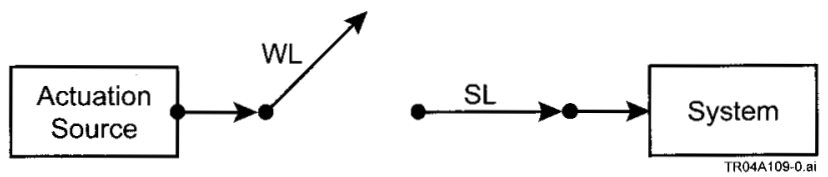

d: WL Failure with SL Closure

Fig. 1.1. Example WL/SL system with one WL and one SL. 
This presentation considers the behavior of WL/SL systems under fire conditions that arise from an accident. In particular, the overall system is assumed to be in a fire that causes heating of the WL and SL systems. The desired outcome in such an accident is for the WL system to fail before the SL system fails. The undesired outcome is for the SL system to fail before the WL system fails. The likelihood that the WL system fails to deactivate the entire system is referred to as the probability of loss of assured safety (PLOAS). The word "assured" appears in the preceding definition because failure of the WL system before the SL system renders the entire system inoperational but failure of the SL system before the WL system does not necessarily imply that the entire system will operate. Thus, PLOAS is not the probability that the system will operate; rather, it is the probability that the intended operation of the WL system fails to deactivate the system.

The determination of PLOAS falls within the broad area of study relating to the reliability of engineered systems (e.g., Refs. [1-7]). As developed in this presentation, the "probability" in PLOAS derives from variability in the temperatures at which individual WLs and SLs fail. The variability in WL and SL failure temperatures is assumed to be an aleatory uncertainty arising from manufacturing variability or some other source not explicitly included in the presented analyses (e.g., Refs. [8-11]). Because individual WLs and SLs have different failure temperatures and experience different time-dependent temperature regimes, there is in effect a race through time that determines whether the WLs fail before the SLs or the SLs fail before the WLs. Thus, the determination of PLOAS falls in the subarea of reliability analysis generally known as competing risk analysis or competing failure analysis (e.g., Refs. [12-15]).

Formal developments of the probability that the WL system fails to deactivate the overall system before failure of the SL system (i.e., PLOAS) are presented for several WL/SL configurations: (i) one WL, one SL (Sect. 2), (ii) multiple WLs, multiple SLs with failure of any SL before any WL constituting failure of the safety system (Sect. 3), (iii) multiple WLs, multiple SLs with failure of all SLs before any WL constituting failure of the safety system (Sect. 4), and (iv) multiple WLs, multiple SLs and multiple sublinks in each SL with failure of a sublink constituting failure of the associated SL and failure of all SLs before failure of any WL constituting failure of the safety system (Sect. 5). The indicated probabilities derive from time-dependent temperatures in the WL/SL system and variability in the temperatures at which the individual components of this system fail and are formally defined as multidimensional integrals. Numerical procedures based on quadrature (i.e., trapezoidal rule, Simpson's rule) and also on Monte Carlo techniques (i.e., simple random sampling, importance sampling) are described and illustrated for the evaluation of these integrals. Example uncertainty and sensitivity analyses for PLOAS involving the representation of uncertainty (i.e., epistemic uncertainty) with probability theory and also with evidence theory are presented (Sect. 6). A comparison with previous PLOAS results (Sect. 7) and a concluding discussion (Sect. 8) are also provided. Finally, appendices present an earlier approach to the determination of PLOAS (Apps. I, II) and software for determining PLOAS with the approaches developed in this presentation (App. III). 


\section{One WL, One SL}

The analysis of PLOAS for a system involving one WL and one SL is now presented. The following topics are considered: formal mathematical representation of PLOAS (Sect. 2.1), a simple illustrative example (Sect. 2.2), numerical evaluation of the defining integral for PLOAS with quadrature methods (Sect. 2.3), numerical evaluation of the defining integral for PLOAS with Monte Carlo methods (Sect. 2.4), a simple numerical example (Sect. 2.5), a more complex numerical example (Sect. 2.6), and comparison with an earlier representation for PLOAS developed by M.P. Bohn (Sect. 2.7, Apps. I, II).

\subsection{Formal Representation}

The system is assumed to involve one WL and one SL. The temperatures of the links are time dependent (Fig. 2.1 ) and are represented by

$T M P W L(t)=$ temperature $\left({ }^{\circ} \mathrm{C}\right)$ of $\mathrm{WL}$ at time $t(\min )$,

$\operatorname{TMPSL}(t)=$ temperature $\left({ }^{\circ} \mathrm{C}\right)$ of SL at time $t(\mathrm{~min})$.

Further, temperature of the SL is assumed to range from TMNSL to TMXSL, and time is assumed to range from tMIN to $t M A X$.

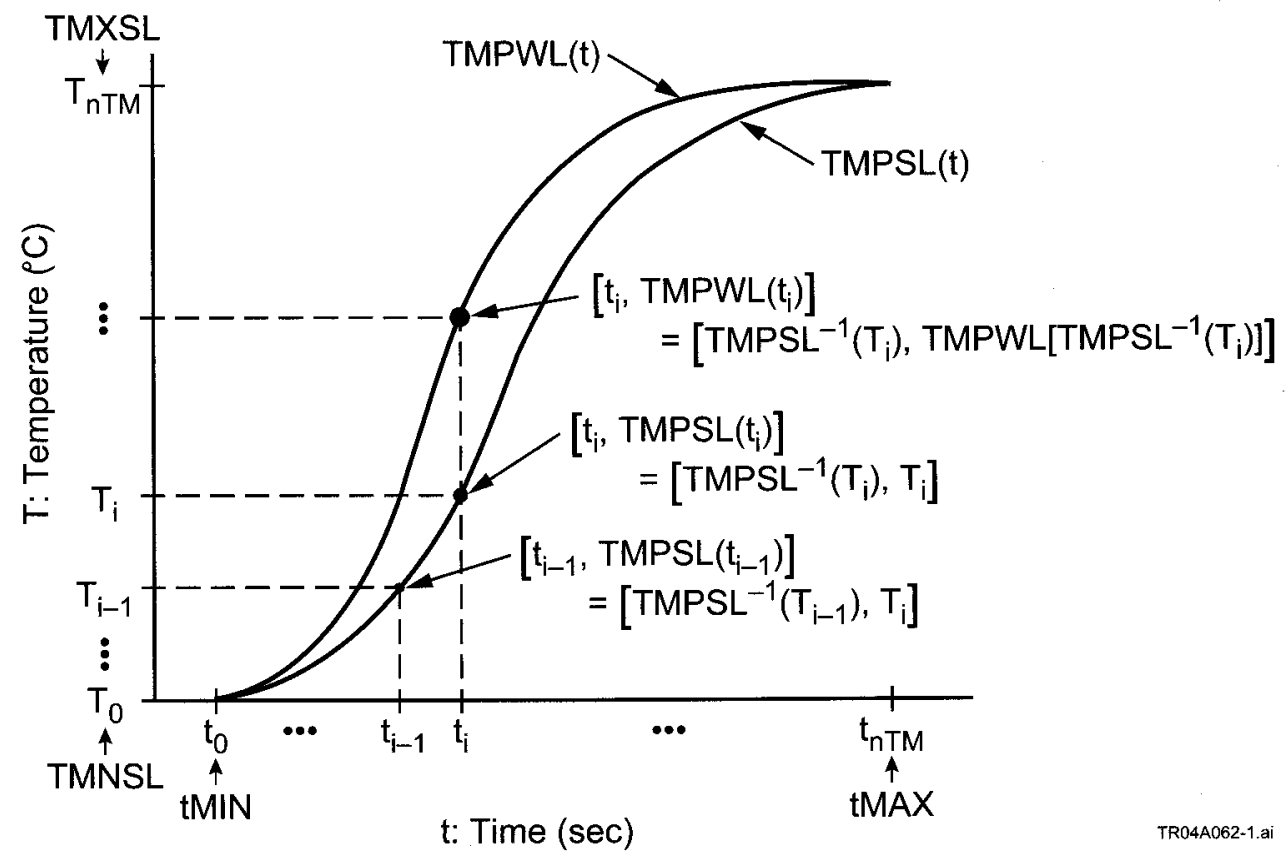

Fig. 2.1. Illustration of time dependent temperature curves for one $\mathrm{WL}$ and one SL. 
The preceding temperature functions are assumed to be strictly increasing with time (i.e., $T M P W L(t)<$ $\operatorname{TMPWL}(\tilde{t})$ and $\operatorname{TMPSL}(t)<\operatorname{TMPSL}(\tilde{t})$ for $t<\tilde{t})$. The following derivations are predicated on the assumption that the WL and the SL fail at the instant that they reach their failure temperatures. As a result, if $T M P W L(t)$ or $T M P S L(t)$ is non-increasing, then its value can be defined by linear interpolation between successive increasing local temperature maxima. This redefinition, if necessary, produces temperature functions with the appropriate increasing character.

The failure temperatures $T F W L$ and TFSL for the WL and SL, respectively, are assumed to be aleatory in the sense that the WL and SL are manufactured components and the exact failure temperature will vary from component to component. This variability is characterized by density functions $f W L$ and $f S L$, where

$$
\begin{aligned}
p W L(T 1, T 2) & =\text { probability that } T 1 \leq T F W L \leq T 2 \\
& =\int_{T 1}^{T 2} f W L\left(T_{W L}\right) \mathrm{d} T_{W L}, \\
\operatorname{pSL}(T 1, T 2) & =\text { probability that } T 1 \leq T F S L \leq T 2 \\
& =\int_{T 1}^{T 2} f S L\left(T_{S L}\right) \mathrm{d} T_{S L} .
\end{aligned}
$$

The density functions $f W L$ and $f S L$ define distributions on the ordinate of Fig. 2.1.

The objective of this section is to determine the probability $p F$ that the SL fails before the WL given $T M P W L(t), T M P S L(t), f W L\left(T_{W L}\right)$ and $f S L\left(T_{S L}\right)$. The sample space underlying this calculation is

$$
\mathcal{S}=\{\mathbf{t}: \mathbf{t}=[t F S L, t F W L]\},
$$

where $t F S L$ and $t F W L$ denote the failure times of the SL and WL, respectively. In particular,

$$
p F=\operatorname{prob}(\mathcal{E})
$$

where $\mathcal{E}$ is the subset of $\mathcal{S}$ defined by

$$
\mathcal{E}=\{\mathbf{t}: \mathbf{t}=[t F S L, t F W L] \in S \text { and } t F S L<t F W L\}
$$

and prob denotes probability.

The probability $p F$ will initially be obtained by an integration on time (i.e., on the abscissa of Fig. 2.1). Later, $p F$ will also be obtained by an integration on temperature (i.e., on the ordinate of Fig. 2.1). The set $\mathcal{E}$ can be represented by 


$$
\mathcal{E}=\bigcup_{i=1}^{n T M}\left(\varepsilon_{i} \cup \tilde{\varepsilon}_{i}\right)
$$

where

$$
\begin{aligned}
& \mathcal{E}_{i}=\left\{\mathbf{t}: \mathbf{t}=[t F S L, t F W L] \in \mathcal{E}, t_{i-1} \leq t F S L \leq t_{i}, t_{i}<t F W L\right\}, \\
& \tilde{\varepsilon}_{i}=\left\{\mathbf{t}: \mathbf{t}=[t F S L, t F W L] \in \mathcal{E}, t_{i-1} \leq t F S L \leq t_{i}, t F S L \leq t F W L \leq t_{i}\right\},
\end{aligned}
$$

and $t M I N=t_{0}<t_{1}<\ldots<t_{n T M}=t M A X$ is a partition of [tMIN, $\left.t M A X\right]$ as indicated in Fig. 2.1. The equality

$$
\operatorname{prob}(\varepsilon)=\sum_{i=1}^{n T M} \operatorname{prob}\left(\varepsilon_{i}\right)+\sum_{i=1}^{n T M} \operatorname{prob}\left(\tilde{\varepsilon}_{i}\right)
$$

holds because the sets $\varepsilon_{1}, \varepsilon_{2}, \ldots, \varepsilon_{n T M}, \tilde{\varepsilon}_{1}, \tilde{\varepsilon}_{2}, \ldots, \tilde{\varepsilon}_{n T M}$ are disjoint.

The following relations hold for $i=1,2, \ldots, n T M$ :

$$
\begin{aligned}
\operatorname{prob}\left(\tilde{\varepsilon}_{i}\right) \leq & I\left[\operatorname{TMPSL}\left(t_{i-1}\right), \operatorname{TMPSL}\left(t_{i}\right), f S L\right] I\left[\operatorname{TMPWL}\left(t_{i-1}\right), \operatorname{TMPWL}\left(t_{i}\right), f W L\right] \\
& \cong\left\{\left[\operatorname{TMPSL}\left(t_{i}\right)-\operatorname{TMPSL}\left(t_{i-1}\right)\right] f S L\left[\operatorname{TMPSL}\left(t_{i}\right)\right]\right\} \\
& \cdot\left\{\left[\operatorname{TMPWL}\left(t_{i}\right)-\operatorname{TMPWL}\left(t_{i-1}\right)\right] f W L\left[\operatorname{TMPWL}\left(t_{i}\right)\right]\right\} \\
& \leq B\left(\Delta t_{i}\right)^{2}
\end{aligned}
$$

and

$$
\begin{aligned}
\operatorname{prob}\left(\varepsilon_{i}\right) & =I\left[\operatorname{TMPSL}\left(t_{i-1}\right), \operatorname{TMPSL}\left(t_{i}\right), f S L\right] I\left[\operatorname{TMPWL}\left(t_{i}\right), \infty, f W L\right] \\
& \cong\left\{\left[\operatorname{TMPSL}\left(t_{i}\right)-\operatorname{TMPSL}\left(t_{i-1}\right)\right] f S L\left[\operatorname{TMPSL}\left(t_{i}\right)\right]\right\} I\left[\operatorname{TMPWL}\left(t_{i}\right), \infty, f W L\right],
\end{aligned}
$$

where the expression

$$
I[a, b, f]=\int_{a}^{b} f(v) d v
$$

appearing in Eqs. (2.9) and (2.10) represents the integral of the function $f$ from $a$ to $b$, and the constant $B$ appearing in Eq. (2.9) is independent of $i$ and derives from properties of the functions involved (i.e., TMPSL $(t)$ and TMPWL $(t)$ are continuous and of bounded variation on $[t M I N, t M A X]$ and $f S L\left(T_{S L}\right)$ and $f W L\left(T_{W L}\right)$ are bounded on [TMNSL, TMXSL]).

\section{As a result,}




$$
\begin{aligned}
\operatorname{prob}(\mathcal{E}) & =\lim _{n T M \rightarrow \infty} \sum_{i=1}^{n T M} \operatorname{prob}\left(\mathcal{E}_{i}\right)+\lim _{n T M \rightarrow \infty} \sum_{i=1}^{n T M} \operatorname{prob}\left(\tilde{\varepsilon}_{i}\right) \\
& =\lim _{n T M \rightarrow \infty} \sum_{i=1}^{n T M} \operatorname{prob}\left(\mathcal{\varepsilon}_{i}\right)+0 \\
& =\lim _{n T M \rightarrow \infty} \sum_{i=1}^{n T M}\left\{\left[\operatorname{TMPSL}\left(t_{i}\right)-\operatorname{TMPSL}\left(t_{i-1}\right)\right] \operatorname{fSL}\left[\operatorname{TMPSL}\left(t_{i}\right)\right]\right\} I\left[\operatorname{TMPWL}\left(t_{i}\right), \infty, f W L\right],
\end{aligned}
$$

where the first, second and third equalities follow from Eqs. (2.8), (2.9) and (2.10), respectively. Evaluation of the final limit in Eq. (2.11) leads to the representation of $\operatorname{prob}(\mathcal{E})$, and hence $p F$, by

$$
\begin{aligned}
p F & =\int_{t M I N}^{t M A X}\{f S L[T M P S L(t)]\}\left\{\int_{T M P W L(t)}^{\infty} f W L\left(T_{W L}\right) \mathrm{d} T_{W L}\right\} \mathrm{d} T M P S L(t) \\
& =\int_{t M I N}^{t M A X}\{f S L[T M P S L(t)]\}\{\mathrm{d} T M P S L(t) / \mathrm{d} t\}\left\{\int_{T M P W L(t)}^{\infty} f W L\left(T_{W L}\right) \mathrm{d} T_{W L}\right\} \mathrm{d} t
\end{aligned}
$$

where the first integral is a Riemann-Stieltjes integral (i.e., an integral of the form $\int_{a}^{b} f(t) \operatorname{dg}(t)$; see Sect. 29, Ref. [16]) and the second integral is the corresponding Riemann integral (i.e., an integral of the form $\int_{a}^{b} f(t) g^{\prime}(t) \mathrm{d} t$; see Theorem 29.8, p. 220, Ref. [16]). As indicated by the final summation in Eq. (2.11), the two integrals in Eq. (2.12) correspond to integrating along the abscissa (i.e., the time axis) in Fig. 2.1.

The failure probability $p F$ can also be obtained by integration on temperature. Similarly to Eq. (2.7), the set $\varepsilon$ defined in conjunction with Eq. (2.6) can be represented by

$$
\varepsilon=\bigcup_{i=1}^{n T M P}\left(\mathscr{F}_{i} \cup \tilde{F}_{i}\right)
$$

where

$$
\begin{aligned}
& \mathscr{F}_{i}=\left\{\mathbf{t}: \mathbf{t}=[t F S L, t F W L] \in \mathcal{E}, T_{M P S L}^{-1}\left(T_{i-1}\right) \leq t F S L \leq \operatorname{TMPSL}^{-1}\left(T_{i}\right), \operatorname{TMPSL}^{-1}\left(T_{i}\right)<t F W L\right\} \\
& \tilde{\mathscr{F}}_{i}=\left\{\mathbf{t}: \mathbf{t}=[t F S L, t F W L] \in \mathcal{E}, \operatorname{TMPSL}^{-1}\left(T_{i-1}\right) \leq t F S L \leq \operatorname{TMPSL}^{-1}\left(T_{i}\right), t F S L \leq t F W L \leq \operatorname{TMPSL}^{-1}\left(T_{i}\right)\right\},
\end{aligned}
$$

and $T M N S L=T_{0}<T_{1}<\ldots<T_{n T M P}=T M X S L$ is a partition of [TMNSL, TMXSL] as indicated in Fig. 2.1.

Now, with the same logic as used to produce Eq. (2.11), 


$$
\begin{aligned}
\operatorname{prob}(\mathcal{E}) & =\lim _{n T M P \rightarrow \infty} \sum_{i=1}^{n T M P} \operatorname{prob}\left(\mathscr{F}_{i}\right)+\lim _{n T M P \rightarrow \infty} \sum_{i=1}^{n T M P} \operatorname{prob}\left(\tilde{\mathscr{F}}_{i}\right) \\
& =\lim _{n T M P \rightarrow \infty} \sum_{i=1}^{n T M P} \operatorname{prob}\left(\mathscr{F}_{i}\right)+0 \\
& =\lim _{n T M P \rightarrow \infty} \sum_{i=1}^{n T M P}\left\{\left[T_{i}-T_{i-1}\right] f S L\left(T_{i}\right)\right\} I\left(T M P W L\left[T M P S L^{-1}\left(T_{i}\right)\right], \infty, f W L\right)
\end{aligned}
$$

where the first, second and third equalities follow from results analogous to those contained in Eqs. (2.8), (2.9) and (2.10). Evaluation of the final limit in Eq. (2.14) leads to the representation of $\operatorname{prob}(\mathcal{E})$, and hence $p F$, by

$$
p F=\int_{T M N S L}^{T M X S L}\left\{f S L\left(T_{S L}\right) \int_{F\left(T_{S L}\right)}^{\infty} f W L\left(T_{W L}\right) \mathrm{d} T_{W L}\right\} \mathrm{d} T_{S L}
$$

where

$$
F\left(T_{S L}\right)=T M P W L\left[\operatorname{TMPSL}^{-1}\left(T_{S L}\right)\right]
$$

is used for notational compactness. As indicated by the final summation in Eq. (2.14), the integral in Eq. (2.15) corresponds to integrating along the ordinate (i.e., the temperature axis) in Fig. 2.1.

The integrals in Eqs. (2.12) and (2.15) define the same probability $p F$ and do not require independent derivations. In particular, the integral in Eq. (2.15) can be obtained from the Riemann integral in Eq. (2.12) by a change of variables. As a reminder, the change of variables formula for integrals is

$$
\int_{g(a)}^{g(b)} f(g) \mathrm{d} g=\int_{a}^{b} f[g(t)][\mathrm{d} g(t) / \mathrm{d} t] \mathrm{d} t
$$

provided $f$ is continuous on $[g(a), g(b)]$ and $d g(t) / d t$ is continuous on $[a, b]$ (p. 558, Ref. [17]). Application of the preceding change of variables to Eq. (2.12) with $g(t)=T M P S L(t)$ yields

$$
\begin{aligned}
p F & =\int_{t M I N}^{t M A X}\{f S L[\operatorname{TMPSL}(t)]\}\{\mathrm{d} T M P S L(t) / \mathrm{d} t\} I[\operatorname{TMPWL}(t), \infty, f W L] \mathrm{d} t \\
& =\int_{t M I N}^{t M A X}\{f S L[\operatorname{TMPSL}(t)]\} I\left[T M P W L\left\{T M P S L^{-1}[\operatorname{TMPSL}(t)]\right\}, \infty, f W L\right]\{d \operatorname{TMPSL}(t) / \mathrm{d} t\} \mathrm{d} t \\
& =\int_{g(t M I N)}^{g(t M A X)} f S L(g) I\left[T M P W L\left\{T M P S L^{-1}(g)\right\}, \infty, f W L\right] \mathrm{d} g \\
& =\int_{T M N S L}^{T M X S L}\left\{f S L\left(T_{S L}\right) \int_{F\left(T_{S L}\right)}^{\infty} f W L\left(T_{W L}\right) \mathrm{d} T_{W L}\right\} \mathrm{d} T_{S L}
\end{aligned}
$$

where (i) the change of variables takes place at the third equality and (ii) the fourth equality is produced from the equalities 


$$
\begin{aligned}
& g(t M I N)=T M P S L(t M I N)=T M N S L \\
& g(t M A X)=T M P S L(t M A X)=T M X S L
\end{aligned}
$$

and the notational replacement of $g$ by $T_{S L}$ within the integral. Thus, the integral defining $p F$ in Eq. (2.15) can be obtained from the integral defining $p F$ in Eq. (2.12) by a change of variables.

The Riemann integral with respect to time that defines $p F$ in Eq. (2.12) has the drawback that it requires the evaluation of the derivative of the function $\operatorname{TMPSL}(t)$. The Riemann integral with respect to temperature that defines $p F$ in Eq. (2.15) has the drawback that it requires the evaluation of the inverse function $T M P S L^{-1}(T)$. In practice, the Riemann-Stieltjes integral with respect to time that defines $p F$ in Eq. (2.12) may be the easiest of the three integrals to evaluate numerically. The assumption that the temperature functions are strictly increasing in time is made so that inverse functions such as $T M P S L^{-1}(T)$ will be single valued. The representations for $p F$ in Eq. (2.12) do not involve inverse temperature functions and are valid under the weaker assumption that $T M P W L(t)$ and TMPSL $(t)$ are nondecreasing functions of time.

\subsection{Simple Example}

A simple, illustrative example is now presented. In this example, $t M I N=0 \mathrm{~min}, t M A X=500 \mathrm{~min}, T M N S L=$ $100^{\circ} \mathrm{C}, T M X S L=1150^{\circ} \mathrm{C}, T F W L$ is uniform on $\left[200,600^{\circ} \mathrm{C}\right], T F S L$ is uniform on $\left[500,1000^{\circ} \mathrm{C}\right]$ and the temperature functions for the WL and SL are defined by

$$
T M P W L(t)=100+2 t
$$

and

$$
\operatorname{TMPSL}(t)=100+2.1 t
$$

for $0 \leq t \leq 500 \mathrm{~min}$ (see Fig. 2.2).

Additionally, the inverse functions associated with $T M P W L(t)$ and $T M P S L(t)$ are given by

$$
T M P W L^{-1}(T)=(T-100) / 2
$$

and

$$
\operatorname{TMPSL}^{-1}(T)=(T-100) / 2.1
$$

and the density functions associated with $T F W L$ and $T F S L$ are given by 


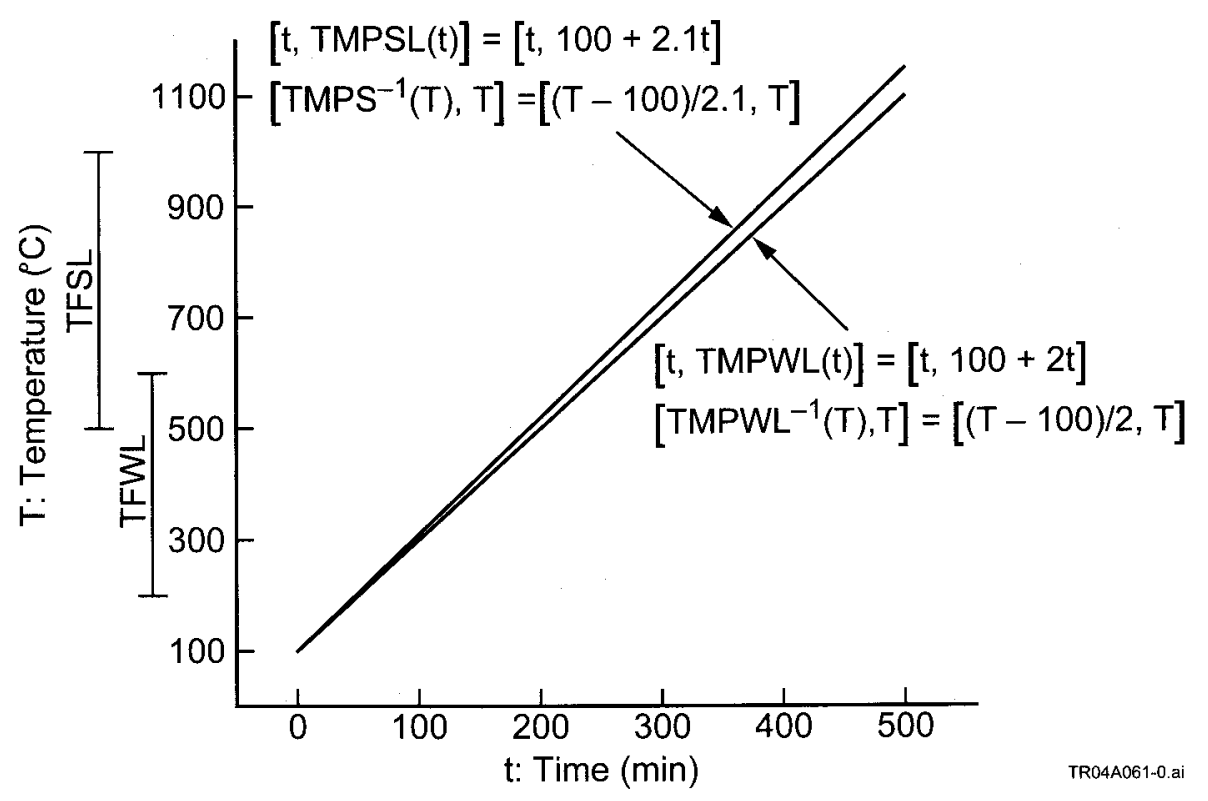

Fig. 2.2. Simple example illustrating calculation of failure probability $p F$ for one WL and one SL.

$$
f W L\left(T_{W L}\right)= \begin{cases}1 / 400 & \text { for } 200 \leq T_{W L} \leq 600^{\circ} \mathrm{C} \\ 0 & \text { otherwise }\end{cases}
$$

and

$$
f S L\left(T_{S L}\right)= \begin{cases}1 / 500 & \text { for } 500 \leq T_{S L} \leq 1000^{\circ} \mathrm{C} \\ 0 & \text { otherwise. }\end{cases}
$$

Finally, $F\left(T_{S L}\right)$ and $F^{-1}\left(T_{W L}\right)$ are defined by

$$
\begin{aligned}
F\left(T_{S L}\right) & =T M P W L\left[\operatorname{TMPSL}^{-1}\left(T_{S L}\right)\right] \\
& =100+2\left[\left(T_{S L}-100\right) / 2.1\right] \\
& =\left(10+2 T_{S L}\right) / 2.1
\end{aligned}
$$

and

$$
\begin{aligned}
F^{-1}\left(T_{W L}\right) & =\operatorname{TMPSL}\left[\operatorname{TMPWL}^{-1}\left(T_{W L}\right)\right] \\
& =100+2.1\left[\left(T_{W L}-100\right) / 2\right] \\
& =\left(2.1 T_{W L}-10\right) / 2
\end{aligned}
$$

and are used in defining limits of integration (see Eq. (2.15)).

The determination of $p F$ by integration on time as indicated in Eq. (2.12) is considered first. Specifically, 


$$
\begin{aligned}
p F & =\int_{t M I N}^{t M A X}\{f S L[\operatorname{TMPSL}(t)]\}\{\mathrm{d} \operatorname{TMPSL}(t) / \mathrm{d} t\}\left\{\int_{T M P W L(t)}^{\infty} f W L\left(T_{W L}\right) \mathrm{d} T_{W L}\right\} \mathrm{d} t \\
& =\int_{0}^{500}\{f S L[100+2.1 t]\}\{2.1\}\left\{\int_{100+2 t}^{\infty} f W L\left(T_{W L}\right) \mathrm{d} T_{W L}\right\} \mathrm{d} t \\
& =(2.1) \int_{(500-100) / 2.1}^{(1000-100) / 2.1}(1 / 500)\left\{\int_{100+2 t}^{\infty} f W L\left(T_{W L}\right) \mathrm{d} T_{W L}\right\} \mathrm{d} t \\
& =(2.1 / 500) \int_{400 / 2.1}^{250}\left\{\int_{100+2 t}^{600}(1 / 400) \mathrm{d} T_{W L}\right\} \mathrm{d} t \\
& \vdots \\
& =0.0372
\end{aligned}
$$

with the omitted steps involving elementary calculus manipulations.

The determination of $p F$ by integration on temperature as indicated in Eq. (2.17) is now considered. In this case,

$$
\begin{aligned}
p F & =\int_{T M N S L}^{T M X S L}\left\{f S L\left(T_{S L}\right) \int_{F\left(T_{S L}\right)}^{\infty} f W L\left(T_{W L}\right) \mathrm{d} T_{W L}\right\} \mathrm{d} T_{S L} \\
& =\int_{100}^{1150}\left\{f S L\left(T_{S L}\right) \int_{\left(10+2 T_{S L}\right) / 2.1}^{\infty} f W L\left(T_{W L}\right) \mathrm{d} T_{W L}\right\} \mathrm{d} T_{S L} \\
& =\int_{500}^{1000}\left\{(1 / 500) \int_{\left(10+2 T_{S L}\right) / 2.1}^{\infty} f W L\left(T_{W L}\right) \mathrm{d} T_{W L}\right\} \mathrm{d} T_{S L} \\
& =(1 / 500) \int_{500}^{F^{-1}(600)}\left\{\int_{\left(10+2 T_{S L}\right) / 2.1}^{600}(1 / 400) \mathrm{d} T_{W L}\right\} \mathrm{d} T_{S L} \\
& =\left(1 / 2 \times 10^{5}\right) \int_{500}^{625}\left\{\int_{\left(10+2 T_{S L}\right) / 2.1}^{600} 1 \mathrm{~d} T_{W L}\right\} \mathrm{d} T_{S L} \\
& \vdots \\
& =0.0372
\end{aligned}
$$

with the omitted steps again involving elementary calculus manipulations. Thus, as should be the case, integration on time with the representation for $p F$ in Eq. (2.12) and integration on temperature with the representation for $p F$ in Eq. (2.17) produce the same failure probabilities.

\subsection{Numerical Evaluation: Quadrature}

The preceding section (Sect. 2.2) provides a simple example in which it is possible to analytically carry out the integrations used in the definition of the failure probability $p F$. In practice, few problems are likely to be encountered in which it is possible to determine $p F$ in this manner. Rather, some type of numerical approximation will be required. This section considers approximations based on numerical integration (i.e., quadrature). Only integrals over temperature as in Eq. (2.17) will be considered; however, the same general ideas are applicable to integrals over time of the form in Eq. (2.12). 
The defining integral for $p F$ in Eq. (2.17) can be viewed as being of the form

$$
p F=\int_{T M N S L}^{T M X S L} G\left(T_{S L}\right) \mathrm{d} T_{S L}
$$

where

$$
\begin{aligned}
G\left(T_{S L}\right) & =f S L\left(T_{S L}\right) \int_{F\left(T_{S L}\right)}^{\infty} f W L\left(T_{W L}\right) \mathrm{d} T_{W L} \\
& =f S L\left(T_{S L}\right) I\left[F\left(T_{S L}\right), \infty, f W L\right] .
\end{aligned}
$$

Thus, if $G\left(T_{S L}\right)$ can be defined or approximated in a reasonably simple manner, then the evaluation of $p F$ only involves the approximation of an integral of a single variable. In particular, the goal is to design the analysis so that the integral designated by $\left.I F\left(T_{S L}\right), \infty, f W L\right]$ does not require a complex numerical evaluation. Because $I F\left(T_{S L}\right), \infty$, fWL] involves the integral of a probability density function (i.e., $f W L$ ), it is indeed possible to do this.

Possibilities for the density functions $f S L$ and $f W L$ include correspondence to uniform distributions, loguniform distributions, normal distributions, and lognormal distributions. The density functions for such distributions are given by

$$
f_{u}(T)= \begin{cases}1 /(b-a) & \text { for } a \leq T \leq b \\ 0 & \text { otherwise }\end{cases}
$$

for $T$ uniform on $[a, b]$,

$$
f_{l u}(T)= \begin{cases}1 /[T \ln (b / a)] & \text { for } a \leq T \leq b \\ 0 & \text { otherwise }\end{cases}
$$

for $T$ loguniform on $[a, b]$ with $a>0$,

$$
f_{n}(T)=(1 / \sigma \sqrt{2 \pi}) \exp \left[-(T-\mu)^{2} / 2 \sigma^{2}\right]
$$

for $T$ normal with mean $\mu$ and standard deviation $\sigma$, and

$$
f_{\ln }(T)=(1 / \sigma T \sqrt{2 \pi}) \exp \left[-(\ln T-\mu)^{2} / 2 \sigma^{2}\right]
$$

for $T$ lognormal with $T>0, E(\ln T)=\mu, V(\ln T)=\sigma^{2}$, and $E$ and $V$ used to represent expected value and variance, respectively. For the lognormal distribution, the expected value and variance of $T$ are given by

$$
E(T)=\exp \left(\mu+\sigma^{2} / 2\right)
$$


and

$$
V(T)=\left[\exp \left(\sigma^{2}\right)-1\right] \exp \left(2 \mu+\sigma^{2}\right)
$$

where $\mu$ and $\sigma^{2}$ are the corresponding mean and variance for $\ln T$ appearing in Eq. (2.33).

Determination of $G\left(T_{S L}\right)$ in Eq. (2.28) requires evaluation of the integral represented by $I\left[F\left(T_{S L}\right), \infty, f W L\right]$, which equals the probability of having a WL failure temperature that exceeds $F\left(T_{S L}\right)$ and thus, in effect, defines the CCDF associated with the distribution defined by the density function $f W L$. Significant computational savings can be achieved by precalculating the functional form of this integral, and then reusing this functional form rather than repeatedly evaluating the underlying integral. Specifically,

$$
\begin{aligned}
I\left[c, \infty, f_{u}\right] & =\int_{c}^{\infty} f_{u}(T) \mathrm{d} T \\
& = \begin{cases}1 & \text { if } c \leq a \\
(b-c) /(b-a) & \text { if } \mathrm{a} \leq c \leq b \\
0 & \text { if } \mathrm{b} \leq c\end{cases}
\end{aligned}
$$

for $-\infty<c<\infty$,

$$
\begin{aligned}
I\left[c, \infty, f_{l u}\right] & =\int_{c}^{\infty} f_{l u}(T) \mathrm{d} T \\
& = \begin{cases}1 & \text { if } c \leq a \\
\ln (b / c) / \ln (b / a) & \text { if } \mathrm{a} \leq c \leq b \\
0 & \text { if } \mathrm{b} \leq c\end{cases}
\end{aligned}
$$

for $-\infty<c<\infty$,

$$
\begin{aligned}
I\left[c, \infty, f_{n}\right] & =\int_{c}^{\infty} f_{n}(T) \mathrm{d} T \\
& =\int_{c}^{\infty}(1 / \sigma \sqrt{2 \pi}) \exp \left[-(T-\mu)^{2} / 2 \sigma^{2}\right] \mathrm{d} T
\end{aligned}
$$

for $-\infty<c<\infty$, and

$$
\begin{aligned}
I\left[c, \infty, f_{l n}\right] & =\int_{c}^{\infty} f_{\ln }(T) \mathrm{d} T \\
& =\int_{c}^{\infty}(1 / \sigma T \sqrt{2 \pi}) \exp \left[-(\ln T-\mu)^{2} / 2 \sigma^{2}\right] \mathrm{d} T \\
& =\int_{\ln c}^{\infty}(1 / \sigma \sqrt{2 \pi}) \exp \left[-(y-\mu)^{2} / 2 \sigma^{2}\right] \mathrm{d} y
\end{aligned}
$$

for $0<c<\infty$. 
Closed form representations for the integrals in Eqs. (2.38) and (2.39) do not exist. However, owing to the wide occurrence of such integrals, extensive effort has been devoted to developing compact approximations. For example,

$$
I\left[c, \infty, f_{n}\right]=1-P[(c-\mu) / \sigma]
$$

for $-\infty<c<\infty$ and

$$
I\left[c, \infty, f_{\ln }\right]=1-P[(\ln c-\mu) / \sigma]
$$

for $0<\mathrm{c}<\infty$, where $P(x)$ denotes the Gaussian probability integral defined by

$$
\begin{aligned}
P(x) & =(1 / \sqrt{2 \pi}) \int_{-\infty}^{x} \exp \left(-t^{2} / 2\right) \mathrm{d} t \\
& =1-(1 / \sqrt{2 \pi}) \int_{x}^{\infty} \exp \left(-t^{2} / 2\right) \mathrm{d} t .
\end{aligned}
$$

Specifically, Eq. (2.40) is obtained from Eq. (2.38) and the change of variables formula in Eq. (2.16) with $g(c)=(c$ $-\mu) / \sigma$, and Eq. (2.41) is obtained similarly from Eq. (2.39) with $g(c)=(\ln c-\mu) / \sigma$. In turn, $P(x)$ can be approximated by

$$
\begin{aligned}
P(x) \cong & 1-(1 / \sqrt{2 \pi}) \exp \left(-x^{2} / 2\right) t(\{[(1.330274429 t-1.821255978) t+1.781477937] t \\
& -0.356563782\} t+0.31938153)+\varepsilon(x),
\end{aligned}
$$

where $t=(1+0.2316419 x)^{-1}$ and $|\varepsilon(x)| \leq 7.5 \times 10^{-8}$ (see Sect. 16.3, Ref. [18]).

Another approximation is possible based on the relationships

$$
I\left[c, \infty, f_{n}\right]=(1 / 2) \operatorname{erf} c[(c-\mu) / \sigma \sqrt{2}]
$$

for $-\infty<c<\infty$ and

$$
I\left[c, \infty, f_{l n}\right]=(1 / 2) \operatorname{erfc}[(\ln c-\mu) / \sigma \sqrt{2}]
$$

for $o<\mathrm{c}<\infty$, where $\operatorname{erfc}(x)$ denotes the complementary error function defined by

$$
\operatorname{erfc}(x)=(2 / \sqrt{\pi}) \int_{x}^{\infty} \exp \left(-t^{2}\right) \mathrm{d} t
$$

In turn, $\operatorname{erfc}(x)$ can be approximated by

$$
\operatorname{erfc}(x) \cong t \exp \left(-x^{2}-1.26551223+t(1.00002368+t(0.37409196+t(0.09678418+t(-0.18628806\right.
$$




$$
+t(0.27886807+t(-1.13520398+t(1.48851587)+t(-0.82215223+t 0.170872777))))))))))
$$

for $x \geq 0$ and $t=1 /(1+0.5 x)$ and by

$$
\operatorname{erfc}(x)=2-\operatorname{erfc}(|x|)
$$

for $x<0$ (p. 164, Ref. [19]). The preceding approximation has a fractional (i.e., relative) error everywhere less than $1.2 \times 10^{-7}$.

Thus, $G\left(T_{S L}\right)$ can be approximated with expressions that are relatively easy to evaluate (i.e., numerically, not by "hand") for uniform, loguniform, normal and lognormal distributions. As a result, evaluation of $p F$ is really just a problem in the numerical integration of a function of a single variable. The numerical evaluation of such integrals is a rich and well-studied field and many techniques are available (e.g., Refs [20, 21]). The extended trapezoidal rule provides a simple but often adequate procedure for the numerical evaluation of integrals (pp. $107-115$, Ref. [19]). With this procedure, the integral in Eq. (2.28) defining $p F$ is approximated by

$$
\int_{T M N S L}^{T M X S L} G\left(T_{S L}\right) \mathrm{d} T_{S L} \cong h\left[G_{1} / 2+\sum_{i=2}^{N-1} G_{i}+G_{N} / 2\right]+O\left(1 / N^{2}\right),
$$

where $h=(T M X S L-T M N S L) /(N-1), T_{i}=T M N S L+h(i-1)$ for $i=1,2, \ldots, N, G_{i}=G\left(T_{i}\right)$ for $i=1,2, \ldots, N$, and the term $O\left(1 / N^{2}\right)$ indicates that there exists a constant $K$ such that the error in the approximation is bounded by $K\left(1 / N^{2}\right)=K / N^{2}$ for all sufficiently large values of $N$. A higher order approximation (i.e., $O\left(1 / N^{4}\right)$ rather than $\left.O\left(1 / N^{2}\right)\right)$ is provided by the extended Simpsons rule (p. 108, Ref. 19) With this procedure,

$$
\int_{T M N S L}^{T M X S L} G\left(T_{S L}\right) \mathrm{d} T_{S L} \cong h\left[G_{1}+4 G_{2}+2 G_{3}+4 G_{4}+\cdots+2 G_{N-2}+4 G_{N-1}+G_{N}\right] / 3+O\left(1 / N^{4}\right)
$$

where the 4,2 alteration continues throughout the interior of the summation and all other notation is the same as in Eq. (2.49). However, higher order does not necessarily imply higher accuracy. If neither of the preceding procedures is adequate, more sophisticated procedures are available. ${ }^{20,21}$

A relative error tolerance criterion can be used to terminate the approximation procedures indicated in Eqs. (2.49) and (2.50). Specifically, a relative error tolerance $\varepsilon>0$ is specified and the integration process is continued with increasing values of $N$ obtained halfing $h$ at each step (i.e., $N=3,5,9,17, \ldots$ ). The approximation process is terminated when the absolute value of the difference between the last two approximations (i.e., the previous approximation and the current approximation) is less than the product of the relative error tolerance $\varepsilon$ and the absolute value of the previous approximation. All quadrature results in this presentation are calculated with a relative error tolerance of $\varepsilon=10^{-5}$. 


\subsection{Numerical Evaluation: Monte Carlo}

A Monte Carlo procedure for the evaluation of the failure probability $p F$ is now presented. ${ }^{21,22}$ For this procedure, $p F$ is represented by

$$
p F=\int_{-\infty}^{\infty} \int_{-\infty}^{\infty} \delta\left(T_{W L}, T_{S L}\right) f W L\left(T_{W L}\right) f S L\left(T_{S L}\right) \mathrm{d} T_{W L} \mathrm{~d} T_{S L}
$$

where integrals from $-\infty$ to $\infty$ are used for notational convenience, the indicator function $\delta\left(T_{W L}, T_{S L}\right)$ is defined by

$$
\begin{array}{rlrl}
\delta\left(T_{W L}, T_{S L}\right)=1 & & \text { if } T_{S L}<T M P S L(t M I N) \text { and either } T M P W L(t M I N) \leq T_{W L} \text { or } \\
& T_{S L}<T_{W L}<T M P W L(t M I N) \\
=1 & & \text { if } \operatorname{TMPSL}(t M I N) \leq T_{S L} \leq \operatorname{TMPSL}(t M A X) \\
& & \text { and } \operatorname{TMPSL}^{-1}\left(T_{S L}\right)<\operatorname{TMPWL}^{-1}\left(T_{W L}\right) \\
=0 & & \text { otherwise }
\end{array}
$$

and the notational convention

$$
T M P W L^{-1}\left(T_{W L}\right)=\infty \text { if } T_{W L}>\operatorname{TMPWL}(t M A X)
$$

is adopted for use in Eq. (2.53).

The role of the indicator function $\delta\left(T_{W L}, T_{S L}\right)$ defined in Eqs. (2.52) - (2.54) is to "pick out" the failure temperature pairs $\left(T_{W L}, T_{S L}\right)$ in which the SL fails before the $W L$. In particular, (i) the assignment in Eq. (2.52) picks out the pairs in which the SL fails before the start of the analysis at time $t M I N$ and, for the special case with the WL also failing before $t M I N$, in which $T_{S L}$ is less than $T_{W L}$; (ii) the assignment in Eq. (2.53) picks out the pairs in which the SL fails between $t M I N$ and $t M A X$ before the failure of the $W L$; and (iii) the assignment in Eq. (2.54) removes the probability associated with pairs in which the SL does not fail before the WL from incorporation into $p F$. In practice, the limits of integration in Eq. (2.51) are determined by the density functions $f W L\left(T_{W L}\right)$ and $f S L\left(T_{S L}\right)$.

The integral defining $p F$ in Eq. (2.51) can be approximated by

$$
p F \cong \sum_{i=1}^{N} \delta\left(T_{W L, i}, T_{S L, i}\right) / N,
$$

where

$$
\left[T_{W L, i}, T_{S L, i}\right], i=1,2, \ldots, N
$$

is a random sample from the possible values of $T_{W L}$ and $T_{S L}$ generated in consistency with the distributions defined by the density functions $f S L\left(T_{W L}\right)$ and $f S L\left(T_{S L}\right)$. The preceding approximation will converge to the same value of 
$p F$ as the approximations in Eqs. (2.49) and (2.50). However, the convergence will be slower as the order of the Monte Carlo approximation is $O(1 / \sqrt{N})$ and the orders of the approximations in Eqs. (2.49) and (2.50) are $O\left(1 / N^{2}\right)$ and $O\left(1 / N^{4}\right)$, respectively.

Probably the greatest value of the Monte Carlo approximation to $p F$ is that it provides an independent means of verifying the complex, but more numerically efficient, approximation procedures presented in Sect. 2.3. In particular, procedures of the form described in Sect. 2.3 involving normal and lognormal distributions are essentially impossible to check by hand. However, the Monte Carlo procedure provides an independent way to calculate $p F$ and thus verify that the procedures of Sect. 2.3 are operating correctly.

The Monte Carlo approximation of the integral in Eq. (2.51) is very inefficient when $p F$ is small. For example, approximately 1 out of every $10^{6}$ sample elements in Eq. (2.57) produces a nonzero result when $p F$ has a value in the vicinity of $10^{-6}$. Thus, a very large sample would be required to produce a reasonably converged estimate to $p F$. In such situations, the efficiency of the Monte Carlo approximation can be increased by using an importance sampling procedure ${ }^{23-29}$ that emphasizes subregions of the $\left[T_{W L}, T_{S L}\right]$ space in which the failure of the SL before the WL is known to be likely (i.e., regions that have large values of $T_{W L}$ and small values of $T_{S L}$ ).

With an importance sampling procedure, the integral in Eq. (2.51) defining $p F$ is reformulated as

$$
p F=\int_{-\infty}^{\infty} \int_{-\infty}^{\infty}\left[\frac{\delta\left(T_{W L}, T_{S L}\right) f W L\left(T_{W L}\right) f S L\left(T_{S L}\right)}{f I W L\left(T_{W L}\right) f I S L\left(T_{S L}\right)}\right] f I W L\left(T_{W L}\right) f I S L\left(T_{S L}\right) \mathrm{d} T_{W L} \mathrm{~d} T_{S L}
$$

where $f T W L\left(T_{W L}\right)$ and $f I S L\left(T_{S L}\right)$ are the density functions that correspond to the distributions used for importance sampling on $T_{W L}$ and $T_{S L}$, respectively. For example, $f I W L\left(T_{W L}\right)$ and $f I S L\left(T_{S L}\right)$ could be defined to emphasize large and small values of $T_{W L}$ and $T_{S L}$, respectively. The resulting approximation to $p F$ is given by

$$
p F \cong \sum_{i=1}^{N}\left[\frac{\delta\left(T_{W L, i}, T_{S L, i}\right) f W L\left(T_{W L, i}\right) f S L\left(T_{S L, i}\right)}{f I W L\left(T_{W L, i}\right) f I S L\left(T_{S L, i}\right)}\right] / N
$$

where $\left[T_{W L, i}, T_{S L, i}\right], i=1,2, \ldots, N$, is now a random sample from the possible values of $T_{W L}$ and $T_{S L}$ generated in consistency with the distributions defined by the density functions $f I W L\left(T_{W L}\right)$ and $f I S L\left(T_{S L}\right)$.

The efficacy of importance sampling depends on the appropriate selection of the sampling distributions defined by $f I W L\left(T_{W L}\right)$ and $f I S L\left(T_{S L}\right)$. A good choice for these distributions can accelerate a Monte Carlo estimate of $p F$ relative to simple random sampling. However, a poor choice for the sampling distributions can result in a slower convergence to $p F$ than would be obtained with random sampling. 
A single example of the use of importance sampling follows. For this example, it is assumed that the non-zero ranges for $T_{W L}$ and $T_{S L}$ are given by $\left[T W L_{l}, T W L_{u}\right]$ and $\left[T S L_{l}, T S L_{u}\right]$, respectively, which results in representation for $p F$ in Eq. (2.51) having the form

$$
p F=\int_{T S L_{l}}^{T S L_{u}} \int_{T W L_{l}}^{T W L_{u}} \delta\left(T_{W L}, T_{S L}\right) f W L\left(T_{W L}\right) f S L\left(T_{S L}\right) \mathrm{d} T_{W L} \mathrm{~d} T_{S L}
$$

Further, the importance sampling distributions for $T_{W L}$ and $T_{S L}$ on $\left[T W L_{l}, T W L_{u}\right]$ and $\left[T S L_{l}, T S L_{u}\right]$ are right triangular and left triangular, respectively, which results in more emphasis on large values of $T_{W L}$ and small values of $T_{S L}$ than is likely to be the case for the original definitions of $f W L\left(T_{W L}\right)$ and $f S L\left(T_{S L}\right)$ (e.g., if these distributions correspond to truncated normal distributions with ranges extending for many standard deviations on either side of their means). The resultant definitions for $f I W L\left(T_{W L}\right)$ and $f I S L\left(T_{S L}\right)$ are

$$
f I W L\left(T_{W L}\right)=2\left(T_{W L}-T W L_{l}\right) /\left(T W L_{u}-T W L_{l}\right)^{2}
$$

and

$$
f I S L\left(T_{S L}\right)=2\left(T S L_{u}-T_{S L}\right) /\left(T S L_{u}-T S L_{l}\right)^{2}
$$

In turn, the importance sampling approximation to $p F$ is

$$
\begin{aligned}
p F & \cong \sum_{i=1}^{N}\left[\frac{\delta\left(T_{W L, i}, T_{S L, i}\right) f W L\left(T_{W L, i}\right) f S L\left(T_{S L, i}\right)}{f I W L\left(T_{W L, i}\right) f I S L\left(T_{S L, i}\right)}\right] / N \\
& =\frac{\left(T W L_{u}-T W L_{l}\right)^{2}\left(T S L_{u}-T S L_{l}\right)^{2}}{4 N} \sum_{i=1}^{N}\left[\frac{\delta\left(T_{W L, i}, T_{S L, i}\right) f W L\left(T_{W L, i}\right) f S L\left(T_{S L, i}\right)}{\left(T_{W L, i}-T W L_{l}\right)\left(T S L_{u}-T_{S L, i}\right)}\right],
\end{aligned}
$$

where $\left[T_{W L, i}, T_{S L, i}\right], i=1,2, \ldots, N$, is sampled from $\left[T W L_{l}, T W L_{u}\right]$ and $\left[T S L_{l}, T S L_{u}\right]$ in consistency with the definitions of $f I W L\left(T_{W L}\right)$ and $f I S L\left(T_{S L}\right)$ in Eqs. (2.61) and (2.62).

\subsection{Numerical Evaluation: Simple Example}

The example of Sect. 2.2 is used to illustrate the numerical evaluation of the failure probability $p F$ with both quadrature and Monte Carlo procedures. For the quadrature procedures, $p F$ is given by

$$
\begin{aligned}
p F & =\int_{T M N S L}^{T M X S L} G\left(T_{S L}\right) \mathrm{d} T_{S L} \\
& =\int_{100}^{1150} f S L\left(T_{S L}\right) I\left[F\left(T_{S L}\right), \infty, f W L\right] \mathrm{d} T_{S L}
\end{aligned}
$$


as indicated in Eqs. (2.28) and (2.29), with $f W L\left(T_{W L}\right), f S L\left(T_{S L}\right), F\left(T_{S L}\right)$ and $\int\left[\mathrm{F}\left(T_{S L}\right), \infty, f W L\right]$ defined in Eqs. (2.22), (2.23), (2.24) and (2.36), respectively. For the Monte Carlo procedures $p F$ is given by

$$
\begin{aligned}
p F & =\int_{-\infty}^{\infty} \int_{-\infty}^{\infty} \delta\left(T_{W L}, T_{S L}\right) f W L\left(T_{W L}\right) f S L\left(T_{S L}\right) \mathrm{d} T_{W L} \mathrm{~d} T_{S L} \\
& =\int_{500}^{1000} \int_{200}^{600} \delta\left(T_{W L}, T_{S L}\right) f W L\left(T_{W L}\right) f S L\left(T_{S L}\right) \mathrm{d} T_{W L} \mathrm{~d} T_{S L}
\end{aligned}
$$

as indicated in Eqs. (2.51) - (2.54), with $f W L\left(T_{W L}\right)$ and $f S L\left(T_{S L}\right)$ again defined in Eqs. (2.22) and (2.23).

The representation for $p F$ in Eq. (2.64) is evaluated with the trapezoidal rule (see Eq. (2.49)) and Simpson's rule (see Eq. (2.50)), and the representation for $p F$ in Eq. (2.65) is evaluated with simple random sampling (see Eq. (2.56)) and importance sampling based on right and left triangular distributions for $T_{W L}$ and $T_{S L}$, respectively (see Eqs. (2.60) - (2.63)). All approximations converge to values for $p F$ that are equal to, or very close to, the analytically calculated value of $p F=3.720 \times 10^{-2}$ (Table 2.1). Further, the convergence behavior of the approximations with the trapezoidal rule and Simpson's rule are similar, and the selected importance sampling procedure (i.e., right and left triangular for WL and WL failure temperature, respectively) shows little advantage over sampling directly from the assigned failure temperature distributions. Thus, the higher order Simpson's rule does not necessarily outperform the lower order trapezoidal rule, and the use of an intuitively posited importance sampling procedure does not necessarily outperform random sampling of the assigned failure temperature distributions. The numerical evaluation of $p F$ with the trapezoidal rule and Simpson's rule required significantly fewer function evaluations than the sampling-based evaluations (e.g., 16,385 versus 1,000,000). Further, the numerical results required more function evaluations than may actually be the case in many calculations due to the discontinuities at the ends of the uniform failure temperature distributions (e.g., see the sample results in Sects. 2.6, 3.4, 4.4 and 5.4).

\subsection{Numerical Evaluation: More Complex Example}

An example is presented in which the WL and SL temperature curves are nonlinear functions of time and the failure temperatures for the WL and SL are characterized by normal distributions. The WL and SL temperatures are defined by

$$
\begin{aligned}
& \operatorname{TMPWL}(t)=c_{1}+\left[c_{2}+c_{3} e^{-c_{4} t} \sin \left(c_{5} t\right)\right] \tanh \left(c_{6} t\right) \\
& \operatorname{TMPSL}(t)=c_{1}+c_{2} \tanh \left[c_{6}\left(1+c_{7}\right) t\right]
\end{aligned}
$$

where

$c_{1}=$ initial temperature $\left({ }^{\circ} \mathrm{C}\right)$ of the WL and SL $\left(10^{\circ} \mathrm{C}\right)$,

$c_{2}=$ increase in temperature $\left({ }^{\circ} \mathrm{C}\right)$ of the $\mathrm{WL}$ and $\mathrm{SL}$ at steady-state conditions $\left(900^{\circ} \mathrm{C}\right)$,

$c_{3}=$ peak amplitude $\left({ }^{\circ} \mathrm{C}\right)$ of a temperature transient of the $\mathrm{WL}\left(-1000{ }^{\circ} \mathrm{C}\right)$, 
Table 2.1. Approximations of Failure Probability $p F$ for System Defined in Sect. 2.2 with One WL, One SL and Uniform Distributions for WL and SL Failure Temperatures ${ }^{a}$

\begin{tabular}{|c|c|c|c|c|c|c|}
\hline & $\mathrm{N}^{b}$ & $\begin{array}{l}\text { Trapezoidal } \\
\text { Rule }^{\mathrm{c}}\end{array}$ & $\begin{array}{l}\text { Simpson's } \\
\text { Rule }^{\mathrm{d}}\end{array}$ & $\mathrm{N}^{\mathrm{e}}$ & $\begin{array}{l}\text { Random } \\
\text { Samplingf }\end{array}$ & $\begin{array}{l}\text { Importance } \\
\text { Samplingg }\end{array}$ \\
\hline & 3 & $0.000 \mathrm{E}+00$ & $0.000 \mathrm{E}+00$ & 1,000 & $3.300 \mathrm{E}-02$ & $3.998 \mathrm{E}-02$ \\
\hline & 5 & $0.000 \mathrm{E}+00$ & $0.000 \mathrm{E}+00$ & 10,000 & $3.950 \mathrm{E}-02$ & $3.737 \mathrm{E}-02$ \\
\hline & 9 & $0.000 \mathrm{E}+00$ & $0.000 \mathrm{E}+00$ & 100,000 & $3.781 \mathrm{E}-02$ & $3.700 \mathrm{E}-02$ \\
\hline & 17 & $2.051 \mathrm{E}-02$ & $2.734 \mathrm{E}-02$ & $1,000,000$ & $3.734 \mathrm{E}-02$ & $3.722 \mathrm{E}-02$ \\
\hline & 33 & $3.076 \mathrm{E}-02$ & $3.418 \mathrm{E}-02$ & $10,000,000$ & $3.722 \mathrm{E}-02$ & $3.719 \mathrm{E}-02$ \\
\hline & 65 & $3.589 \mathrm{E}-02$ & $3.760 \mathrm{E}-02$ & $100,000,000$ & $3.718 \mathrm{E}-02$ & $3.721 \mathrm{E}-02$ \\
\hline & 129 & $3.845 \mathrm{E}-02$ & $3.931 \mathrm{E}-02$ & & & \\
\hline & 257 & $3.725 \mathrm{E}-02$ & $3.685 \mathrm{E}-02$ & & & \\
\hline & 513 & $3.665 \mathrm{E}-02$ & $3.645 \mathrm{E}-02$ & & & \\
\hline & 1025 & $3.696 \mathrm{E}-02$ & $3.706 \mathrm{E}-02$ & & & \\
\hline & 2049 & $3.711 \mathrm{E}-02$ & $3.716 \mathrm{E}-02$ & & & \\
\hline & 4097 & $3.718 \mathrm{E}-02$ & $3.721 \mathrm{E}-02$ & & & \\
\hline & 8193 & $3.722 \mathrm{E}-02$ & $3.724 \mathrm{E}-02$ & & & \\
\hline & 16385 & $3.720 \mathrm{E}-02$ & $3.720 \mathrm{E}-02$ & & & \\
\hline & 32769 & $3.719 \mathrm{E}-02$ & $3.719 \mathrm{E}-02$ & & & \\
\hline & 65537 & $3.720 \mathrm{E}-02$ & $3.720 \mathrm{E}-02$ & & & \\
\hline & 131073 & $3.720 \mathrm{E}-02$ & $3.720 \mathrm{E}-02$ & & & \\
\hline & 262145 & $3.720 \mathrm{E}-02$ & $3.720 \mathrm{E}-02$ & & & \\
\hline & 524289 & $3.720 \mathrm{E}-02$ & $3.720 \mathrm{E}-02$ & & & \\
\hline \multicolumn{7}{|c|}{ a Calculations performed with CPLOAS program (App. III). } \\
\hline & \multicolumn{6}{|c|}{$\begin{array}{l}\text { Number of evaluations of } G\left(T_{S L}\right) \text { (see Eq. (2.29)) with trapezoidal rule and Simpson's rule, which corresponds to the interval [TMNSL, } \\
T M X S L] \text { being divided into } N-1 \text { subintervals and a relative error tolerance of } \varepsilon=10^{-5} \text {. }\end{array}$} \\
\hline $\mathrm{c}$ & \multicolumn{6}{|c|}{ Approximation to $p F$ obtained with trapezoidal rule (see Eq. (2.49)) and a relative error tolerance of $\varepsilon=10^{-5}$. } \\
\hline & \multicolumn{6}{|c|}{ Approximation to $p F$ obtained with Simpson's rule (see Eq. (2.50)) and a relative error tolerance of $\varepsilon=10^{-5}$. } \\
\hline e & \multicolumn{6}{|c|}{ Number of evaluation of $\delta\left(T_{W L}, T_{S L}\right)$ (see Eqs. (2.52) - (2.54)) for random sampling and importance sampling. } \\
\hline f & \multicolumn{6}{|c|}{ Approximation to $p F$ obtained with random sampling (see Eq. (2.56)). } \\
\hline $\mathrm{g}$ & \multicolumn{6}{|c|}{$\begin{array}{l}\text { Approximation to } p F \text { obtained with importance sampling with right and left triangular distributions for } T_{W L} \text { and } T_{S L} \text {, respectively (see Eqs. } \\
(2.58)-(2.63) \text { ). }\end{array}$} \\
\hline
\end{tabular}

$c_{4}=$ thermal heating time constant $\left(\mathrm{min}^{-1}\right)$ of a temperature transient of the WL $\left(0.30 \mathrm{~min}^{-1}\right)$,

$c_{5}=$ frequency of response $\left(\mathrm{min}^{-1}\right)$ of a temperature transient of the WL $\left(0.17 \mathrm{~min}^{-1}\right)$,

$c_{6}=$ time constant $\left(\mathrm{min}^{-1}\right)$ determining rate at which WL attains steady-state temperature $\left(0.03 \mathrm{~min}^{-1}\right)$,

$c_{7}=$ additive time constant (dimensionless) that accounts for more rapid heating in the SL than in the WL (0.6).

The values for $c_{1}, c_{2}, \ldots, c_{7}$ used in the following example are indicated in parentheses (see Sect. 4, Ref. [30]), and the resultant time-temperature curves are shown in Fig. 2.3. The time-temperature curves in Eqs. (2.66) and (2.67) 
and the associated definitions of $c_{1}, c_{2}, \ldots, c_{7}$ were selected to mimic the time-dependent behavior of results obtained in actual analyses of WL/SL systems (i.e., through a numerically demanding solution of a system of nonlinear partial differential equations). As used, quantities such as $c_{3}$ are not real physical properties of a system but rather devices employed in the emulation of results obtained with a mechanistic model.

Use of the representations in Eqs. (2.66) and (2.67) simplifies the presentation of the example but does not fundamentally deviate from the character of a real problem. In practice, the time-temperature results will be obtained as sequences of time-temperature pairs which would be smoothed or interpolated on in some way to obtain results corresponding to those in Eqs. (2.66) and (2.67) for use in determination of the failure probability $p F$. The quantities $c_{1}, c_{2}, \ldots, c_{7}$ will be used later in this presentation in the illustration of uncertainty and sensitivity analysis procedures applied to $p F$ (see Sect. 6).

The density functions for the normal distributions (see Eq. (2.32)) characterizing the WL and SL failure temperatures are given by

$$
\begin{aligned}
& f W L\left(T_{W L}\right)=\left(1 / c_{9} \sqrt{2 \pi}\right) \exp \left[-\left(T_{W L}-c_{8}\right)^{2} / 2 c_{9}^{2}\right] \\
& f S L\left(T_{S L}\right)=\left(1 / c_{11} \sqrt{2 \pi}\right) \exp \left[-\left(T_{S L}-c_{10}\right)^{2} / 2 c_{11}^{2}\right],
\end{aligned}
$$

where $c_{8}$ and $c_{9}$ are the mean and standard deviation for the WL failure temperature distribution and $c_{10}$ and $c_{11}$ are defined similarly for the SL failure temperature distribution. For the example, $c_{8}=310^{\circ} \mathrm{C}, c_{9}=8^{\circ} \mathrm{C}, c_{10}=560^{\circ} \mathrm{C}$ and $c_{11}=18^{\circ} \mathrm{C}$ (see Sect. 4, Ref. [30]). As for $c_{1}, c_{2}, \ldots, c_{7}$, the quantities $c_{8}, c_{9}, c_{10}, c_{11}$ will be used at a later point in the presentation in the illustration of uncertainty and sensitivity analysis procedures.

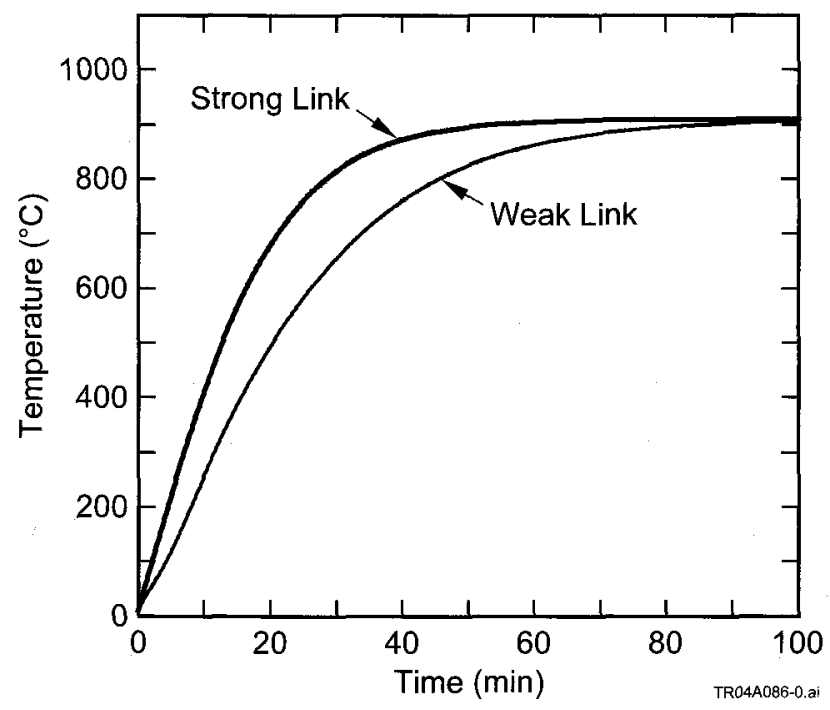

Fig. 2.3. Time-temperature curves defined in Eqs. (2.66) and (2.67) and used to illustrate calculation of failure probability $p F$. 
Similarly to the example in Sect. 2.5 , the failure probability $p F$ can be calculated with the trapezoidal rule and Simpson's rule from the representation in Eq. (2.64), except that now $T M P W L(t), \operatorname{TMPSL}(t), f W L\left(T_{W L}\right)$ and $f S L\left(T_{S L}\right)$ are defined in Eqs. (2.66) - (2.69). The function $F\left(T_{S L}\right)$ is defined in conjunction with Eq. (2.15), and $I\left[F\left(T_{S L}\right), \infty, f W L\right)$ is defined by the approximation obtained by combining Eqs. (2.44), (2.47) and (2.48). Results obtained with the trapezoidal rule and Simpson's rule are given in Table 2.2.

The failure probability $p F$ can also be calculated with Monte Carlo procedures used in conjunction with the representation in Eq. (2.65). Both simple random sampling and importance sampling can be used. For this example, importance sampling is illustrated with uniform sampling on the failure temperatures, which is equivalent to sampling uniformly with respect to standard deviation (i.e., if the temperature intervals $[a, b]$ and $[c, d]$ for one of the failure temperatures are both of length $k \sigma$, where $\sigma$ is the corresponding standard deviation for that failure temperature, then both intervals will contain approximately the same number of sampled temperatures). For calculation, the importance sampling distributions are defined over the intervals $\mu \pm 15 \sigma$ for each failure temperature distribution, which corresponds to $\left[150,390^{\circ} \mathrm{C}\right]$ and $\left[322.5,877.5^{\circ} \mathrm{C}\right]$ for the $\mathrm{WL}$ and the SL, respectively. As a result,

$$
f I W L\left(T_{W L}\right)=1 /\left(390^{\circ} \mathrm{C}-150^{\circ} \mathrm{C}\right)=4.167 \times 10^{-3}{ }^{\circ} \mathrm{C}^{-1}
$$

Table 2.2. Approximations of Failure Probability pF for System Defined in Section 2.6 with One WL, One SL and Normal Distributions for WL and SL Failure Temperatures ${ }^{a}$

\begin{tabular}{|c|c|c|c|c|c|c|}
\hline & $\mathrm{N}^{\mathrm{b}}$ & $\begin{array}{l}\text { Trapezoidal } \\
\text { Rule }\end{array}$ & $\begin{array}{l}\text { Simpson's } \\
\text { Ruled }\end{array}$ & $\mathrm{N}^{\mathrm{e}}$ & $\begin{array}{c}\text { Random } \\
\text { Sampling }\end{array}$ & $\begin{array}{l}\text { Importance } \\
\text { Samplingg }\end{array}$ \\
\hline & 3 & $1.831 \mathrm{E}-06$ & $2.441 \mathrm{E}-06$ & 1,000 & $0.000 \mathrm{E}+00$ & $3.386 \mathrm{E}-05$ \\
\hline & 5 & $9.155 \mathrm{E}-07$ & $6.103 \mathrm{E}-07$ & 10,000 & $0.000 \mathrm{E}+00$ & $1.744 \mathrm{E}-05$ \\
\hline & 9 & $4.577 \mathrm{E}-07$ & $3.052 \mathrm{E}-07$ & 100,000 & $4.000 \mathrm{E}-05$ & $1.511 \mathrm{E}-05$ \\
\hline & 17 & $6.895 \mathrm{E}-07$ & $7.667 \mathrm{E}-07$ & $1,000,000$ & $1.900 \mathrm{E}-05$ & $1.474 \mathrm{E}-05$ \\
\hline & 33 & $1.865 \mathrm{E}-05$ & $2.464 \mathrm{E}-05$ & $10,000,000$ & $1.540 \mathrm{E}-05$ & $1.499 \mathrm{E}-05$ \\
\hline & 65 & $1.512 \mathrm{E}-05$ & $1.394 \mathrm{E}-05$ & $100,000,000$ & $1.562 \mathrm{E}-05$ & $1.511 \mathrm{E}-05$ \\
\hline & 129 & $1.512 \mathrm{E}-05$ & $1.512 \mathrm{E}-05$ & & & \\
\hline & 257 & $1.512 \mathrm{E}-05$ & $1.512 \mathrm{E}-05$ & & & \\
\hline & 513 & $1.512 \mathrm{E}-05$ & $1.512 \mathrm{E}-05$ & & & \\
\hline \multicolumn{7}{|c|}{ a Calculations performed with CPLOAS program (App. III). } \\
\hline \multicolumn{7}{|c|}{$\begin{array}{l}\text { Number of evaluations of } G\left(T_{S L}\right) \text { (see Eq. (2.29)) with trapezoidal rule and Simpson's rule, which corresponds to the interval [TMNSL, } \\
\text { TMXSL] being divided into } N-1 \text { subintervals. }\end{array}$} \\
\hline \multicolumn{7}{|c|}{ Approximation to $p F$ obtained with trapezoidal rule (see Eq. (2.49)) and a relative error tolerance of $\varepsilon=10^{-5}$} \\
\hline \multicolumn{7}{|c|}{ Approximation to $p F$ obtained with Simpson's rule (see Eq. (2.50)) and a relative error tolerance of $\varepsilon=10^{-5}$. } \\
\hline e & \multicolumn{6}{|c|}{ Number of evaluation of $\delta\left(T_{W L}, T_{S L}\right)$ (see Eqs. (2.52) - (2.54)) for random sampling and importance sampling. } \\
\hline f & \multicolumn{6}{|c|}{ Approximation to $p F$ obtained with random sampling (see Eq. (2.56)). } \\
\hline g & \multicolumn{6}{|c|}{$\begin{array}{l}\text { Approximation to } p F \text { obtained with importance sampling with right and left triangular distributions for } T_{W L} \text { and } T_{S L} \text {, respectively (see Eqs. } \\
(2.58)-(2.63)) \text {. }\end{array}$} \\
\hline
\end{tabular}




$$
f \operatorname{fSL}\left(T_{S L}\right)=1\left(877.5^{\circ} \mathrm{C}-322.5^{\circ} \mathrm{C}\right)=1.802 \times 10^{-3}{ }^{\circ} \mathrm{C}^{-1},
$$

and the resultant importance sampling approximation to $p F$ is given as indicated in Eq. (2.59). Results obtained with simple random sampling and importance sampling are given in Table 2.2 .

The results obtained with importance sampling converge more rapidly than the results obtained with random sampling. As the sample size increases, the sampling-based results approach the quadrature-based results. However, the quadrature-based results required far fewer function evaluations than the sampling-based results.

\subsection{Comparison with One WL, One SL Representation Developed by Bohn}

The competing temperature-dependent failure of a WL/SL system has been previously considered by Bohn (App. I). For one WL and one SL and with the notation used in his presentation, Bohn's representation for the probability $p F$ that the SL fails before the WL for the system described in Sect. 2.1 is

$$
p F=\int_{t M I N}^{t M A X} C D F_{S L 1}(t) P D F_{W L 1}(t) \mathrm{d} t
$$

where

$C D F_{S L 1}(t)=$ probability that SL fails before time $t$

$P D F_{W L 1}(t)=$ probability density function for time at which the WL fails (i.e., $\left.I t_{1}, t_{2}, P D F_{W L 1}\right]$ is the probability that the WL fails between time $t_{1}$ and time $t_{2}$ ),

and $[t M I N, t M A X]$ is the time interval over which the calculation is carried out. This is the representation given in Eq. (4) of Bohn's report (App. I) with (i) one WL and one SL, (ii) the time interval $[0, \infty]$ replaced by $[t M I N, t M A X]$ (note: in practice $t M A X$ is always finite because calculations will never be carried out for $t M A X=\infty$ in a real problem), and (iii) $t_{0}$ replaced by $t$ for notational convenience.

In the notation used in this presentation, the representation for $p F$ in Eq. (2.72) becomes

$$
\begin{gathered}
p F=\int_{t M I N}^{t M A X}\left(\int_{t M I N}^{t}\{f S L[T M P S L(\tau)]\}\{\mathrm{d} T M P S L(\tau) / \mathrm{d} \tau\} \mathrm{d} \tau\right) \\
\cdot(\{f W L[T M P W L(t)]\}\{\mathrm{d} T M P W L(t) / \mathrm{d} t\}) \mathrm{d} t,
\end{gathered}
$$

where

$$
\begin{aligned}
& C D F_{S L 1}(t)=\int_{t M I N}^{t}\{f S L[T M P S L(\tau)]\}\{\mathrm{d} T M P S L(\tau) / \mathrm{d} \tau\} \mathrm{d} \tau \\
& P D F_{W L 1}(t)=\{f W L[T M P W L(t)]\}\{\mathrm{d} T M P W L(t) / \mathrm{d} t\} .
\end{aligned}
$$


The corresponding representations for $p F$ given in Eqs. (2.12) and (2.15) of this presentation are

$$
\begin{aligned}
p F & =\int_{t M I N}^{t M A X}\{f S L[T M P S L(t)]\}\{\mathrm{d} T M P S L(t) / \mathrm{d} t\}\left\{\int_{T M P W L(t)}^{\infty} f W L\left(T_{W L}\right) \mathrm{d} T_{W L}\right\} \mathrm{d} t \\
& =\int_{T M N S L}^{T M X S L} \int_{F\left(T_{S L}\right)}^{\infty} f W L\left(T_{W L}\right) f S L\left(T_{S L}\right) \mathrm{d} T_{W L} \mathrm{~d} T_{S L},
\end{aligned}
$$

where $F\left(T_{S L}\right)=T M P W L\left[T M P S L^{-1}\left(T_{S L}\right)\right]$ expresses the WL temperature as a function of the SL temperature. An unstated but underlying assumption with respect to Eqs. $(2.72)-(2.75)$ is that the probability of either the WL or the SL failing at a time before $t M I N$ or at a temperature below TMNSL is either zero or negligibly small.

Although they appear to be different, the representation for $p F$ in Eq. (2.73) and the representations for $p F$ in Eqs. (2.74) and (2.75) are effectively the same. This equivalence can be seen by using suitable changes of variable (see Eq. (2.16)) to reformulate the representation for $p F$ in Eq. (2.73). Specifically, Eq. (2.73) can be rewritten as

$$
\begin{aligned}
p F & =\int_{t M I N}^{t M A X}\left(\int_{T M N S L}^{T M P S L}(t) f S L\left(T_{S L}\right) \mathrm{d} T_{S L}\right)(\{f W L[T M P W L(t)]\}\{\mathrm{d} T M P W L(t) / \mathrm{d} t\}) \mathrm{d} t \\
& =\int_{T M N W L}^{T M X W L}\left(\int_{T M N S L}^{F^{-1}\left(T_{W L}\right)} f S L\left(T_{S L}\right) \mathrm{d} T_{S L}\right) f W L\left(T_{W L}\right) \mathrm{d} T_{W L} \\
& =\int_{T M N W L}^{T M X W L} \int_{T M N S L}^{F^{-1}\left(T_{W L}\right)} f S L\left(T_{S L}\right) f W L\left(T_{W L}\right) \mathrm{d} T_{S L} \mathrm{~d} T_{W L}
\end{aligned}
$$

where (i) the first equality results from the change of variables $T_{S L}=\operatorname{TMPSL}(\tau)$ in the inner integral in Eq. (2.73), (ii) the second equality results from the change of variables $T_{W L}=T M P W L(t)$ in the outer integral in the first equality and the use of $F^{-1}\left(T_{W L}\right), T M N W L$, and $T M X W L$ to represent $T M P S L\left[T M P W L^{-1}\left(T_{W L}\right)\right], T M P W L(t M I N)$ and $T M P W L(t M A X)$, respectively and (iii) the third equality results from a rearrangement of the terms in the second equality.

The functions $F\left(T_{S L}\right)$ and $F^{-1}\left(T_{W L}\right)$ are defined by

$$
F\left(T_{S L}\right)=T M P W L\left[T M P S L^{-1}\left(T_{S L}\right)\right] \text { and } F^{-1}\left(T_{S L}\right)=T M P S L\left[T M P W L^{-1}\left(T_{W L}\right)\right]
$$

with (i) $F\left(T_{S L}\right)$ providing the WL temperature associated with a SL temperature of $T_{S L}$ and (ii) $F^{-1}\left(T_{W L}\right)$ providing the SL temperature associated with a WL temperature of $T_{W L}$. Thus, as anticipated by the notation selected for use, the functions $F\left(T_{S L}\right)$ and $F^{-1}\left(T_{W L}\right)$ are inverses of each other (Fig. 2.4). Because of this inverse relationship, the double integrals in Eqs. (2.75) and (2.76) are effectively over the same area. Specifically, both integrals are over the cross-hatched area in Fig. 2.4, with (i) the outer integral in Eq. (2.76) along the abscissa (i.e., the $T_{W L}$ axis) from TMPWL to TMXWL and the inner integral up to the curve $\left[T_{W L}, F^{-1}\left(T_{W L}\right)\right]$, and (ii) the outer integral in Eq. (2.75) along the ordinate (i.e., the $T_{S L}$ axis) from $T M N S L$ and $T M X S L$ and the inner integral starting at the curve $\left[F\left(T_{S L}\right)\right.$, 
$\left.T_{S L}\right]$, which is the same as the curve $\left[T_{W L}, F^{-1}\left(T_{W L}\right)\right]$, and moving to the right. In addition, the integral in Eq. (2.75) covers the area to the right of $T M X W L$ on the abscissa. Geometrically, this is an infinite area. However, if the probability of having a WL failure temperature greater than TMXWL is zero, then the part of the integral in Eq. (2.75) over this area will be zero and so the representations for $p F$ in Eqs. (2.75) and (2.76) will produce the same value. Similarly, as long as the probability of a WL failure temperature larger than TMXWL is small, the representations for $p F$ in Eqs. (2.75) and (2.76) will produce numerically similar results. In summary, the representations for $p F$ in Eqs. (2.72) - (2.73) (i.e., in the Bohn development) and in Eqs. (2.74) - (2.75) (i.e., in the development of this presentaiton) produce the same value for $p F$ when the probability of the WL failing at a temperature greater than $T M X W L=T M P W L(t M A X)$ is zero. However, if the probability of the WL failing at a temperature above $T M X W L$ is greater than zero, then use of the representations in Eqs. (2.72) - (2.73) results in an underestimate of $p F$.

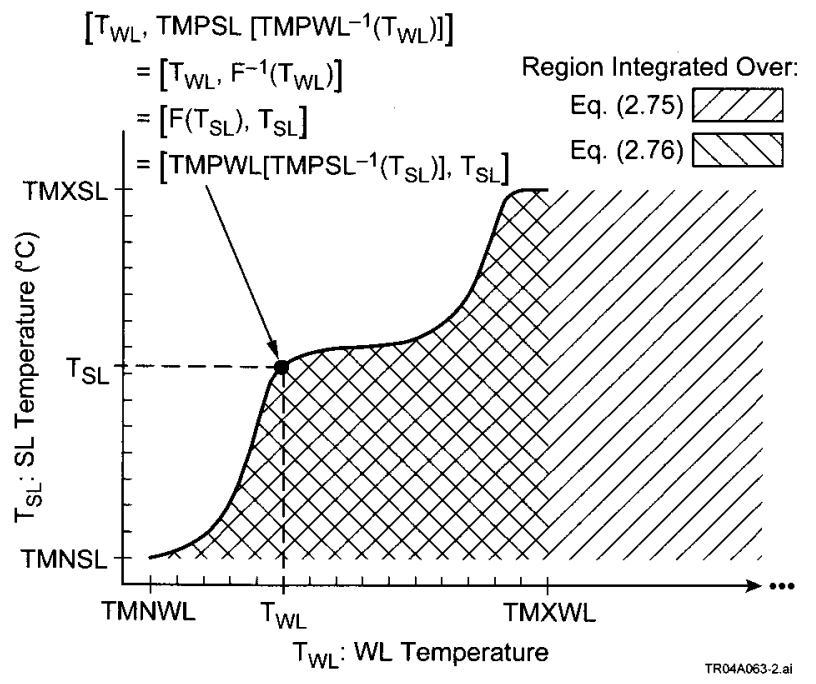

Fig. 2.4. Illustration of regions integrated over to determine the failure probability $p F$ with Eqs. (2.75) and (2.76) 


\section{Multiple WLs and SLs with Failure of One SL Before Any WL Constituting System Failure}

The analysis of PLOAS for a system involving multiple WLs and multiple SLs with failure of one SL before failure of any WL constituting system failure is now presented. The following topics are considered: formal mathematical representation of PLOAS (Sect. 3.1), numerical evaluation of defining integral for PLOAS with quadrature methods (Sect. 3.2), numerical evaluation of defining integral for PLOAS with Monte Carlo methods (Sect. 3.3), and an example of the numerical evaluation of PLOAS (Sect. 3.4).

\subsection{Formal Representation}

The system is now assumed to involve $n W L$ WLs and $n S L$ SLs. The system is assumed to fail if any SL fails before any of the WLs fail. The notation introduced in Sect. 2 is extended as follows:

$$
\begin{aligned}
T M P W L_{j}(t) & =\text { temperature }\left({ }^{\circ} \mathrm{C}\right) \text { of } \mathrm{WL} j, j=1,2, \ldots, n W L, \text { at time } t(\mathrm{~min}), \\
T M P S L_{k}(t) & =\text { temperature }\left({ }^{\circ} \mathrm{C}\right) \text { of } \mathrm{SL} k, k=1,2, \ldots, n S L, \text { at time } t(\min ), \\
f W L_{j} & =\text { density function }\left({ }^{\circ} \mathrm{C}^{-1}\right) \text { for failure temperature } T F W L_{j} \text { of } \mathrm{WL} j, j=1,2, \ldots, n W L, \\
f S L_{k} & =\text { density function }\left({ }^{\circ} \mathrm{C}^{-1}\right) \text { for failure temperature } T F S L_{k} \text { of SL } k, k=1,2, \ldots, n S L .
\end{aligned}
$$

The bounding times (i.e., $t M I N, t M A X$ ) remain as before; however, the individual SLs can have different minimum and maximum temperatures.

The objective of this section is to determine the probability $p F$ that a SL fails before any WL fails. The sample space underlying this calculation is

$$
\mathcal{S}=\left\{\mathbf{t}: \mathbf{t}=\left[t F S L_{1}, t F S L_{2}, \ldots, t F S L_{n S L}, t F W L_{1}, t F W L_{2}, \ldots, t F W L_{n S W}\right]\right\},
$$

where $t F S L_{k}$ and $t F W L_{j}$ are the failure times of the $k^{\text {th }} S L$ and $j^{\text {th }} \mathrm{WL}$, respectively. In particular,

$$
p F=\operatorname{prob}(\mathcal{E}),
$$

where $\mathcal{E}$ is the subset of $\mathcal{S}$ defined by

$$
\begin{gathered}
\varepsilon=\left\{\mathbf{t}: \mathbf{t}=\left[t F S L_{1}, t F S L_{2}, \ldots, t F S L_{n S L}, t F W L_{1}, t F W L_{2}, \ldots, t F W L_{n S W}\right] \in S \text { and there exists an integer } k\right. \\
\text { such that } \left.1 \leq k \leq n S L \text { and } t F S L_{k}<t F W L_{j} \text { for } j=1, \cdots 2, \ldots, n W L\right\} .
\end{gathered}
$$

Specifically, $\mathcal{E}$ contains the elements of $S$ for which a SL fails before any WL fails. 
The determination of $p F$ is based on the following decomposition of $\mathcal{E}$ :

$$
\varepsilon=\bigcup_{i=1}^{n T M}\left[\bigcup_{k=1}^{n S L}\left(\varepsilon_{i k} \cup \tilde{\varepsilon}_{i k}\right)\right]
$$

where

$$
\begin{aligned}
\mathcal{E}_{i k}= & \left\{\mathbf{t}: \mathbf{t} \in \mathcal{E}, t_{i-1} \leq t F S L_{k} \leq t_{i}<t F S L_{l} \text { for } l \neq k, t_{i}<t F W L_{j} \text { for } j=1,2, \ldots, n W L\right\}, \\
\tilde{\mathcal{E}}_{i k}= & \left\{\mathbf{t}: \mathbf{t} \in \mathcal{E}, t_{i-1} \leq t F S L_{k} \leq t_{i}, t F S L_{k} \leq t F S L_{l} \text { for } l \neq k, t F S L_{k} \leq t F W L_{j} \text { for } j=1,2, \ldots, n W L\right. \text { and either (i) there } \\
& \text { exists at least one value of } l \text { such that } t F S L_{l} \leq t_{i} \text { or (ii) there exists at least one value of } j \text { such that } t F W L_{j} \\
& \left.\leq t_{i}\right\},
\end{aligned}
$$

and $t M I N=t_{0}<t_{1}<\ldots<t_{n T M}=t M A X$ is a partition of $[t M I N, t M A X]$ as indicated in Fig. 2.1. The set $\tilde{\varepsilon}_{i k}$ contains elements of $\mathcal{E}$ that have a SL failure time $t F S L_{l}, l \neq k$, satisfying $t F S L_{k} \leq t F S L_{l} \leq t_{i}$ or a WL failure time $t F W L_{j}, j=$ $1,2, \ldots, n W L$, satisfying $t F S L_{k}<t F W L_{j} \leq t_{i}$. The equality

$$
\operatorname{prob}(\varepsilon)=\sum_{i=1}^{n T M} \sum_{k=1}^{n S L} \operatorname{prob}\left(\varepsilon_{i k}\right)+\sum_{i=1}^{n T M} \sum_{k=1}^{n S L} \operatorname{prob}\left(\tilde{\varepsilon}_{i k}\right)
$$

holds because the sets involved are disjoint (or, to be more precise, have intersections with a probability of zero, to cover the situation that arises for $\varepsilon_{i k}$ and $\varepsilon_{i l}$ when $t F S L_{k}=t F S L_{l}, 1 \leq k<l \leq n S L$ ).

The equality

$$
\lim _{n T M \rightarrow \infty} \sum_{i=1}^{n T M} \sum_{k=1}^{n S L} \operatorname{prob}\left(\tilde{\varepsilon}_{i k}\right)=0
$$

holds because the indicated probabilities involve products whose individual terms are proportional to $\Delta t_{i}$ (see Eq. (2.9) for an analogous situation). Further,

$$
\begin{aligned}
\operatorname{prob}\left(\varepsilon_{i k}\right)= & I\left[T M P S L_{k}\left(t_{i-1}\right), T M P S L_{k}\left(t_{i}\right), f S L_{k}\right] \prod_{\substack{l=1 \\
l \neq k}}^{n S L} I\left[T M P S L_{l}\left(t_{i}\right), \infty, f S L_{k}\right] \\
& \cdot \prod_{j=1}^{n W L} I\left[T M P W L_{j}\left(t_{i}\right), \infty, f W L_{j}\right] \\
\cong & \left\{\left[T M P S L_{k}\left(t_{i}\right)-\operatorname{TMPSL}_{k}\left(t_{i-1}\right)\right] f S L_{k}\left[\operatorname{TMPSL}_{k}\left(t_{i}\right)\right]\right\} \prod_{\substack{l=1 \\
l \neq k}}^{n S L} I\left[T M P S L_{l}\left(t_{i}\right), \infty, f S L_{l}\right] \\
& \cdot \prod_{j=1}^{n W L} I\left[T M P W L_{j}\left(t_{i}\right), \infty, f W L_{j}\right]
\end{aligned}
$$


where (i) $I[a, b, f]$ is defined in conjunction with Eqs. (2.9) and (2.10), (ii) the first term in the preceding products corresponds to the probability that SL $k$ fails in the time interval $\left[t_{i-1}, t_{i}\right]$, (iii) the second term corresponds to the probability that all SLs except SL $k$ fail after $t_{i}$, and (iv) the third term corresponds to the probability that all WLs fail after $t_{i}$.

As a result,

$$
\begin{aligned}
& \operatorname{prob}(\mathcal{E})= \lim _{n T M \rightarrow \infty} \sum_{i=1}^{n T M} \sum_{k=1}^{n S L} \operatorname{prob}\left(\varepsilon_{i k}\right)+\lim _{n T M \rightarrow \infty} \sum_{i=1}^{n T M} \sum_{k=1}^{n S L} \operatorname{prob}\left(\tilde{\varepsilon}_{i k}\right) \\
&= \lim _{n T M \rightarrow \infty} \sum_{i=1}^{n T M} \sum_{k=1}^{n S L} \operatorname{prob}\left(\varepsilon_{i k}\right)+0 \\
&= \lim _{n T M \rightarrow \infty} \sum_{i=1}^{n T M} \sum_{k=1}^{n S L}\left\{\left[T M P S L_{k}\left(t_{i}\right)-T M P S L_{k}\left(t_{i-1}\right)\right] f S L_{k}\left[T M P S L_{k}\left(t_{i}\right)\right]\right\} \\
& \cdot \prod_{l=1}^{n S L} I\left[T M P S L_{l}\left(t_{i}\right), \infty, f S L_{l}\right] \prod_{j=1}^{n W L} I\left[T M P W L_{j}\left(t_{i}\right), \infty, f W L_{j}\right]
\end{aligned}
$$

where the first, second and third equalities follow from Eqs. (3.9), (3.10) and (3.11), respectively. Evaluation of the final limit of Eq. (3.12) leads to the representation of $\operatorname{prob}(\mathcal{E})$, and hence $p F$, by

$$
\begin{aligned}
& p F=\sum_{k=1}^{n S L}\left(\int _ { t M I N } ^ { t M A X } \left\{f S L _ { k } [ T M P S L _ { k } ( t ) ] \left\{\left\{\prod_{\substack{l=1 \\
l \neq k}}^{n S L} I\left[T M P S L_{l}(t), \infty, f S L_{l}\right]\right\}\right.\right.\right. \\
& \left.\cdot\left\{\prod_{j=1}^{n W L} I\left[T M P W L_{j}(t), \infty, f W L_{j}\right]\right\} \mathrm{d} T M P S L_{k}(t)\right) \\
& =\int_{t M N}^{t M A X}\left(\sum_{k=1}^{n S L}\left\{f S L_{k}\left[T M P S L_{k}(t)\right]\right\}\left\{\prod_{\substack{l=1 \\
l \neq k}}^{n S L} I\left[T M P S L_{l}(t), \infty, f S L_{l}\right]\right\}\left\{\mathrm{d} T M P S L_{k}(t) / \mathrm{d} t\right\}\right) \\
& \cdot\left(\prod_{j=1}^{n W L} I\left[T M P W L_{j}(t), \infty, f W L_{j}\right]\right) \mathrm{d} t
\end{aligned}
$$

where the first integral is a Riemann-Stieltjes integral and the second integral is the corresponding Riemann integral.

The Riemann integral defining $p F$ in Eq. (3.13) is with respect to time. As discussed in conjunction with Eqs. (2.16) and (2.17), the definition of $p F$ can be converted to an integral with respect to temperature by a suitable change of variable. In particular, $p F$ can also be represented by 


$$
\begin{aligned}
& p F= \sum_{k=1}^{n S L}\left(\int_{T_{M N S L_{k}}}^{T M X S L_{k}}\left\{f S L_{k}\left(T_{S L}\right)\right\}\left\{\prod_{\substack{l=1 \\
l \neq k}}^{n S L} I\left[T M P S L_{l}\left[T M P S L_{k}^{-1}\left(T_{S L}\right)\right], \infty, f S L_{l}\right]\right\}\right. \\
&\left.\cdot\left\{\prod_{j=1}^{n W L} I\left[T M P W L_{j}\left[\operatorname{TMPSL}_{k}^{-1}\left(T_{S L}\right)\right], \infty, f W L_{j}\right]\right\}\right) \mathrm{d} T_{S L} \\
&=\int_{T M N S L}^{T M X S L} G\left(T_{S L}\right) \mathrm{d} T_{S L},
\end{aligned}
$$

where

$$
\begin{aligned}
T_{M N S L_{k}=} & T M P S L_{k}(t M I N), k=1,2, \ldots, n S L, \\
T M X S L_{k}= & T M P S L_{k}(t M A X), k=1,2, \ldots, n S L, \\
T M N S L= & \min \left\{T M N S L_{k}: k=1,2, \ldots, n S L\right\}, \\
T M X S L= & \max \left\{T M X S L_{k}: k=1,2, \ldots, n S L\right\}, \\
G\left(T_{S L}\right)= & \sum_{k=1}^{n S L} G_{k}\left(T_{S L}\right), \\
G_{k}\left(T_{S L}\right)= & f S L_{k}\left(T_{S L}\right)\left\{\prod_{l=1}^{n S L} I\left[T M P S L_{l}\left[T M P S L_{k}^{-1}\left(T_{S L}\right)\right], \infty, f S L_{l}\right]\right\} \\
l \neq k & \cdot\left\{\prod_{j=1}^{n W L} I\left[T M P W L_{j}\left[T M P S L_{k}^{-1}\left(T_{S L}\right)\right], \infty, f W L_{j}\right]\right\} \text { for } T M N S L_{k} \leq T_{S L} \leq T M X S L_{k} \\
= & 0 \text { otherwise, }
\end{aligned}
$$

and all integrals are now on temperature. The simpler representation

$$
\begin{aligned}
p F=\int_{T M N S L}^{T M X S L}\left(\sum_{k=1}^{n S L}\left\{f S L_{k}\left(T_{S L}\right)\right\}\left\{\prod_{\substack{l=1 \\
l \neq k}}^{n S L} I\left[T M P S L_{l}\left[T M P S L_{k}^{-1}\left(T_{S L}\right)\right], \infty, f S L_{l}\right]\right\}\right. \\
\left.\cdot\left\{\prod_{j=1}^{n W L} I\left[T M P W L_{j}\left[\operatorname{TMPSL}_{k}^{-1}\left(T_{S L}\right)\right], \infty, f W L_{j}\right]\right\}\right) \mathrm{d} T_{S L},
\end{aligned}
$$

for the first equality in Eq. (3.14) results when $T M N S L_{k}=T M N S L$ and $T M X S L_{k}=T M X S L$ for $k=1,2, \ldots, n S L$. 


\subsection{Numerical Evaluation: Quadrature}

The same numerical procedures that were described in Sect. 2.3 for one WL and one SL are also appropriate for the configuration described in the preceding section (Sect. 3.1) for $n W L$ WLs and $n S L$ SLs. In particular, as the representation for $p F$ in Eqs. (3.14) - (3.20) is the same as the representation described in Eqs. (2.28) and (2.29) except that the function $G\left(T_{S L}\right)$ is more complicated. However, the integrals in the definition of $G\left(T_{S L}\right)$ in Eqs. (3.19) and (3.20) can be approximated as described in Sect. 2.3. Thus, $G\left(T_{S L}\right)$ can be determined in an efficient manner, and as a result, $p F$ defined in Eqs. (3.14) - (3.20) can be evaluated with the integral approximations in Eqs. (2.49) and (2.50).

\subsection{Numerical Calculation: Monte Carlo}

Monte Carlo procedures similar to those described in Sect. 2.4 for one WL and one SL can also be used for the $\mathrm{WL} / \mathrm{SL}$ configuration described in Sect. 3.1. The determination of the failure probability $p F$ for the WL/SL configuration in Sect. 3.1 is based on the representation of $p F$ with the integral

$$
p F=\int_{-\infty}^{\infty} \ldots \int_{-\infty}^{\infty} \int_{-\infty}^{\infty} \ldots \int_{-\infty}^{\infty} \delta\left(\mathbf{T}_{W L}, \mathbf{T}_{S L}\right) \prod_{j=1}^{n W L} f W L_{j}\left(T_{W L, j}\right) \prod_{j=1}^{n S L} f S L_{k}\left(T_{S L, k}\right) \prod_{j=1}^{n W L} \mathrm{~d} T_{W L, j} \prod_{k=1}^{n S L} \mathrm{~d} T_{S L, k},
$$

where

$$
\begin{aligned}
& \mathbf{T}_{W L}=\left[T_{W L, 1}, T_{W L, 2}, \ldots, T_{W L, n W L}\right], \\
& \mathbf{T}_{S L}=\left[T_{S L, 1}, T_{S L, 2}, \ldots, T_{S L, n S L}\right], \\
& \delta\left(\mathbf{T}_{W L}, \mathbf{T}_{S L}\right)=\delta\left(T_{W L, 1}, T_{W L, 2}, \ldots, T_{W L, n W L}, T_{S L, 1}, T_{S L, 2}, \ldots, T_{S L, n S L}\right) \\
& =1 \text { if } S_{S L}\left(\mathbf{T}_{S L}\right) \neq \phi \text { and either (i) } S_{W L}\left(\mathbf{T}_{W L}\right)=\phi \text { or (ii) } S_{W L}\left(\mathbf{T}_{W L}\right) \neq \phi \text { and } \\
& \min \left\{T_{S L, j}: j \in S_{S L}\left(\mathbf{T}_{S L}\right)\right\}<\min \left\{T_{W L, k}: k \in \mathcal{S}_{W L}\left(\mathbf{T}_{W L}\right)\right\} \text {, with } \\
& S_{S L}\left(\mathbf{T}_{S L}\right)=\left\{k: T_{S L, k}<\text { TMPSL }_{k}(t M I N)\right\} \text { and } \\
& S_{W L}\left(\mathbf{T}_{W L}\right)=\left\{j: T_{W L, j}<T M P W L_{j}(t M I N)\right\} \\
& =1 \text { if } \operatorname{TMPSL}_{k}(t M I N) \leq T_{S L, k} \text { for } k=1,2, \ldots, n S L \text { and } \\
& \min \left\{{T M P S L_{k}^{-1}}^{-1}\left(T_{S L, k}\right), k=1,2, \ldots, n S L\right\}<\min \left\{T M P W L^{-1}\left(T_{W L, j}\right), j=1,2, \ldots, n W L\right\}, \\
& \text { with } T M P S L_{k}^{-1}\left(T_{S L, k}\right)=\infty \text { if } T_{S L, k}>T M P S L_{k}(t M A X) \text { and } \\
& T M P W L_{j}^{-1}\left(T_{W L, j}\right)=\infty \text { if } T_{W L, k}>T M P W L_{j}(t M A X) \\
& =0 \text { otherwise, }
\end{aligned}
$$

and the iterated integrals involve integration over $T_{S L, k}$ for $k=1,2, \ldots, n S L$ and over $T_{W L, j}$ for $j=1,2, \ldots, n W L$. 
The role of the indicator function $\delta\left(\mathbf{T}_{W L}, \mathbf{T}_{S L}\right)$ defined in Eqs. (3.23) - (3.25) is to "pick out" the failure temperature pairs $\left(\mathbf{T}_{W L}, \mathbf{T}_{S L}\right)$ in which at least one SL fails before any WL. In particular, (i) the assignment in Eq. (3.23) picks out the pairs in which at least one SL fails before the start of the analysis at time $t M I N$ and, for the special case with one or more WLs also failing before $t M I N$, in which the minimum of the SL failure temperatures is less than the minimum of the WL failure temperatures for the links failing before $t M I N$; (ii) the assignment in Eq. (3.24) picks out the pairs in which at least one SL fails between $t M I N$ and $t M A X$ before the failure of any of the WLs; and (iii) the assignment in Eq. (3.25) removes the probability associated with pairs in which none of the SLs fail before any of the WLs from incorporation into $p F$.

The integral defining $p F$ in Eq. (3.22) can be approximated by

$$
p F \cong \sum_{i=1}^{N} \delta\left(\mathbf{T}_{W L, i}, \mathbf{T}_{S L, i}\right) / N,
$$

where

$$
\left[\mathbf{T}_{W L, i}, \mathbf{T}_{S L, i}\right]=\left[T_{W L, 1, i}, T_{W L, 2, i}, \ldots, T_{W L, n W L, i}, T_{S L, 1, i}, T_{S L, 2, i}, \ldots, T_{S L, n S L, i}\right], i=1,2, \ldots, N
$$

is a random sample from the possible values of $\mathbf{T}_{W L}$ and $\mathbf{T}_{S L}$ generated in consistency with the distributions defined by the density functions $f W L_{j}\left(T_{W L}\right), j=1,2, \ldots, n W L$, and $f S L_{k}\left(T_{S L}\right), k=1,2, \ldots, n S L$.

As previously indicated in conjunction with Eqs. (2.58) and (2.59) for one WL and one SL, importance sampling can be used to accelerate the convergence of Monte Carlo approximations to $p F$ obtained from the integral in Eq. (3.17). In particular, the representation for $p F$ defined by this integral can be reformulated as

$$
\begin{aligned}
p F=\int_{-\infty}^{\infty} \cdots \int_{-\infty}^{\infty} \int_{-\infty}^{\infty} \cdots \int_{-\infty}^{\infty}\left[\frac{\delta\left(\mathbf{T}_{W L}, \mathbf{T}_{S L}\right) \prod_{j=1}^{n W L} f W L_{j}\left(T_{W L, j}\right) \prod_{k=1}^{n S L} f S L_{k}\left(T_{S L, k}\right)}{\prod_{j=1}^{n W L} f I W L_{j}\left(T_{W L, j}\right) \prod_{k=1}^{n S L} f I S L_{k}\left(T_{S L, k}\right)}\right] \\
\cdot \prod_{j=1}^{n W L} f I W L_{j}\left(T_{W L, j}\right) \prod_{k=1}^{n S L} f I S L_{k}\left(T_{S L, k}\right) \prod_{j=1}^{n W L} \mathrm{~d} T_{W L, j} \prod_{k=1}^{n S L} \mathrm{~d} T_{S L, k}
\end{aligned}
$$

where $f I W L_{j}\left(T_{W L, j}\right), j=1,2, \ldots, n W L$, and $f I S L\left(T_{S L, k}\right), k=1,2, \ldots, n S L$, are density functions that correspond to the distribution functions used for importance sampling on the WL and SL failure temperature distributions.

The resulting importance sampling approximation of $p F$ is given by

$$
p F \cong \sum_{i=1}^{N}\left[\frac{\delta\left(\mathbf{T}_{W L, i}, \mathbf{T}_{S L, i}\right) \prod_{j=1}^{n W L} f W L_{j}\left(T_{W L, j, i}\right) \prod_{k=1}^{n S L} f S L_{k}\left(T_{S L, k, i}\right)}{\prod_{j=1}^{n W L} f I W L\left(T_{W L, j, i}\right) \prod_{k=1}^{n S L} f I S L\left(T_{S L, k, i}\right)}\right] / N,
$$


where [ $\left.\mathbf{T}_{W L, i}, \mathbf{T}_{S L, i}\right], i=1,2, \ldots, N$, is a sample of the form indicated in Eq. (3.27) but now generated in consistency with the distributions defined by $f I W L_{j}\left(T_{W L, j}\right)$ and $f I S L_{k}\left(T_{S L, k}\right)$ rather than the distributions defined by $f W L\left(T_{W L, j}\right)$ and $f S L\left(T_{S L, k}\right)$.

\subsection{Numerical Evaluation: Example}

The following example involves two WLs and two SLs, with failure assumed to occur if either SL fails before either WL fails. For illustration, the WL and SL temperature curves are assumed to have the same general shapes as in Eqs. (2.66) and (2.67), with some modifications to the coefficients so that the individual links will have different temperature curves. In particular,

$$
\begin{aligned}
& \operatorname{TMPWL}_{j}(t)=c_{1}+\left[c_{2}+c_{3 j} e^{-c_{4 j} t} \sin \left(c_{5 j} t\right)\right] \tanh \left(c_{6 j} t\right) \\
& \operatorname{TMPSL}_{k}(t)=c_{1}+c_{2} \tanh \left[c_{62}\left(1+c_{7 k}\right) t\right],
\end{aligned}
$$

where $c_{1}$ and $c_{2}$ are defined the same as in conjunction with Eqs. (2.66) and (2.67) (i.e., $\left.c_{1}=10^{\circ} \mathrm{C}, c_{2}=900^{\circ} \mathrm{C}\right), c_{31}$ $=-900^{\circ} \mathrm{C}, c_{32}=-1100^{\circ} \mathrm{C}, c_{41}=0.25 \mathrm{~min}^{-1}, c_{42}=0.30 \mathrm{~min}^{-1}, c_{51}=0.12 \mathrm{~min}^{-1}, c_{52}=0.18 \mathrm{~min}^{-1}, c_{61}=0.02 \mathrm{~min}^{-1}$, $c_{62}=0.04 \mathrm{~min}^{-1}, c_{71}=0.5$ and $c_{72}=0.8$ (Fig. 3.1). Use of the term $c_{62}\left(1+c_{7 k}\right)$ in the definition of $T M P S L_{k}(t)$ in Eq. (3.26) results in the temperature behavior of the two SLs being related to the temperature behavior of WL2 but unrelated to the temperature behavior of WL1.

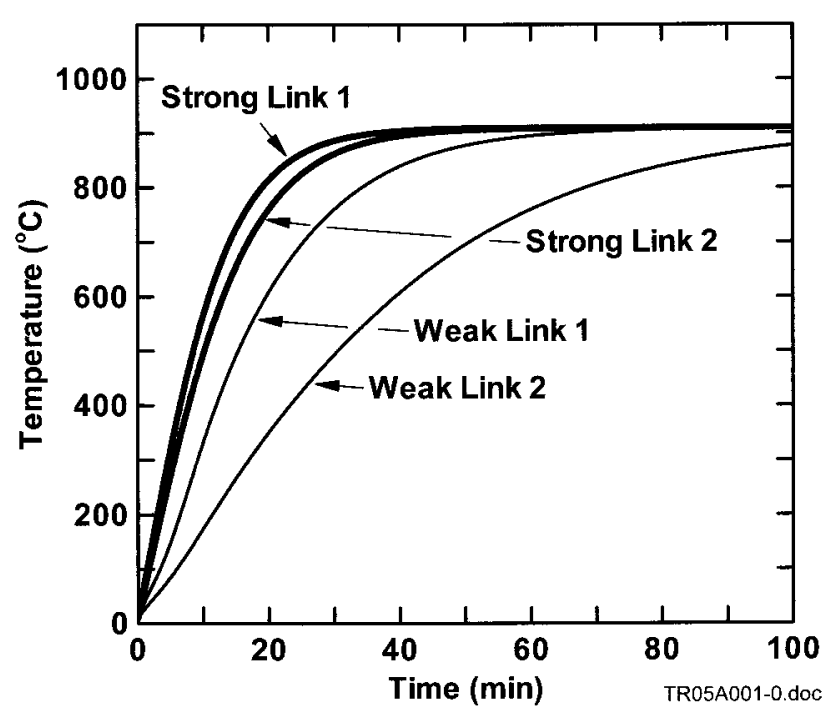

Fig. 3.1. Time-temperature curves defined in Eqs. (3.30) and (3.31) and used to illustrate calculation of failure probability $p F$ for two WLs and two SLs. 
Further, the density functions $f W L_{j}\left(T_{W L}\right)$ and $f S L_{j}\left(T_{S L}\right)$ for failure temperature are defined as in Eqs. (2.68) and (2.69), respectively. Specifically, $c_{8}=310^{\circ} \mathrm{C}$ and $c_{9}=8^{\circ} \mathrm{C}$ in the definition of $f W L_{1}\left(T_{W L}\right)$ and $f W L_{2}\left(T_{W L}\right)$, and $c_{10}=$ $560^{\circ} \mathrm{C}$ and $c_{11}=18^{\circ} \mathrm{C}$ in the definition of $f S L_{1}\left(T_{S L}\right)$ and $f S L_{2}\left(T_{S L}\right)$. However, although the distributions defined by $f W L_{1}\left(T_{W L}\right)$ and $f W L_{2}\left(T_{W L}\right)$ are the same, the failure temperatures for the two WLs are assumed to be independent; the same assumption is also made for the SL failure temperatures. The integral representations for $p F$ developed in this presentation are predicated on the assumption that the failure temperatures are independent. The assumption of dependence would result in more complex integral representations for $p F$. However, it would be relatively easy to modify the Monte Carlo calculations of $p F$ to incorporate correlations involving the failure temperatures.

The failure probability example introduced in this section is evaluated with the trapezoidal rule, Simpson's rule, simple random sampling and importance sampling (Table 3.1). The evaluations with the trapezoidal rule (see Eq. (2.49)) and Simpson's rule (see Eq. (2.50)) use the representations for $p F$ associated with Eqs. (3.14) and (3.20). The Monte Carlo evaluations based on simple random sampling and importance sampling use the representations for $p F$ in Eqs. (3.22) and (3.28), respectively. Further, the importance sampling evaluation uses uniform distributions with density functions $f I W L_{j}\left(T_{W L}\right)$ and $f I S L_{k}\left(T_{S L}\right)$ defined the same as $f I W L\left(T_{W L}\right)$ and $f I S L\left(T_{S L}\right)$ in Eqs. (2.68) and (2.69), respectively. The four approaches produce similar values for $p F$, although the two sampling based approaches require larger numbers of function evaluations. 
Table 3.1. Approximation of Failure Probability $p F$ for System Defined in Sect. 3.4 with Two WLs, Two SLs, Normal Distributions for WL and SL Failure Temperatures, and Failure of a SL Before Either WL Constituting System Failure (i.e., the Failure Configuration Described in Sect. 3.1) $)^{\mathrm{a}}$

\begin{tabular}{|c|c|c|c|c|c|c|}
\hline & $\mathrm{N}^{\mathrm{b}}$ & $\begin{array}{l}\text { Trapezoidal } \\
\text { Rule }^{\mathrm{c}}\end{array}$ & $\begin{array}{l}\text { Simpson's } \\
\text { Rule }^{\mathrm{d}}\end{array}$ & $\mathrm{N}^{\mathrm{e}}$ & $\begin{array}{l}\text { Random } \\
\text { Sampling }\end{array}$ & $\begin{array}{l}\text { Importance } \\
\text { Sampling }\end{array}$ \\
\hline & 3 & $3.623 \mathrm{E}-06$ & $4.831 \mathrm{E}-06$ & 1,000 & $1.830 \mathrm{E}-01$ & $2.629 \mathrm{E}-05$ \\
\hline & 5 & $1.812 \mathrm{E}-06$ & $1.208 \mathrm{E}-06$ & 10,000 & $1.690 \mathrm{E}-01$ & $2.250 \mathrm{E}-01$ \\
\hline & 9 & $4.086 \mathrm{E}-04$ & $5.442 \mathrm{E}-04$ & 100,000 & $1.668 \mathrm{E}-01$ & $1.604 \mathrm{E}-01$ \\
\hline & 17 & $6.489 \mathrm{E}-02$ & $8.639 \mathrm{E}-02$ & $1,000,000$ & $1.679 \mathrm{E}-01$ & $1.648 \mathrm{E}-01$ \\
\hline & 33 & $1.781 \mathrm{E}-01$ & $2.159 \mathrm{E}-01$ & $10,000,000$ & $1.679 \mathrm{E}-01$ & $1.760 \mathrm{E}-01$ \\
\hline & 65 & $1.679 \mathrm{E}-01$ & $1.645 \mathrm{E}-01$ & $100,000,000$ & $1.679 \mathrm{E}-01$ & $1.666 \mathrm{E}-01$ \\
\hline & 129 & $1.678 \mathrm{E}-01$ & $1.678 \mathrm{E}-01$ & & & \\
\hline & 257 & $1.678 \mathrm{E}-01$ & $1.678 \mathrm{E}-01$ & & & \\
\hline & 513 & --- & $1.678 \mathrm{E}-01$ & & & \\
\hline \multicolumn{7}{|c|}{ a Calculations performed with CPLOAS program (App. III). } \\
\hline \multicolumn{7}{|c|}{$\begin{array}{l}\text { Number of evaluations of } G\left(T_{S L}\right) \text { (see Eq. (3.14)) with trapezoidal rule and Simpson's rule, which corresponds to the interval }[T M N S L \text {, } \\
T M X S L] \text { being divided into } N-1 \text { subintervals. }\end{array}$} \\
\hline $\mathrm{c}$ & \multicolumn{6}{|c|}{ Approximation to $p F$ obtained with trapezoidal rule (see Eq. (2.49)). } \\
\hline e & \multicolumn{6}{|c|}{ Approximation to $p F$ obtained with Simpson's rule (see Eq. (2.50)). } \\
\hline e & \multicolumn{6}{|c|}{ Number of evaluation of $\delta\left(\mathbf{T}_{W L}, \mathbf{T}_{S L}\right)=\delta\left(T_{W L, 1}, T_{W L, 2}, T_{S L, 1}, T_{S L, 2}\right)$ (see Eqs. (3.23) - (3.25)) for random sampling and importance sampling. } \\
\hline f & \multicolumn{6}{|c|}{ Approximation to $p F$ obtained with random sampling (see Eq. (3.26)). } \\
\hline g & \multicolumn{6}{|c|}{$\begin{array}{l}\text { Approximation to } p F \text { obtained with importance sampling with uniform distributions for } T_{W L, 1}, T_{W L, 2}, T_{S L, 1} \text { and } T_{S L, 2} \text { (see Eqs. (3.28) and } \\
\text { (3.29), with } f I W L_{j}\left(T_{W L_{j}}\right) \text { and } f I S L_{k}\left(T_{S L, k}\right) \text { defined as indicated in Eqs. (2.70) and (2.71), respectively). }\end{array}$} \\
\hline
\end{tabular}


This page intentionally left blank. 


\section{Multiple WLs and SLs with Failure of All SLs Before Any WL Constituting System Failure}

The analysis of PLOAS for a system involving multiple WLs and multiple SLs with failure of all SLs before failure of any WL constituting system failure is now presented. The following topics are considered: formal mathematical representation of PLOAS (Sect. 4.1), numerical evaluation of defining integral for PLOAS with quadrature methods (Sect. 4.2), numerical evaluation of defining integral for PLOAS with Monte Carlo methods (Sect. 4.3), example of the numerical evaluation of PLOAS (Sect. 4.4), and comparison with a representation for PLOAS developed by M.P. Bohn for one WL and two SLs (Sect. 4.5).

\subsection{Formal Representation}

The system is assumed to involve $n W L$ WLs and $n S L$ SLs as in Sect. 3. However, the system is now assumed to fail only if all SLs fail before any WL fails. The functions $T M P W L_{j}, T M P S L_{k}, f W L_{j}$ and $f S L_{k}$ are the same as in Eqs. $(3.1)-(3.4)$.

The underlying sample space $S$ is the same as defined in Eq. (3.5). However, the set $\mathcal{E}$ used in the definition of $p F$ is different. In particular,

$$
p F=\operatorname{prob}(\mathcal{E})
$$

where $\mathcal{E}$ is now the subset of $\mathcal{S}$ defined by

$$
\begin{aligned}
& \mathcal{E}=\left\{\mathbf{t}: \mathbf{t}=\left[t F S L_{1}, t F S L_{2}, \ldots, t F S L_{n S L}, t F W L_{1}, t F W L_{2}, \ldots, t F W L_{n W L}\right] \in \mathcal{S} \text { and } t F S L_{k}<t F W L_{j}\right. \\
& \quad \text { for } k=1,2, \ldots, n S L, j=1,2, \ldots, n W L\} .
\end{aligned}
$$

Specifically, $\mathcal{E}$ contains the elements of $S$ for which all SLs fail before any WL fails.

The determination of $p F$ is based on the following decomposition of $\mathcal{E}$ :

$$
\varepsilon=\bigcup_{i=1}^{n T M}\left[\bigcup_{k=1}^{n S L}\left(\varepsilon_{i k} \cup \tilde{\varepsilon}_{i k}\right)\right],
$$

where

$$
\begin{aligned}
\mathcal{E}_{i k}= & \left\{\mathbf{t}: \mathbf{t} \in \mathcal{E}, t_{i-1} \leq t F S L_{k} \leq t_{i}, t F S L_{l}<t_{i-1} \text { for } l \neq k, t_{i}<t F W L_{j} \text { for } j=1,2, \ldots, n W L\right\}, \\
\tilde{\varepsilon}_{i k}= & \left\{\mathbf{t}: \mathbf{t} \in \mathcal{E}, t_{i-1} \leq t F S L_{k} \leq t_{i}, t F S L_{l} \leq t F S L_{k} \text { for } l \neq k, t F S L_{k} \leq t F W L_{j} \text { for } j=1,2, \ldots, n W L\right. \text { and either (i) there } \\
& \text { exists at least one value of } l \text { such that } t_{i-1} \leq t F S L_{l} \text { or (ii) there exists at least one value of } j \text { such that } \\
& \left.t F W L_{j} \leq t_{i}\right\},
\end{aligned}
$$


and $t M I N=t_{0}<t_{1}<\ldots<t_{n T M}=t M A X$ is a partition of $[t M I N, t M A X]$ as indicated in Fig. 2.1. The set $\tilde{\varepsilon}_{i k}$ contains elements of $\mathcal{E}$ that have a SL failure time $t F S L_{l}, l \neq k$, satisfying $t_{i-1} \leq t F S L_{l} \leq t F S L_{k}$ or a WL failure time $t F W L_{j}, j=$ $1,2, \ldots, n W L$, satisfying $t F S L_{k}<t F W L_{j} \leq t_{i}$. The equality

$$
\operatorname{prob}(\mathcal{E})=\sum_{i=1}^{n T M} \sum_{k=1}^{n S L} \operatorname{prob}\left(\varepsilon_{i k}\right)+\sum_{i=1}^{n T M} \sum_{k=1}^{n S L} \operatorname{prob}\left(\tilde{\varepsilon}_{i k}\right)
$$

holds because the sets involved are disjoint (see parenthetical remark following Eq. (3.9)).

The equality

$$
\lim _{n T M \rightarrow \infty} \sum_{i=1}^{n T M} \sum_{k=1}^{n S L} \operatorname{prob}\left(\tilde{\varepsilon}_{i k}\right)=0
$$

holds because the indicated probabilities involve products whose individual terms are proportional to $\Delta t_{i}$ (see Eq. (2.9) for an analogous situation). Further, similarly to Eq. (3.11),

$$
\begin{aligned}
\operatorname{prob}\left(\varepsilon_{i k}\right)= & I\left[\operatorname{TMPSL}_{k}\left(t_{i-1}\right), \operatorname{TMPSL}_{k}\left(t_{i}\right), f S L_{k}\right] \\
& \cdot \prod_{\substack{l=1 \\
l \neq k}}^{n S L} I\left[-\infty, \operatorname{TMPSL}_{l}\left(t_{i-1}\right), f S L_{l}\right] \prod_{j=1}^{n W L} I\left[T M P W L_{j}\left(t_{i}\right), \infty, f W L_{j}\right] \\
\cong & \left\{\left[\operatorname{TMPSL}_{k}\left(t_{i}\right)-\operatorname{TMPSL}_{k}\left(t_{i-1}\right)\right] f S L_{k}\left[T M P S L_{k}\left(t_{i}\right)\right]\right\} \\
& \cdot \prod_{\substack{l=1 \\
l \neq k}}^{n S L} I\left[-\infty, \operatorname{TMPSL}_{l}\left(t_{i-1}\right), f S L_{l}\right] \prod_{j=1}^{n W L} I\left[T M P W L_{j}\left(t_{i}\right), \infty, f W L_{j}\right]
\end{aligned}
$$

where (i) $I[a, b, f]$ is defined in conjunction with Eqs. (2.9) and (2.10), (ii) the first term in the preceding products corresponds to the probability that SL $k$ will fail in the time interval $\left[t_{i-1}, t_{i}\right]$, (iii) the second term corresponds to the probability that all SLs except SL $k$ will fail before $t_{i-1}$, and (iv) the third term corresponds to the probability that all WLs will fail after $t_{i}$.

As a result, 


$$
\begin{aligned}
\operatorname{prob}(\mathcal{E})= & \lim _{n T M \rightarrow \infty} \sum_{i=1}^{n T M} \sum_{k=1}^{n S L} \operatorname{prob}\left(\mathcal{E}_{i k}\right)+\lim _{n T M \rightarrow \infty} \sum_{i=1}^{n T M} \sum_{k=1}^{n S L} \operatorname{prob}\left(\tilde{\varepsilon}_{i k}\right) \\
= & \lim _{n T M \rightarrow \infty} \sum_{i=1}^{n T M} \sum_{k=1}^{n S L} \operatorname{prob}\left(\mathcal{E}_{i k}\right)+0 \\
= & \lim _{n T M \rightarrow \infty} \sum_{i=1}^{n T M} \sum_{k=1}^{n S L}\left\{\left[T M P S L_{k}(t)-\operatorname{TMPSL}_{k}\left(t_{i-1}\right)\right] f S L_{k}\left[T M P S L_{k}\left(t_{i}\right)\right]\right\} \\
& \quad \prod_{\substack{l=1 \\
l \neq k}}^{n S L} I\left[-\infty, \operatorname{TMPSL}_{l}\left(t_{i-1}\right), f S L_{l}\right] \prod_{j=1}^{n W L} I\left[T M P W L_{j}\left(t_{i}\right), \infty, f W L_{j}\right],
\end{aligned}
$$

where the first, second and third equalities follow from Eqs. (4.4), (4.5) and (4.6), respectively. Evaluation of the final limit in Eq. (4.7) leads to the representation of $\operatorname{prob}(\mathcal{E})$, and hence $p F$, by

$$
\begin{aligned}
p F=\sum_{k=1}^{n S L}\left(\int_{t M I N}^{t M A X}\left\{f S L_{k}\left[T M P S L_{k}(t)\right]\right\}\left\{\prod_{\substack{l=1 \\
l \neq k}}^{n S L} I\left[-\infty, T M P S L_{l}(t), f S L_{l}\right]\right\}\right. \\
\left.\cdot\left\{\prod_{j=1}^{n W L} I\left[T M P W L_{j}(t), \infty, f W L_{j}\right]\right\} \mathrm{d} T M P S L_{k}(t)\right) \\
=\int_{t M I N}^{t M A X}\left(\sum_{k=1}^{n S L}\left\{f S L_{k}\left[T M P S L_{k}(t)\right]\right\}\left\{\begin{array}{l}
n S L \\
\left.\left.\prod_{l=1} I\left[-\infty, T M P S L_{l}(t), f S L_{l}\right]\right\}\left\{\mathrm{d} T M P S L_{k}(t) / \mathrm{d} t\right\}\right) \\
l \neq k
\end{array}\right\}\right. \\
\cdot\left(\prod_{j=1}^{n W L} I\left[T M P W L_{j}(t), \infty, f W L_{j}\right]\right) \mathrm{d} t,
\end{aligned}
$$

where the first integral is a Riemann-Stieltjes integral and the second integral is the corresponding Riemann integral.

The Riemann integral defining $p F$ in Eq. (4.8) is with respect to time. As discussed in conjunction with Eqs. (2.16) and (2.17), the definition of $p F$ can be converted to an integral with respect to temperature by a suitable change of variable. This change produces

$$
\begin{aligned}
p F= & \sum_{k=1}^{n S L} \int_{T_{M N S L_{k}}}^{T M X S L_{k}}\left(\left\{f S L_{k}\left(T_{S L}\right)\right\}\left\{\prod_{\substack{l=1 \\
l \neq k}}^{n S L} I\left[-\infty, T M P S L_{l}\left[T M P S L_{k}^{-1}\left(T_{S L}\right)\right], f S L_{l}\right]\right\}\right. \\
& \left.\cdot\left\{\prod_{j=1}^{n W L} I\left[T M P W L_{j}\left[T^{\prime} M P S L_{k}^{-1}\left(T_{S L}\right)\right], \infty, f W L_{j}\right]\right\}\right) \mathrm{d} T_{S L} \\
= & \int_{T M N S L}^{T M X S L} G\left(T_{S L}\right) \mathrm{d} T_{S L},
\end{aligned}
$$

where notation is the same as in Eq. (3.14) except that now 


$$
\begin{aligned}
G_{k}\left(T_{S L}\right)= & f S L_{k}\left(T_{S L}\right)\left\{\prod_{\substack{l=1 \\
l \neq k}}^{n S L} I\left[-\infty, T M P S L_{l}\left[T M P S L_{k}^{-1}\left(T_{S L}\right)\right], f S L_{l}\right]\right\} \\
& \cdot\left\{\prod_{j=1}^{n W L} I\left[T M P W L_{j}\left[T M P S L_{k}^{-1}\left(T_{S L}\right)\right], \infty, f W L_{j}\right]\right\} \text { for } T M N S L_{k} \leq T_{S L} \leq T M X S L_{k} \\
= & 0 \quad \text { otherwise }
\end{aligned}
$$

in the definition of $G\left(T_{S L}\right)$. The simpler representation

$$
\begin{aligned}
p F=\int_{T M N S L}^{T M X S L}\left(\sum_{k=1}^{n S L}\left\{f S L_{k}\left(T_{S L}\right)\right\}\left\{\prod_{\substack{l=1 \\
l \neq k}}^{n S L} I\left[-\infty, T M P S L_{l}\left[T M P S L_{k}^{-1}\left(T_{S L}\right)\right], f S L_{l}\right]\right\}\right. \\
\left.\cdot\left\{\prod_{j=1}^{n W L} I\left[T M P W L_{j}\left[\operatorname{TMPSL}_{k}^{-1}\left(T_{S L}\right)\right], \infty, f W L_{j}\right]\right\}\right) \mathrm{d} T_{S L}
\end{aligned}
$$

results for the first equality in Eq. (4.9) when $T M N S L_{k}=T M N S L$ and $T M X S L_{k}=T M X S L$ for $k=1,2, \ldots, n S L$.

\subsection{Numerical Evaluation: Quadrature}

The same numerical procedures indicated in Sect. 3.2 (i.e., the quadrature formulas in Eqs. (2.49) and (2.50)) can be used to determine the failure probability $p F$ for the WL/SL configuration described in the preceding section (Sect. 4.1). The only difference is a change in the definition of the function $G\left(T_{S L}\right)$ resulting from a changed definition for $G_{k}\left(T_{S L}\right)$ (see Eqs. (3.19), (3.20), (4.10)).

Evaluation of $G\left(T_{S L}\right)$ in conjunction with Eq. (4.19) involves integrals of both the forms $I[c, \infty, f]$ and $I[-\infty, c$, $f$, where $f$ is a probability density function (see definition of $G_{k}\left(T_{S L}\right)$ in Eq. (4.10)). The evaluation of $\left.I c, \infty, f\right]$ is discussed in Eqs. (2.36) - (2.48) for uniform, loguniform, normal and lognormal distributions (i.e., for the density functions $f_{u}, f_{l u}, f_{n}$ and $f_{l n}$ in the notation used in conjunction with Eqs. (2.36) - (2.48)). Similar evaluations are also possible for $I[-\infty, c, f]$. Specifically,

$$
I\left[-\infty, c, f_{u}\right]= \begin{cases}0 & \text { if } c \leq a \\ (c-a) /(b-a) & \text { if } a \leq c \leq b \\ 1 & \text { if } c \geq b\end{cases}
$$

for $-\infty<c<\infty$ and the uniform density function $f_{u}$ defined in Eq. (2.30); 


$$
I\left[-\infty, c, f_{l u}\right]= \begin{cases}0 & \text { if } c \leq a \\ \ln (c / a) / \ln (b / a) & \text { if } a \leq c \leq b \\ 1 & \text { if } c \geq b\end{cases}
$$

for $-\infty<c<\infty$ and the loguniform density function $f_{l u}$ defined in Eq. (2.31);

$$
I\left[-\infty, c, f_{n}\right]=\int_{-\infty}^{c}(1 / \sigma \sqrt{2 \pi}) \exp \left[-(T-\mu)^{2} / 2 \sigma^{2}\right] \mathrm{d} T
$$

for $-\infty<c<\infty$ and the normal density function $f_{n}$ defined in Eq. (2.32), and

$$
I\left[-\infty, c, f_{l n}\right]=\left\{\begin{array}{lr}
0 & \text { if } c \leq 0 \\
\int_{0}^{c}(1 / T \sqrt{2 \pi}) \exp \left[-(\ln T-\mu)^{2} / 2 \sigma^{2}\right] \mathrm{d} T & \\
=\int_{0}^{\ln c}(1 / \sigma \sqrt{2 \pi}) \exp \left[-(y-\mu)^{2} / 2 \sigma^{2}\right] \mathrm{d} y & \text { if } c>0
\end{array}\right.
$$

for $-\infty<c<\infty$ and the lognormal density function $f_{l n}$ defined in Eq. (2.33) with $f_{l n}(T)=0$ assumed for $T \leq 0$.

Similarly to $I\left[c, \infty, f_{n}\right]$ and $\left.I c, \infty, f_{l n}\right]$ as discussed in conjunction with Eqs. (2.40) - (2.48), closed form representations for $I\left[-\infty, c, f_{n}\right]$ and $I\left[-\infty, c, f_{l n}\right]$ do not exist. However, $I\left[-\infty, c, f_{n}\right]$ and $I\left[-\infty, c, f_{l n}\right]$ can be represented by

$$
I\left[-\infty, c, f_{n}\right]=P[(c-\mu) / \sigma]
$$

and

$$
I\left[-\infty, c, f_{l n}\right]= \begin{cases}0 & \text { if } c \leq 0 \\ P[(\ln c-\mu) / \sigma] & \text { if } c>0,\end{cases}
$$

where $P(x)$ denotes the Gaussian probability integral defined in Eq. (2.42). In turn, $P(x)$ can be approximated by the relation in Eq. (2.43).

An alternative approximation can be obtained from the representations

$$
I\left[-\infty, c, f_{n}\right]=1-I\left[c, \infty, f_{n}\right]=1-(1 / 2) \operatorname{erfc}[(c-\mu) / \sigma \sqrt{2}]
$$

and

$$
I\left[-\infty, c, f_{l n}\right]=1-I\left[c, \infty, f_{l n}\right]=1-1 / 2 \operatorname{erfc}[(\ln c-\mu) / \sigma \sqrt{2}], c>0
$$

that result from Eqs. (2.44) and (2.45). Then, approximations to $I\left[-\infty, c, f_{n}\right]$ and $I\left[-\infty, c, f_{l n}\right]$ follow from the representations for $\operatorname{erfc}(x)$ in Eqs. (2.47) and (2.48). 


\subsection{Numerical Evaluation: Monte Carlo}

The same Monte Carlo procedures indicated in Sect. 3.3 can be used to determine the failure probability $p F$ for the WL/SL configuration described in Sect. 4.1. The only difference is in the definition of the indicator function $\delta\left(\mathbf{T}_{W L}, \mathbf{T}_{S L}\right)$ in Eq. (3.22). For the WL/SL configuration described in Sect. 4.1, $\delta\left(\mathbf{T}_{W L}, \mathbf{T}_{S L}\right)$ is defined by

$$
\begin{aligned}
& \delta\left(\mathbf{T}_{W L}, \mathbf{T}_{S L}\right)=\delta\left(T_{W L, 1}, T_{W L, 2}, \ldots, T_{W L, n W L}, T_{S L, 1}, T_{S L, 2}, \ldots, T_{S L, n S L}\right) \\
& =1 \text { if } T_{S L, k}<T M P S L_{k}(t M I N) \text { for } k=1,2, \ldots, n S L \text { and } \max \left\{T_{S L, k}: k=1,2, \ldots, n S L\right\}< \\
& \min \left\{T_{W L, j}: 1 \leq j \leq n W L \text { and } T_{W L, j}<T M P W L_{j}(t M I N)\right\} \\
& =1 \text { if } S_{S L}\left(\mathbf{T}_{S L}\right) \neq \phi, S_{W L}\left(\mathbf{T}_{W L}\right)=\phi \text { and } \max \left\{\operatorname{TMPSL}_{k}^{-1}\left(T_{S L, k}\right): k \in S_{S L}\left(\mathbf{T}_{S L}\right)\right\}< \\
& \min \left\{T M P W L^{-1}\left(T_{W L, j}\right): j=1,2, \ldots, n W L\right\} \text {, where } \\
& S_{S L}\left(\mathbf{T}_{S L}\right)=\left\{k: T_{M P S L_{k}}(t M I N) \leq T_{S L, k}\right\}, \quad S_{W L}\left(\mathbf{T}_{W L}\right)=\left\{j: T_{W L, j}<T M P W L_{j}(t M I N)\right\}, \\
& \operatorname{TMPSL}_{k}^{-1}\left(T_{S L, k}\right)=\infty \text { if } T_{S L, k}>T M P S L_{k}(t M A X), \text { and } \\
& T M P W L_{j}^{-1}\left(T_{W L, j}\right)=\infty \text { if } T_{W L, j}>T M P W L_{j}(t M A X) \\
& =0 \text { otherwise. }
\end{aligned}
$$

With respect to the role of the indicator function $\delta\left(\mathbf{T}_{W L}, \mathbf{T}_{S L}\right)$, (i) the assignment in Eq. (4.20) picks out the pairs $\left(\mathbf{T}_{W L}, \mathbf{T}_{S L}\right)$ in which all SLs fail before the start of the analysis at time $t M I N$ and any WL failing before the start of the analysis has a higher failure temperature than any SL, (ii) the assignment in Eq. (4.21) picks out the pairs in which all SLs fail before the end of the analysis at $t M A X$ and also before the failure of any WL, and (iii) the assignment in Eq. (4.22) removes the probability associated with pairs in which one or more WLs fail before all of the SLs fail from incorporation into $p F$.

With the definition of $\delta\left(\mathbf{T}_{W L}, \mathbf{T}_{S L}\right)$ in Eqs. (4.20) - (4.22), the Monte Carlo approximation to $p F$ for the WL/SL configuration in Sect. 4.1 has the form in Eq. (3.26) when simple random sampling is used and the form in Eq. (3.29) when importance sampling is used.

\subsection{Numerical Example: Example}

The example introduced in Sect. 3.4 is also used to illustrate the WL/SL configuration described in Sect. 4.1 where all SLs are required to fail before any WL to produce a failure of the WL/SL system (Table 4.1). The only difference in the implementation of the numerical results is the use of the definition of $G_{k}\left(T_{S L}\right)$ in Eq. (4.10) instead of the definition in Eq. (3.20) in the generation of quadrature results (see Eqs. (2.49) - (2.50)) and the use of the definition $\delta\left(\mathbf{T}_{W L}, \mathbf{T}_{S L}\right)$ in Eqs. (4.20) - (4.22) instead of the definition in Eqs. (3.23) - (3.25) in the generation of 
Monte Carlo results with simple random sampling (see Eq. (3.26) and importance sampling (see Eq. (3.29)). The four approaches produce similar values for $p F$, with the two quadrature approaches requiring far fewer function evaluations than the two sampling approaches. Further, the requirement that both SLs fail before either WL fails results in a much smaller value for $p F$ (i.e., $p F \cong 1.6 \times 10^{-6}$ in Table 4.1) than is the case in the preceding section (Sect. 3) where only one SL is required to fail prior to the failure of either WL (i.e., $p F \cong 1.7 \times 10^{-1}$ in Table 3.1).

\subsection{Comparison with One WL, Two SL Representation Developed by Bohn}

For one WL and two SLs and with the notation used in his presentation, Bohn's representation for the probability $p F$ that both SLs fail before the WL for the system described in Sect. 4.1 is

$$
p F=\int_{t M I N}^{t M A X} C D F_{S L 1}(t) C D F_{S L 2}(t) P D F_{W L 1}(t) \mathrm{d} t
$$

Table 4.1. Approximation of Failure Probability pF for System Defined in Sect. 3.4 with Two WLs, Two SLs, Normal Distributions for WL and SL Failure Temperatures, and Failure of Both SLs before Either WL Constituting System Failure (i.e., the Failure Configuration Described in Sect. 4.1) $)^{a}$

\begin{tabular}{|c|c|c|c|c|c|c|}
\hline & $\mathrm{N}^{\mathrm{b}}$ & $\begin{array}{l}\text { Trapezoidal } \\
\text { Rule }^{\mathrm{c}}\end{array}$ & $\begin{array}{l}\text { Simpson's } \\
\text { Rule }^{\mathrm{d}}\end{array}$ & $\mathrm{N}^{\mathrm{e}}$ & $\begin{array}{l}\text { Random } \\
\text { Sampling }\end{array}$ & $\begin{array}{l}\text { Importance } \\
\text { Sampling } \mathrm{g}\end{array}$ \\
\hline & 3 & $8.273 \mathrm{E}-08$ & $1.103 \mathrm{E}-07$ & 1,000 & $0.000 \mathrm{E}+00$ & $2.185 \mathrm{E}-13$ \\
\hline & 5 & $4.136 \mathrm{E}-08$ & $2.758 \mathrm{E}-08$ & 10,000 & $0.000 \mathrm{E}+00$ & $5.521 \mathrm{E}-07$ \\
\hline & 9 & $2.076 \mathrm{E}-07$ & $2.630 \mathrm{E}-07$ & 100,000 & $0.000 \mathrm{E}+00$ & $2.226 \mathrm{E}-06$ \\
\hline & 17 & $1.058 \mathrm{E}-07$ & 7.183E-08 & $1,000,000$ & $2.000 \mathrm{E}-06$ & $1.968 \mathrm{E}-06$ \\
\hline & 33 & $2.052 \mathrm{E}-06$ & $2.700 \mathrm{E}-06$ & $10,000,000$ & $1.500 \mathrm{E}-06$ & $1.573 \mathrm{E}-06$ \\
\hline & 65 & $1.567 \mathrm{E}-06$ & $1.405 \mathrm{E}-06$ & $100,000,000$ & $1.670 \mathrm{E}-01$ & $1.594 \mathrm{E}-06$ \\
\hline & 129 & $1.557 \mathrm{E}-06$ & $1.553 \mathrm{E}-06$ & & & \\
\hline & 257 & $1.557 \mathrm{E}-06$ & $1.557 \mathrm{E}-06$ & & & \\
\hline & 513 & - & $1.557 \mathrm{E}-06$ & & & \\
\hline & 1025 & - & $1.557 \mathrm{E}-06$ & & & \\
\hline & 2049 & - & $1.557 \mathrm{E}-06$ & & & \\
\hline a & \multicolumn{6}{|c|}{ Calculations performed with CPLOAS program (App. III) } \\
\hline b & \multicolumn{6}{|c|}{$\begin{array}{l}\text { Number of evaluations of } G\left(T_{S L}\right) \text { (see Eq. (4.9)) with trapezoidal rule and Simpson's rule, which corresponds to the interval [TMNSL, } \\
T M X S L] \text { being divided into } N-1 \text { subintervals. }\end{array}$} \\
\hline $\mathrm{c}$ & \multicolumn{6}{|c|}{ Approximation to $p F$ obtained with trapezoidal rule (see Eq. (2.49)). } \\
\hline $\mathrm{d}$ & \multicolumn{6}{|c|}{ Approximation to $p F$ obtained with Simpson's rule (see Eq. (2.50)). } \\
\hline e & \multicolumn{6}{|c|}{ Number of evaluation of $\delta\left(\mathbf{T}_{W L}, \mathbf{T}_{S L}\right)=\delta\left(T_{W L, 1}, T_{W L, 2}, T_{S L, 1}, T_{S L, 2}\right)$ (see Eqs. (4.20) - (4.22)) for random sampling and importance sampling. } \\
\hline f & \multicolumn{6}{|c|}{ Approximation to $p F$ obtained with random sampling (see Eq. (3.26)). } \\
\hline g & \multicolumn{6}{|c|}{$\begin{array}{l}\text { Approximation to } p F \text { obtained with importance sampling with uniform distributions for } T_{W L, 1}, T_{W L, 2}, T_{S L, 1} \text { and } T_{S L, 2} \text {, (see Eqs. (3.28) and } \\
\text { (3.29), with } f I W L_{j}\left(T_{W L, j}\right) \text { and } f I S L_{k}\left(T_{S L, k}\right) \text { defined as indicated in Eqs. (2.70) and (2.71), respectively). }\end{array}$} \\
\hline
\end{tabular}


where (i) $C D F_{S L 1}(t)$ and $C D F_{S L 2}(t)$ are the probabilities that SLs 1 and 2, respectively, fail before time $t$, (ii) $P D F_{W L 1}(t)$ is defined the same as in Eq. (2.72), and (iii) $[t M I N, t M A X]$ is the time interval over which the calculation is carried out. This is the representation given in Eq. (4) of Bohn's report with the time interval $[0, \infty]$ replaced by $[t M I N, t M A X]$ and $t_{0}$ replaced by $t$ for notational convenience.

In the notation used in this presentation, the representation for $p F$ in Eq. (4.23) becomes

$$
\begin{gathered}
p F=\int_{t M I N}^{t M A X}\left(\prod_{k=1}^{2}\left[\int_{t M I N}^{t}\left\{f S L_{k}\left[T M P S L_{k}(\tau)\right]\right\}\left\{\mathrm{d} T M P S L_{k}(\tau) / \mathrm{d} \tau\right\} \mathrm{d} \tau\right]\right) \\
\cdot(\{f W L[T M P W L(t)]\}\{\mathrm{d} T M P W L(t) / \mathrm{d} t\}) \mathrm{d} t,
\end{gathered}
$$

where (i) $C D F_{S L 1}(t)$ and $C D F_{S L 2}(t)$ are defined analogously to $C D F_{S L 1}(t)$ in Eq. (2.73) with subscripts of $k=1,2$ used on $f S L_{k}$ and $T M P S L_{k}$ to distinguish between the two SLs and (ii) $P D F_{W L 1}(t)$ is defined the same as $P D F_{W L 1}(t)$ in Eq. (2.73) with no subscripting needed on $f W L$ and TMPWL as only one WL is involved. The corresponding representations for $p F$ given in Eqs. (4.8) and (4.9) are

$$
\begin{aligned}
p F= & \int_{t M I N}^{t M A X}\left(\left\{f S L_{1}\left[T M P S L_{1}(t)\right]\right\}\left\{\int_{-\infty}^{T M P S L_{2}(t)} f S L_{2}\left(T_{S L 2}\right) \mathrm{d} T_{S L 2}\right\}\left\{\mathrm{d} T M P S L_{1}(t) / \mathrm{d} t\right\}\right. \\
& \left.+\left\{f S L_{2}\left[T M P S L_{2}(t)\right]\right\}\left\{\int_{-\infty}^{T M P S L_{1}(t)} f S L_{1}\left(T_{S L 1}\right) \mathrm{d} T_{S L 1}\right\}\left\{\mathrm{d} T M P S L_{2}(t) / \mathrm{d} t\right\}\right)\left(\int_{T M P W L(t)}^{\infty} f W L\left(T_{W L}\right) \mathrm{d} T_{W L}\right) \mathrm{d} t \\
= & \int_{T M N S L_{1}}^{T M X S L_{1}} f S L_{1}\left(T_{S L 1}\right)\left\{\int_{-\infty}^{F S L_{21}\left(T_{S L 1}\right)} f S L_{2}\left(T_{S L 2}\right) \mathrm{d} T_{S L 2}\right\}\left\{\int_{F W L_{1}\left(T_{S L 1}\right)}^{\infty} f W L\left(T_{W L}\right) \mathrm{d} T_{W L}\right\} \mathrm{d} T_{S L 1} \\
& +\int_{T M N S L_{2}}^{T M X S L_{2}} f S L_{2}\left(T_{S L 2}\right)\left\{\int_{-\infty}^{F S L_{12}\left(T_{S L 2}\right)} f S L_{1}\left(T_{S L 1}\right) \mathrm{d} T_{S L 1}\right\}\left\{\int_{F W L_{2}\left(T_{S L 2}\right)}^{\infty} f W L\left(T_{W L}\right) \mathrm{d} T_{W L}\right\} \mathrm{d} T_{S L 2},
\end{aligned}
$$

where

$$
F S L_{k l}\left(T_{S L}\right)=\operatorname{TMPSL}_{k}\left[\operatorname{TMPSL}_{l}^{-1}\left(T_{S L}\right)\right]
$$

expresses the temperature of SL $k$ as a function of the temperature of SL $l$ and

$$
F W L_{k}\left(T_{S L}\right)=T M P W L\left[T M P S L_{k}^{-1}\left(T_{S L}\right)\right]
$$

expresses the temperature of the WL as a function of the temperature of SL $k$.

The equivalence of the representation for $p F$ in Eq. (4.24) and the representation for $p F$ in Eqs. (4.25) and (4.26) will be established by using suitable changes of variable (see Eq. (2.16)) to reformulate the representation for 
$p F$ in Eq. (4.24) into a form that can be shown to have the same value as the representation in Eq. (4.26). Specifically, Eq. (4.24) can be rewritten as

$$
\begin{aligned}
p F & =\int_{t M I N}^{t M A X}\left(\prod_{k=1}^{2}\left[\int_{T M N S L_{k}}^{T M P S L_{k}(t)} f S L_{k}\left(T_{S L k}\right) \mathrm{d} T_{S L k}\right]\right)(\{f W L[T M P W L(t)]\}\{\mathrm{d} T M P W L(t) / \mathrm{d} t\}) \mathrm{d} t \\
& =\int_{T M N W L}^{T M X W L}\left(\prod_{k=1}^{2}\left[\int_{T M N S L_{k}}^{F W L_{k}^{-1}\left(T_{W L}\right)} f S L_{k}\left(T_{S L k}\right) \mathrm{d} T_{S L k}\right]\right) f W L\left(T_{W L}\right) \mathrm{d} T_{W L} \\
& =\int_{T M N W L}^{T M X W L} \int_{T M N S L_{1}}^{F W L_{1}^{-1}\left(T_{W L}\right)} \int_{T M N S L_{2}}^{F}\left(T_{W L}\right) f S L_{2}\left(T_{S L 2}\right) f S L_{1}\left(T_{S L 1}\right) f W L\left(T_{W L}\right) \mathrm{d} T_{S L 2} \mathrm{~d} T_{S L 1} \mathrm{~d} T_{W L} \\
& =\int_{S} f\left(T_{W L}, T_{S L 1}, T_{S L 2}\right) \mathrm{d} V
\end{aligned}
$$

where

$$
\begin{aligned}
& T M N W L=T M P W L(t M I N), T M X W L=T M P W L(t M A X) \\
& f\left(T_{W L}, T_{S L 1}, T_{S L 2}\right)=f S L_{2}\left(T_{S L 2}\right) f S L_{1}\left(T_{S L 1}\right) f W L\left(T_{W L}\right) \\
& S=\left\{\left(T_{W L}, T_{S L 1}, T_{S L 2}\right): T M N W L \leq T_{W L} \leq T M X W L, T M N S L_{1} \leq T_{S L 1} \leq F W L_{1}^{-1}\left(T_{W L}\right), T M N S L_{2} \leq T_{S L 2} \leq F W L_{2}^{-1}\left(T_{W L}\right)\right\} \\
& =\left\{\left(T_{W L}, T_{S L 1}, T_{S L 2}\right): T M N W L \leq T_{W L} \leq T M X W L, T M N S L_{1} \leq T_{S L 1} \leq T M P S L_{1}\left[T M P W L^{-1}\left(T_{W L}\right)\right]\right. \\
& \left.\quad T M N S L_{2} \leq T_{S L 2} \leq T M P S L_{2}\left[T M P W L^{-1}\left(T_{W L}\right)\right]\right\}
\end{aligned}
$$

and $\mathrm{d} V$ corresponds to an increment of volume from $S$ (i.e., $\mathrm{d} T_{S L 2} \mathrm{~d} T_{S L 1} \mathrm{~d} T_{W L}$ ).

To facilitate establishing the equivalence of the representations for $p F$ in Eqs. (4.24) and (4.26), the representation in Eq. (4.26) can be rewritten as

$$
\begin{aligned}
p F= & \int_{T M N S L_{1}}^{T M X S L_{1}} \int_{-\infty}^{F S L_{21}\left(T_{S L 1}\right)} \int_{F W L_{1}\left(T_{S L 1}\right)}^{\infty} f W L\left(T_{W L}\right) f S L_{2}\left(T_{S L 2}\right) f S L_{1}\left(T_{S L 1}\right) \mathrm{d} T_{W L} \mathrm{~d} T_{S L 2} \mathrm{~d} T_{S L 1} \\
& +\int_{T M X S L_{2}}^{T M X L_{2}} \int_{-\infty}^{F S L_{12}\left(T_{S L 2}\right)} \int_{F W L_{2}\left(T_{S L 2}\right)}^{\infty} f W L\left(T_{W L}\right) f S L_{1}\left(T_{S L 1}\right) f S L_{2}\left(T_{S L 2}\right) \mathrm{d} T_{W L} \mathrm{~d} T_{S L 1} \mathrm{~d} T_{S L 2} \\
: & \int_{T M N S L_{1}}^{T M X S L_{1}} \int_{T M N S L_{2}}^{F S L_{21}\left(T_{S L 1}\right)} \int_{F W L_{1}\left(T_{S L 1}\right)}^{T M X W L} f W\left(T_{W L}\right) f S L_{2}\left(T_{S L 2}\right) f S L_{1}\left(T_{S L 1}\right) \mathrm{d} T_{W L} \mathrm{~d} T_{S L 2} \mathrm{~d} T_{S L 1} \\
& +\int_{T M N S L_{2}}^{T M X S L_{2}} \int_{T M N S L_{1}}^{F S L_{12}\left(T_{S L 2}\right)} \int_{F W L_{2}\left(T_{S L 2}\right)}^{T M X W L} f\left(T_{W L}\right) f S L_{1}\left(T_{S L 1}\right) f S L_{2}\left(T_{S L 2}\right) \mathrm{d} T_{W L} \mathrm{~d} T_{S L 1} \mathrm{~d} T_{S L 2} \\
= & \int_{S_{1}} f\left(T_{W L}, T_{S L 1}, T_{S L 2}\right) \mathrm{d} V+\int_{S_{2}} f\left(T_{W L}, T_{S L 1}, T_{S L 2}\right) \mathrm{d} V \\
= & \int_{S_{1} \cup S_{2}} f\left(T_{W L}, T_{S L 1}, T_{S L 2}\right) \mathrm{d} V,
\end{aligned}
$$

where 


$$
\begin{aligned}
\mathcal{S}_{1}= & \left\{\left(T_{W L}, T_{S L 1}, T_{S L 2}\right): F W L_{1}\left(T_{S L 1}\right) \leq T_{W L} \leq T M X W L, T M N S L_{1} \leq T_{S L 1} \leq T M X S L_{1}, T M N S L_{2} \leq T_{S L 2} \leq F S L_{21}\left(T_{S L 1}\right)\right\} \\
= & \left\{\left(T_{W L}, T_{S L 1}, T_{S L 2}\right): T M P W L\left[T M P S L_{1}^{-1}\left(T_{S L 1}\right)\right] \leq T_{W L} \leq T M X W L,\right. \\
& \left.T M N S L_{1} \leq T_{S L 1} \leq T M X S L_{1}, T M N S L_{2} \leq T_{S L 2} \leq T M P S L_{2}\left[T M P S L_{1}^{-1}\left(T_{S L 1}\right)\right]\right\}, \\
S_{2}= & \left\{\left(T_{W L}, T_{S L 1}, T_{S L 2}\right): F W L_{2}\left(T_{S L 2}\right) \leq T_{W L} \leq T M X W L, T M N S L_{1} \leq T_{S L 1} \leq F S L_{12}\left(T_{S L 2}\right), T M N S L_{2} \leq T_{S L 2} \leq T M X S L_{2}\right\} \\
= & \left\{\left(T_{W L}, T_{S L 1}, T_{S L 2}\right): T M P W L\left[T M P S L_{2}^{-1}\left(T_{S L 2}\right)\right] \leq T_{W L} \leq T M X W L,\right. \\
& \left.T M N S L_{1} \leq T_{S L 1} \leq T M P S L_{1}\left[T M P S L_{2}^{-1}\left(T_{S L 2}\right)\right], T M N S L_{2} \leq T_{S L 2} \leq T M X S L_{2}\right\},
\end{aligned}
$$

and $f\left(T_{W L}, T_{S L 1}, T_{S L 2}\right)$ and $d V$ are defined the same as in conjunction with Eq. (4.29). The approximation in Eq. (4.33) results from truncating infinite integrals at $T M N S L_{1}, T M N S L_{2}$ and $T M X W L$ as appropriate. As long as problems start and end at temperatures such that the probability of an SL failure temperature below the corresponding starting temperature (i.e., $T M N S L_{1}$ or $T M N S L_{2}$ ) is inconsequential and the probability of a WL failure temperature above TMXWL is inconsequential, the indicated approximation will have no significant effect on the calculated value for $p F$. The final equality in Eq. (4.33) is contingent on the intersection of $S_{1}$ and $S_{2}$ being a set of zero volume (i.e., measure zero) in three-dimensional space; this is established in the following two paragraphs.

The following inequalities are required by the definitions of $S_{1}$ and $S_{2}$ in Eqs. (4.34) and (4.35) for a point $\left(T_{W L}, T_{S L 1}, T_{S L 2}\right)$ in $\mathcal{S}_{1} \cap S_{2}$ :

$$
\operatorname{TMNSL}_{2} \leq T_{S L 2} \leq \operatorname{TMPSL}_{2}\left[\operatorname{TMPSL}_{1}^{-1}\left(T_{S L 1}\right)\right]
$$

and

$$
T M N S L_{1} \leq T_{S L 1} \leq T M P S L_{1}\left[\operatorname{TMPSL}_{2}^{-1}\left(T_{S L 2}\right)\right]
$$

Because $T_{M P S L_{2}^{-1}}\left(T_{S L 2}\right)$ and $T_{M P S L_{1}^{-1}}\left(T_{S L 1}\right)$ are increasing functions, the preceding inequalities imply that

$$
\begin{aligned}
& {T M P S L_{2}^{-1}}^{-1}\left(T_{S L 2}\right) \leq T M P S L_{1}^{-1}\left(T_{S L 1}\right) \\
& \operatorname{TMPSL}_{1}^{-1}\left(T_{S L 1}\right) \leq T M P S L_{2}^{-1}\left(T_{S L 2}\right),
\end{aligned}
$$

and hence that

$$
\operatorname{TMPSL}_{2}^{-1}\left(T_{S L 2}\right) \leq \operatorname{TMPSL}_{1}^{-1}\left(T_{S L 1}\right) \leq \operatorname{TMPSL}_{2}^{-1}\left(T_{S L 2}\right)
$$

In turn, the immediately preceding inequalities imply that 


$$
\operatorname{TMPSL}_{1}^{-1}\left(T_{S L 1}\right)=\operatorname{TMPSL}_{2}^{-1}\left(T_{S L 2}\right)
$$

and hence that the equalities

$$
\begin{aligned}
& T_{S L 1}=T_{M P S L_{1}}\left[\operatorname{TMPSL}_{2}^{-1}\left(T_{S L 2}\right)\right] \\
& T_{S L 2}=\operatorname{TMPSL}_{2}\left[\operatorname{TMPSL}_{1}^{-1}\left(T_{S L 1}\right)\right]
\end{aligned}
$$

hold for $\left(T_{W L}, T_{S L 1}, T_{S L 2}\right)$ in $S_{1} \cap S_{2}$. Thus, $T_{S L 1}$ and $T_{S L 2}$ fall on the line defined by the points

$$
\left[T_{S L 1}, T M P S L_{2}\left[T M P S L_{1}^{-1}\left(T_{S L 1}\right)\right]\right], T M N S L_{1} \leq T_{S L 1} \leq T M X S L_{1}
$$

or equivalently by the points

$$
\left[\operatorname{TMPSL}_{1}\left[T M P S L_{2}^{-1}\left(T_{S L 2}\right)\right], T_{S L 2}\right], T M N S L_{2} \leq T_{S L 2} \leq T M X S L_{2}
$$

Further, the relationships

$$
T M P W L\left[\operatorname{TMPSL}_{2}^{-1}\left(T_{S L 2}\right)\right]=T M P W L\left[T M P S L_{1}^{-1}\left(T_{S L 1}\right)\right] \leq T_{W L} \leq T M X W L
$$

follows from the definition of $S_{1}$ in Eq. (4.34) and the equality in Eq. (4.41).

Together, the results in Eqs. (4.44) - (4.46) imply that $S_{1} \cap S_{2}$ is the surface defined by

$$
\left[T_{W L}, T_{S L 1}, \operatorname{TMPSL}_{2}\left[\operatorname{TMPSL}_{1}^{-1}\left(T_{S L 1}\right)\right]\right]
$$

for $T M P W L\left[T M P S L_{1}^{-1}\left(T_{S L 1}\right)\right] \leq T_{W L} \leq T M X W L$ and $T M N S L_{1} \leq T_{S L 1} \leq T M X S L_{1}$, or equivalently, by

$$
\left[T_{W L}, T M P S L_{1}\left[\operatorname{TMPSL}_{2}^{-1}\left(T_{S L 2}\right)\right], T_{S L 2}\right]
$$

for $T M P W L\left[T M P S L_{2}^{-1}\left(T_{S L 2}\right)\right] \leq T_{W L} \leq T M X W L$ and $T M N S L_{2} \leq T_{S L 2} \leq T M X S L_{2}$. Thus, $S_{1} \cap S_{2}$ is a surface and the equality in Eq. (4.33) is valid.

The integrals defining $p F$ in Eqs. (4.29) and (4.33) have the same general form and differ only in the sets $S$ and $S_{1} \cup S_{2}$ being integrated over. The sets $S$ and $S_{1} \cup S_{2}$ will now be shown to be the same. As a result, the representations for $p F$ developed by Bohn (i.e., Eqs. (4.23), (4.24) and the representations developed in this presentation (i.e., Eqs. (4.25), (4.26) are the same (conditional on the approximation introduced in Eq. (4.33)). The equality of $\mathcal{S}$ and $S_{1} \cup S_{2}$ is established by showing that $S_{1} \cup S_{2} \subset S$ and that $S \subset S_{1} \cup S_{2}$. In establishing the preceding, 
the properties that $T M P S L_{1}, T M P S L_{2}$ and $T M P W L$ are nondecreasing functions of time and that $T M P S L_{1}^{-1}$, $T M P S L_{2}^{-1}$ and $T M P W L^{-1}$ are nondecreasing functions of temperature play an important role.

Let $\left(T_{W L}, T_{S L 1}, T_{S L 2}\right) \in \mathcal{S}_{1} \cup \mathcal{S}_{2}$. Then, either $\left(T_{W L}, T_{S L 1}, T_{S L 2}\right) \in \mathcal{S}_{1}$ or $\left(T_{W L}, T_{S L 1}, T_{S L 2}\right) \in \mathcal{S}_{2}$. First, assume $\left(T_{W L}, T_{S L 1}, T_{S L 2}\right) \in \mathcal{S}_{1}$. Then, the following inequalities hold:

$$
\begin{aligned}
& T M N W L=F W L_{1}\left(T M N S L_{1}\right) \leq F W L_{1}\left(T_{S L 1}\right) \leq T_{W L} \leq T M X W L \\
& T M N S L_{1} \leq T_{S L 1}=F W L_{1}^{-1}\left[F W L_{1}\left(T_{S L 1}\right)\right] \leq F W L_{1}^{-1}\left(T_{W L}\right)=T M P S L_{1}\left[T M P W L^{-1}\left(T_{W L}\right)\right] \\
& T M N S L_{2} \leq T_{S L 2} \leq F S L_{21}\left(T_{S L 1}\right) \leq F S L_{21}\left[F W L_{1}^{-1}\left(T_{W L}\right)\right]=T M P S L_{2}\left[T M P W L^{-1}\left(T_{W L}\right)\right]
\end{aligned}
$$

Thus, $\left(T_{W L}, T_{S L 1}, T_{S L 2}\right) \in S$ (see definition of $S$ in Eq. (4.32)). Similarly, if $\left(T_{W L}, T_{S L 1}, T_{S L 2}\right) \in S_{2}$, then

$$
\begin{aligned}
& T M N W L=F W L_{2}\left(T M N S L_{2}\right) \leq F W L_{2}\left(T_{S L 2}\right) \leq T_{W L} \leq T M X W L \\
& T M N S L_{1} \leq T_{S L 1} \leq F S L_{12}\left(T_{S L 2}\right) \leq F S L_{12}\left[F W L_{2}^{-1}\left(T_{W L}\right)\right]=T M P S L_{1}\left[T M P W L^{-1}\left(T_{W L}\right)\right] \\
& T M N S L_{2} \leq T_{S L 2}=F W L_{2}^{-1}\left[F W L_{2}\left(T_{S L 2}\right)\right] \leq F W L_{2}^{-1}\left(T_{W L}\right)=T M P S L_{2}\left[T M P W L^{-1}\left(T_{W L}\right)\right]
\end{aligned}
$$

which implies that $\left(T_{W L}, T_{S L 1}, T_{S L 2}\right) \in S$ (see definition of $S$ in Eq. (4.32)). Hence, $S_{1} \cup S_{2} \subset \mathcal{S}$.

Now, let $\left(T_{W L}, T_{S L 1}, T_{S L 2}\right) \in S$. Then, either

$$
T_{S L 2} \leq F S L_{21}\left(T_{S L 1}\right)=T M P S L_{2}\left[\operatorname{TMPSL}_{1}^{-1}\left(T_{S L 1}\right)\right]
$$

or

$$
\operatorname{TMPSL}_{2}\left[\operatorname{TMPSL}_{1}^{-1}\left(T_{S L 1}\right)\right]=F S L_{21}\left(T_{S L 1}\right) \leq T_{S L 2}
$$

If the inequality in Eq. (4.55) holds, then

$$
\begin{aligned}
& T M P W L\left[\operatorname{TMPSL}_{1}^{-1}\left(T_{S L 1}\right)\right]=F W L_{1}\left(T_{S L 1}\right) \leq F W L_{1}\left[F W L_{1}^{-1}\left(T_{W L}\right)\right]=T_{W L} \leq T M X W L \\
& T M N S L_{1} \leq T_{S L 1} \leq F W L_{1}^{-1}\left(T_{W L}\right) \leq F W L_{1}^{-1}(T M X W L)=T M X S L_{1}, \\
& T M N S L_{2} \leq T_{S L 2} \leq F S L_{21}\left(T_{S L 1}\right)=T M P S L_{2}\left[T M P S L_{1}^{-1}\left(T_{S L 1}\right)\right]
\end{aligned}
$$

and thus, $\left(T_{W L}, T_{S L 1}, T_{S L 2}\right) \in S_{1}$ (see definition of $S_{1}$ in Eq. (4.34)). If the inequality in Eq. (4.56) holds, then 


$$
\begin{aligned}
& T M P W L\left[T M P S L_{2}^{-1}\left(T_{S L 2}\right)\right]=F W L_{2}\left(T_{S L 2}\right) \leq F W L_{2}\left[F W L_{2}^{-1}\left(T_{W L}\right)\right]=T_{W L} \leq T M X W L \\
& T M N S L_{1} \leq T_{S L 1} \leq F S L_{21}^{-1}\left(T_{S L 2}\right)=T M P S L_{1}\left[\operatorname{TMPSL}_{2}^{-1}\left(T_{S L 2}\right)\right] \\
& T M N S L_{2} \leq T_{S L 2} \leq F W L_{2}^{-1}\left(T_{W L}\right) \leq F W L_{2}^{-1}(T M X W L)=T M X S L_{2}
\end{aligned}
$$

and thus, $\left(T_{W L}, T_{S L 1}, T_{S L 2}\right) \in \mathcal{S}_{2}$ (see definition of $S_{2}$ in Eq. (4.35)). Hence, $\left(T_{W L}, T_{S L 1}, T_{S L 2}\right) \in \mathcal{S}_{1} \cup \mathcal{S}_{2}$, and as a result, $S \subset S_{1} \cup S_{2}$.

Given that $S_{1} \cup S_{2} \subset S$ and $S \subset S_{1} \cup S_{2}$, it follows that $S=S_{1} \cup S_{2}$. Hence, the integrals in Eqs. (4.29) and (4.33) are the same. As a result, the representations for $p F$ in Eqs. (4.24) and (4.26) are the same.

Although formally correct, the preceding derivation is not very intuitive. To help facilitate an understanding of the preceding derivation, an example follows. In this example, the time-temperature curves for the WL and the two SLs are defined by

$$
\begin{aligned}
& T M P W L(t)=100+6 t \\
& \operatorname{TMPSL}_{1}(t)=100+7 t \\
& \operatorname{TMPSL}_{2}(t)=100+8 t
\end{aligned}
$$

for $0 \leq t \leq 100 \mathrm{~min}$. Then,

$$
\begin{aligned}
& T M N W L=T M N S L_{1}=T M N S L_{2}=100^{\circ} \mathrm{C} \\
& T M X W L=600^{\circ} \mathrm{C}, T M X S L_{1}=800^{\circ} \mathrm{C}, T M X S L_{2}=900^{\circ} \mathrm{C},
\end{aligned}
$$

and the set $\mathcal{S}$ defined in Eq. (4.32) corresponds to the three-dimensional region indicated in Fig. 4.1a.

The integral defining $p F$ in Eq. (4.29) corresponds to integration (i) along the $T_{W L}$ axis from $T M N W L=100^{\circ} \mathrm{C}$ to $T M X W L=600^{\circ} \mathrm{C}$ (i.e., along the line segment $\mathcal{L}_{1}$ in Fig. 4.1a), (ii) then, for each value of $T_{W L}$, along the $T_{S L 1}$ axis from $T M N S L_{1}=100^{\circ} \mathrm{C}$ to $T M P S L_{1}\left[T M P W L^{-1}\left(T_{W L}\right)\right]$, (i.e., along the line segment $\mathcal{L}_{2}$ in Fig. 4.1a), and (iii) finally, for each value of $T_{S L 1}$, along the $T_{S L 2}$ axis from $T M N S L_{2}=100^{\circ} \mathrm{C}$ to $T M P S L_{2}\left[T M P S L_{1}^{-1}\left(T_{S L 1}\right)\right]$ (i.e., along the line segment $\mathcal{L}_{3}$ in Fig. 4.1a). The line segments $\mathcal{L}_{1}, \mathcal{L}_{2}$ and $\mathcal{L}_{3}$ in Fig. 4.1a are formally defined by

$$
\begin{aligned}
\mathcal{L}_{1} & =\left\{\left[T_{W L}, \operatorname{TMPSL}_{1}(0), \operatorname{TMPSL}_{2}(0)\right]: 100 \leq T_{W L} \leq 600^{\circ} \mathrm{C}\right\} \\
& =\left\{\left[T W P W L(t), 100^{\circ} \mathrm{C}, 100^{\circ} \mathrm{C}\right]: 0 \leq t \leq 100 \mathrm{~min}\right\} \\
\mathcal{L}_{2} & =\left\{\left[T_{W L}, T_{S L 1}, T_{M P S L_{2}}(0)\right]: 100^{\circ} \mathrm{C} \leq T_{S L 1} \leq \operatorname{TMPSL}_{1}\left[\operatorname{TMPWL}^{-1}\left(T_{W L}\right)\right]\right\}
\end{aligned}
$$




$$
\begin{aligned}
& =\left\{\left[T_{W L}, T M P S L_{1}(t), 100^{\circ} \mathrm{C}\right]: 0 \min \leq t \leq T M P W L^{-1}\left(T_{W L}\right)\right\}, \\
\mathcal{L}_{3} & =\left\{\left[T_{W L}, T_{S L 1}, T_{S L 2}\right]: 100^{\circ} \mathrm{C} \leq T_{S L 2} \leq T M P S L_{2}\left[T M P W L^{-1}\left(T_{W L}\right)\right]\right\} \\
& =\left\{\left[T_{W L}, T_{S L 1}, T M P S L_{2}(t)\right]: 0 \text { min } \leq t \leq T M P S L_{2}\left[T M P W L^{-1}\left(T_{W L}\right)\right]\right\},
\end{aligned}
$$

and collectively correspond to the set $S$ (with appropriate recognition that $\mathcal{L}_{2}$ is conditional on $T_{W L}$ and that $\mathcal{L}_{3}$ is conditional on $T_{W L}$ and $T_{S L 1}$ ).

The sets $S_{1}$ and $S_{2}$ defined in Eqs. (4.34) and (4.35) correspond to the three-dimensional regions indicated in Figs. 4.1b and 4.1c, respectively. Further, $S_{I} \cap S_{2}$ corresponds to the triangular planar region that forms the top surface of $S_{1}$ in Fig. $4.1 \mathrm{~b}$ and the bottom surface of $S_{2}$ in Fig. 4.1c.

The integral over $S_{1}$ in Eq. (4.33) corresponds to integrating (i) along the $T_{S L 1}$ axis from $T M N S L_{1}=100^{\circ} \mathrm{C}$ to $T M X S L_{1}=800^{\circ} \mathrm{C}$ (i.e., along the line segment $\mathcal{L}_{4}$ in Fig. $4.1 \mathrm{~b}$ ), (ii) then, for each value of $T_{S L 1}$, along the $T_{S L 2}$ axis from $T M N S L_{2}=100^{\circ} \mathrm{C}$ to $T M P S L_{2}\left[T M P S L_{1}^{-1}\left(T_{S L 1}\right)\right]$ (i.e., along the line segment $\mathcal{L}_{5}$ in Fig. 4.1b), and (iii) finally, for each value of $T_{S L 2}$, along the $T_{W L}$ axis from $T M P W L\left[T M P S L_{1}^{-1}\left(T_{S L 1}\right)\right]$ to $T M X W L=600^{\circ} \mathrm{C}$ (i.e., along the line segments $\mathcal{L}_{6}$ in Fig. 4.1b). The line segments $\mathcal{L}_{4}, \mathcal{L}_{5}$ and $\mathcal{L}_{6}$ in Fig. $4.1 \mathrm{~b}$ are formally defined by

$$
\begin{aligned}
& \mathcal{L}_{4}=\left\{\left[\operatorname{TMPWL}(0), T_{S L 1}, \operatorname{TMPSL}_{2}(0)\right]: \quad 100 \leq T_{S L 1} \leq 800^{\circ} \mathrm{C}\right\} \\
& =\left\{\left[100^{\circ} \mathrm{C}, \operatorname{TWPSL}_{1}(t), 100^{\circ} \mathrm{C}\right]: 0 \leq t \leq 100 \mathrm{~min}\right\}, \\
& \mathcal{L}_{5}=\left\{\left[T M P W L\left[\operatorname{TMPSL}_{1}^{-1}\left(T_{S L 1}\right)\right], T_{S L 1}, T_{S L 2}\right]: \quad 100^{\circ} \mathrm{C} \leq T_{S L 2} \leq \operatorname{TMPSL}_{2}\left[\operatorname{TMPSL}_{1}^{-1}\left(T_{S L 1}\right)\right]\right\} \\
& \left.=\left\{\left[\operatorname{TMPWL}_{\operatorname{TMPSL}}^{-1}\left(T_{S L 1}\right)\right], T_{S L 1}, T M P S L_{2}(t)\right]: 0 \mathrm{~min} \leq t \leq \operatorname{TMPSL}_{1}^{-1}\left(T_{S L 1}\right)\right\}, \\
& \mathcal{L}_{6}=\left\{\left[T_{W L}, T_{S L 1}, T_{S L 2}\right]: T M P W L\left[T M P S L_{1}^{-1}\left(T_{S L 1}\right)\right] \leq T_{W L} \leq 600^{\circ} \mathrm{C}\right\} \\
& =\left\{\left[T M P W L(t), T_{S L 1}, T_{S L 2}\right]: \operatorname{TMPSL}_{1}^{-1}\left(T_{S L 1}\right) \leq t \leq 100 \mathrm{~min}\right\},
\end{aligned}
$$

and collectively correspond to the set $S_{1}$ (with appropriate recognition that $\mathcal{L}_{5}$ is conditional on $T_{S L 1}$ and that $\mathcal{L}_{6}$ is conditional on $T_{S L 1}$ and $\left.T_{S L 2}\right)$.

The integral over $S_{2}$ in Eq. (4.33) corresponds to integrating (i) along the $T_{S L 2}$ axis from $T M N S L_{2}=100^{\circ} \mathrm{C}$ to $T M X S L_{2}=900^{\circ} \mathrm{C}$ (i.e., along the line segment $\mathcal{L}_{7}$ in Fig. 4.1c), (ii) then, for each value of $T_{S L 2}$, along the $T_{S L 1}$ axis from $T M N S L_{1}=100^{\circ} \mathrm{C}$ to $T M P S L_{1}\left[T M P S L_{2}^{-1}\left(T_{S L 2}\right)\right]$ (i.e., along the line segment $\mathcal{L}_{8}$ in Fig. $4.1 \mathrm{c}$ ), and (iii) finally, for each value of $T_{S L 1}$, along the $T_{W L}$ axis from $T M P W L\left[T M P S L_{2}^{-1}\left(T_{S L 2}\right)\right]$ to $T M X W L=600^{\circ} \mathrm{C}$ (i.e., along the line segment $\mathcal{L}_{9}$ in Fig. 4.1c). The line segments $\mathcal{L}_{7}, \mathcal{L}_{8}$ and $\mathcal{L}_{9}$ in Fig. $4.1 \mathrm{c}$ are formally defined by 


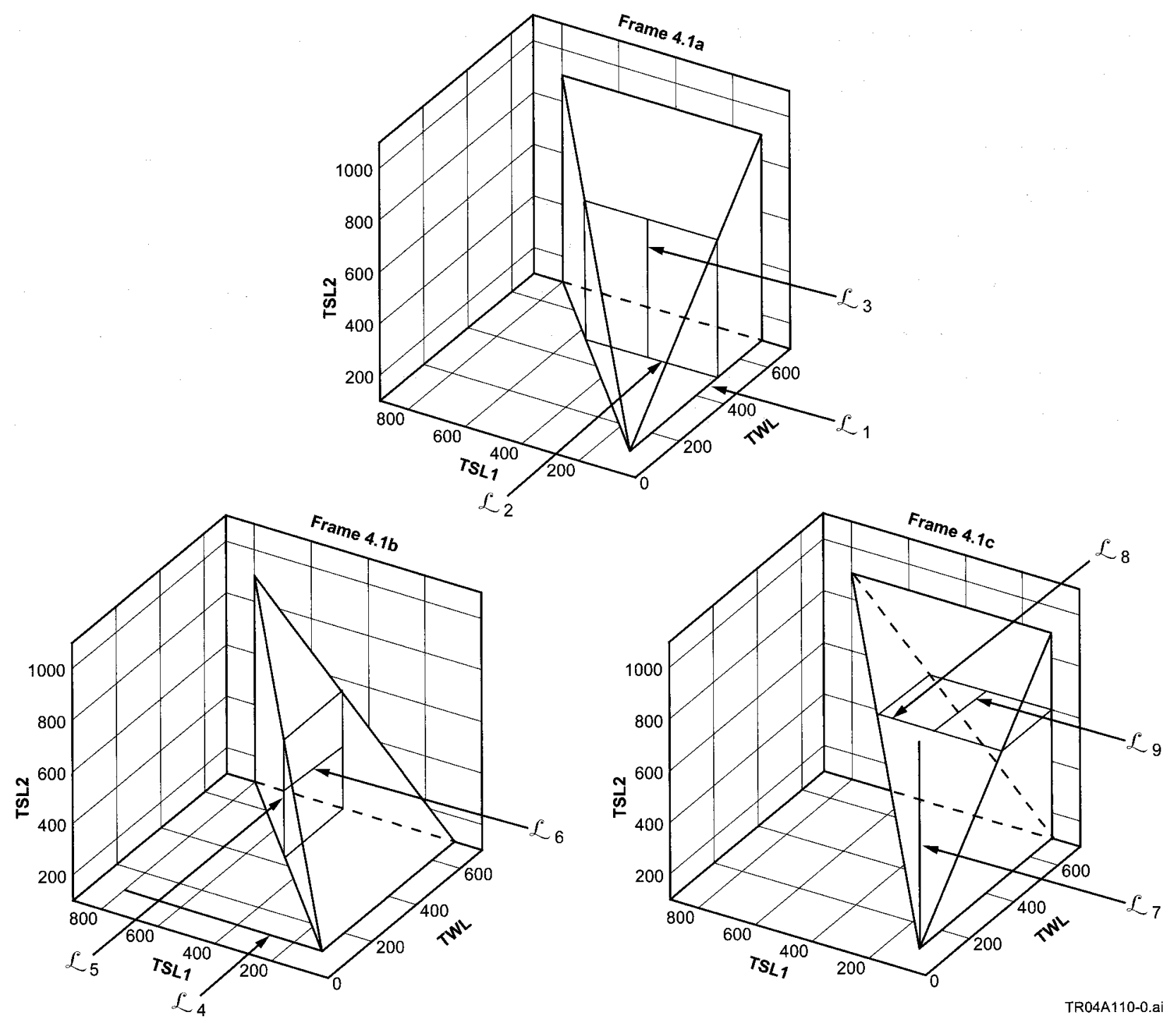

Fig. 4.1. Three-dimensional failure temperature regions used in example comparison with computational structure developed by M.P. Bohn (see App. I) for probability of loss of assured safety for system with one WL and two SLs.

$$
\begin{aligned}
\mathcal{L}_{7} & =\left\{\left[T M P W L(100), \operatorname{TMPSL}_{1}(0), T_{S L 2}\right]: 100 \leq T_{S L 2} \leq 900^{\circ} \mathrm{C}\right\} \\
& =\left\{\left[100^{\circ} \mathrm{C}, 100^{\circ} \mathrm{C}, T_{M P S L_{2}}(t)\right]: 0 \leq t \leq 100 \mathrm{~min}\right\}, \\
\mathcal{L}_{8} & =\left\{\left[T M P W L\left[T M P S L_{2}^{-1}\left(T_{S L 2}\right)\right], T_{W L 1}, T_{W L 2}\right]: 100^{\circ} \mathrm{C} \leq T_{W L 1} \leq T M P S L_{1}\left[T M P S L_{2}^{-1}\left(T_{S L 2}\right)\right]\right\} \\
& =\left\{\left[T M P W L\left[T M P S L_{2}^{-1}\left(T_{S L 2}\right)\right], T M P S L_{1}(t), T_{S L 2}\right]: 0 \min \leq t \leq T M P S L_{2}^{-1}\left(T_{S L 2}\right)\right\}, \\
\mathcal{L}_{9} & =\left\{\left[T_{W L}, T_{S L 1}, T_{S L 2}\right]: T M P W L\left[T M P S L_{2}^{-1}\left(T_{S L 2}\right)\right] \leq T_{W L} \leq 600^{\circ} \mathrm{C}\right\} \\
& =\left\{\left[T M P W L(t), T_{S L 1}, T_{S L 2}\right]: T_{\left.T M P S L_{2}^{-1}\left(T_{S L 2}\right) \leq t \leq 100 \mathrm{~min}\right\},}\right.
\end{aligned}
$$


and collectively correspond to the set $S_{2}$ (with appropriate recognition that $\mathcal{L}_{8}$ is conditional on $T_{S L 2}$ and that $\mathcal{L}_{9}$ is conditional on $T_{S L 2}$ and $T_{S L 1}$ ).

As illustrated in this example, the set $S$ integrated over in Eq. (4.29) and set $\mathcal{S}_{1} \cup \mathcal{S}_{2}$ integrated over in Eq. (4.33) are the same. Further, $S_{1} \cap S_{2}$ is a surface. Thus, the integral of the function $f$ in Eq. (4.31) over $S$ is equal to the sum of the integrals of $f$ over $S_{1}$ and $S_{2}$, respectively. Hence, the representation for $p F$ developed by Bohn for one WL and two SLs and the representation developed in this presentation are equivalent conditional on the truncation of the integrals over WL failure temperature at TMXWL in Eq. (4.33). This corresponds to truncating the integrals over WL failure temperature in the example at $600^{\circ} \mathrm{C}$. As long as the probability of WL failure below this temperature is effectively one, this truncation has no effect on $p F$ and so the two representations result in the same value for $p F$. 


\section{Multiple WLs and SLs with Individual SLS Composed of Multiple Components}

The analysis of PLOAS for a system with the following properties is now presented: multiple WLs, multiple SLs, multiple components in each SL, failure of any component in a SL constitutes failure of that SL, and failure of all SLs before failure of any WL constitutes system failure. The following topics are considered: formal mathematical representation of PLOAS (Sect. 5.1), numerical evaluation of defining integral for PLOAS with quadrature methods (Sect. 5.2), numerical evaluation of defining integral for PLOAS with Monte Carlo methods (Sect. 5.3), and example of the numerical evaluation of PLOAS (Sect. 5.4).

\subsection{Formal Representation}

As in Sects. 3 and 4, the system is assumed to involve $n W L$ WLs and $n S L$ SLs. In addition, each SL is now assumed to be composed of multiple components, with a SL failing when any one of its components fails. System failure is assumed to occur if all SLs fail before any WL fails.

The following additional notation is introduced:

$$
\begin{aligned}
n C(k) & =\text { number of components associated with SL } k \\
T M P S L_{k c}(t) & =\text { temperature }\left({ }^{\circ} \mathrm{C}\right) \text { of component } c \text { of with SL } k \text { at time } t(\min ), \\
f S L_{k c} & =\text { density function }\left({ }^{\circ} \mathrm{C}^{-1}\right) \text { for failure temperature } T_{S L} \text { of component } c \text { of SL } k .
\end{aligned}
$$

As for the WLs and SLs in prior sections, a SL component is assumed to fail if its temperature reaches its failure temperature. The WL properties $T M P W L_{j}(t)$ and $f W L_{j}$ remain as before. As for $f W L_{j}$ and $f S L_{k}$ in Sects. 3 and 4 , $f S L_{k c}$ represents aleatory uncertainty.

The objective of this section is to determine the probability $p F$ that all SLs fail before any WL fails. The sample space underlying this calculation is

$$
S=\left\{\mathbf{t}: \mathbf{t}=\left[\mathbf{t F S L}, \mathbf{t F S L}_{2}, \ldots, \mathbf{t F S L} \mathbf{L}_{n S}, t F W L_{1}, t F W L_{2}, \ldots, t F W L_{n W L}\right]\right\},
$$

where (i)

$$
\mathbf{t} \mathbf{F S} L_{k}=\left[t F S L_{k 1}, t F S L_{k 2}, \ldots, t F S L_{k, n C(k)}\right]
$$

for $k=1,2, \ldots, n S L$, (ii) $t F S L_{k c}$ is the failure time of component $c$ of SL $k$, and (iii) $t F W L_{j}$ is the failure time of WL $j$. In turn, $p F$ is defined by 


$$
p F=\operatorname{prob}(\varepsilon)
$$

where

$$
\begin{gathered}
\mathcal{E}=\left\{\mathbf{t}: \mathbf{t}=\left[\mathbf{t F S L}_{1}, \mathbf{t F S L}_{2}, \ldots, \mathbf{t F S L}_{n S L}, t F W L_{1}, t F W L_{2}, \ldots, t F W L_{n W L}\right] \in S \text { and } t F S L_{k}<t F W L_{j}\right. \\
\quad \text { for } k=1,2, \ldots, n S L, j=1,2, \ldots, n W L\}
\end{gathered}
$$

and

$$
t F S L_{k}=\min \left\{t F S L_{k 1}, t F S L_{k 2}, \ldots, t F S L_{k, n C(k)}\right\}
$$

is the failure time of SL $k$. Specifically, $\mathcal{E}$ contains the elements of $S$ for which all SLs fail before any WL fails.

The determination of $p F$ is based on the following decomposition of $\varepsilon$ :

$$
\mathcal{E}=\bigcup_{i=1}^{n T M}\left\{\bigcup_{k=1}^{n S L}\left[\bigcup_{c=1}^{n C(k)}\left(\varepsilon_{i k c} \cup \tilde{\varepsilon}_{i k c}\right)\right]\right\}
$$

where

$$
\begin{aligned}
& \varepsilon_{i k c}=\left\{\mathbf{t}: \quad \mathbf{t} \in \mathcal{E}, t_{i-1} \leq t F S L_{k c} \leq t_{i}, t_{i}<t F S L_{k d} \text { for } d \neq c, t F S L_{l}<t_{i-1} \text { for } l \neq k, t_{i}<t F W L_{j} \text { for } j=1,2, \ldots,\right. \\
& n W L\} \\
& \tilde{\varepsilon}_{i k c}=\left\{\mathbf{t}: \quad \mathbf{t} \in \mathcal{E}, t_{i-1} \leq t F S L_{k c} \leq t_{i}, t F S L_{k c} \leq t F S L_{k d} \text { for } d \neq c, t F S L_{l} \leq t F S L_{k c} \text { for } l \neq k, t F S L_{k c} \leq t F W L_{j} \text { for } j=\right.
\end{aligned}
$$

and $t M I N=t_{0}<t_{1}<\ldots<t_{n T M}=t M A X$ is a partition of $[t M I N, t M A X]$ as indicated in Fig. 2.1. The set $\tilde{\varepsilon}_{i k c}$ contains elements of $\mathcal{E}$ that have a SL $k$ component failure time $t F S L_{k d}, d \neq c$, satisfying $t F S L_{k c} \leq t F S L_{k d} \leq t_{i}$ or a SL failure time $t F S L_{l}, l \neq k$, satisfying $t_{i-1} \leq t F S L_{l} \leq t F S L_{k c}$ or a WL failure time $t F W L_{j}, j=1,2, \ldots, n W L$, satisfying $t F S L_{k c}<$ $t F W L_{j} \leq t_{i}$. The equality

$$
\operatorname{prob}(\mathcal{E})=\sum_{i=1}^{n T M} \sum_{k=1}^{n S L} \sum_{c=1}^{n C(k)} \operatorname{prob}\left(\varepsilon_{i k c}\right)+\sum_{i=1}^{n T M} \sum_{k=1}^{n S L} \sum_{c=1}^{n C(k)} \operatorname{prob}\left(\tilde{\varepsilon}_{i k c}\right)
$$

holds because the sets involved are disjoint (see parenthetical remark following Eq. (3.9)).

The equality 


$$
\lim _{n T M \rightarrow \infty} \sum_{i=1}^{n T M} \sum_{k=1}^{n S L} \sum_{c=1}^{n C(k)} \operatorname{prob}\left(\tilde{\varepsilon}_{i k c}\right)=0
$$

holds because the indicated probabilities involve products whose individual terms are proportional to $\Delta t_{i}$ (see Eq. (2.9) for an analogous situation). Further, similarly to Eqs. (3.11) and (4.6),

$$
\begin{aligned}
\operatorname{prob}\left(\varepsilon_{i k c}\right)= & \left\{\prod_{\substack{l=1 \\
l \neq k}}^{n S L} p S L_{l}\left(-\infty, t_{i-1}\right)\right\}\left\{I\left[T M P S L_{k c}\left(t_{i-1}\right), \operatorname{TMPSL}_{k c}\left(t_{i}\right), f S L_{k c}\right]\right\} \\
& \cdot\left\{\prod_{\substack{f=1 \\
f \neq c}}^{n C(k)}\left[T^{\prime} \operatorname{TMSL}_{k f}\left(t_{i}\right), \infty, f S L_{k f}\right]\right\}\left\{\prod_{j=1}^{n W L} I\left[T M P W L_{j}\left(t_{i}\right), \infty, f W L_{j}\right]\right\},
\end{aligned}
$$

where (i) the first term in the preceding product is the probability that all SLs except SL $k$ have failed by $t_{i-1}$ (i.e., $p S L_{l}\left(-\infty, t_{j-1}\right)$ is the probability that SL $l$ fails between $-\infty$ and $t_{i-1}$, and so the indicated product is the probability that all SLs except SL $k$ fail by $t_{i-1}$ ), (ii) the second term is the probability that component $c$ of SL $k$ fails in the time interval $\left[t_{i-1}, t_{i}\right]$, (iii) the third term is the probability that all components of SL $k$ except for component $c$ fail after $t_{i}$, and (iv) the fourth term is the probability that all WLs fail after $t_{i}$.

The SL failure probability $p S L_{l}\left(-\infty, t_{i-1}\right)$ in Eq. (5.11) is given by

$$
\begin{aligned}
p S L_{l}\left(-\infty, t_{i-1}\right) & =1-\prod_{d=1}^{n C(l)}\left\{1-I\left[-\infty, T M P S L_{l d}\left(t_{i-1}\right), f S L_{l d}\right]\right\} \\
& =\sum_{d=1}^{n C(l)} I\left[-\infty, \operatorname{TMPSL}_{l d}\left(t_{i-1}\right), f S L_{l d}\right] \prod_{e=d+1}^{n C(l)}\left\{1-I\left[-\infty, T M P S L_{l e}\left(t_{i-1}\right), f S L_{l e}\right]\right\} \\
& =\sum_{d=1}^{n C(l)} I\left[-\infty, \operatorname{TMPSL}_{l d}\left(t_{i-1}\right), f S L_{l d}\right] \prod_{e=d+1}^{n C(l)} I\left[T M P S L_{l e}\left(t_{i-1}\right), \infty, f S L_{l e}\right],
\end{aligned}
$$

where (i) in the first equality, $\left.I-\infty, T M P S L_{l d}\left(t_{i-1}\right), f S L_{l d}\right]$ is the probability that component $d$ of SL $l$ fails by $t_{i-1}, 1$ $-I\left[-\infty, T M P S L_{l d}\left(t_{i-1}\right), f S L_{l d}\right]$ is the probability that component $d$ of SL $l$ does not fail by $t_{i-1}$, and so the entire expression is the probability that at least one component of SL $l$ has failed by $\mathrm{t}_{i-1}$, which is the probability that SL $l$ has failed by $t_{i-1}$, (ii) in the second equality, use of the algebraic identity

$$
\prod_{d=1}^{n}\left(1-x_{d}\right)=1-\sum_{d=1}^{n} x_{d} \prod_{e=d+1}^{n}\left(1-x_{e}\right)
$$

produces the indicated expression, and (iii) in the third equality, the final expression is based on the identity 1 $I(-\infty, c, f)=I(c, \infty, f)$ for $-\infty<c<\infty$ and $f$ a density function. 
By using Eqs. (5.9) - (5.12), it follows that

$$
\begin{aligned}
& \operatorname{prob}(\varepsilon)= \lim _{n T M \rightarrow \infty} \sum_{i=1}^{n T M} \sum_{k=1}^{n S L} \sum_{c=1}^{n C(k)} \operatorname{prob}\left(\varepsilon_{i k c}\right)+\lim _{n T M \rightarrow \infty} \sum_{i=1}^{n T M} \sum_{k=1}^{n S L} \sum_{c=1}^{n C(k)} \operatorname{prob}\left(\tilde{\mathcal{E}}_{i k c}\right) \\
&= \lim _{n T M \rightarrow \infty} \sum_{i=1}^{n T M} \sum_{k=1}^{n S L} \sum_{c=1}^{n C(k)} \operatorname{prob}\left(\mathcal{E}_{i k c}\right)+0 \\
&=\lim _{n T M \rightarrow \infty} \sum_{i=1}^{n T M} \sum_{k=1}^{n S L} \sum_{c=1}^{n C(k)}\left\{\prod_{l=1}^{n S L}\left(\sum_{d=1}^{n C(l)} I\left[-\infty, T M P S L_{l d}\left(t_{i-1}\right), f S L_{l d}\right] \prod_{e=d+1}^{n C(l)} I\left[T M P S L_{l d}\left(t_{i-1}\right), \infty, f S L_{l d}\right]\right)\right\} \\
& \cdot\left\{I\left[T M P S L_{k c}\left(t_{i-1}\right), T M P S L_{k c}\left(t_{i}\right), f S L_{k c}\right]\right\}\left\{\begin{array}{l}
n C(k) \\
\left.\prod_{\substack{f=1 \\
f \neq c}}^{n+1} I\left[T M P S L_{k f}\left(t_{i}\right), \infty, f S L_{k f}\right]\right\}
\end{array}\right. \\
& \cdot\left\{\prod_{j=1}^{n W L} I\left[T M P W L_{j}\left(t_{i}\right), \infty, f W L_{j}\right]\right\},
\end{aligned}
$$

where (i) the first equality follows from Eq. (5.9), (ii) the second equality follows from Eq. (5.10), and (iii) the third equality follows from Eqs. (5.11) and (5.12). Evaluation of the final limit in Eq. (5.14) leads to the representation of $\operatorname{prob}(\mathcal{E})$, and hence $p F$, by

$$
\begin{aligned}
& p F=\sum_{k=1}^{n S L} \sum_{c=1}^{n C(k)}\left[\int_{t M I N}^{t M A X}\left\{\prod_{\substack{l=1 \\
l \neq k}}^{n S L}\left(\sum_{d=1}^{n C(l)} I\left[-\infty, T M P S L_{l d}(t), f S L_{l d}\right] \prod_{e=d+1}^{n C(l)} I\left[T M P S L_{l e}(t), \infty, f S L_{l e}\right]\right)\right\}\right. \\
& \text { - }\left\{f S L _ { k c } [ T M P S L _ { k c } ( t ) ] \left\{\left\{\prod_{\substack{f=1 \\
f \neq c}}^{n C(k)} I\left[T M P S L_{k f}(t), \infty, f S L_{k f}\right]\right\}\right.\right. \\
& \left.\cdot\left\{\prod_{j=1}^{n W L} I\left[T M P W L_{j}(t), \infty, f W L_{j}\right]\right\} \mathrm{d} T M P S L_{k c}(t)\right] \\
& =\int_{t M I N}^{t M A X}\left(\sum_{k=1}^{n S L}\left\{\prod_{\substack{l=1 \\
l \neq k}}^{n S L}\left(\sum_{d=1}^{n C(l)} I\left[-\infty, T M P S L_{l d}(t), f S L_{l d}\right] \prod_{e=d+1}^{n C(l)} I\left[T M P S L_{l e}(t), \infty, f S L_{l e}\right]\right)\right\}\right. \\
& \text { - } \left.\left\{\sum_{c=1}^{n C(k)}\left(f S L_{k c}\left[T M P S L_{k c}(t)\right]\right)\left(\prod_{\substack{f=1 \\
f \neq c}}^{n C(k)} I\left[T M P S L_{k f}(t), \infty, f S L_{k f}\right]\right)\left(\mathrm{d} T M P S L_{k c}(t) / \mathrm{d} t\right)\right\}\right) \\
& \cdot\left(\prod_{j=1}^{n W L} I\left[T M P W L_{j}(t), \infty, f W L_{j}\right]\right) \mathrm{d} t
\end{aligned}
$$


where (i) the first equality involves Riemann-Stieltjes integrals and (ii) the second equality involves the corresponding Riemann integral.

The Riemann integral defining $p F$ in Eq. (5.15) is with respect to time. As discussed in conjunction with Eqs. (2.16) and (2.17), the definition of $p F$ can be converted to an integral with respect to temperature by a suitable change of variable. This change produces

$$
\begin{aligned}
& p F=\sum_{k=1}^{n S L} \sum_{c=1}^{n C(k)} \int_{t M I N}^{t M A X}\left\{\prod_{\substack{l=1 \\
l \neq k}}^{n S L}\left(\sum_{d=1}^{n C(l)} I\left[-\infty, T M P S L_{l d}(t), f S L_{l d}\right] \prod_{e=d+1}^{n C(l)} I\left[T M P S L_{l e}(t), \infty, f S L_{l e}\right]\right)\right\} \\
& \left.\cdot\left\{\left(f S L_{k c}\left[T M P S L_{k c}(t)\right]\right)\left(\prod_{\substack{f=1 \\
f \neq c}}^{n C(k)} I T M P S L_{k f}(t), \infty, f S L_{k f}\right]\right)\left(\mathrm{d} T M P S L_{k c}(t) / \mathrm{d} t\right)\right\} \\
& \cdot\left\{\prod_{j=1}^{n W L} I\left[T M P W L_{j}(t), \infty, f W L_{j}\right]\right\} \mathrm{d} t \\
& =\sum_{k=1}^{n S L} \sum_{c=1}^{n C(k)} \int_{T M N S L_{k c}}^{T M X S L_{k c}}\left\{\prod _ { \substack { l = 1 \\
l \neq k } } ^ { n S L } \left(\sum_{d=1}^{n C(l)} I\left[-\infty, T M P S L_{l d}\left[T M P S L_{k c}^{-1}\left(T_{S L}\right)\right], f S L_{l d}\right]\right.\right. \\
& \left.\left.\cdot \prod_{e=d+1}^{n C(l)} I\left[T M P S L_{l e}\left[T M P S L_{k c}^{-1}\left(T_{S L}\right)\right], \infty, f S L_{l e}\right]\right)\right\} \\
& \cdot\left\{f S L_{k c}\left(T_{S L}\right)\right\}\left\{\prod_{\substack{f=1 \\
f \neq c}}^{n C(k)} I\left[T M P S L_{k f}\left[T M P S L_{k c}^{-1}\left(T_{S L}\right)\right], \infty, f S L_{k f}\right]\right\} \\
& \cdot\left\{\prod_{j=1}^{n W L} I\left[T M P W L_{j}\left[T M P S L_{k c}^{-1}\left(T_{S L}\right)\right], \infty, f W L_{j}\right]\right\} \mathrm{d} T_{S L} \\
& =\int_{T M N S L}^{T M X S L} G\left(T_{S L}\right) d T_{S L}
\end{aligned}
$$

where the first equality results from moving the summations in Eq. (5.15) to outside the integration, the change of variables takes place at the second equality, and the following notation is used:

$$
\begin{aligned}
& T M N S L_{k c}=T M P S L_{k c}(t M I N), c=1,2, \ldots, n C(k), k=1,2, \ldots, n S L, \\
& T M X S L_{k c}=T M P S L_{k c}(t M A X), c=1,2, \ldots, n C(k), k=1,2, \ldots, n S L, \\
& T M N S L=\min \left\{T M N S L_{k c}, c=1,2, \ldots, n C(k), k=1,2, \ldots, n S L\right\},
\end{aligned}
$$




$$
\begin{aligned}
& T M X S L= \max \left\{T M X S L_{k c}, c=1,2, \ldots, n C(k), k=1,2, \ldots, n S L\right\} \\
& G\left(T_{S L}\right)=\sum_{k=1}^{n S L} \sum_{c=1}^{n C(k)} G_{k c}\left(T_{S L}\right), \\
& G_{k c}\left(T_{S L}\right)=\left\{\prod _ { l = 1 } ^ { n S L } \left(\sum_{d=1}^{n C(l)} I\left[-\infty, T M P S L_{l d}\left[T M P S L_{k c}^{-1}\left(T_{S L}\right)\right], f S L_{l d}\right]\right.\right. \\
&\left.\left.\cdot \prod_{e=d+1}^{n C(l)} I\left(T M P S L_{l e}\left[T M P S L_{k c}^{-1}\left(T_{S L}\right)\right], \infty, f S L_{l e}\right]\right)\right\} \\
& \cdot\left\{f S L_{k c}\left(T_{S L}\right)\right\}\left\{\prod_{f=1}^{n C(k)} I\left[T M P S L_{k f}\left[T M P S L_{k c}^{-1}\left(T_{S L}\right)\right], \infty, f S L_{k f}\right]\right\} \\
& \cdot\left\{\prod_{j \neq c}^{n W L} I\left[T M P W L_{j}\left[T M P S L_{k c}^{-1}\left(T_{S L}\right)\right], \infty, f W L_{j}\right]\right\} \text { for } T M N S L_{k c} \leq T_{S L} \leq T M X S L_{k c}
\end{aligned}
$$

The simpler representation

$$
\begin{aligned}
& p F=\int_{T M N S L}^{T M X S L} \sum_{k=1}^{n S L} \sum_{c=1}^{n C(k)}\left\{\prod _ { \substack { l = 1 \\
l \neq k } } ^ { n S L } \left(\sum_{d=1}^{n C(l)} I\left[-\infty, T M P S L_{l d}\left[T M P S L_{k c}^{-1}\left(T_{S L}\right)\right], f S L_{l d}\right]\right.\right. \\
&\left.\left.\cdot \prod_{e=d+1}^{n C(l)} I\left[T M P S L_{l e}\left[T M P S L_{k c}^{-1}\left(T_{S L}\right)\right], \infty, f S L_{l e}\right]\right)\right\} \\
& \cdot\left\{f S L_{k c}\left(T_{S L}\right)\right\}\left\{\prod_{\substack{f=1 \\
f \neq c}}^{n C(k)}\left[T M P S L_{k f}\left[T M P S L_{k c}^{-1}\left(T_{S L}\right)\right], \infty, f S L_{k f}\right]\right\} \\
& \cdot\left\{\prod_{j=1}^{n W L} I\left[T M P W L_{j}\left[T M P S L_{k c}^{-1}\left(T_{S L}\right)\right], \infty, f W L_{j}\right]\right\} \mathrm{d} T_{S L},
\end{aligned}
$$

for the first integral in Eq. (5.16) results when $T M N S L_{k c}=T M N S L$ and $T M X S L_{k c}=T M X S L$ for $c=1,2, \ldots, n C(k), k$ $=1,2, \ldots, n S L$.

\subsection{Numerical Evaluation: Quadrature}

The same numerical procedures indicated in Sect. 3.2 (i.e., the quadrature formulas in Eqs. (2.49) and (2.50)) can be used to determine the failure probability $p F$ for the WL/SL configuration described in the preceding section 
(Sect. 5.1). The only difference is a change in the definition of the function $G\left(T_{S L}\right)$ resulting from the definitions for $G_{k c}\left(T_{S L}\right)$ (see Eqs. (3.19), (3.20), (5.21), (5.22)). Integrals of the form $I[c, \infty, f]$ and $I[-\infty, c, f]$ appearing in the definition of $G_{k c}\left(T_{S L}\right)$ can be approximated as indicated in Sects. 2.3 and 4.2, respectively.

\subsection{Numerical Evaluation: Monte Carlo}

The same Monte Carlo procedures indicated in Sect. 3.3 can be used to determine the failure probability $p F$ for the WL/SL configuration described in Sect. 5.1. The only real difference is in the definition of the indicator function $\delta\left(\mathbf{T}_{W L}, \mathbf{T}_{S L}\right)$ in Eq. (3.22). For the WL/SL configuration described in Sect. 5.1, $\delta$ has the more complex form $\delta\left(\mathbf{T}_{W L}, \mathbf{T}_{S L, 1}, \mathbf{T}_{S L, 2}, \ldots, \mathbf{T}_{S L, n S L}\right)$, where $\mathbf{T}_{W L}$ is the same as defined in conjunction with Eq. (3.22),

$$
\mathbf{T}_{S L, k}=\left[T_{S L, k, 1}, T_{S L, k, 2}, \ldots, T_{S L, k, n C(k)}\right]
$$

for $k=1,2, \ldots, n$, and $T_{S L, k, c}$ is the failure temperature $\left({ }^{\circ} \mathrm{C}\right)$ of component $c$ of SL $k$.

More specifically, the failure probability $p F$ for the WL/SL configuration described in Sect. 5.1 can be represented by

$$
p F=\int_{S} \delta\left(\mathbf{T}_{W L}, \mathbf{T}_{S L, 1}, \mathbf{T}_{S L, 2}, \ldots, \mathbf{T}_{S L, n S L}\right) f T(\mathbf{T}) d \mathbf{T},
$$

where

$$
\begin{aligned}
S= & \left\{\mathbf{T}: \mathbf{T}=\left[\mathbf{T}_{W L}, \mathbf{T}_{S L, 1}, \mathbf{T}_{S L, 2}, \ldots, \mathbf{T}_{S L, n S L}\right]\right. \text { consistent with the distributions that define the WL } \\
& \text { and SL component failure temperatures }\}, \\
f T(\mathbf{T})= & \begin{array}{l}
\text { density function defined on } S \text { consistent with the distributions that define the WL and SL compo- } \\
\text { nent failure temperatures }
\end{array} \\
= & \prod_{j=1}^{n W L} f W L_{j}\left(T_{W L, j}\right) \prod_{k=1}^{n S L}\left[\prod_{c=1}^{n C(k)} f S L_{k c}\left(T_{S L, k, c}\right)\right], \\
d \mathbf{T}= & \prod_{j=1}^{n W L} d T_{W L, j} \prod_{k=1}^{n S L}\left[\prod_{c=1}^{n C(k)} d T_{S L, k, c}\right], \\
t F S L_{k}= & \min \left\{T M P S L_{k c}^{-1}\left(T_{S L, k, c}\right): c=1,2, \ldots, n C(k)\right\} \text { for } k=1,2, \ldots, n S L, \text { with } T M P S L_{k c}^{-1}\left(T_{S L, k, c}\right)=-\infty \text { if } \\
T_{S L, k}= & \min \left\{T_{S L, k, c}: c=1,2, \ldots, n C(k)\right\}
\end{aligned}
$$


and

$$
\begin{aligned}
& \delta(\mathbf{T})=\delta\left(\mathbf{T}_{W L}, \mathbf{T}_{S L, 1}, \mathbf{T}_{S L, 2}, \ldots, \mathbf{T}_{S L, n S L}\right) \\
& =1 \text { if } \max \left\{t F S L_{k}: k=1,2, \ldots, n S L\right\}=-\infty \text { and } \max \left\{T_{S L, k}: k=1,2, \ldots, n S L\right\}< \\
& \min \left\{T_{W L, j}: j \in S\left(\mathbf{T}_{W L}\right)\right\} \text { with } S\left(\mathbf{T}_{W L}\right)=\left\{j: T_{W L, j}<T M P W L_{j}(t M I N)\right\} \\
& =1 \text { if }-\infty<\max \left\{t F S L_{k}: k=1,2, \ldots, n S L\right\}<\min \left\{t F W L_{j}: t F W L_{j}=T M P W L_{j}^{-1}\left(T_{W L, j}\right)\right. \text {, } \\
& T M P W L_{j}^{-1}\left(T_{W L, j}\right)=-\infty \text { if } T_{W L, j}<T M P W L_{j}(t M I N), T M P W L_{j}^{-1}\left(T_{W L, j}\right)=\infty \\
& \text { if } \left.T_{W L, j}>T M P W L_{j}(t M A X), j=1,2, \ldots, n W L\right\} \\
& =0 \text { otherwise. }
\end{aligned}
$$

With respect to the role of the indicator function $\delta(\mathbf{T})$, (i) the assignment in Eq. (5.27) picks out the T's in which all SLs fail before the start of the analysis at time $t M I N$ and any WL failing before the start of the analysis has a higher failure temperature than any SL, (ii) the assignment in Eq. (5.28) picks out the T's in which all SLs fail before the end of the analysis at $t M A X$ and also before the failure of any WL, and (iii) the assignment in Eq. (5.29) removes the probability associated with T's in which any of the WLs fail before all of the SLs from incorporation into $p F$.

The integral defining $p F$ in Eq. (5.25) can be approximated by

$$
p F \cong \sum_{i=1}^{n} \delta\left(\mathbf{T}_{i}\right) / N
$$

where

$$
\mathbf{T}_{i}=\left[\mathbf{T}_{W L, i}, \mathbf{T}_{S L, 1, i}, \mathbf{T}_{S L, 2, i}, \ldots, \mathbf{T}_{S L, n S L, i}\right]
$$

with

$$
\begin{aligned}
& \mathbf{T}_{W L, i}=\left[\mathbf{T}_{W L, 1, i}, \mathbf{T}_{W L, 2, i}, \ldots, \mathbf{T}_{W L, n W L, i}\right] \\
& \mathbf{T}_{S L, k, i}=\left[\mathbf{T}_{S L, k, 1, i}, \mathbf{T}_{S L, k, 2, i}, \ldots, \mathbf{T}_{S L, k, n C(k), i}\right], k=1,2, \ldots, n,
\end{aligned}
$$

is randomly sampled from $S$ for $i=1,2, \ldots, N$ in consistency with the joint density function $f T(\mathbf{T})$ in Eq. (5.26).

The approximation to $p F$ in Eq. (5.30) is based on simple random sampling from the joint failure temperature distribution defined by $f T(\mathbf{T})$. Importance sampling can also be used in the approximation of $p F$. In this case, the approximation to $p F$ becomes

$$
p F \cong \sum_{i=1}^{N}\left[\frac{\delta\left(\mathbf{T}_{i}\right) f T\left(\mathbf{T}_{i}\right)}{f I T\left(\mathbf{T}_{i}\right)}\right] / N,
$$


where $\mathbf{T}_{i}, i=1,2, \ldots, N$, is a random sample from $S$ generated in consistency with a joint density function

$$
f I T(\mathbf{T})=\prod_{j=1}^{n W L} f I W L_{j}\left(T_{W L, j}\right) \prod_{k=1}^{n S L}\left[\prod_{c=1}^{n C(k)} f I S L_{k c}\left(T_{S L, k, c}\right)\right]
$$

and the individual density functions appearing in the definition of $f I T(\mathbf{T})$ (i.e., $f I W L_{j}\left(T_{W L, j}\right), j=1,2, \ldots, n W L$, $\left.f I S L_{k c}\left(T_{S L, k, c}\right), c=1,2, \ldots, n C(k), k=1,2, \ldots, n S L\right)$ define importance sampling distributions for the individual WLs and SL components.

\subsection{Numerical Evaluation: Example}

The example introduced in Sect. 3.4 is modified to illustrate the WL/SL configuration described in Sect. 5.1 (Table 5.1). Specifically, a system involving two WLs (i.e., $n W L=2$ ), two SLs (i.e., $n S L=2$ ), and two components in each SL (i.e., $n C(1)=n C(2)=2$ ) is considered, with (i) the WL temperature curves $T M P W L_{j}(t)$ the same as in Eq. (3.30), (ii) the component temperature curves $T M P S L_{k c}(t), c=1,2$, for SL $k$ the same as the corresponding SL temperature curve $T M P S L_{k}(t)$ in Eq. (3.31) (i.e., $T M P S L_{k c}(t)=T M P S L_{k}(t)$ ), (iii) the WL failure temperature density functions $f W L_{j}\left(T_{W L}\right)$ the same as in Sect. 3.4, and (iv) the component failure temperature density functions $f S L_{k c}\left(T_{S L}\right), c=1,2$, for SL $k$ the same as the corresponding SL failure temperature density function $f S L_{k}\left(T_{S L}\right)$ in Sect. 3.4 (i.e., $f S L_{k c}\left(T_{S L}\right)=f S L_{k}\left(T_{S L}\right)$ ). As in Sect. 3.4, the WL failure temperatures are assumed to be independent although the distributions defined by $f W L_{I}\left(T_{W L}\right)$ and $f W L_{2}\left(T_{W L}\right)$ are the same; the same assumption is also made for the four SL component failure temperature distributions.

The system with internal SL components illustrated in this section has a higher failure probability than the corresponding system in Sect. 4.4 with no internal SL components (i.e., $p F \cong 4.55 \times 10^{-6}$ in Table 5.1 versus $p F \cong 1.56$ $\times 10^{-6}$ in Table 4.1). This difference results because a SL is assumed to fail when either of its components fails. Thus, all other things being the same (which is indeed the case for the examples in Sects. 4.4 and 5.4), a SL with two components is more likely to fail by a given time than a SL with only one component, which is effectively the situation considered in Sect.4.4. In turn, this failure pattern and the assumption that the WLs in the two examples have the same properties leads to the indicated inequality involving the failure probability $p F$. 
Table 5.1. Approximation of Failure Probability $p F$ for System Defined in Sect. 5.4 with Two WLs, Two SLs, Two Components in Each SL, Normal Distributions for WL and SL Component Failure Temperatures, Failure of Either Component in a SL Constituting Failure of That SL, and Failure of Both SLs before Either WL Constituting System Failure (i.e., the Failure Configuration Described in Sect. 5.1)

\begin{tabular}{|c|c|c|c|c|c|c|}
\hline & $\mathrm{N}^{\mathrm{b}}$ & $\begin{array}{l}\text { Trapezoidal } \\
\text { Rule }\end{array}$ & $\begin{array}{l}\text { Simpson's } \\
\text { Rule }^{d}\end{array}$ & $\mathrm{~N}^{\mathrm{e}}$ & $\begin{array}{l}\text { Random } \\
\text { Sampling }\end{array}$ & $\begin{array}{l}\text { Importance } \\
\text { Samplingg }\end{array}$ \\
\hline & 3 & $3.230 \mathrm{E}-07$ & $4.306 \mathrm{E}-07$ & 1,000 & $0.000 \mathrm{E}+00$ & $8.163 \mathrm{E}-14$ \\
\hline & 5 & $1.615 \mathrm{E}-07$ & $1.077 \mathrm{E}-07$ & 10,000 & $0.000 \mathrm{E}+00$ & $1.064 \mathrm{E}-10$ \\
\hline & 9 & $2.629 \mathrm{E}-07$ & $2.967 \mathrm{E}-07$ & 100,000 & $0.000 \mathrm{E}+00$ & $1.374 \mathrm{E}-08$ \\
\hline & 17 & $1.356 \mathrm{E}-07$ & $9.316 \mathrm{E}-08$ & $1,000,000$ & $4.000 \mathrm{E}-06$ & $1.416 \mathrm{E}-06$ \\
\hline & 33 & $6.111 \mathrm{E}-06$ & $8.102 \mathrm{E}-06$ & $10,000,000$ & $4.700 \mathrm{E}-06$ & $3.451 \mathrm{E}-06$ \\
\hline & 65 & $4.576 \mathrm{E}-06$ & 4.064E-06 & $100,000,000$ & $4.700 \mathrm{E}-06$ & $4.468 \mathrm{E}-06$ \\
\hline & 129 & $4.552 \mathrm{E}-06$ & $4.545 \mathrm{E}-06$ & & & \\
\hline & 257 & $4.552 \mathrm{E}-06$ & $4.552 \mathrm{E}-06$ & & & \\
\hline & 513 & ---- & $4.553 \mathrm{E}-06$ & & & \\
\hline & 1025 & ---- & $4.553 \mathrm{E}-06$ & & & \\
\hline & 2049 & ---- & $4.553 \mathrm{E}-06$ & & & \\
\hline \multicolumn{7}{|c|}{ Calculations performed with CPLOAS program (App. III). } \\
\hline \multicolumn{7}{|c|}{$\begin{array}{l}\text { Number of evaluations of } G\left(T_{S L}\right) \text { (see Eq. (5.16)) with trapezoidal rule and Simpson's rule, which corresponds to the interval [TMNSL, } \\
T M X S L] \text { being divided into } N-1 \text { subintervals. }\end{array}$} \\
\hline \multicolumn{7}{|c|}{ Approximation to $p F$ obtained with trapezoidal rule (see Eq. (2.49)). } \\
\hline & \multicolumn{6}{|c|}{ Approximation to $p F$ obtained with Simpson's rule (see Eq. $(2.50)$ ). } \\
\hline \multicolumn{7}{|c|}{$\begin{array}{l}\text { Number of evaluation of } \delta(\mathbf{T})=\delta\left(\mathbf{T}_{W L}, \mathbf{T}_{S L, 1,}, \mathbf{T}_{S L, 2)}\right)=\delta\left(\mathbf{T}_{W L, 1} \mathbf{T}_{W L, 2,}, \mathbf{T}_{S L, 1,1}, \mathbf{T}_{S L, 1,2}, \mathbf{T}_{S L, 2,1}, \mathbf{T}_{S L, 2,2}\right) \text { (see Eqs. (5.27) - (5.29)) for random sampling } \\
\text { and importance sampling. }\end{array}$} \\
\hline \multicolumn{7}{|c|}{ Approximation to $p F$ obtained with random sampling (see Eq. $(5.30)$ ). } \\
\hline g & \multicolumn{6}{|c|}{$\begin{array}{l}\text { Approximation to } p F \text { obtained with importance sampling with uniform distributions for } T_{W L, 1}, T_{W L, 2}, T_{S L, 1,1}, T_{S L, 2,2}, T_{S L, 2,1}, T_{S L, 2,2} \text { (see Eqs. } \\
(5.31) \text { and (5.32), with } f W L_{j}\left(T_{W L, j}\right) \text { and } f I S L_{k c}\left(T_{S L, k c}\right) \text { defined as indicated in Eqs. (2.70) and (2.71), respectively). }\end{array}$} \\
\hline
\end{tabular}




\section{Example Uncertainty and Sensitivity Analysis}

Substantial epistemic (i.e., state of knowledge) uncertainties ${ }^{8-11}$ are present in the analysis of WL/SL systems of the form described in this presentation. In particular, epistemic uncertainty is present in both the modeling of the temperature responses of the system and in the definition of the distributions that characterize the variability in WL and SL failure temperatures. In practice, these uncertainties can be both large and poorly characterized. As a result, a properly designed and implemented uncertainty and sensitivity analysis is an important part of a reliability analysis of a WL/SL system or any other complex system. ${ }^{31-35}$ In this section, two approaches to uncertainty and sensitivity analysis of the reliability of a WL/SL system are illustrated for a hypothetical WL/SL system involving 2 WLs and 2 SLs. The first approach is a traditional uncertainty analysis based on the use of probability to characterize epistemic uncertainty. ${ }^{36-43}$ The second approach uses evidence theory to characterize epistemic uncertainty. ${ }^{44-51}$

Uncertainty analysis involves the investigation of a relationship of the form

$$
y=f(\mathbf{x}), \mathbf{x}=\left[x_{1}, x_{2}, \ldots, x_{n X}\right]
$$

where $\mathbf{x}$ is a vector of imprecisely known analysis inputs and $f$ is a function (i.e., a model) that produces an analysis result $y$ for each possible value for $\mathbf{x}$. In practice, $f$ can be quite complicated and might correspond to the numerical solution of a nonlinear partial differential equation or possibly to the operation of several successively linked models as is often the case in performance assessments for complex systems. For convenience, $y$ is represented as being real (i.e., scalar) valued, although in most analyses $y$ is a vector of high dimension. In the example that follows, $y$ corresponds to the probability $p F$ that the WLs fail to deactivate a system composed of 2 WLs and 2 SLs in a fire environment, and $f$ corresponds to the calculational model for $p F$ described in Sect. 4 . Uncertainty in $\mathbf{x}$ results in many possible values for $y$ of varying levels of credence. The goal of uncertainty analysis is to provide a formal representation of the uncertainty in $y$ that derives from the uncertainty in $\mathbf{x}$.

The following topics are considered: representation of uncertainty with probability theory (Sect.6.1), representation of uncertainty with evidence theory (Sect. 6.2), numerical estimation complementary cumulative distribution, belief and plausibility functions (Sect. 6.3), an example problem for the illustration of uncertainty and sensitivity analysis (Sect. 6.4), an example uncertainty and sensitivity analysis (Sect. 6.5), and formal justification of a procedure used to construct uncertainty representations in the context of evidence theory (Sect. 6.6).

\subsection{Representation of Uncertainty with Probability Theory}

The traditional approach to uncertainty analysis is to use probability to characterize the uncertainty in the elements $x_{i}, i=1,2, \ldots, n X$, of $\mathbf{x}$ and then to determine the resultant probability distribution for $y$. With this approach, distributions 


$$
D_{1}, D_{2}, \ldots, D_{n X}
$$

are defined to characterize the uncertainty in $x_{1}, x_{2}, \ldots, x_{n X}$. Specifically, $D_{i}$ is a probability distribution that provides a mathematical characterization of the uncertainty in $x_{i}$ in the sense that $D_{i}$ is providing a degree of belief representation with respect to where the appropriate value of $x_{i}$ to use in the determination of $y$ is located. Various correlations and other restrictions may also accompany the definition of $D_{1}, D_{2}, \ldots, D_{n X}$. In practice, the $D_{i}$ are often determined through expert review processes. ${ }^{52-63}$ Collectively, all possible values for $\mathbf{x}$ constitute a set $\mathrm{e} X$, with $X$ often referred to as the sample space or the universal set for the $\mathbf{X}$ 's. When viewed formally, the distributions in Eq. (6.2) define a probability space $(\mathrm{e} X, \mathbb{X}, p X)$, where $\mathrm{e} X$ is the sample space, $\mathrm{e} X$ is the set of events, and $p_{X}$ is the probability measure that assigns probabilities to elements of $\mathbb{X}$ (Ref. 64, Sect. IV.4); similarly, each individual distribution $D_{i}$ in Eq. (6.2) defines a probability space $\left({ }^{\circ} X_{i}, \mathbb{X}_{i}, p_{i}\right)$ for $x_{i}$. For notational purposes, it is convenient to represent the distribution of $\mathbf{x}$ over $X$ defined by the individual distributions $D_{1}, D_{2}, \ldots, D_{n X}$ and any additional correlations and/or restrictions by a density function $d_{X}(\mathbf{x})$ defined on $X$

Once the uncertainty in $\mathbf{x}$ is characterized in a probabilistic format as indicated in the preceding paragraph, the corresponding uncertainty in $y=f(\mathbf{x})$ can also be characterized in a probabilistic format. Specifically, the probability $\operatorname{prob}(y>v)$ that a value $y$ larger than $v$ could result from the uncertainty in $\mathbf{x}$ is given by

$$
\operatorname{prob}(y>v)=\int_{\odot} \delta_{v}[y(\mathbf{x})] d_{X}(\mathbf{x}) \mathrm{d} X
$$

where

$$
\delta_{v}[y(\mathbf{x})]= \begin{cases}1 & \text { if } y(\mathbf{x})>v \\ 0 & \text { otherwise }\end{cases}
$$

In effect, $\operatorname{prob}(y>v)$ defines the complementary cumulative distribution function (CCDF) for $y$ that derives from the uncertainty in $\mathbf{x}$ characterized by the distribution $D_{1}, D_{2}, \ldots, D_{n X}$. Complementary cumulative distribution functions are a standard format for the representation of uncertainty because they answer the question "How likely is $y$ to be this large or larger?", which is usually the question of interest in risk and uncertainty assessments.

\subsection{Representation of Uncertainty with Evidence Theory}

The evidence theory approach to the representation of the uncertainty associated with the elements $x_{i}, i=1,2$, $\ldots, n X$, of $\mathbf{x}$ is to assign an uncertainty structure to each $x_{i}$ based on what are called basic probability assignments (BPAs). Specifically, (i) the set $X_{i}$ of all possible values for $x_{i}$ is identified, (ii) a finite collection $\mathbb{X}_{i}=\left\{\odot X_{i j}, j=1\right.$, $2, \ldots, n X(i)\}$ of subsets of $X_{i}$ is identified about which uncertainty information is available, and (iii) a set function $m_{i}$ is defined for subsets $\mathscr{W}$ of $X_{i}$ such that 


$$
m_{i}(U)= \begin{cases}p_{i j}>0 & \text { if } \mathscr{U}=X_{i j} \in \mathbb{X}_{i} \\ 0 & \text { otherwise }\end{cases}
$$

and

$$
\sum_{j=1}^{n X(i)} m_{i}\left(\cdot X_{i j}\right)=1
$$

The function $m_{i}(\mathscr{U})$ defines the BPA for the subset $\mathscr{U}$ of $X_{i}$, with the BPA for $U$ corresponding to the amount of probability that can be assigned to $\mathscr{U}$ but for which there is no information or rationale to assign this probability to

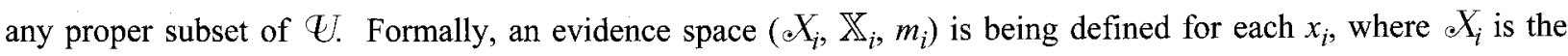
sample space or universal set (i.e., the set of all possible values for $x_{i}$ ), $\mathbb{X}_{i}$ is the set of all subsets of $X_{i}$ with nonzero BPAs, and $m_{i}$ is the function such that, if $\mathscr{U} \subset \propto X_{i}$, then $m_{i}(\mathcal{U})$ is the BPA for $\mathscr{U}$.

Similarly to probability theory, the evidence theory representation $\left(\mathrm{e} X, \mathbb{X}, m_{X}\right)$ for the uncertainty in $\mathbf{x}$ is built up from the representations for the uncertainty in the elements $x_{i}$ of $\mathbf{X}$. With the simplifying assumption that there

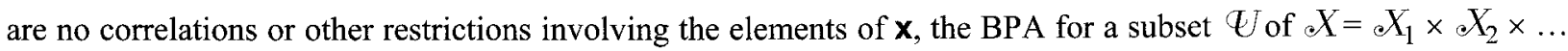
$\times X_{n X}$ is given by

$$
m_{X}(\mathscr{U})= \begin{cases}\prod_{i=1}^{n X} m_{i}\left(\mathcal{U}_{i}\right) & \text { if } \mathscr{U}=\mathscr{U}_{1} \times \mathcal{U}_{2} \times \ldots \times \mathcal{U}_{n X} \text { and } \mathcal{U}_{i} \in \mathbb{X}_{i} \text { for } i=1,2, \ldots, n X \\ 0 & \text { otherwise. }\end{cases}
$$

Further, $\mathbb{X}$ contains the subsets $\mathscr{U}$ of $\triangle$ for which $m_{X}(\mathscr{U})>0$. In turn, the evidence space $\left(\propto X \mathbb{X}, m_{X}\right)$ can be used to develop an evidence theory representation $\left(Y, \mathbb{Y}, m_{Y}\right)$ for the uncertainty in $y=f(\mathbf{x})$. However, before this is done, it is useful to introduce the constructions used to represent uncertainty in conjunction with evidence theory.

Unlike probability theory which uses probability as the only representation for uncertainty, evidence theory uses two representations for uncertainty. These representations are referred to as belief and plausibility. For a subset $U$ of a universal set $\odot$ on which a function $m(V)$ defining BPAs for subsets $V$ of $\triangle$ has been defined, the belief $\operatorname{Bel}(U)$ and plausibility $P l(U)$ of $U$ are defined by

$$
\operatorname{Bel}(\mathcal{U})=\sum_{V \in \mathcal{U}} m(V)
$$

and

$$
P l(\mathcal{U})=\sum_{V \cap \mathcal{U} \neq \phi} m(\mathcal{V})
$$

Conceptually, $\operatorname{Bel}(\mathscr{U})$ is the smallest amount of probability that must be assigned to $\mathscr{U}$, and $\operatorname{Pl}(\mathscr{U})$ is the largest amount of probability that could possibly be assigned to $U$. Put another way, $\operatorname{Bel}(\mathcal{U})$ is the smallest probability 
that can be assigned to $U$ without violating the constraints imposed by the BPA assignments given by $m$, and $P l(U)$ is the largest probability that can be assigned to $U$ without violating the constraints imposed by the BPA assignments given by $m$.

In probability theory, the probability of a set and its complement are related by

$$
\operatorname{prob}(U)+\operatorname{prob}\left(\Psi^{c}\right)=1
$$

where (i) $U$ is a subset of the sample space under consideration (subject to the usual constraints with respect to $U$ belonging to the $\sigma$-algebra of subsets of the sample space associated with a conceptually complete development of probability) and (ii) $\mathscr{U}^{c}$ is the complement of the set $\mathscr{U}$. Thus, the specification of the probability of a set also determines the probability of its complement. Less restrictive constraints hold for belief and plausibility. In particular,

$$
\begin{aligned}
& \operatorname{Bel}(\mathscr{U})+\operatorname{Bel}\left(\Psi^{c}\right) \leq 1 \\
& \operatorname{Pl}(U)+\operatorname{Pl}\left(\Psi^{c}\right) \geq 1 \\
& \operatorname{Bel}(U)+\operatorname{Pl}\left(\Psi^{c}\right)=1 .
\end{aligned}
$$

As a result, the use of belief and plausibility allows finer gradations in the expression of uncertainty than is the case with the use of probability in that an expression of a lack of knowledge about $\mathscr{U}$ does not necessarily imply knowledge about $\Psi^{c}$.

For notational convenience, let $Y$ denote the set $\{y: y=f(\mathbf{x}), \mathbf{x} \in \mathrm{e} X\}$. In concept, BPAs could be developed for the subsets of $\zeta$. In practice, the direct consideration of beliefs and plausibilities for subsets of $Y$ leads to more readily computed results. Specifically, the belief $B e l_{Y}(\mathcal{U})$ and plausibility $P l_{Y}(\mathcal{U})$ of a subset $\mathcal{U}$ of $Y$ are given by

$$
\operatorname{Bel}_{Y}(U)=\operatorname{Bel}_{X}\left[f^{-1}(U)\right]=\sum_{\mathcal{D} \subset f^{-1}(U)} m_{X}(\mathcal{V})
$$

and

$$
P l_{Y}(U)=P l_{X}\left[f^{-1}(\mathcal{U})\right]=\sum_{\mathcal{V} \cap f^{-1}(\mathcal{U}) \neq \phi} m_{X}(\mathcal{D})
$$

where $B e l_{X}$ and $P l_{X}$ correspond to the set functions defining belief and plausibility for subsets of $\mathrm{C}$ on the basis of the set function $m_{X}$ defined in Eq. (6.7) and $f^{-1}$ denotes the inverse of $f\left(\right.$ i.e., $f^{-1}(\mathcal{U})=\{\mathbf{x}: \mathbf{x} \in X$ and $f(\mathbf{x}) \in \mathscr{U}\}$. 
Just as CCDFs provide the standard representational format for uncertainty in the context of probability theory, complementary cumulative belief functions (CCBFs) and complementary cumulative plausibility functions (CCPFs) provide standard representational formats for uncertainty in the context of evidence theory. Specifically, the CCBF and the CCPF associated with the set $Y$ are defined by

$$
\operatorname{Bel}_{Y}(y>v)=\operatorname{Bel}_{X}\left(f^{-1}\left(Y_{v}\right)\right)=\sum_{V \subset f^{-1}\left(J_{v}\right)} m_{X}(v)
$$

and

$$
P l_{Y}(y>v)=P l_{X}\left(f^{-1}\left(Y_{v}\right)\right)=\sum_{\mathcal{D} \cap f^{-1}\left(Y_{v}\right) \neq \phi} m_{X}(v),
$$

where $\bigvee_{v}=\{y: y>v, y \in D\}$ and $B e l_{Y}(y>v)$ and $P l_{Y}(y>v)$ designate the belief and plausibility, respectively, that a value of $y$ larger than $v$ could occur (i.e., a value in the set $Y_{v}$ ).

The sets

$$
\begin{aligned}
& \mathcal{C D} \mathscr{F}=\{[v, \operatorname{prob}(y>v)]: v \in D\} \\
& C C P \mathscr{F}=\left\{\left[v, P l_{Y}(y>v)\right]: v \in D\right\} \\
& C C B \mathscr{F}=\left\{\left[v, \operatorname{Bel}_{Y}(y>v)\right]: v \in \mathcal{Y}\right\}
\end{aligned}
$$

provide formal representations for the CCDF, CCPF and CCBF associated with $y$.

\subsection{Numerical Estimation of CCDFs, CCBFs and CCPFs}

Numerical estimation of CCDFs, CCBFs and CCPFs is now considered. In practice, the CCDF for $y$ is not obtained by a traditional numerical evaluation of integrals of the form appearing in Eq. (6.3). Rather, a Monte Carlo procedure is used. With such a procedure, a random or Latin hypercube sample $\mathbf{x}_{k}, k=1,2, \ldots, n S$, is generated from $X$ in consistency with the distributions $D_{1}, D_{2}, \ldots, D_{n X}$ for the elements of $\mathbf{x}$ and any associated restrictions. ${ }^{65,66}$ Then, prob $(y>v)$ is approximated by

$$
\operatorname{prob}(y>v) \cong \sum_{k=1}^{n S} \delta_{v}\left[y\left(\mathbf{x}_{k}\right)\right] / n S
$$

where the indicator function $\delta_{v}$ is defined in Eq. (6.4). 
A similar Monte Carlo procedure can also be used to estimate CCBFs and CCPFs. ${ }^{51,67,68}$ Again, a random or Latin hypercube sample $\mathbf{x}_{k}, k=1,2, \ldots, n S$, is generated from $\mathrm{X}$ in consistency with distributions for the $x_{i}$ that will provide a dense sampling coverage of $\mathrm{e} X$ as the sample size increases. The selected sampling distributions can affect the rate at which the resultant approximations converge to the corresponding beliefs and plausibilities but do not affect the limiting values except in special cases where sets of measure zero have nonzero BPAs. Once the indicated sample is generated, the exceedance plausibilities that define the CCPF can be estimated by

$$
P l_{Y}(y>v) \cong P l_{X}\left(\cdot X_{v}\right)=\sum_{D \cap X_{v} \neq \phi} m_{X}(\mathcal{V})
$$

where $X_{v}=\left\{\mathbf{x}_{k}: y_{k}=f\left(\mathbf{x}_{k}\right)>v\right\}$. The exceedance beliefs that define the CCBF cannot be estimated directly because the subset relation used in the definition belief (see Eq. (6.8)) cannot be employed when the sets with nonzero BPAs have infinite numbers of elements and a finite sample is in use. Rather, the relationship between belief and plausibility indicated in Eq. (6.13) must be used to convert from a problem involving the estimation of belief to a problem involving the estimation of plausibility. Specifically,

$$
\begin{aligned}
\operatorname{Bel}_{Y}(y>v) & \cong \operatorname{Bel}_{X}\left(e X_{v}\right) \\
& =1-P l_{X}\left(e X_{v}^{c}\right) \\
& =1-\sum_{\mathcal{D} \cap X_{v}^{c} \neq \phi} m(\mathcal{D}),
\end{aligned}
$$

where $X_{v}$ is defined in conjunction with Eq. (6.22).

\subsection{Problem for Analysis}

The example uncertainty analyses are based on the hypothetical system of 2 WLs and 2 SLs described in Eqs. (3.30) - (3.31). The sixteen variables used to characterize this system are treated as being uncertain (Table 6.1). Each variable has an uncertainty range $[a, b]$ as indicated in Table 6.1. As this example is for illustration of ideas, it is assumed for simplicity that the uncertainty in each variable's possible values is specified in the same manner by four independent experts (Table 6.2, Fig. 6.1). This is unlikely to be the case in a real analysis but providing different uncertainty specifications for each variable would complicate the presentation of this example while adding little to its illustrative value. The information indicated in Table 6.2 is encoded into a probability distribution for each variable in Table 6.1 for use in a probabilistic representation of the uncertainty in $p F$ and into BPAs for use in an evidence theory representation of the uncertainty in $p F$. 
Table 6.1. Uncertain Variables and Associated Uncertainty Ranges Considered in Example Uncertainty Analyses

$c_{1}-$ Temperature $\left({ }^{\circ} \mathrm{C}\right)$ of WLs and SLs before start of fire. Range: $\left[-30,40^{\circ} \mathrm{C}\right]$.

$c_{2}$ - Temperature increase $\left({ }^{\circ} \mathrm{C}\right)$ above $c_{1}$ at steady state. Range: $\left[800,1000^{\circ} \mathrm{C}\right]$.

$c_{31}$ - Peak amplitude of temperature transient for WL 1. Range: $\left[-2600,-100^{\circ} \mathrm{C}\right]$.

$c_{32}$ - Peak amplitude of temperature transient for WL 2. Range: $\left[-2600,-100^{\circ} \mathrm{C}\right]$.

$c_{41}$ - Thermal heating time constant $\left(\mathrm{min}^{-1}\right)$ for WL 1 . Range: [0.2, $\left.0.4 \mathrm{~min}^{-1}\right]$.

$c_{42}$ - Thermal heating time constant $\left(\min ^{-1}\right)$ for WL 2. Range: [0.2, $\left.0.4 \mathrm{~min}^{-1}\right]$.

$c_{51}$ - Frequency response $\left(\mathrm{min}^{-1}\right)$ of temperature transient for WL 1. Range: [0.1, $\left.0.2 \mathrm{~min}^{-1}\right]$.

$c_{52}$ - Frequency response $\left(\mathrm{min}^{-1}\right)$ of temperature transient for WL 2. Range: [0.1, $\left.0.2 \mathrm{~min}^{-1}\right]$.

$c_{61}-$ Time constant $\left(\mathrm{min}^{-1}\right)$ determining the rate at which WL 1 reaches steady state temperature. Range: [0.02, $\left.0.04 \mathrm{~min}^{-1}\right]$.

$c_{62}-$ Time constant $\left(\mathrm{min}^{-1}\right)$ determining the rate at which WL 2 reaches steady state temperature. Range: [0.02, $0.04 \mathrm{~min}^{-1}$ ].

$c_{71}$ - Factor (dimensionless) used to account for more rapid heating in SL 1 than in the associated WL (i.e., WL

2). Range: $[0.5,0.8]$.

$c_{72}$ - Factor (dimensionless) used to account for more rapid heating in SL 2 than in the associated WL (i.e., WL

2). Range: $[0.5,0.8]$.

$c_{8}$ - Expected value $\left({ }^{\circ} \mathrm{C}\right)$ of normal distribution for WL failure temperatures. Range: $\left[255,285^{\circ} \mathrm{C}\right]$.

$c_{9}-$ Standard deviation $\left({ }^{\circ} \mathrm{C}\right)$ of normal distribution for WL failure temperatures. Range: $\left[4,12^{\circ} \mathrm{C}\right]$.

$c_{10}$ - Expected value $\left({ }^{\circ} \mathrm{C}\right)$ of normal distribution for SL failure temperature. Range: $\left[590,610^{\circ} \mathrm{C}\right]$.

$c_{11}$ - Standard deviation $\left({ }^{\circ} \mathrm{C}\right)$ of normal distribution for SL failure temperature. Range: $\left[15,22^{\circ} \mathrm{C}\right]$.

The construction of a probability distribution from the information in Table 6.2 is considered first. Expert 1 only specifies an interval. The usual probabilistic encoding of this type of sparse information is to assume a uniform distribution over the specified interval [Ref. 69, pp. 52-62]. That is, Expert 1 is assumed to have specified a probability distribution with a density function given by

$$
d_{1}(v)= \begin{cases}1 /(b-a) & \text { if } a \leq v \leq b \\ 0 & \text { otherwise }\end{cases}
$$

Experts 2 and 3 have in essence specified quantiles on CDFs. Again, in consistency with common procedure, uniform distributions are assumed for the variable between these quantiles. As a result, the density functions associated with Experts 2 and 3 have the form 
Table 6.2. Illustrative Specification of Uncertainty Information Used in Example Uncertainty Analyses with Probability Theory and Evidence Theory for Variables in Table 6.1 (see Fig. 6.1 for a graphical representation of the indicated uncertainty specifications)

Expert 1: States appropriate value for variable is in the interval $I_{11}=[a, b]$ but provides no information on uncertainty structure within $[a, b]$.

Expert 2: Divides $[a, b]$ into five nonoverlapping intervals of equal length (i.e., $I_{2 i}=[a+(b-a)(i-1) / 5, a+(b-$ a) $i / 5)$ for $i=1,2,3,4$ and $I_{25}=[a+(b-a)(i-1) / 5, a+(b-a) i / 5]$ for $\left.i=5\right)$ and states that the appropriate value for the variable is equally likely to be in each of these intervals.

Expert 3: Divides $[a, b]$ into following five nonoverlapping intervals: $I_{31}=[a, a+(b-a) / 10], I_{32}=[a+(b-$ $a) / 10, a+4(b-a) / 10), I_{33}=[a+4(b-a) / 10, a+6(b-a) / 10), I_{34}=[a+6(b-a) / 10, a+9(b-a) / 10), I_{35}=[a+$ $9(b-a) / 10, b]$. States that the probability (i.e., likelihood) that the appropriate value for the variable is contained in each of these intervals is $0.05,0.2,0.5,0.2$ and 0.05 , respectively.

Expert 4: Divides $[a, b]$ into following five nested intervals: $I_{41}=[a+4(b-a) / 10, a+6(b-a) / 10), I_{42}=[a+$ $3(b-a) / 10, a+7(b-a) / 10), I_{43}=[a+2(b-a) / 10, a+8(b-a) / 10), I_{44}=[a+(b-a) / 10, a+9(b-a) / 10), I_{45}=$ $[a, b]$. States that amount of probability (i.e., likelihood) that can be assigned to the proposition that a given interval contains the appropriate value to use for the variable is 0.2 .

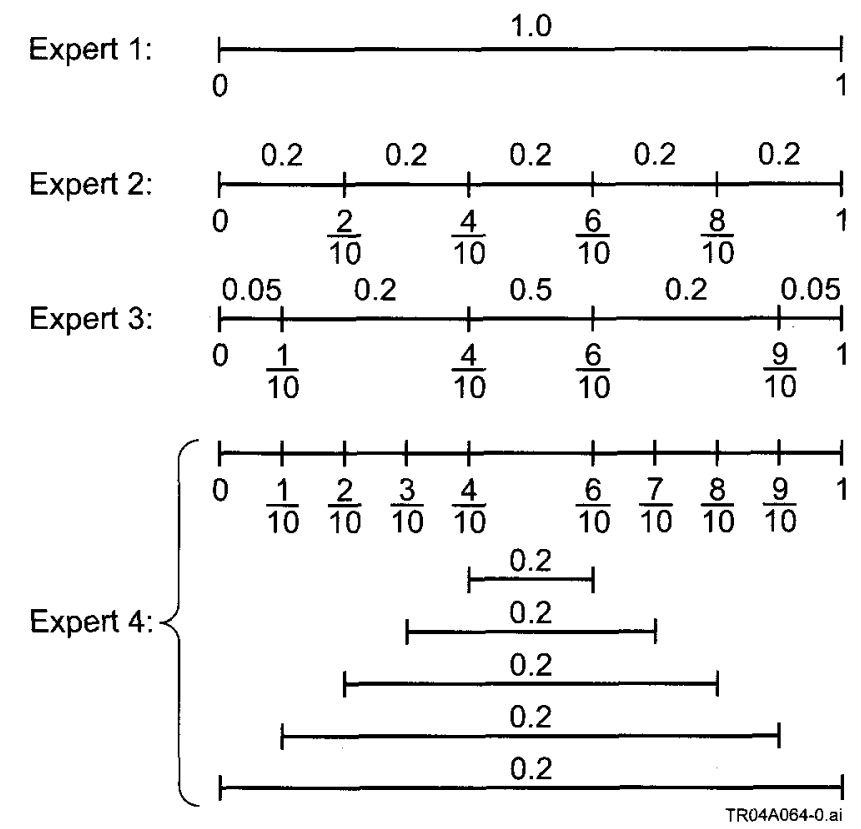

Fig. 6.1. Graphical illustration of uncertainty information in Table 6.2 with variable range $[a, b]$ normalized to $[0,1]$ for notational convenience.

$$
d_{i}(v)= \begin{cases}\operatorname{prob}_{i}\left(I_{i j}\right) / L\left(I_{i j}\right) & \text { if } v \in I_{i j} \\ 0 & \text { otherwise }\end{cases}
$$

for $i=2,3$, where $\operatorname{prob}_{i}\left(I_{i j}\right)$ denotes the probability for interval $I_{i j}$ specified by Expert $i$ and $L\left(I_{i j}\right)$ denotes the length of interval $I_{i j}$. Expert 4 specifies what is, in essence, a BPA assignment for the variable. This specification 
can be converted to a probability distribution in a manner consistent in spirit with the handling of the information supplied by Experts 1, 2 and 3 by assuming a uniform distribution over each of the specified intervals and then weighting each of these distributions by the BPA assigned to the corresponding interval. The outcome of this process for the information supplied by Expert 4 is the density function

$$
d_{4}(v)=\sum_{j=1}^{4} \delta_{4 j}(v) \operatorname{prob}_{4}\left(I_{4 j}\right) / L\left(I_{4 j}\right)
$$

where $\delta_{4 j}(v)=1$ if $v \in I_{4 j}$ and 0 otherwise, $\operatorname{prob}_{4}\left(I_{4 j}\right)$ denotes the amount of probability that can be assigned to $I_{4 j}$ but not to any particular subset of $I_{4 j}$, and $L\left(I_{4 j}\right)$ is the same as in Eq. (6.25).

The distributions obtained from the individual experts can now be combined to obtain a single distribution that characterizes the uncertainty in the variable under consideration. Such "aggregation" of information from multiple sources is a much studied topic. ${ }^{70-74}$ Here, the widely used approach of assigning equal weight (i.e., credibility) to each expert to produce a single distribution is used. Specifically, the resultant density function from this approach is given by

$$
d(v)=\sum_{i=1}^{n E} d_{i}(v) / n E,
$$

where $n E=4$ is the number of experts. The described approach results in each variable in Table 6.1 having an uncertainty distribution of the form shown in Fig. 6.2, with only the values of $a$ and $b$ and associated scaling between $a$ and $b$ changing from variable to variable.

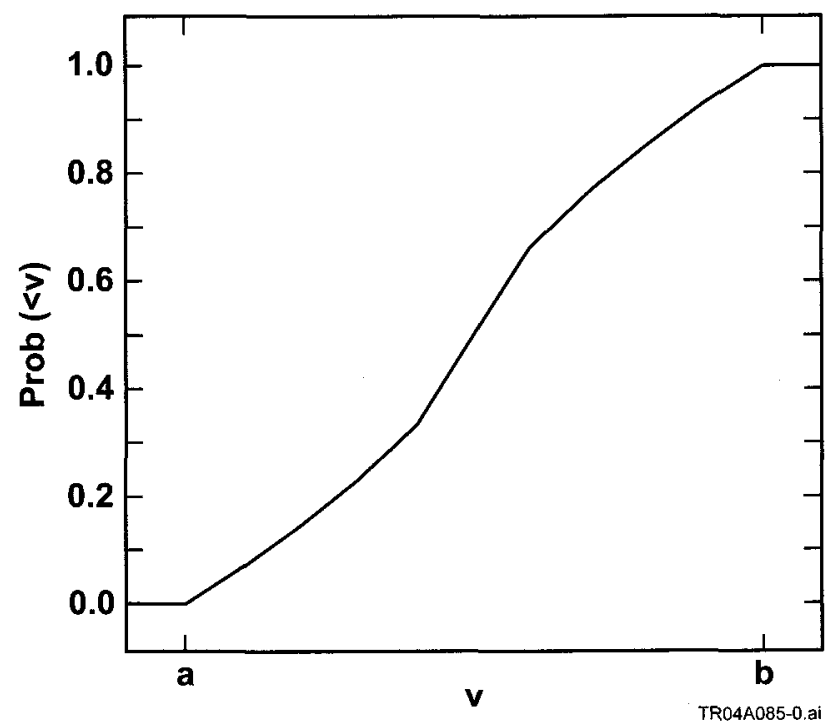

Fig. 6.2. Distribution of a variable over the interval $[a, b]$ derived from the information in Table 6.2 . 
The construction of BPAs from the information in Table 6.2 is now considered. The experts specify intervals and associated probabilities. These probabilities can be interpreted as BPAs for the corresponding intervals (i.e., $I_{11}$ for Expert 1 , and $I_{i j}, j=1,2, \ldots, 5$ for Expert $\left.i, i=2,3,4\right)$. Specifically, the BPA $m_{i}$ associated with Expert $i$ is given by

$$
m_{i}(\mathscr{U})= \begin{cases}\operatorname{prob}_{i}(\mathscr{U}) & \text { if } U \in \mathbb{M}_{i} \\ 0 & \text { otherwise }\end{cases}
$$

for an arbitrary set $\mathscr{U}$ of points from $[a, b]$, where $\mathbb{M}_{1}=\left\{I_{11}\right\}$ and $\mathbb{M}_{i}=\left\{I_{i j}, j=1,2, \ldots, 5\right\}$ for $i=2,3,4$. Analogously to the weighting process implemented in Eq. (6.27) for density functions, the BPAs from the individual experts can be equally weighted to produce a final BPA $m$. In particular, this final BPA is given by

$$
m(U)=\sum_{i=1}^{n E} m_{i}(\Psi) / n E,
$$

where $n E=4$ is the number of experts and $\mathcal{U}$ is an arbitrary subset of points from $[a, b]$. The preceding procedure produces 13 sets with nonzero BPAs (Table 6.3). The indicated approach results in each variable in Table 6.1 having a BPA structure of the form indicated in Fig. 6.3.

Table 6.3. Basic Probability Assignments (BPAs) for a Variable on the Interval $[a, b]$ Derived from the Information in Table 6.2

$$
\begin{aligned}
& m(\Psi)=3 / 10 \quad \text { if } \Psi=I_{1}=[a, b] \\
& =1 / 20 \text { if } U=I_{2}=[a, a+(b-a) / 5) \\
& =1 / 20 \text { if } U=I_{3}=[a+(b-a) / 5, a+2(b-a) / 5) \\
& =9 / 40 \text { if } U=I_{4}=[a+2(b-a) / 5, a+3(b-a) / 5) \\
& =1 / 20 \text { if } U=I_{5}=[a+3(b-a) / 5, a+4(b-a) / 5) \\
& =1 / 20 \text { if } \mathcal{U}=I_{6}=[a+4(b-a) / 5, b] \\
& =1 / 80 \text { if } U=I_{7}=[a, a+(b-a) / 10) \\
& =1 / 20 \text { if } \Psi=I_{8}=[a+(b-a) / 10, a+4(b-a) / 10) \\
& =1 / 20 \text { if } U=I_{9}=[a+6(b-a) / 10, a+9(b-a) / 10) \\
& =1 / 80 \text { if } U=I_{10}=[a+9(b-a) / 10, b] \\
& =1 / 20 \text { if } \mathcal{U}=I_{11}=[a+3(b-a) / 10, a+7(b-a) / 10) \\
& =1 / 20 \text { if } \Psi=I_{12}=[a+2(b-a) / 10, a+8(b-a) / 10) \\
& =1 / 20 \text { if } U=I_{13}=[a+(b-a) / 10, a+9(b-a) / 10) \\
& =\underline{0} \text { otherwise } \\
& 1.0
\end{aligned}
$$



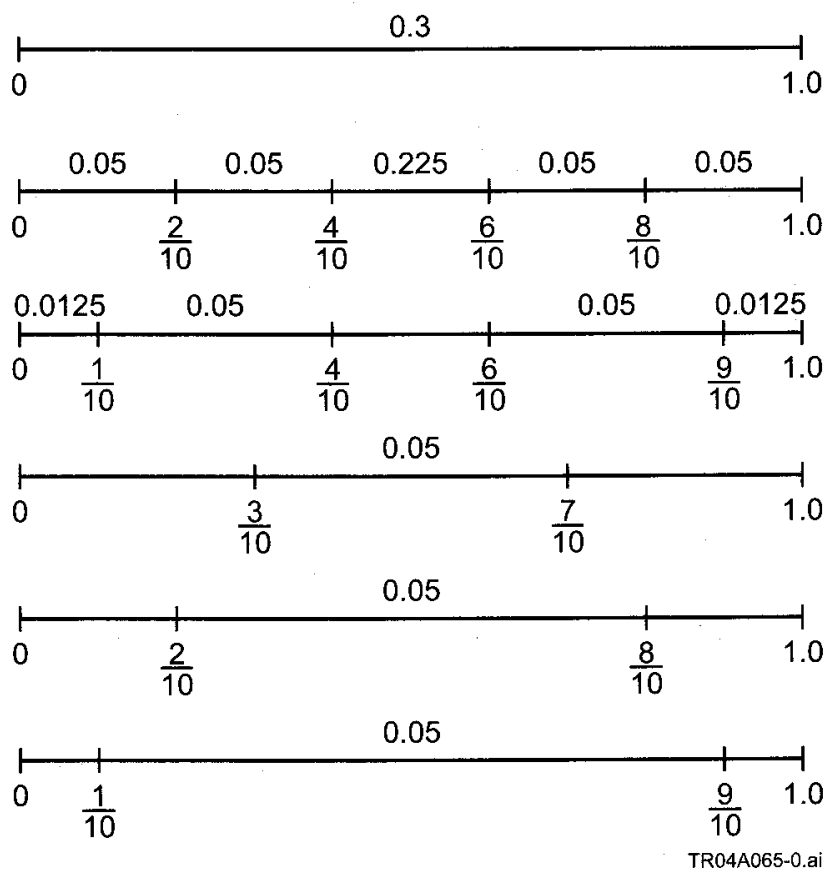

Fig. 6.3. Graphical illustration of the 13 sets in Table 6.3 assigned nonzero BPAs with variable range $[a, b]$ normalized to $[0,1]$ for notational convenience.

\subsection{Example Uncertainty and Sensitivity Analysis Results}

The probabilistically-based uncertainty analysis procedures described in Sects. 6.1 and 6.3 are applied first. Specifically, a random sample of size 200 is generated from the 16 uncertain variables listed in Table 6.1 in consistency with the distributions indicated in Eq. (6.27). The sample elements are vectors of the form

$$
\mathbf{x}_{i}=\left[x_{i 1}, x_{i 2}, \ldots, x_{i 16}\right]
$$

for $i=1,2, \ldots, 200$, where each component $x_{i j}, j=1,2, \ldots, 16$, of $\mathbf{x}_{i}$ corresponds to one of the 16 variables in Table 6.1. Each sample element results in four time-temperature curves (i.e., one curve for each of the two WLs and two SLs) as defined in Eqs. (3.30) - (3.31) and a corresponding failure probability $p F$ as defined in Eq. (4.9) with $n W L$ $=n S L=2$ (Fig. 6.4). The 200 failure probabilities that result from the 200 sample elements indicated in Eq. (6.30) can be displayed as a CCDF (Fig. 6.5), which provides a representation of the epistemic uncertainty associated with the probability that both SLs fail before either WL fails.

A natural adjunct to the uncertainty analysis results presented in Fig. 6.5 is a sensitivity analysis to determine the importance of the individual variables in Table 6.1 in determining the uncertainty in $p F$. In particular, the sampling-based uncertainty analysis in use has generated a mapping

$$
\left[\mathbf{x}_{i}, p F_{i}\right], i=1,2, \ldots, 200
$$




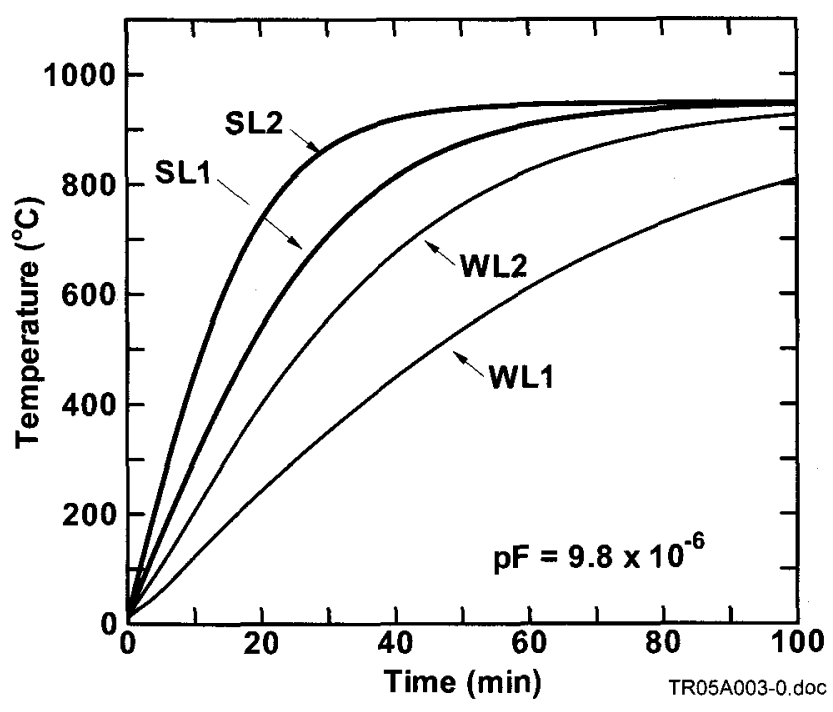

Fig. 6.4. Example WL/SL temperature curves and associated failure probability for sample element $x_{144}$.

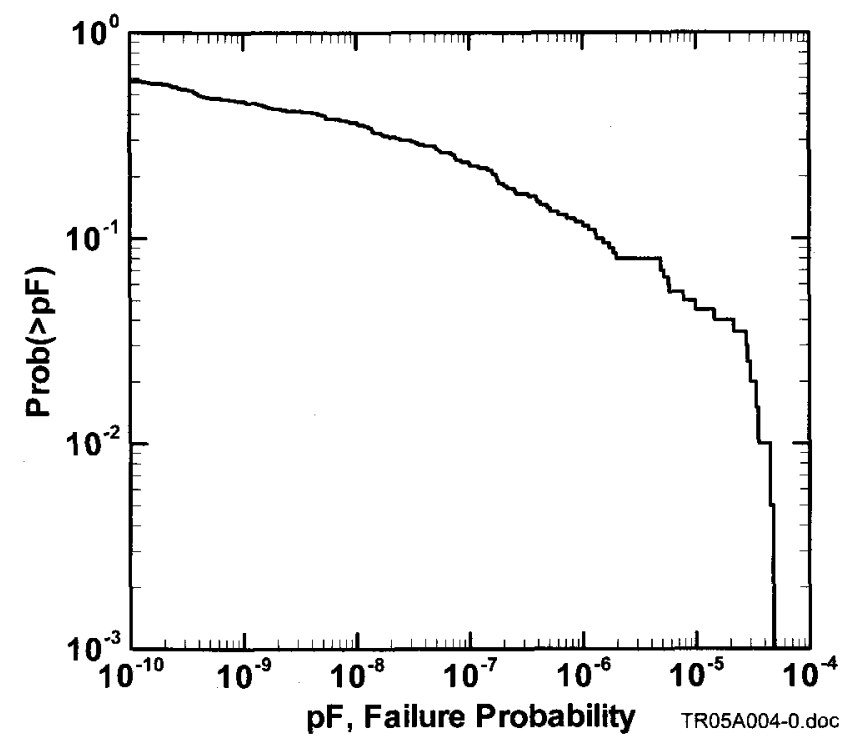

Fig. 6.5. Use of CCDF generated with a random sample of size 200 to display the epistemic uncertainty in the probability $p F$ that both SLs fail before either WL fails.

from the uncertain analysis inputs contained in $\mathbf{x}_{i}$ to the corresponding failure probability $p F_{i}$. A variety of sensitivity analysis procedures exist that can be used in the exploration of this mapping. ${ }^{38-43,75}$ As an example, stepwise regression procedures with log-transformed and rank-transformed data ${ }^{76,77}$ are used to explore this mapping (Table 6.4). In the analysis with $\log$-transformed data, the dependent variable is $\log (p F)$ rather than $p F$, and the independent variables retain their original (i.e., raw or untransformed) values. 
Table 6.4. Stepwise Regression Analysis with Log-Transformed and Rank-Transformed Data for Probability $p F$ that Both SLs Fail before Either WL Fails

\begin{tabular}{ccccccc}
\hline \multirow{2}{*}{ Step $^{\mathrm{a}}$} & \multicolumn{3}{c}{ Log-Transformed Data } & \multicolumn{3}{c}{ Rank-Transformed Data $^{n^{2}}$} \\
\cline { 2 - 7 } Variable $^{\mathrm{b}}$ & SRC $^{\mathrm{c}}$ & $\boldsymbol{R}^{2 \mathrm{~d}}$ & Variable $^{\mathrm{b}}$ & SRRC $^{\mathrm{e}}$ & $\boldsymbol{R}^{2 \mathrm{~d}}$ \\
\hline 1 & $c_{11}$ & 0.77 & 0.59 & $c_{11}$ & 0.84 & 0.67 \\
2 & $c_{71}$ & 0.31 & 0.69 & $c_{71}$ & 0.34 & 0.79 \\
3 & $c_{8}$ & 0.24 & 0.75 & $c_{8}$ & 0.26 & 0.86 \\
4 & $c_{72}$ & 0.18 & 0.78 & $c_{10}$ & -0.16 & 0.89 \\
5 & $c_{9}$ & 0.18 & 0.81 & $c_{9}$ & 0.12 & 0.90 \\
6 & $c_{10}$ & -0.15 & 0.84 & $c_{72}$ & 0.12 & 0.91 \\
7 & $c_{62}$ & 0.11 & 0.85 & $c_{2}$ & 0.10 & 0.92 \\
8 & $c_{42}$ & -0.08 & 0.85 & $c_{42}$ & -0.09 & 0.93 \\
9 & $c_{2}$ & 0.07 & 0.86 & $c_{1}$ & -0.06 & 0.93
\end{tabular}

a Steps in stepwise regression analysis with significance levels of $\alpha=0.02$ and $\alpha=0.05$ required of a variable for entry into and retention in a regression model, respectively.

$\mathrm{b}$ Variables listed in order of selection in regression analysis.

c Standardized regression coefficients (SRCs) in final regression model with log-transformed values for $p F$.

${ }^{\mathrm{d}}$ Cumulative $R^{2}$ value with entry of each variable into regression model.

e Standardized rank regression coefficients (SRRCs) in final regression model with rank-transformed values for all variables.

The regression results with log-transformed and rank-transformed data are similar. In particular, both regression approaches identify $c_{11}$ (standard deviation of normal distribution for SL failure temperature), $c_{71}$ (factor used to account for more rapid heating in SL1 than in the associated WL), and $c_{8}$ (expected value of normal distribution for WL failure temperature) as the most important variables with respect to the observed uncertainty in $p F$. The positive regression coefficients are consistent with patterns observed in the associated scatterplots (Fig. 6.6) and the known effects of these variables. In particular, (i) increasing $c_{11}$ increases the number of low SL failure temperatures and thus increases $p F$, (ii) increasing $c_{71}$ increases the temperature of SL 1 relative to WL 1 and thus increases $p F$, and (iii) increasing $c_{8}$ increases the number of high WL failure temperatures and thus increases $p F$. As is often the case due to the linearizing effects of the rank transformation, the analyses with rank-transformed data result in somewhat higher $R^{2}$ values than is the case for the analyses with raw data.

For perspective, the analysis was also performed for three samples of size 200 , three samples of size 1000 and three samples of size 10,000 (Fig. 6.7). Except for getting better resolution with respect to the likelihood of obtaining very large values for $p F$ (i.e., close to $10^{-3}$ ), the results for the different sample sizes are quite similar. Thus, as is usually the case, the sample size needed to determine where most of the uncertainty is located is not particularly large. Of course, this changes if the goal of the analysis is to identify high consequence but unlikely analysis outcomes. For example, if the goal of the analysis was to determine an epistemic (i.e., degree of belief or subjective) probability that the value for $p F$ exceeds $10^{-6}$, then the same conclusion would be drawn from any of the nine 
CCDFs presented in Fig. 6.7. Specifically, and conditional on the assumptions of the hypothetical example under consideration, this probability is approximately 0.1 .
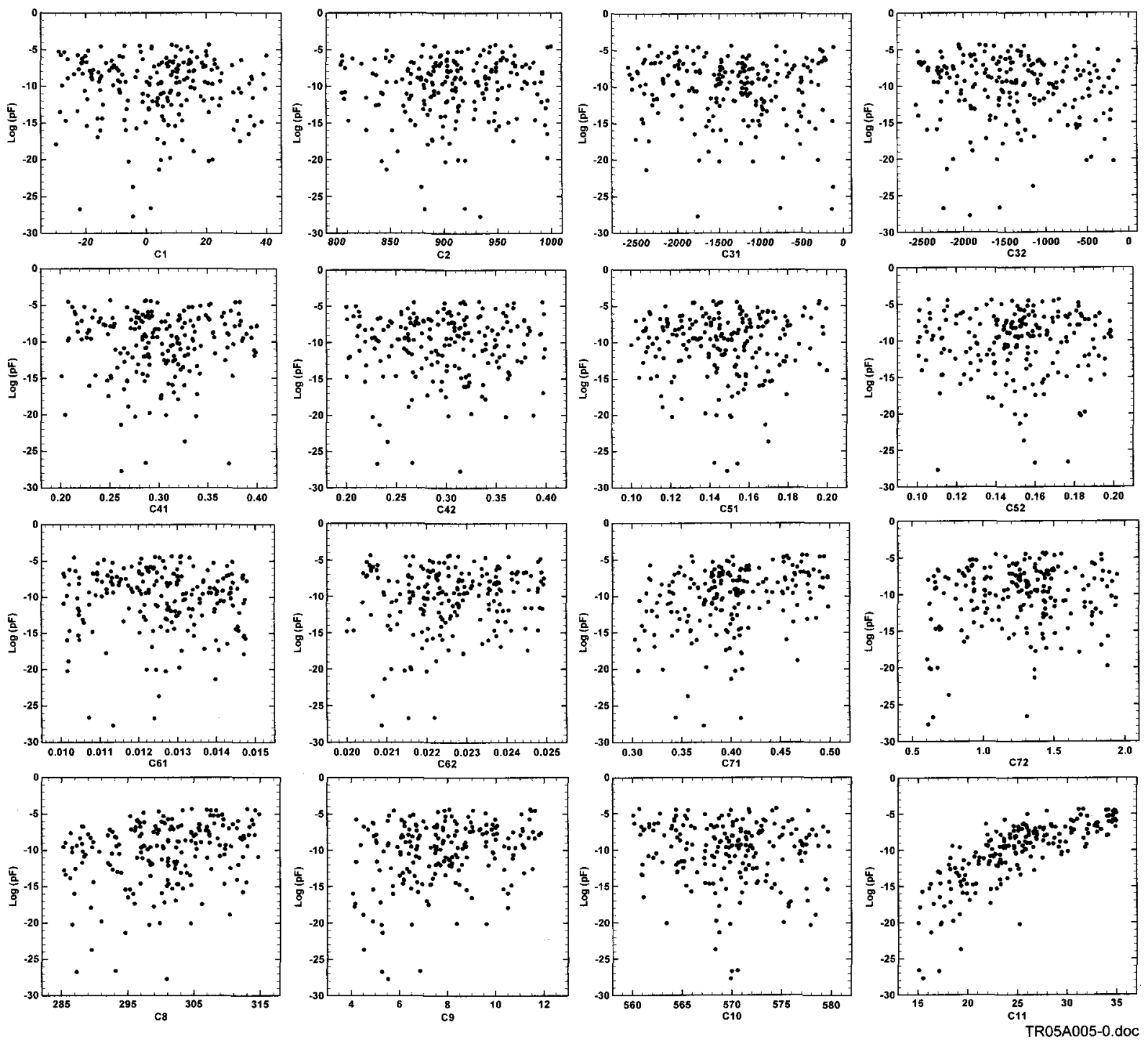

Fig. 6.6. Scatterplots for 16 variables in Table 6.1 sampled in uncertainty analysis of the probability $p F$ that both SLs fail before either WL.

The representation of the epistemic uncertainty associated with $p F$ in the context of evidence theory is now considered. This representation is based on the BPAs for the 16 elements of $\mathbf{x}$ defined in Eq. (6.29) and illustrated in Table 6.3 and Fig. 6.3. In concept, the CCPF and CCBF for $p F$ can be constructed from the evidence space ( $X X$, $\left.\mathbb{X}, m_{X}\right)$ defined in Sect. 6.4 with the Monte Carlo procedure defined in conjunction with Eqs. (6.22) and (6.23). Unfortunately, the large number of sets contained in $\mathbb{X}$, (i.e., $13^{16} \doteq 6.7 \times 10^{17}$ ) makes direct use of this procedure with the evidence space $\left(\AA X, \mathbb{X}, m_{X}\right)$ computationally impracticable. Specifically, it is not computationally possible to carry out an analysis with a sufficiently large sample to assure adequate coverage of all sets contained in $\mathbb{X}$. 
Thus, some approach other than a direct application of the procedures in Eqs. (6.22) and (6.23) with (eX, $\mathbb{X}, m_{X}$ ) must be sought to obtain the CCPF and CCBF for $p F$.
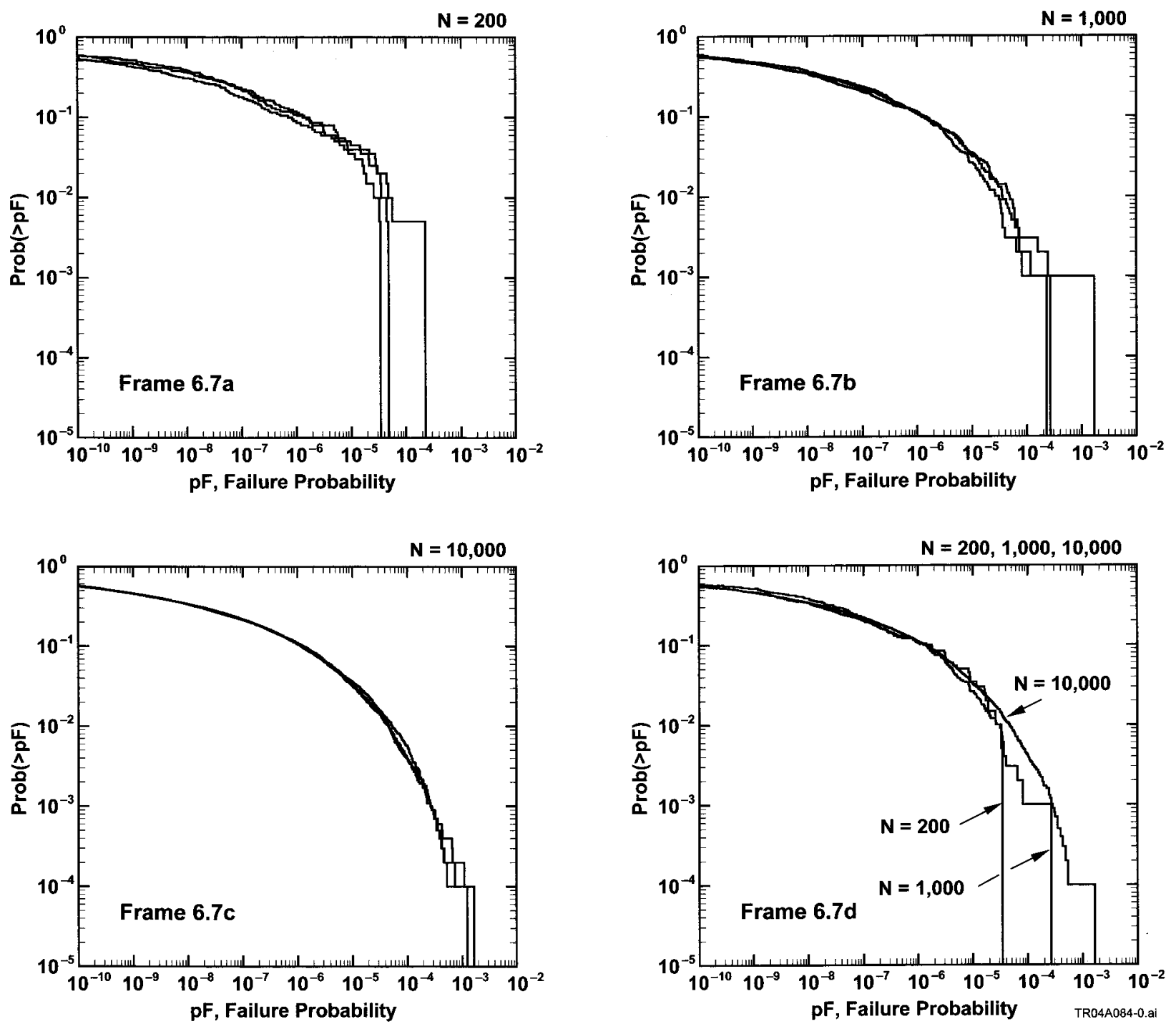

Fig. 6.7. Estimation of epistemic uncertainty in $p F$ with three replicated random samples: (a) $N=$ 200 , (b) $N=1000$, (c) $N=10,000$, and (d) one CCDF from each of the preceding frames.

Fortunately, a computationally practicable approach can be based on the following two ideas. First, if a variable does not affect an analysis outcome of interest, then calculated beliefs and plausibilities are unaffected by the evidence theory structure assigned to this variable. Thus, any convenient structure can be assigned to this variable for use in the construction of CCBFs and CCPFs. Second, the uncertainty bounds represented by CCBFs and CCPFs narrow as the resolution in the evidence spaces assigned to the input variables increases. Intuitively, the resolution in an evidence space increases when its focal elements are subdivided to produce a new evidence space with an increased number of focal elements. Specifically, if the evidence space $\left(\AA_{i}, \mathbb{X}_{i}, m_{i}\right)$ associated with $x_{i}$ is replaced by an evidence space $\left(\stackrel{e}{i}, \tilde{\mathbb{X}}_{i}, \tilde{m}_{i}\right)$ with the properties that (i) all sets in $\tilde{\mathbb{X}}_{i}$ are subsets of sets in $\mathbb{X}_{i}$, (ii) 
any set in $\mathbb{X}_{i}$ can be obtained from a union of sets in $\tilde{\mathbb{X}}_{i}$, and (iii) the BPA for each set $\mathcal{E}$ in $\mathbb{X}_{i}$ is apportioned over the sets in $\tilde{X}_{i}$ contained in $\varepsilon$, then the $\mathrm{CCBF}$ and $\mathrm{CCPF}$ that result from the uncertainty characterization that derives from $\left(X_{i}, \tilde{\mathbb{X}}_{i}, \tilde{m}_{i}\right)$ will be inside the $\mathrm{CCBF}$ and $\mathrm{CCPF}$ that result from the uncertainty characterization that derives from $\left(\mathrm{e} X_{i}, \mathbb{X}_{i}, m_{i}\right)$. Thus, an uncertainty analysis of increasing resolution can be carried out by starting with relatively low resolution evidence spaces and then increasing resolution, and hence computational demands, until acceptably converged CCBFs and CCPFs are obtained. In particular, resolution only needs to be added for the variables that actually affect the analysis outcome of interest. As indicated in conjunction with Table 6.4, sensitivity analysis can be used to identify such variables. The preceding ideas are elaborated on and applied to the example uncertainty analysis problem in the remainder of this section. A formal justification for the mathematical basis of this approach is given in Sect. 6.6.

The approach to the construction of CCBFs and CCPFs when the cardinality of $\triangle$ is large can be represented as an algorithm of the form shown below, where $\mathbf{x}=\left[x_{1}, x_{2}, \ldots, x_{n}\right],\left(\odot X, \mathbb{X}, m_{X}\right)$ is an evidence space constructed from evidence spaces $\left(\mathrm{C}_{i}, \mathbb{X}_{i}, m_{i}\right)$ for the individual elements $x_{i}$ of $\mathbf{x}$, and $f$ is a function that produces the analysis outcome $y=f(\mathbf{x})$ under study:

Step 0. Perform a sensitivity analysis to determine the most important variables $\tilde{x}_{1}, \tilde{x}_{2}, \ldots, \tilde{x}_{n}$ with respect to the uncertainty in $y$, where $\tilde{x}_{1}$ is the most important variable, $\tilde{x}_{2}$ is the next most important variable, and so on.

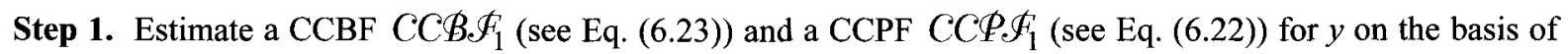
the evidence space $\left(\odot X, \mathbb{S}_{1}, m_{S 1}\right)$ obtained from the original evidence space for $\tilde{x}_{1}$ and degenerate evidence spaces for $\tilde{x}_{2}, \tilde{x}_{3}, \ldots, \tilde{x}_{n}$ in which the sample spaces are assigned BPAs of 1 .

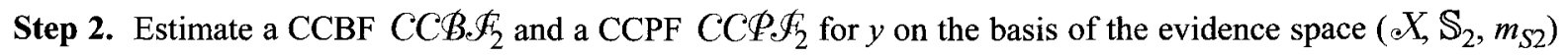
obtained from the original evidence spaces for $\tilde{x}_{1}$ and $\tilde{x}_{2}$ and degenerate evidence spaces for $\tilde{x}_{3}, \tilde{x}_{4}, \ldots, \tilde{x}_{n}$ in which the sample spaces are assigned BPAs of 1 .

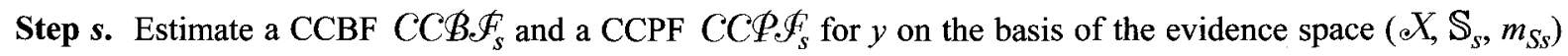
obtained from the original evidence spaces for $\tilde{x}_{1}, \tilde{x}_{2}, \ldots, \tilde{x}_{s}$ and degenerate evidence spaces for $x_{s+1}, x_{s+2}, \ldots, x_{n}$ in which the sample spaces are assigned BPAs of 1.

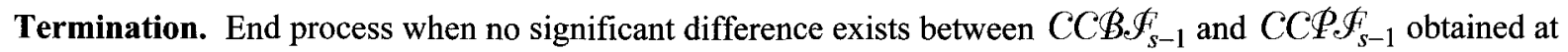

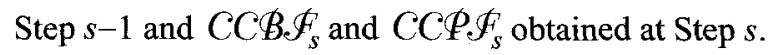

The preceding approach is used in the construction of CCBFs and CCPFs for the failure probability $p F$. Step 0 corresponds to the sensitivity analysis summarized in Table 6.4, which identified (in the rank regression) $c_{11}, c_{71}, c_{8}$, $c_{10}$ and $c_{9}$ as the dominant variables with respect to the uncertainty in $p F$ (i.e., $c_{11} \sim \tilde{x}_{1}, c_{71} \sim \tilde{x}_{2}, c_{8} \sim \tilde{x}_{3}, c_{10} \sim \tilde{x}_{4}$, 
$\left.c_{9} \sim \tilde{x}_{5}\right)$. Steps $1,2,3,4$ and 5 are then carried out with the successive full inclusion of $c_{11}$ to produce $C C B \mathscr{F}_{1}$ and

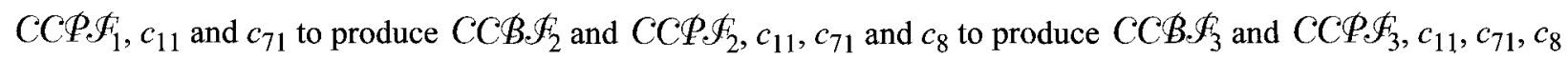

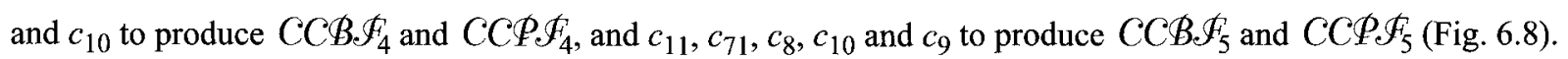

The construction of the indicated CCBFs and CCPFs was carried out with a random sample of size $N=10^{6}$ from the distributions defined by the density functions in Eq. (6.27) and the associated mapping from uncertain analysis inputs to analysis results (i.e., a mapping of the form indicated in Eq. (6.31) but generated with a sample size of $10^{6}$ rather than 200 ).

As illustrated in Fig. 6.8, the CCBFs and CCPFs move closer together as the resolution increases in the evidence spaces for the elements of $\mathbf{x}$. The CCPFs, which are shown in greater detail in Fig. 6.9, are probably close to being converged with the five variables under consideration. The addition of several more variables (i.e., steps in the construction algorithm) may be needed to converge the CCBFs. The CCDF in Figs. 6.8 and 6.9 is the CCDF that derives from sampling distribution used in the estimation of the CCBFs and CCPFs (i.e., the distribution defined by the density functions in Eq. (6.27)); specifically, this CCDF is constructed from the previously indicated sample of size $N=10^{6}$ and associated mapping from uncertain analysis inputs to analysis results. As such, it is one of the many CCDFs for $p F$ that are consistent with the evidence space for $\mathbf{x}$ under consideration (i.e., the evidence space defined by the BPAs for the individual variables defined in Eq. (6.29)).

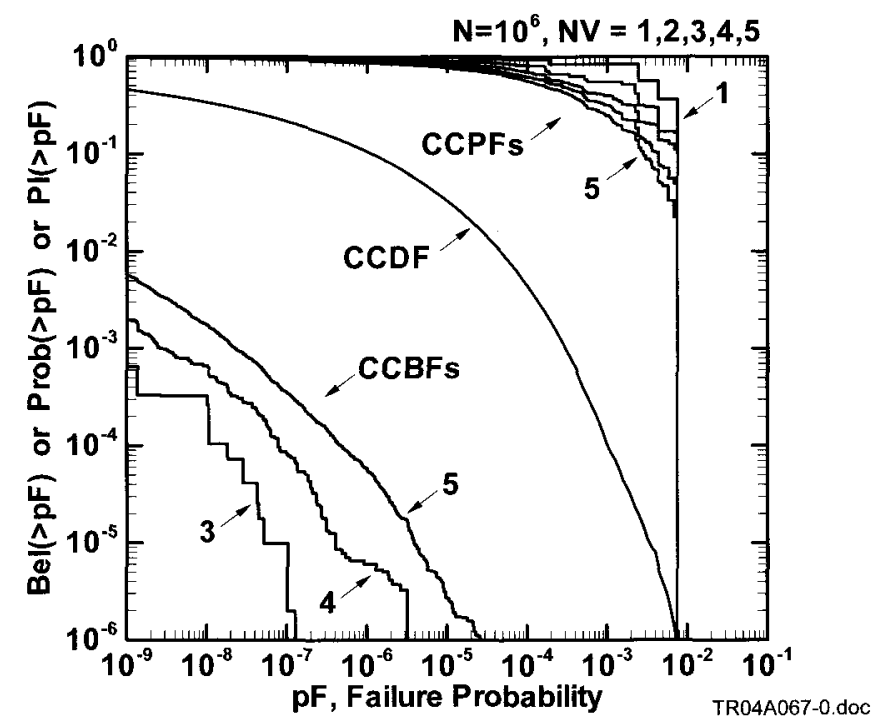

Fig. 6.8. Successive CCBFs (i.e., $C C B \mathscr{F}_{s}, s=1,2$, $\ldots, 5$, with $s=1,2$ off scale) and CCPFs (i.e., $\left.C C P F_{s}, s=1,2, \ldots, 5\right)$ estimated in application of algorithm described in Sect. 6.5 to develop an evidence theory representation of the epistemic uncertainty in the probability $p F$ that both SLs fail before either WL fails. 


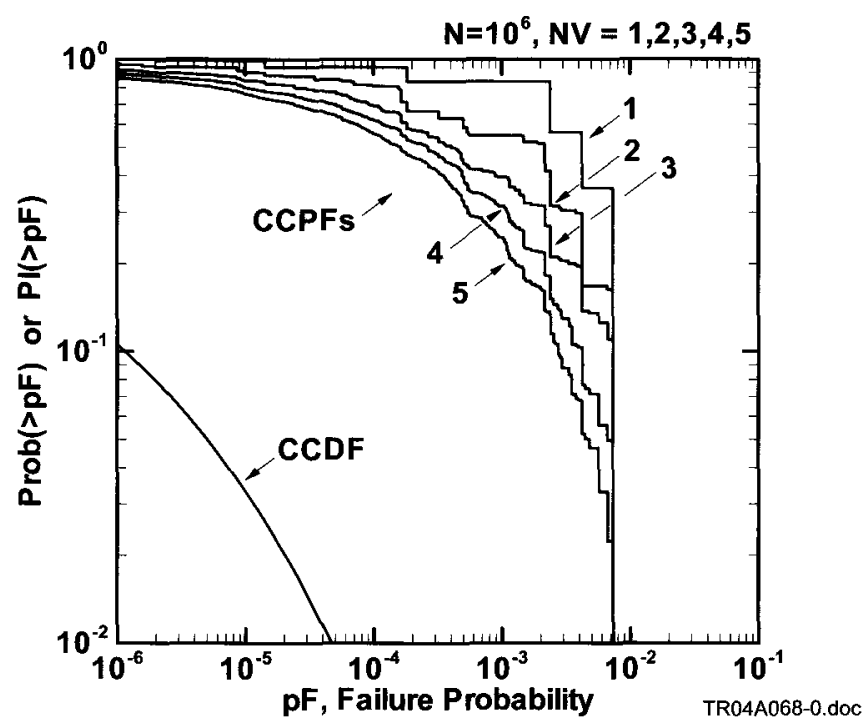

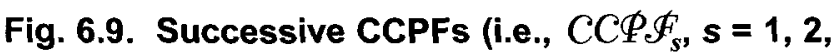
$3,4,5)$ appearing in Fig. 6.8 plotted with shortened intervals on the axes.

The evidence spaces for the individual elements of $\mathbf{X}$ are defined with 13 focal elements. Thus, as the number of steps in the construction algorithm for CCBFs and CCPFs increases, the number of focal elements in the evidence space $\left(\propto X, \mathbb{S}_{s}, m_{S S}\right)$ for $\mathbf{x}$ rapidly increases. For example, consideration of 5,6 and 7 variables results in evidence spaces $\left(\mathcal{e}, \mathbb{S}_{S}, m_{S S}\right), s=5,6,7$, for $\mathbf{x}$ with approximately $3.71 \times 10^{5}, 4.83 \times 10^{6}$ and $6.27 \times 10^{7}$ focal elements, respectively. Adequate sampling of this many focal elements is not possible except for models that are inexpensive to evaluate. A possible numerical solution in this situation is to replace the original evidence spaces for the elements of $\mathbf{x}$ with evidence spaces that have a similar structure but a smaller number of focal elements. For example, such a reduction can be carried out by (i) evaluating the CBF and CPF for a variable, (ii) determining intervals $\left[a_{i}, b_{i}\right]$ of variable values associated with the CBF and CPF values of $i / n$ for $i=0,1, \ldots, n$, and then (iii) assigning a BPA of $1 /(n+1)$ to each interval $\left[a_{i}, b_{i}\right]$ for $i=0,1, \ldots, n$.

\subsection{Justification of Assumptions for Estimation of CCBFs and CCPFs}

Two key assumptions underlie the algorithm presented in Sect. 6.5 for the estimation of the CCBF and CCPF that results from the mapping of an evidence space $\left(\mathrm{e} X, \mathbb{X}, m_{X}\right)$ into an evidence space $\left(Y, \mathbb{Y}, m_{Y}\right)$ by a function $f$ defined on $X$. The first assumption is that, if a variable does not affect an analysis outcome of interest, then the calculated beliefs and plausibilities for this outcome are unaffected by the evidence theory structure assigned to the variable. The second assumption is that the uncertainty bounds represented by CCBFs and CCPFs narrow as the resolution in the evidence spaces assigned to input variables increases. These assumptions are now formalized and 
justified through a sequence of definitions and theorems. In particular, the first assumption is justified by Theorem 6.3 , and the second assumption is justified by Theorem 6.2 .

Intuitively, an evidence space ( $\left.\mathcal{V}, \mathbb{V}, m_{V}\right)$ refines an evidence space ( $\mathscr{U}, \mathbb{U}, m_{U}$ ) provided (i) $\mathcal{U}$ and $\mathcal{V}$ are the same, (ii) the elements of $\mathbb{V}$ are obtained by subdividing (i.e., refining) the elements of $\mathbb{U}$, (iii) the BPA for each element $\mathcal{E}$ of $\mathbb{U}$ is partitioned over the sets into which $\mathcal{E}$ is subdivided, and (iv) the BPA for each element of $\mathbb{V}$ is the sum of the values assigned to this set in the partitioning of the elements of $\mathbb{U}$. The preceding summation is necessary when an element of $\mathbb{V}$ appears in the partitioning of two or more elements of $\mathbb{U}$. The following definition provides a formal statement of the intuitive idea that $\left(\mathcal{V}, \mathbb{V}, m_{V}\right)$ refines $\left(\mathcal{U}, \mathbb{U}, m_{U}\right)$.

Definition 6.1. An evidence space $\left(\mathcal{V}, \mathbb{V}, m_{V}\right)$ is a refinement of an evidence space ( $\left.\mathbb{U}, \mathbb{U}, m_{U}\right)$ provided: (i) $\mathbb{U}=\mathbb{V}$, (ii) the sets $\mathbb{U}$ and $\mathbb{V}$ are related by

$$
\begin{aligned}
\mathbb{U} & =\left\{\mathbb{U}_{i}: i=1,2, \ldots, m\right\} \\
\mathbb{U}_{i} & =\bigcup_{j=1}^{n(i)} V_{i j}, i=1,2, \ldots, m \\
\mathbb{V} & =\left\{D_{i j}: j=1,2, \ldots, n(i), i=1,2, \ldots, m\right\} \\
& =\left\{D_{k}: k=1,2, \ldots, n\right\},
\end{aligned}
$$

and (iii) there exists an $m \times n$ matrix $\mathbf{F}=\left[f_{i k}\right]$ with nonnegative elements such that

$$
\begin{aligned}
& f_{i k}=0 \text { if } \mathcal{V}_{k} \text { is not a subset of } \Psi_{i}, \\
& \sum_{k=1}^{n} f_{i k}=1, i=1,2, \ldots, m,
\end{aligned}
$$

and

$$
m_{V}(\mathcal{E})= \begin{cases}\sum_{i=1}^{m} f_{i k} m_{U}\left(\Psi_{i}\right) & \text { if } \mathcal{E}=V_{k} \in \mathbb{V} \\ 0 & \text { otherwise }\end{cases}
$$

for each subset $\mathcal{E}$ of $\mathscr{U}$.

Theorem 6.1. Suppose the evidence space $\left(\mathcal{V}, \mathbb{V}, m_{V}\right)$ refines the evidence space ( $\left.\mathscr{U}, \mathbb{U}, m_{U}\right)$ and $\mathcal{E}$ is a subset of $U$. Then,

$$
\operatorname{Bel}_{U}(\mathcal{E}) \leq B e l_{V}(\mathcal{E}) \leq P l_{V}(\mathcal{E}) \leq P l_{U}(\mathcal{E})
$$


where the subscripts $U$ and $V$ designate beliefs and plausibilities defined with respect to $\left(\mathcal{U}, \mathbb{U}, m_{U}\right)$ and $(V, \mathbb{V}$, $\left.m_{V}\right)$, respectively.

Proof. The inequality

$$
\operatorname{Bel}_{V}(\mathcal{E}) \leq P l_{V}(\varepsilon)
$$

is a fundamental property of belief and plausibility and follows immediately from their definitions (see Eqs. (6.8) and (6.9)). Thus, the middle inequality in Eq. (6.38) is valid. Further,

$$
\begin{aligned}
\operatorname{Bel}_{U}(\mathcal{E}) & =\sum_{i \in I} m_{U}\left(\mathscr{U}_{i}\right), \text { with } I=\left\{i: \mathscr{U}_{i} \subset \mathcal{E}\right\} \\
& =\sum_{i \in I} \sum_{k=1}^{n} c_{i k} m_{V}\left(\mathcal{V}_{k}\right), \text { with } c_{i k}=f_{i k} m_{U}\left(\mathscr{U}_{i}\right) / \sum_{l=1}^{m} f_{l k} m_{U}\left(\mathscr{U}_{l}\right) \\
& =\sum_{k=1}^{n} m_{V}\left(\mathcal{V}_{k}\right) \sum_{i \in I} c_{i k} \\
& =\sum_{k \in \propto} m_{V}\left(\mathcal{V}_{k}\right) \sum_{i \in I} c_{i k}, \text { with } \mathcal{K}=\left\{k: \mathscr{V}_{k} \subset \mathcal{E}\right\}, \sum_{i \in I} c_{i k}=0 \text { for } k \notin \mathcal{K} \\
& \leq \sum_{k \in\lrcorner \mathcal{K}} m_{V}\left(\mathcal{V}_{k}\right), \text { since } \sum_{i \in I} c_{i k} \leq 1 \\
& =B e l_{V}(\mathcal{E}) .
\end{aligned}
$$

Thus, the left inequality in Eq. (6.38) is established. Finally,

$$
\begin{aligned}
P l_{V}(\mathcal{E}) & =\sum_{k \in \mathcal{K}} m_{V}\left(\mathcal{V}_{k}\right), \text { with } \mathcal{K}=\left\{k: \mathcal{V}_{k} \cap \mathcal{E} \neq \phi\right\} \\
& =\sum_{k \in \mathcal{K}} \sum_{i=1}^{m} f_{i k} m_{U}\left(\mathscr{U}_{i}\right) \\
& =\sum_{i=1}^{m} m_{U}\left(\mathcal{U}_{i}\right) \sum_{k \in \mathcal{O}} f_{i k} \\
& =\sum_{i \in I} m_{U}\left(\mathscr{U}_{i}\right) \sum_{k \in \mathcal{K}} f_{i k}, \text { with } I=\left\{i: \mathscr{U}_{i} \cap \mathcal{E} \neq \phi\right\}, \sum_{k \in \mathcal{K}} f_{i k}=0 \text { for } i \notin I \\
& \leq \sum_{i \in I} m_{U}\left(\mathscr{U}_{i}\right), \text { since } \sum_{k \in \mathcal{O}} f_{i k} \leq 1 \\
& =P l_{U}(\mathcal{E}) .
\end{aligned}
$$

Thus, the right inequality in Eq. (6.38) is valid, which completes the proof.

Theorem 6.2. Suppose the evidence space $\left(\mathcal{V}, \mathbb{V}, m_{V}\right)$ refines the evidence space $\left(\mathcal{U}, \mathbb{U}, m_{U}\right), f$ is a function

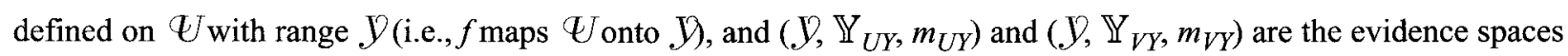
that derive from $\left(\mathscr{U}, \mathbb{U}, m_{U}\right)$ and $\left(\mathcal{V}, \mathbb{V}, m_{V}\right)$ and the mapping from $\mathscr{U}$ to $\mathbb{Y}$ defined by $f$, and $\mathcal{E}$ is a subset of $\mathcal{Y}$. Then, 


$$
B e l_{U Y}(\mathcal{E}) \leq B e l_{V Y}(\mathcal{E}) \leq P l_{V Y}(\mathcal{E}) \leq P l_{U Y}(\mathcal{E})
$$

where the subscripts $U Y$ and $V Y$ designate beliefs and plausibilities defined with respect to $\left(\mathcal{Y}, \mathbb{Y}_{U Y}, m_{U Y}\right)$ and $(I$, $\left.\mathbb{Y}_{V Y}, m_{V Y}\right)$, respectively.

Proof. This result follows immediately from Theorem 6.1 in the following manner:

$$
\begin{aligned}
\operatorname{Bel}_{U Y}(\varepsilon) & =B e l_{U}\left[f^{-1}(\varepsilon)\right] \\
& \leq B e l_{V}\left[f^{-1}(\varepsilon)\right] \\
& \leq P l_{V}\left[f^{-1}(\varepsilon)\right] \\
& \leq P l_{U}\left[f^{-1}(\varepsilon)\right] \\
& =P l_{U Y}(\varepsilon),
\end{aligned}
$$

where the three inequalities follow from Theorem 6.9.

Definition 6.2. Suppose $x_{i}$ are variables with evidence spaces $\left(\mathrm{e}_{i}, \mathbb{X}_{i}, m_{i}\right)$ for $i=1,2, \ldots, n,\left(\mathrm{e} X, \mathbb{X}, m_{X}\right)$ is the corresponding evidence space for $\mathbf{x}=\left[x_{1}, x_{2}, \ldots, x_{n}\right], I$ is a set satisfying $\phi \neq I \subset\{1,2, \ldots, n\}$, and $\left(\mathcal{C}_{i}, \tilde{\mathbb{X}}_{i}, \tilde{m}_{i}\right)$ is

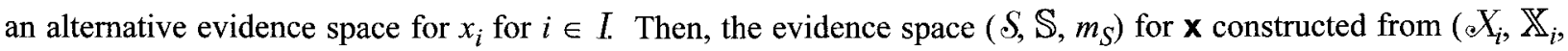
$m_{i}$ ) for $i \in I^{\mathrm{c}}$ and $\left(\mathrm{e} X_{i}, \tilde{\mathbb{X}}_{i}, \tilde{m}_{i}\right)$ for $i \in I$ is called a substitution evidence space for $\mathbf{X}$.

Definition 6.3. Suppose $x_{i} \in \mathrm{e}_{i}$ for $i=1,2, \ldots, n, \mathbf{x}=\left[x_{1}, x_{2}, \ldots, x_{n}\right], f$ is a function defined on $X={ } X_{1} \times{ } X_{2}$ $\times \ldots \times X_{n}$, and $I$ is a set satisfying $\phi \neq I \subset\{1,2, \ldots, n\}$. Then, the following two statements are equivalent: (i) the variables $x_{i}, i \in I$, are nonaffecting with respect to $f$, and (ii) if $\mathbf{x} \in \mathcal{d}, \tilde{\mathbf{x}} \in \mathcal{Q} X$, and $x_{i}=\tilde{x}_{i}$ for $i \in I^{\mathrm{c}}$, then $f(\mathbf{x})=$ $f(\tilde{\mathbf{x}})$.

Theorem 6.3. Suppose $x_{i}$ are variables with evidence spaces $\left({ }^{\circ} X_{i}, \mathbb{X}_{i}, m_{i}\right)$ for $i=1,2, \ldots, n,\left(\odot X, \mathbb{X}, m_{X}\right)$ is the corresponding evidence space for $\mathbf{x}=\left[x_{1}, x_{2}, \ldots, x_{n}\right], I$ is a set satisfying $\phi \neq I \subset\{1,2, \ldots, n\},\left(\propto X_{i}, \tilde{\mathbb{X}}_{i}, \tilde{m}_{i}\right)$ is an alternative evidence space for $x_{i}$ for $i \in I,\left(\odot, \mathbb{S}, m_{S}\right)$ is the substitution evidence space for $\mathbf{x}$ constructed from $\left(\odot X_{i}\right.$,

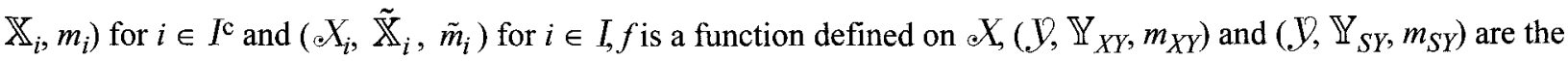
evidence spaces that derive from $\left(\odot X, \mathbb{X}, m_{X}\right)$ and $\left(\odot X, \mathbb{S}, m_{S}\right)$ and the mapping from $\mathrm{e}$ onto $Y$ defined by $f$, the $x_{i}$ for $i \in I$ are nonaffecting with respect to $f$, and $\mathcal{E}$ is a subset of $Y$. Then,

$$
\operatorname{Bel}_{X Y}(\varepsilon)=B e l_{S Y}(\varepsilon) \leq P l_{S Y}(\varepsilon)=P l_{X Y}(\mathcal{E})
$$

where the subscripts $X Y$ and $S Y$ designate beliefs and plausibilities defined with respect to $\left(Y, \mathbb{Y}_{X Y}, m_{X Y}\right)$ and $(\varnothing$, $\left.\mathbb{Y}_{S Y}, m_{S Y}\right)$, respectively.

Proof. For notational convenience, assume that the elements of $\mathbf{X}$ are reordered so that 


$$
I^{c}=\{1,2, \ldots, m\} \text { and } I=\{m+1, m+1, \ldots, n\} .
$$

Thus, the first $m$ elements of $\mathbf{x}$ are assumed to potentially affect the value of $y=f(\mathbf{x})$ and the remaining elements are assumed to be nonaffecting. Further, the following notation is introduced for use later in the proof:

$$
\begin{aligned}
& n(i)=\text { cardinality of } \mathbb{X}_{i} \text { for } i=1,2, \ldots, n, \\
& X(i, j)=\text { element } j \text { of } \mathbb{X}_{i} \text { for } i=1,2, \ldots, n, \\
& \tilde{n}(i)=\text { cardinality of } \tilde{\mathrm{X}}_{i} \text { for } i=m+1, m+2, \ldots, n, \\
& \tilde{X}(i, j)=\text { element } j \text { of } \tilde{\mathbb{X}}_{i} \text { for } i=m+1, m+2, \ldots, n, \\
& I \not \mathcal{A}=\left\{\mathbf{a}: \mathbf{a}=\left[j_{1}, j_{2}, \ldots, j_{m}\right], 1 \leq j_{i} \leq n(i) \text { for } i=1,2, \ldots, n\right\}, \\
& I \mathscr{B}=\left\{\mathbf{b}: \mathbf{b}=\left[j_{m+1}, j_{m+2}, \ldots, j_{n}\right], 1 \leq j_{i} \leq n(i) \text { for } i=m+1, m+2, \ldots, n\right\}, \\
& I C=\left\{\mathbf{c}: \mathbf{c}=\left[j_{m+1}, j_{m+2}, \ldots, j_{n}\right], 1 \leq j_{i} \leq \tilde{n}(i) \text { for } i=m+1, m+2, \ldots, n\right\},
\end{aligned}
$$

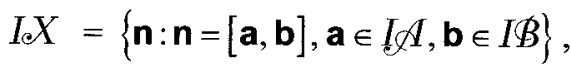

$$
\begin{aligned}
& I S=\{\mathbf{n}: \mathbf{n}=[\mathbf{a}, \mathbf{c}], \mathbf{a} \in I \not A, \mathbf{c} \in I C\},
\end{aligned}
$$

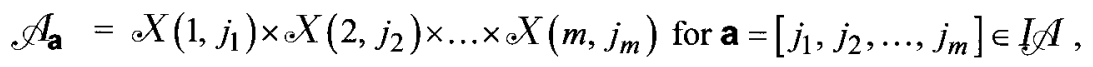

$$
\begin{aligned}
& \mathscr{B}_{\mathbf{b}}={ }_{\mathrm{d}} X\left(m+1, j_{m+1}\right) \times \mathrm{d}\left(m+2, j_{m+2}\right) \times \ldots \times X\left(m, j_{n}\right) \text { for } \mathbf{b}=\left[j_{m+1}, j_{m+2}, \ldots, j_{n}\right] \in I \mathscr{B} \\
& C_{\mathbf{c}}=\tilde{\Theta}\left(m+1, j_{m+1}\right) \times \tilde{X}\left(m+2, j_{m+2}\right) \times \ldots \times \tilde{X}\left(m, j_{n}\right) \text { for } \mathbf{c}=\left[j_{m+1}, j_{m+2}, \ldots, j_{n}\right] \in I C, \\
& m_{A}\left(\mathscr{A}_{\mathbf{a}}\right)=\prod_{k=1}^{m} m_{k}\left[\mathrm{e} X\left(k, j_{k}\right)\right] \text { for } \mathbf{a}=\left[j_{1}, j_{2}, \ldots, j_{m}\right] \in I \mathbb{I} \mathbb{A} \\
& m_{B}\left(\mathscr{B}_{\mathbf{b}}\right)=\prod_{k=m+1}^{n} m_{k}\left[\Theta X\left(k, j_{k}\right)\right] \text { for } \mathbf{b}=\left[j_{m+1}, j_{m+2}, \ldots, j_{n}\right] \in I \mathscr{B} \\
& m_{C}\left(C_{\mathbf{c}}\right)=\prod_{k=m+1}^{n} \tilde{m}_{k}\left[\tilde{X}\left(k, j_{k}\right)\right] \text { for } \mathbf{c}=\left[j_{m+1}, j_{m+2}, \ldots, j_{n}\right] \in I C
\end{aligned}
$$

The preceding notation facilitates a distinction between the elements of $\mathbf{x}$ that affect and do not affect the value of $f(\mathbf{x})$. 
The equality $\operatorname{Bel}_{X Y}(\mathcal{E})=\operatorname{Bel}_{S Y}(\mathcal{E})$ is considered first. The following sets are defined involving $f^{-1}(\mathcal{E})$ :

$$
\begin{aligned}
& I \mathcal{E}_{\odot} X=\left\{\mathbf{n}: \mathbf{n}=[\mathbf{a}, \mathbf{b}] \in I_{e} X, \mathscr{U}=\mathscr{A}_{\mathbf{a}} \times \mathscr{B}_{\mathbf{b}} \subset f^{-1}(\mathcal{E})\right\} \\
& I \not \mathbb{A}_{\mathrm{e}} X=\left\{\mathbf{a}: \mathbf{n}=[\mathbf{a}, \mathbf{b}] \in I \mathcal{E}_{e} X\right\}, \\
& I \mathcal{E S}=\left\{\mathbf{n}: \mathbf{n}=[\mathbf{a}, \mathbf{c}] \in I S, D=\mathscr{A}_{\mathbf{a}} \times C_{\mathbf{c}} \subset f^{-1}(\mathcal{E})\right\},
\end{aligned}
$$

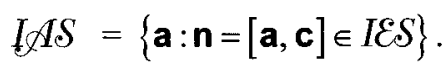

Further, the equality

$$
I \subset I X X=I \not S
$$

follows from the assumption that the variables associated with $I$ (i.e., $x_{m+1}, x_{m+2}, \ldots, x_{n}$ ) are nonaffecting with respect to $f$. Specifically, if $\mathbf{a} \in I \mathscr{A} \cdot X$ and $\mathbf{n}=[\mathbf{a}, \mathbf{b}]$ is an element of $I \varepsilon_{\odot} X$, then $f\left(\mathscr{A}_{\mathbf{a}} \times \mathscr{B}_{\mathbf{b}}\right) \subset \mathcal{E}$; however, because the variables associated with $I$ are nonaffecting, $f\left(\mathscr{A}_{\mathbf{a}} \times C_{\mathbf{c}}\right)=f\left(\mathscr{A}_{\mathbf{a}} \times \mathscr{B}_{\mathbf{b}}\right.$ ) for any $\mathbf{c} \in I C$. Hence, $f\left(\mathscr{A}_{\mathbf{a}} \times C_{\mathbf{c}}\right) \subset \mathcal{E}$,

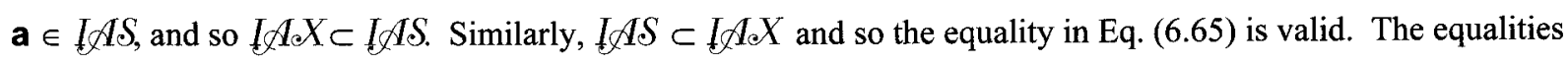

$$
\begin{aligned}
& I \mathcal{E}_{\odot} X=\bigcup_{\mathbf{a} \in I \mathscr{A} X}\{\mathbf{n}: \mathbf{n}=[\mathbf{a}, \mathbf{b}], \mathbf{b} \in I \mathscr{B}\}, \\
& I E S=\bigcup_{\mathbf{a} \in I \not \mathcal{S}}\{\mathbf{n}: \mathbf{n}=[\mathbf{a}, \mathbf{c}], \mathbf{c} \in I C\}
\end{aligned}
$$

also derive from the assumption that the variables associated with $I$ are nonaffecting.

From the underlying assumption of independence among the elements of $\mathbf{x}$,

$$
m_{X}\left(\mathscr{A}_{\mathbf{a}} \times \mathscr{B}_{\mathbf{b}}\right)=m_{A}\left(\mathscr{A}_{\mathbf{a}}\right) m_{B}\left(\mathscr{B}_{\mathbf{b}}\right)
$$

for $[\mathbf{a}, \mathbf{b}] \in I \odot X$, and

$$
m_{S}\left(\mathscr{A}_{\mathbf{a}} \times C_{\mathbf{c}}\right)=m_{A}\left(\mathscr{A}_{\mathbf{a}}\right) m_{C}\left(C_{\mathbf{c}}\right)
$$

for $[\mathbf{a}, \mathbf{c}] \in I S$. Further, the equalities

$$
\sum_{\mathbf{b} \in I B} m_{C}\left(B_{\mathbf{b}}\right)=1
$$

and

$$
\sum_{\mathbf{c} \in I C} m_{C}\left(C_{\mathbf{c}}\right)=1
$$

follow from the assumption that the elements of $\mathbf{x}$ associated with I are nonaffecting with respect to $f$. 
The belief $\operatorname{Bel}_{X Y}(\mathcal{E})$ is given by

$$
\begin{aligned}
& \operatorname{Bel}_{X Y}(\mathcal{E})=\sum_{[\mathbf{a}, \mathbf{b}] \in I \mathcal{E}_{\mathcal{O}} X} m_{X}\left(\mathscr{A}_{\mathbf{a}} \times \mathscr{B}_{\mathbf{b}}\right) \\
& =\sum_{\mathbf{a} \in I \notin \mathbb{I} X X} \sum_{\mathbf{b} \in I \mathscr{B}} m_{X}\left(\mathscr{A}_{\mathbf{a}} \times \mathscr{B}_{\mathbf{b}}\right) \\
& =\sum_{\mathbf{a} \in\lfloor\mathscr{A} X} m_{A}\left(\mathscr{A}_{\mathbf{a}}\right) \sum_{\mathbf{b} \in I \mathscr{B}} m_{B}\left(\mathscr{B}_{\mathbf{b}}\right) \\
& =\sum_{\mathbf{a} \in I_{\mathbb{Q}} X} m_{A}\left(\mathscr{A}_{\mathbf{a}}\right) \text {, }
\end{aligned}
$$

where the second, third and fourth equalities follow from Eqs. (6.66), (6.68) and (6.70), respectively. Similarly,

$$
\begin{aligned}
& \operatorname{Bel}_{S Y}(\mathcal{E})=\sum_{[\mathbf{a}, \mathbf{c}] \in I \mathcal{S} S} m_{S}\left(. \mathscr{A}_{\mathbf{a}} \times C_{\mathbf{c}}\right) \\
& =\sum_{\mathbf{a} \in I \mathbb{I} S} \sum_{\mathbf{c} \in I C} m_{S}\left(\mathscr{A}_{\mathbf{a}} \times C_{\mathbf{c}}\right) \\
& =\sum_{\mathbf{a} \in I \not \perp S} m_{A}\left(\mathscr{A}_{\mathbf{a}}\right) \sum_{\mathbf{c} \in I C} m_{C}\left(C_{\mathbf{c}}\right) \\
& =\sum_{\mathbf{a} \in[\mathscr{A S}} m_{A}\left(\mathscr{A}_{\mathbf{a}}\right) \text {, }
\end{aligned}
$$

where the second, third and fourth equalities follow from Eqs. (6.67), (6.69) and (6.71), respectively. The equality $B e l_{X Y}(\mathcal{E})=B e l_{S Y}(\mathcal{E})$ now follows from Eqs. (6.72) and (6.73) and the equality of $I \subset \mathcal{L} X$ and $I \& \mathcal{L}$ indicated in Eq. (6.65).

The equality $P l_{X Y}(\mathcal{E})=P l_{S Y}(\mathcal{E})$ follows by an argument analogous to the one used to establish the equality $\operatorname{Bel}_{X Y}(\mathcal{E})=\operatorname{Bel}_{S Y}(\mathcal{E})$. The only difference is the consideration of sets that intersect $\mathcal{E}$ rather than sets that are contained in $\varepsilon$. Further, the inequality $B l_{S Y}(\mathcal{E}) \leq P l_{S Y}(\mathcal{E})$ is a basic property of belief and plausibility. Thus, theorem is established. 


\section{Comparison with Previous (Bohn) Analysis}

Bohn developed an approach to the treatment of uncertainty in WL/SL temperature curves (App. I) that is different from the approaches presented in Sect. 6. In Bohn's approach, the uncertainty in the temperature curve for a given link and the variability in the failure temperature for that link are combined into a single distribution. The distributions that result from this combination procedure are then used in the calculation of $\overline{p F}$ (i.e., the expected value for probability of loss of assured safety, with the indicated expectation being calculated over epistemic uncertainty in the time-dependent temperature curves for the individual links). Bohn also presents results for three test problems. Although Bohn's procedure for determining $\overline{p F}$ in the presence of uncertainty is less general than the sampling-based procedure for uncertainty analysis presented in Sect. 6, his solutions to the test problems can be used for an independent verification of the procedures developed in this presentation.

In the following, a formal development of Bohn's approach is presented (Sect. 7.1), and the results obtained with Bohn's approach and the procedures introduced in earlier sections of this presentation are shown to be the same (Sect. 7.2).

\subsection{Bohn's Presentation for $\overline{p F}$ with Uncertainty in Temperature Curves}

A derivation for Bohn's representation for $\overline{p F}$ with an incorporation of uncertainty in time-dependent temperature curves follows. This derivation is presented for two reasons. First, the original derivation by Bohn is incomplete and difficult to follow. Second, the original derivation contains an oversight that under certain conditions leads to erroneous values for $\overline{p F}$. Without a careful derivation of the representation used for $\overline{p F}$, this error is difficult to identify and explain. This error is manifested in the third of Bohn's three test problems as will be discussed later.

For simplicity, a system with two SLs and one WL is considered. The basic relationship used in Bohn's development to define probability of loss of assured safety in the presence of time-dependent temperature uncertainty is

$$
\begin{aligned}
\overrightarrow{p F}(t) & =\text { probability that all links fail before time } t \text { with both SLs failing before the WL fails } \\
& =\int_{0}^{t} C D F_{S L 1}(\tau) C D F_{S L 2}(\tau) \mathrm{d}\left[C D F_{W L}(\tau)\right] \\
& =\int_{0}^{t} C D F_{S L 1}(\tau) C D F_{S L 2}(\tau)\left[\mathrm{d} C D F_{W L}(\tau) / \mathrm{d} \tau\right] \mathrm{d} \tau
\end{aligned}
$$

where the first integral is a Riemann-Stieltjes integral, the second integral is the corresponding Riemann integral, and $C D F_{S L 1}(\tau), C D F_{S L 2}(\tau)$ and $C D F_{W L}(\tau)$ are defined by

$C D F_{S L 1}(\tau)=$ probability that SL 1 fails between time 0 and time $\tau$, 
$C D F_{S L 2}(\tau)=$ probability that SL 2 fails between time 0 and time $\tau$,

and

$C D F_{W L}(\tau)=$ probability that WL fails between time 0 and time $\tau$,

respectively.

The integrals defining $p F(t)$ in Eq. (7.1) are obtained from the approximating sums

$$
\begin{aligned}
\overline{p F}(t) & \cong \sum_{i=1}^{n} C D F_{S L 1}\left(\tau_{i-1}\right) C D F_{S L 2}\left(\tau_{i-1}\right)\left[C D F_{W L}\left(\tau_{i}\right)-C D F_{W L}\left(\tau_{i-1}\right)\right] \\
& =\sum_{i=1}^{n} C D F_{S L 1}\left(\tau_{i-1}\right) C D F_{S L 2}\left(\tau_{i-1}\right)\left[\frac{C D F_{W L}\left(\tau_{i}\right)-C D F_{W L}\left(\tau_{i-1}\right)}{\Delta \tau_{i}}\right] \Delta \tau_{i}
\end{aligned}
$$

for $0=\tau_{0}<\tau_{1}<\ldots<\tau_{n}=t, \Delta \tau_{i}=\tau_{i}-\tau_{i-1}$, and

$C D F_{S L 1}\left(\tau_{i-1}\right)=$ probability that SL1 fails between time 0 and time $\tau_{i-1}$,

$C D F_{S L 2}\left(\tau_{i-1}\right)=$ probability that SL2 fails between time 0 and time $\tau_{i-1}$,

$C D F_{W L}\left(\tau_{i}\right)-C D F_{W L}\left(\tau_{i-1}\right)=$ probability that the WL fails between time $\tau_{i-1}$ and time $\tau_{i}$.

The two integrals in Eq. (7.1) result in the limit as $\Delta \tau_{i}$ goes to zero. The preceding derivation for $\overline{p F}(t)$ is predicated on the assumption that the failures of the three links are independent.

The next step in the development is to obtain representations for $C D F_{S L 1}(\tau), C D F_{S L 2}(\tau)$ and $C D F_{W L}(\tau)$. These representations are based on the assumption that there is variability in the temperatures at which the individual links fail and also uncertainty in the temperatures of the individual links at each point in time. In particular, the following density functions are assumed to be known:

$\operatorname{FSL1}(T)=$ density function for SL 1 failure temperature,

$\operatorname{FSL2}(T)=$ density function for SL 2 failure temperature,

$F W L(T)=$ density function for WL failure temperature,

$\operatorname{TSL} 1(T \mid \tau)=$ density function for SL 1 temperature at time $\tau$,

$\operatorname{TSL} 2(T \mid \tau)=$ density function for SL 2 temperature at time $\tau$,

$T W L(T \mid \tau)=$ density function for WL temperature at time $\tau$. 
In addition, the tacit assumptions are made that the possible time-temperature curves for the individual links are nondecreasing and that a link fails instantly if it reaches its failure temperature. The density function $F W L$ corresponds to the density function $f W L_{1}$ introduced in Eq. (3.3), and the density functions $F S L 1$ and $F S L 2$ correspond to the density functions $f S L_{1}$ and $f S L_{2}$ introduced in Eq. (3.4).

Given the preceding density functions, $C D F_{S L 1}(\tau), C D F_{S L 2}(\tau)$ and $C D F_{W L}(\tau)$ are defined by

$$
\begin{aligned}
& C D F_{S L 1}(\tau)=\int_{-\infty}^{\infty}\left[\int_{-\infty}^{T} F S L 1\left(T_{F}\right) \mathrm{d} T_{F}\right] \operatorname{TSL} 1(T \mid \tau) \mathrm{d} T \\
& C D F_{S L 2}(\tau)=\int_{-\infty}^{\infty}\left[\int_{-\infty}^{T} F S L 2\left(T_{F}\right) \mathrm{d} T_{F}\right] T S L 2(T \mid \tau) \mathrm{d} T
\end{aligned}
$$

and

$$
C D F_{W L}(\tau)=\int_{-\infty}^{\infty}\left[\int_{-\infty}^{T} F W L\left(T_{F}\right) \mathrm{d} T_{F}\right] T W L(T \mid \tau) \mathrm{d} T
$$

respectively. In the preceding, $-\infty$ and $\infty$ are used as limits of integration for notational convenience, with the definitions of the density functions effectively resulting in integrals over finite intervals.

When a finite temperature interval $[T M I N, T M A X]$ is under consideration, the representation for $C D F_{S L 1}(\tau)$ is

$$
\begin{aligned}
C D F_{S L 1}(\tau) & =\int_{T M I N}^{T M A X}\left[\int_{T M I N}^{T} F S L 1\left(T_{F}\right) \mathrm{d} T_{F}\right] T S L 1(T \mid \tau) \mathrm{d} T \\
& \cong \sum_{i=1}^{n}\left[\int_{T M N N}^{T_{i-1}} F S L 1\left(T_{F}\right) \mathrm{d} T_{F}\right] T S L 1\left(T_{i-1} \mid \tau\right) \Delta T_{i}
\end{aligned}
$$

where TMIN $=T_{0}<T_{1}<\ldots<T_{n}=T M A X$ in the approximation, $\Delta T_{i}=T_{i}-T_{i-1}$, and the integral defining $C D F_{S L 1}(\tau)$ is obtained in the limit as $\Delta T_{i}$ goes to zero. In the preceding,

$$
\int_{T M I N}^{T_{i-1}} F S L 1\left(T_{F}\right) \mathrm{d} T_{F}
$$

is the probability that SL 1 fails at a temperature less than $T_{i-1}$, and

$$
\operatorname{TSL1}\left(T_{i-1} \mid \tau\right) \Delta T_{i}
$$

is an approximation to the probability that SL 1 experiences a temperature between $T_{i-1}$ and $T_{i}$ at time $\tau$. As a result, the product

$$
\left[\int_{T M I N}^{T_{i-1}} F S L 1\left(T_{F}\right) \mathrm{d} T_{F}\right] T S L 1\left(T_{i-1} \mid \tau\right) \Delta T_{i}
$$


approximates the probability of the event "SL 1 fails at a temperature less than $T_{i-1}$ and also reaches a temperature between $T_{i-1}$ and $T_{i}$ at time $\tau$." Thus, the summation in Eq. (7.12) approximates the probability that SL 1 has failed by time $\tau$, and the corresponding integral is equal to this probability. The preceding derivation for $C D F_{S L 1}(\tau)$ has two underlying assumptions. First, the possible time dependent temperature curves whose behavior is summarized in the density functions $\operatorname{TSL} 1(T \mid \tau)$ are nondecreasing. Second, the failure temperatures characterized by $F S L 1(T)$ and the time dependent temperatures characterized by $T S L 1(T \mid \tau)$ are independent. The functions $C D F_{S L 2}(\tau)$ and $C D F_{W L}(\tau)$ are obtained in an analogous manner.

In concept, $\overline{p F}(t)$ in Eq. (7.1) is now determined by the definitions of $C D F_{S L 1}(\tau), C D F_{S L 2}(\tau)$ and $C D F_{W L}(\tau)$ in Eqs. (7.9) - (7.11) once the density functions in Eqs. (7.3) - (7.8) are specified. In general, the evaluation of $\overline{p F}(t)$ requires a numerical integration because no simple forms for $C D F_{S L 1}(\tau), C D F_{S L 2}(\tau)$ and $C D F_{W L}(\tau)$ will be available for most potential specifications for the density functions in Eqs. (7.3) - (7.8).

The numerical procedures used for the evaluation in $\overline{p F}(t)$ in Bohn's development are based on a simplification of the integrals defining $C D F_{S L 1}(\tau), C D F_{S L 2}(\tau)$ and $C D F_{W L}(\tau)$ that is obtained by restricting the density functions in Eqs. (7.3) - (7.8) to either normal or lognormal distributions. More specifically, the density functions $F S L 1(T)$ and $\operatorname{TSL} 1(T \mid \tau)$ associated with SL 1 are required to be all normal or all lognormal. Similarly, the density functions $F S L 2(T)$ and $T S L 2(T \mid \tau)$ are required to be all normal or all lognormal, and an analogous requirement holds for $F W L(T)$ and $T W L(T \mid \tau)$. As shown below, the indicated restrictions result in a simplification of the integral representations for $C D F_{S L 1}(\tau), C D F_{S L 2}(\tau)$ and $C D F_{W L}(\tau)$.

The simplification of $C D F_{S L 1}(\tau)$ is considered first for the following two cases: (i) $F S L 1(T)$ corresponds to a normal distribution with mean $\mu_{F S L 1}$ and standard deviation $\sigma_{F S L 1}$ (i.e., $F S L 1(T) \sim N\left(\mu_{F S L 1}, \sigma_{F S L 1}\right)$ ) and $T S L 1(T \mid \tau)$ corresponds to a normal distribution with mean $\mu_{T S L 1}(\tau)$ and standard deviation $\sigma_{T S L 1}(\tau)$ (i.e., $T S L 1(T \mid \tau) \sim$ $\left.N\left[\mu_{T S L 1}(\tau), \sigma_{T S L 1}(\tau)\right]\right)$, and (ii) $F S L 1(T)$ corresponds to a lognormal distribution with median $m_{F S L 1}$ and standard deviation $\beta_{F S L 1}$ for $\ln T$ (i.e., $\left.F S L 1(T) \sim L N\left(m_{F S L 1}, \beta_{F S L 1}\right)\right)$ and $T S L 1(T \mid \tau)$ corresponds to a lognormal distribution with median $m_{T S L 1}(\tau)$ and standard deviation $\beta_{T S L 1}(\tau)$ for $\ln T$ (i.e., $\left.T S L 1(T \mid \tau) \sim L N\left[m_{T S L 1}(\tau), \beta_{T S L 1}(\tau)\right]\right)$.

Case (i) is treated first. Determination of $C D F_{S L 1}(\tau)$ involves consideration of two sets of temperatures:

$$
S_{F S L 1}=\left\{T_{F}: T_{F} \text { a possible failure temperature for SL } 1\right\}
$$

and

$$
\mathcal{S}_{T S L 1}(\tau)=\{T: T \text { a possible temperature for SL } 1 \text { at time } \tau\} .
$$

By assumption, the temperatures in $S_{F S L 1}$ and $S_{T S L 1}(\tau)$ have the distributions $N\left(\mu_{F S L 1}, \sigma_{F S L 1}\right)$ and $N\left[\mu_{T S L 1}(\tau)\right.$, $\left.\sigma_{T S L 1}(\tau)\right]$. In turn, 


$$
C D F_{S L 1}(\tau)=\operatorname{prob}\left(S_{F}\right)
$$

where prob denotes probability and

$$
\begin{aligned}
S_{F} & =\left\{\left[T_{F}, T\right]: T_{F} \in S_{F S L 1}, T \in S_{T S L 1}(\tau) \text { and } T_{F} \leq T\right\} \\
& =\left\{\left[T_{F}, T\right]: T_{F} \in \mathcal{S}_{F S L 1}, T \in S_{T S L 1}(\tau) \text { and } T_{F}-T \leq 0\right\}
\end{aligned}
$$

Because $T_{F} \sim N\left(\mu_{F S L 1}, \sigma_{F S L 1}\right)$ and $T \sim N\left[\mu_{T S L 1}(\tau), \sigma_{T S L 1}(\tau)\right]$, it follows that $T_{F}-T \sim N\left\{\mu_{F S L 1}-\mu_{T S L 1}(\tau),\left[\sigma_{F S L 1}^{2}+\right.\right.$ $\left.\left.\sigma_{T S L 1}^{2}(\tau)\right]^{1 / 2}\right\}$. Thus, $\operatorname{prob}\left(S_{F}\right)$ is simply the probability that a normally distributed variable (i.e., $\left.T_{F}-T\right)$ has a value less than or equal to zero, which can be obtained from the standard normal distribution. In particular,

$$
\begin{aligned}
C D F_{S L 1}(\tau) & =\operatorname{prob}\left(S_{F}\right) \\
& =\Phi\left\{\frac{0-\left[\mu_{F S L 1}-\mu_{T S L 1}(\tau)\right]}{\left[\sigma_{F S L 1}^{2}+\sigma_{T S L 1}^{2}(\tau)\right]^{1 / 2}}\right\} \\
& =\Phi\left\{\frac{\mu_{T S L 1}(\tau)-\mu_{F S L 1}}{\left[\sigma_{F S L 1}^{2}+\sigma_{T S L 1}^{2}(\tau)\right]^{1 / 2}}\right\},
\end{aligned}
$$

where

$$
\Phi(x)=\int_{-\infty}^{x}(1 / \sqrt{2 \pi}) \exp \left(-v^{2} / 2\right) d v
$$

is the cumulative distribution function for the standard normal distribution (i.e., $\Phi(x)$ is the probability that $v$ has a value less than or equal to $x$ when $v \sim N(0,1)$; see Eq. (1.21) in Ref. [2]).

Use of the representation in Eq. (7.20) in the evaluation of $C D F_{S L 1}(\tau)$ offers the potential for computational savings in the evaluation of $\overline{p F}(t)$ as defined in Eq. (7.1) (e.g., see Eqs. (2.43), (2.44) and (2.47)). In particular, the expression in Eq. (7.9) involving two iterated integrals is replaced by the expression in Eq. (7.21) that involves only one integral. Further, the evaluation of $\Phi(x)$ has been extensively studied and efficient procedures for its numerical evaluation are available.

Case (ii) is now considered. This case is effectively the same as Case (i) except for the use of $\ln T_{F}$ and $\ln T$ in place of $T_{F}$ and $T$ in the definition of the set $S_{F}$ in Eq. (7.19). In particular, the definition of $S_{F}$ is now

$$
S_{F}=\left\{\left[T_{F}, T\right]: T_{F} \in S_{F S L 1}, T \in S_{T S L 1}(\tau) \text { and } \ln \left(T_{F} / T\right) \leq 0\right\}
$$

Because $\ln T_{F} \sim N\left[\ln \left(m_{F S L 1}\right), \beta_{F S L 1}\right]$ and $\ln T \sim N\left\{\ln \left[m_{T S L 1}(\tau)\right], \beta_{T S L 1}(\tau)\right\}$, it follows that $\ln \left(T_{F} / T\right) \sim N\left\{\ln \left[m_{F S L 1} /\right.\right.$ $\left.\left.m_{T S L 1}(\tau)\right],\left[\beta_{F S L 1}^{2}+\beta_{T S L 1}^{2}(\tau)\right]^{1 / 2}\right\}$. In turn, 


$$
\begin{aligned}
C D F_{S L 1}(\tau) & =\operatorname{prob}\left(S_{F}\right) \\
& =\Phi\left\{\frac{0-\ln \left[m_{F S L 1} / m_{T S L 1}(\tau)\right]}{\left[\beta_{F S L 1}^{2}+\beta_{T S L 1}^{2}(\tau)\right]^{1 / 2}}\right\} \\
& =\Phi\left\{\frac{\ln \left[m_{T S L 1}(\tau) / m_{F S L 1}\right]}{\left[\beta_{F S L 1}^{2}+\beta_{T S L 1}^{2}(\tau)\right]^{1 / 2}}\right\}
\end{aligned}
$$

with $\Phi(x)$ defined in Eq. (7.21).

Analogous representations hold for $C D F_{S L 2}(\tau)$ and $C D F_{W L}(\tau)$. In particular,

$$
C D F_{S L 2}(\tau)=\Phi\left\{\frac{\mu_{T S L 2}(\tau)-\mu_{F S L 2}}{\left[\sigma_{F S L 2}^{2}+\sigma_{T S L 2}^{2}(\tau)\right]^{1 / 2}}\right\}
$$

for $F S L 2(T) \sim N\left(\mu_{F S L 1}, \sigma_{F S L 1}\right)$ and $T S L 2(T \mid \tau) \sim N\left[\mu_{T S L 2}(\tau), \sigma_{T S L 2}(\tau)\right]$

$$
C D F_{S L 2}(\tau)=\Phi\left\{\frac{\ln \left[m_{T S L 2}(\tau) / m_{F S L 2}\right]}{\left[\beta_{F S L 2}^{2}+\beta_{T S L 2}^{2}(\tau)\right]^{1 / 2}}\right\}
$$

for $F S L 2(T) \sim L N\left(m_{F S L 2}, \beta_{F S L 2}\right)$ and $T S L 2(T \mid \tau) \sim L N\left[m_{T S L 2}(\tau), \beta_{T S L 2}(\tau)\right]$;

$$
C D F_{W L}(\tau)=\Phi\left\{\frac{\mu_{T W L}(\tau)-\mu_{F W L}}{\left[\sigma_{F W L}^{2}+\sigma_{T W L}^{2}(\tau)\right]^{1 / 2}}\right\}
$$

for $F W L(T) \sim N\left(\mu_{F W L}, \sigma_{F W L}\right)$ and $\left.T W L(T \mid \tau) \sim N \mu_{T W L}(\tau), \sigma_{T W L}(\tau)\right] ;$ and

$$
C D F_{W L}(\tau)=\Phi\left\{\frac{\ln \left[m_{T W L}(\tau) / m_{F W L}\right]}{\left[\beta_{F W L}^{2}+\beta_{T W L}^{2}(\tau)\right]^{1 / 2}}\right\}
$$

for $F W L(T) \sim L N\left(m_{F W L}, \boldsymbol{\beta}_{F W L}\right)$ and $T W L(T \mid \tau) \sim L N\left[m_{T W L}(\tau), \beta_{T W L}(\tau)\right]$.

The final representation for $\overline{p F}(t)$ in Eq. (7.1) is used in the determination of the probability of loss of assured safety. The form of this representation in the program P-RACE developed by Bohn (App. II) uses calculated values for the derivative $\mathrm{d} C D F_{W L}(\tau) / \mathrm{d} \tau$ based on the forms of $C D F_{W L}(\tau)$ in Eqs. (7.26) and (7.27). Two options are allowed for the specification of $F W L(T)$ and $T W L(T \mid \tau)$ in the input to P-RACE: (i) $F W L(T) \sim N\left(\mu_{F W L}, \sigma_{F W L}\right)$ and 
$T W L(T \mid \tau) \sim N\left[\mu_{T W L}(\tau), \operatorname{Cov}_{T W L} \mu_{T W L}(\tau)\right]$, where $\operatorname{Cov}_{T W L}=\sigma_{T W L}(\tau) / \mu_{T W L}(\tau)$ is a time independent coefficient of variation for WL temperature, and (ii) $F W L(T) \sim L N\left(m_{F W L}, \beta_{F W L}\right)$ and $T W L(T \mid \tau) \sim L N\left[m_{T W L}(\tau), \beta_{T W L}\right]$. Further, $\mu_{T W L}(\tau)$ and $m_{T W L}(\tau)$ are restricted to a limited number of simple algebraic forms. An important distinction between the two options, and the source of the error in P-RACE as described in conjunction with Eqs. (7.42) and (7.43) in Sect. 7.2, is that $\sigma_{T W L}(\tau)=\operatorname{Cov}_{T W L} \mu_{T W L}(\tau)$ is time dependent in the specification of normal distributions for WL temperature and $\beta_{T W L}$ is time independent in the specification of lognormal distributions for WL temperature. Analogous distribution options are allowed for the two SLs.

The determination of $\mathrm{d} C D F_{W L}(\tau) / \mathrm{d} \tau$ for $F W L(T) \sim N\left(\mu_{F W L}, \sigma_{F W L}\right)$ and $T W L(T \mid \tau) \sim N\left[\mu_{T W L}(\tau), \operatorname{Cov}_{T W L}\right.$ $\left.\mu_{T W L}(\tau)\right]$ is considered first. Specifically,

$$
\begin{aligned}
{\mathrm{d} C D F_{W L}(\tau) / \mathrm{d} \tau} & =\frac{\mathrm{d}}{\mathrm{d} \tau} \Phi[F(\tau)] \\
& =\frac{d}{d \tau}\left[\int_{-\infty}^{F(\tau)}(1 / \sqrt{2 \pi}) \exp \left(-v^{2} / 2\right) \mathrm{d} v\right] \\
& =(1 / \sqrt{2 \pi}) \exp \left[-F^{2}(\tau) / 2\right] \mathrm{d} F(\tau) / \mathrm{d} \tau
\end{aligned}
$$

where

$$
F(\tau)=\frac{\mu_{T W L}(\tau)-\mu_{F W L}}{\left[\sigma_{F W L}^{2}+\operatorname{Cov}_{T W L}^{2} \mu_{T W L}^{2}(\tau)\right]^{1 / 2}}
$$

Further, from the derivative identity $(f / g)^{\prime}=\left(f^{\prime} g-f g^{\prime}\right) / \mathrm{g}^{2}$,

$$
\begin{aligned}
\mathrm{d} F(\tau) / \mathrm{d} \tau= & \left(\left[\mu_{T W L}(\tau)-\mu_{F W L}\right]^{\prime}\left[\sigma_{F W L}^{2}+\operatorname{Cov}_{T W L}^{2} \mu_{T W L}^{2}(\tau)\right]^{1 / 2}\right. \\
& \left.-\left\{\mu_{T W L}(\tau)-\mu_{F W L}\right\}\left\{\left[\sigma_{F W L}^{2}+\operatorname{Cov}_{T W L}^{2} \mu_{T W L}^{2}(\tau)\right]^{1 / 2}\right\}\right) \\
& \cdot\left(\sigma_{F W L}^{2}+\operatorname{Cov}_{T W L}^{2} \mu_{T W L}^{2}(\tau)\right)^{-1} \\
= & \left(\mu_{T W L}^{\prime}(\tau)\left[\sigma_{F W L}^{2}+\operatorname{Cov}_{T W L}^{2} \mu_{T W L}^{2}(\tau)\right]^{1 / 2}\right. \\
& \left.-\left\{\mu_{T W L}(\tau)-\mu_{F W L}\right\}\left\{\left[\sigma_{F W L}^{2}+\operatorname{Cov}_{T W L}^{2} \mu_{T W L}^{2}(\tau)\right]^{-1 / 2}\right\} \operatorname{Cov}_{T W L}^{2} \mu_{T W L}(\tau) \mu_{T W L}^{\prime}(\tau)\right) \\
& \cdot\left(\sigma_{F W L}^{2}+\operatorname{Cov}_{T W L}^{2} \mu_{T W L}^{2}(\tau)\right)^{-1}
\end{aligned}
$$




$$
\begin{aligned}
= & \left(1-\left[\mu_{T W L}(\tau)-\mu_{F W L}\right]\left[\sigma_{F W L}^{2}+\operatorname{Cov}_{T W L}^{2} \mu_{T W L}^{2}(\tau)\right]^{-1} \operatorname{Cov}_{T W L}^{2} \mu_{T W L}(\tau)\right) \\
& \cdot\left(\sigma_{F W L}^{2}+\operatorname{Cov}_{T W L}^{2} \mu_{T W L}^{2}(\tau)\right)^{-1 / 2} \mu_{T W L}^{\prime}(\tau) .
\end{aligned}
$$

The complicated form for $\mathrm{d} C D F_{W L}(\tau) / \mathrm{d} \tau$ results from the time dependence of $\sigma_{T W L}(\tau)=\operatorname{Cov}_{T W L} \mu_{T W L}(\tau)$ in the denominator of the expression defining $F(\tau)$.

The determination of $\mathrm{d} C D F_{W L}(\tau) / \mathrm{d} \tau$ for $F W L(T) \sim L N\left(m_{F W L}, \beta_{F W L}\right)$ and $T W L(T \mid \tau) \sim L N\left[m_{T W L}(\tau), \beta_{T W L}\right]$ is now considered. In this case, the representation for $\mathrm{d} C D F_{W L}(\tau) / \mathrm{d} \tau$ is the same as in Eq. (7.28) with the change that $F(\tau)$ is now given by

$$
F(\tau)=\frac{\ln \left[m_{T W L}(\tau) / m_{F W L}\right]}{\left[\beta_{F W L}^{2}+\beta_{T W L}^{2}\right]^{1 / 2}}
$$

In turn,

$$
\mathrm{d} F(\tau) / \mathrm{d} \tau=\frac{m_{F W L} m_{T W L}^{\prime}(\tau)}{m_{T W L}(\tau)\left[\beta_{F W L}^{2}+\beta_{T W L}^{2}\right]^{1 / 2}} .
$$

This form for $\mathrm{d} F(\tau) / \mathrm{d} \tau$ is simpler than the form in Eq. (7.30) because the denominator in the representation for $F(\tau)$ in Eq. (7.31) is a constant.

A summary of the representations used for $\overline{p F}(t)$ in Bohn's development for one WL and two SLs is provided in Table 7.1. When $t$ and the associated rise in temperature are sufficiently large to assure that the WL has failed, $\overrightarrow{p F}(t)$ is equal to the expected value for probability of loss of assured safety. Although the distributions used with a given link must be either normal or lognormal, different distribution types can be used for different links. An analogous representation for $\overline{p F}(t)$ holds for one WL and and arbitrary number of SLs.

\subsection{Results for Bohn's Test Problems}

Bohn presents three test problems (Table 7.2). The corresponding failure probabilities $\overline{p F}(80)$ at $t=80 \mathrm{~s}$ are calculated with the P-RACE program using the representation for $\overline{p F}(t)$ in Table 7.1.

The representation for $p F(t)$ in Sect. 2 can be used to evaluate the failure probabilities for the test problems that involve one WL and one SL, and the representation in Sect. 4 can be used to evaluate the failure probabilities for the test problems that involve one WL and two SLs. As developed in Sects. 2 and $4, p F(t)$ is for fixed time temperature curves for the individual links. The Bohn development involves calculating an expected value $\overline{p F}(t)$ 

two SLs

$$
\overline{p F}(t)=\int_{0}^{t} C D F_{S L 1}(\tau) C D F_{S L 2}(\tau)\left[\mathrm{d} C D F_{W L}(\tau) / \mathrm{d} \tau\right] \mathrm{d} \tau
$$

where

$$
\begin{aligned}
C D F_{S L 1}(\tau)=\Phi & \left\{\frac{\mu_{T S L 1}(\tau)-\mu_{F S L 1}}{\left[\sigma_{F S L 1}^{2}+\operatorname{Cov}_{T S L 1}^{2} \mu_{T S L 1}^{2}(\tau)\right]^{1 / 2}}\right\} \\
C D F_{S L 2}(\tau)=\Phi & \left\{\frac{\mu_{T S L 2}(\tau)-\mu_{F S L 2}}{\left[\sigma_{F S L 2}^{2}+C o v_{T S L 2}^{2} \mu_{T S L 2}^{2}(\tau)\right]^{1 / 2}}\right\} \\
\mathrm{d} C D F_{W L}(\tau) / \mathrm{d} \tau= & \left\{\frac{\mu_{T W L}^{\prime}(\tau)}{\sqrt{2 \pi}\left[\sigma_{F W L}^{2}+C o v_{T W L}^{2} \mu_{T W L}^{2}(\tau)\right]^{1 / 2}}\right\}\left\{\exp \left[\frac{-\left[\mu_{T W L}(\tau)-\mu_{F W L}\right]^{2}}{2\left[\sigma_{F W L}^{2}+C o v_{T W L}^{2} \mu_{T W L}^{2}(\tau)\right]}\right]\right\} \\
& \cdot\left\{1-\left[\mu_{T W L}(\tau)-\mu_{F W L}\right]\left[\sigma_{F W L}^{2}+\operatorname{Cov}_{T W L}^{2} \mu_{T W L}^{2}(\tau)\right]^{-1} \operatorname{Cov}_{T W L}^{2} \mu_{T W L}(\tau)\right\}
\end{aligned}
$$

for the specification of normal distributions (i.e., $F W L(T) \sim N\left(\mu_{F W L}, \sigma_{F W L}\right), T W L(T \mid \tau) \sim N\left[\mu_{T W L}(\tau), \operatorname{Cov}_{T W L}\right.$ $\left.\mu_{T W L}(\tau)\right]$, and $F S L 1(T), F S L 2(T), T S L 1(T \mid \tau)$ and $\operatorname{TSL} 2(T \mid \tau)$ are defined similarly), and

$$
\begin{aligned}
& C D F_{S L 1}(\tau)=\Phi\left\{\frac{\ln \left[m_{T S L 1}(\tau) / m_{F S L 1}\right]}{\left[\beta_{F S L 1}^{2}+\beta_{T S L 1}^{2}\right]^{1 / 2}}\right\} \\
& C D F_{S L 2}(\tau)=\Phi\left\{\frac{\ln \left[m_{T S L 2}(\tau) / m_{F S L 2}\right]}{\left[\beta_{F S L 2}^{2}+\beta_{T S L 2}^{2}\right]^{1 / 2}}\right\} \\
& \mathrm{dCDF} F_{W L}(\tau) / \mathrm{d} \tau=\left\{\frac{m_{F W L} m_{T W L}^{\prime}(\tau)}{m_{T W L}(\tau) \sqrt{2 \pi}\left[\beta_{F W L}^{2}+\beta_{T W L}^{2}\right]^{1 / 2}}\right\}\left\{\exp \left[\frac{-\left\{\ln \left[m_{T W L}(\tau) / m_{F W L}\right]\right\}^{2}}{2\left[\beta_{F W L}^{2}+\beta_{T W L}^{2}\right]}\right]\right\}
\end{aligned}
$$

for the specification of lognormal distributions (i.e., $F W L(T) \sim L N\left(m_{F W L}, \beta_{F W L}\right), T W L(T \mid \tau) \sim L N\left[m_{T W L}(\tau)\right.$, $\left.\beta_{T W L}\right]$, and $F S L 1(T), F S L 2(T), T S L 1(T \mid \tau)$, and $T S L 2(T \mid \tau)$ are defined similarly).

for $p F(t)$ with this expectation taken over distributions for possible time-temperature curves characterized by the density functions $T W L(T \mid \tau), T S L 1(T \mid \tau)$ and $T S L 2(T \mid \tau)$. The preceding density functions can be thought of as defining (i) distributions of temperature curves of the form

$$
T M P W L\left(\tau \mid n_{T W L}\right)=\mu_{T W L}(\tau)+n_{T W L} \operatorname{Cov}_{T W L} \mu_{T W L}(\tau)
$$


Table 7.2. Three Test Problems Developed by Bohn for Use with P-RACE Program for Determination of Probability of Loss of Assured Safety (App. I)

\section{Problem 1}

One WL, One SL:

$F W L(T) \sim L N\left(m_{F W L}, \boldsymbol{\beta}_{F W L}\right)=L N\left(350^{\circ} \mathrm{F}, 0.4\right)$

$\operatorname{FSL1}(T) \sim L N\left(m_{F S L 1}, \beta_{F S L 1}\right)=L N\left(900^{\circ} \mathrm{F}, 0.4\right)$

$T W L(T \mid \tau) \sim L N\left[m_{T W L}(\tau), \beta_{T W L}\right]=L N\left[\left(70^{\circ} \mathrm{F} / \mathrm{s}\right) \tau, 0.3\right], 1 \leq \tau \leq 80 \mathrm{~s}$

$T S L 1(T \mid \tau) \sim L N\left[m_{T S L 1}(\tau), \beta_{T S L 1}\right]=L N\left[\left(60^{\circ} \mathrm{F} / \mathrm{s}\right) \tau, 0.3\right], 1 \leq \tau \leq 80 \mathrm{~s}$

One WL, Two SLs:

$F W L(T) \sim L N\left(m_{F W L}, \beta_{F W L}\right)=L N\left(350^{\circ} \mathrm{F}, 0.4\right)$

$\operatorname{FSL1}(T) \sim L N\left(m_{F S L 1}, \beta_{F S L 1}\right)=L N\left(900^{\circ} \mathrm{F}, 0.4\right)$

$F S L 2(T) \sim L N\left(m_{F S L 2}, \beta_{F S L 2}\right)=L N\left(900^{\circ} \mathrm{F}, 0.4\right)$

$T W L(T \mid \tau) \sim L N\left[m_{T W L}(\tau), \beta_{T W L}\right]=L N\left[\left(70^{\circ} \mathrm{F} / \mathrm{s}\right) \tau, 0.3\right], 1 \leq \tau \leq 80 \mathrm{~s}$

$\left.T S L 1(T \mid \tau) \sim L N\left[m_{T S L 1}(\tau), \beta_{T S L 1}\right]=L N\left(60^{\circ} \mathrm{F} / \mathrm{s}\right) \tau, 0.3\right], 1 \leq \tau \leq 80 \mathrm{~s}$

$T S L 2(T \mid \tau) \sim L N\left[m_{T S L 2}(\tau), \beta_{T S L 2}\right]=L N\left[\left(60^{\circ} \mathrm{F} / \mathrm{s}\right) \tau, 0.3\right], 1 \leq \tau \leq 80 \mathrm{~s}$

Correlation between SL failure temperatures: 0

Correlation between SL temperatures: 0

\section{Problem 2}

One WL, Two SLs:

Same as Problem 1 with one WL and two SLs but with a correlation of 1 between SL temperatures.

One WL, Two SLs:

Same as Problem 1 with one WL and two SLs but with a correlation of 1 between SL failure temperatures and a correlation of 1 between SL temperatures

\section{Problem 3}

One WL, One SL:

$F W L(T) \sim N\left(\mu_{F W L}, \sigma_{F W L}\right)=N\left(379.2^{\circ} \mathrm{F}, 146^{\circ} \mathrm{F}\right)$

$F S L 1(T) \sim N\left(\mu_{F S L 1}, \sigma_{F S L 1}\right)=N\left(975^{\circ} \mathrm{F}, 375^{\circ} \mathrm{F}\right)$

$T W L(T \mid \tau) \sim N\left[\mu_{T W L}(\tau), \sigma_{T W L}(\tau)\right]=N\left[\left(73.22^{\circ} \mathrm{F} / \mathrm{s}\right) \tau, 0.31\left(73.22^{\circ} \mathrm{F} / \mathrm{s}\right) \tau\right], 0 \leq \tau \leq 80 \mathrm{~s}$

$\left.T S L 1(T \mid \tau) \sim N \mu_{T S L 1}(\tau), \sigma_{T S L 1}(\tau)\right]=N\left[\left(62.76^{\circ} \mathrm{F} / \mathrm{s}\right) \tau, 0.31\left(62.76^{\circ} \mathrm{F} / \mathrm{s}\right) \tau\right], 0 \leq \tau \leq 80 \mathrm{~s}$ 


$$
\begin{aligned}
& \text { One WL, Two SLs: } \\
& F W L(T) \sim N\left(\mu_{F W L}, \sigma_{F W L}\right)=N\left(379.2^{\circ} \mathrm{F}, 146^{\circ} \mathrm{F}\right) \\
& F S L 1(T) \sim N\left(\mu_{F S L 1}, \sigma_{F S L 1}\right)=N\left(975^{\circ} \mathrm{F}, 375^{\circ} \mathrm{F}\right) \\
& F S L 2(T) \sim N\left(\mu_{F S L 2}, \sigma_{F S L 2}\right)=N\left(975^{\circ} \mathrm{F}, 375^{\circ} \mathrm{F}\right) \\
& T W L(T \mid \tau) \sim N\left[\mu_{T W L}(\tau), \sigma_{T W L}(\tau)\right]=N\left[\left(73.22^{\circ} \mathrm{F} / \mathrm{s}\right) \tau, 0.31\left(73.22^{\circ} \mathrm{F} / \mathrm{s}\right) \tau\right], 0 \leq \tau \leq 80 \mathrm{~s} \\
& T S L 1(T \mid \tau) \sim N\left[\mu_{T S L 1}(\tau), \sigma_{T S L 1}(\tau)\right]=N\left[\left(62.76^{\circ} \mathrm{F} / \mathrm{s}\right) \tau, 0.31\left(62.76^{\circ} \mathrm{F} / \mathrm{s}\right) \tau\right], 0 \leq \tau \leq 80 \mathrm{~s} \\
& T S L 2(T \mid \tau) \sim N\left[\mu_{T S L 2}(\tau), \sigma_{T S L 2}(\tau)\right]=N\left[\left(62.76^{\circ} \mathrm{F} / \mathrm{s}\right) \tau, 0.31\left(62.76^{\circ} \mathrm{F} / \mathrm{s}\right) \tau\right], 0 \leq \tau \leq 80 \mathrm{~s}
\end{aligned}
$$

Correlation between SL failure temperatures: 0

Correlation between SL temperatures: 0

$$
\begin{aligned}
& \operatorname{TMPSL1}\left(\tau \mid n_{T S L 1}\right)=\mu_{T S L 1}(\tau)+n_{T S L 1} \operatorname{Cov}_{T S L 1} \mu_{T S L 1}(\tau) \\
& \operatorname{TMPSL2}\left(\tau \mid n_{T S L 2}\right)=\mu_{T S L 2}(\tau)+n_{T S L 2} \operatorname{Cov}_{T S L 2} \mu_{T S L 2}(\tau)
\end{aligned}
$$

when $T W L(T \mid \tau), T S L 1(T \mid \tau)$ and $T S L 2(T \mid \tau)$ correspond to normal distributions with $n_{T W L}, n_{T S L 1}$ and $n_{T S L 2}$ being standard normal variables (i.e., $n_{T W L} \sim N(0,1), n_{T S L 1} \sim N(0,1), n_{T S L 2} \sim N(0,1)$ ) and (ii) distributions of temperature curves of the form

$$
\begin{aligned}
& T M P W L\left(\tau \mid n_{T W L}\right)=m_{T W L}(\tau) \exp \left(n_{T W L} \beta_{T W L}\right) \\
& \operatorname{TMPSL1}\left(\tau \mid n_{T S L 1}\right)=m_{T S L 1}(\tau) \exp \left(n_{T S L 1} \beta_{T S L 1}\right) \\
& T M P S L 2\left(\tau \mid n_{T S L 2}\right)=m_{T S L 2}(\tau) \exp \left(n_{T S L 2} \beta_{T S L 2}\right)
\end{aligned}
$$

when $T W L(T \mid \tau), T S L 1(T \mid \tau)$ and $T S L 2(T \mid \tau)$ correspond to lognormal distributions with $n_{T W L}, n_{T S L 1}$ and $n_{T S L 2}$ again being standard normal variables.

If one WL and one SL are under consideration, then $\overline{p F}(t)$ can be approximated by

$$
\overline{p F}(t) \cong \sum_{i=1}^{n} p F\left(t \mid n_{T W L, i}, n_{T S L 1, i}\right) / n
$$

where $n_{T W L, i}$ and $n_{T S L 1, i}$ are sampled randomly from $N(0,1)$ and $p F\left(t \mid n_{T W L, i}, n_{T S L 1, i}\right)$ corresponds to $p F(t)$ evaluated with the temperature curves associated with $n_{T W L, i}$ and $n_{T S L 1, i}$ as indicated in Eqs. (7.33) - (7.38). If one WL and two SLs are under consideration, then $\overline{p F}(t)$ is given by the analogous approximation 


$$
\overline{p F}(t) \cong \sum_{i=1}^{n} p F\left(t \mid n_{T W L, i}, n_{T S L 1, i}, n_{T S L 2, i}\right) / n
$$

Problem 2 (Table 7.2) involves unit correlations between failure temperatures and also between the time-dependent temperatures for the two SLs. The implementation of these correlations is trivial and simply involves using the same failure temperature for both SLs when a correlation of 1 is specified for the failure temperature and similarly using the same time-dependent temperature curve for both SLs when a correlation of 1 is specified for timedependent temperature.

The results obtained from P-RACE with the representation for $\overline{p F}(t)$ in Table 7.1 and the results obtained with approximation procedures in Eqs. (7.39) and (7.40) based on evaluating individual time temperature curves agree for Problems 1 and 2 but disagree for Problem 3 (Table 7.3).

The reason for the disagreement for Problem 3 was not apparent initially. A checking of both implementations did not reveal any errors. However, all four numerical procedures for the evaluation of $p F\left(t \mid n_{T W L, i}, n_{T S L 1, i}, n_{T S L 2, i}\right)$ (i.e., two quadrature procedures and two sampling-based procedures) yielded the same results for Problem 3 . This suggested the problem might be in the implementation of the calculations to evaluate $\overline{p F}(t)$ as defined in Table 7.1.

Due to the complexity of dealing with normal and lognormal distributions, it is difficult to do hand calculations to verify the correctness of a numerical result. However, a simple test to verify the presence of an error is possible. In particular, if one WL and $n S L$ SLs are involved, all links have the same failure temperature distributions (i.e., $F W L(T), F S L 1(T), F S L 2(T), \ldots$ correspond to the same distribution) and also the same time-dependent temperature distributions (i.e., $T W L(T \mid \tau), T S L 1(T \mid \tau), T S L 2(T \mid \tau), \ldots$ correspond to the same distribution), and the properties of the individual links are independent, then

$$
\begin{aligned}
\overline{p F}(\infty) & =\lim _{t \rightarrow \infty} \int_{0}^{t}[p(\tau)]^{n S L} p^{\prime}(\tau) \mathrm{d} \tau \\
& =\lim _{t \rightarrow \infty}[p(\tau)]^{n S L+1} /\left.(n S L+1)\right|_{0} ^{t} \\
& =1 /(n S L+1),
\end{aligned}
$$

where the problem is assumed to start at time 0 and $p(\tau)$ is the probability that an individual link will fail by time $\tau$ with $p(0)=0$ and $p(\infty)=1$. In particular, $p(\tau)$ is the same for all links from the assumption that the individual links have the same failure temperature distribution and the same time-dependent temperature distributions.

The indicated test with P-RACE did not yield the required convergence of $\overline{p F}(t)$ to $1 / 2,1 / 3,1 / 4, \ldots$ for $n S L=$ $1,2,3, \ldots$ Thus, there had to be a problem in P-RACE. Examination of P-RACE did not reveal any problems. At this point, a rederivation of the results implemented in P-RACE was undertaken. The content of Sect 7.1 is the outcome of this rederivation. 


\section{Table 7.3. Comparison of $\overline{p F}(t)$ Obtained for Test Problems with Different Computational Proce-} dures

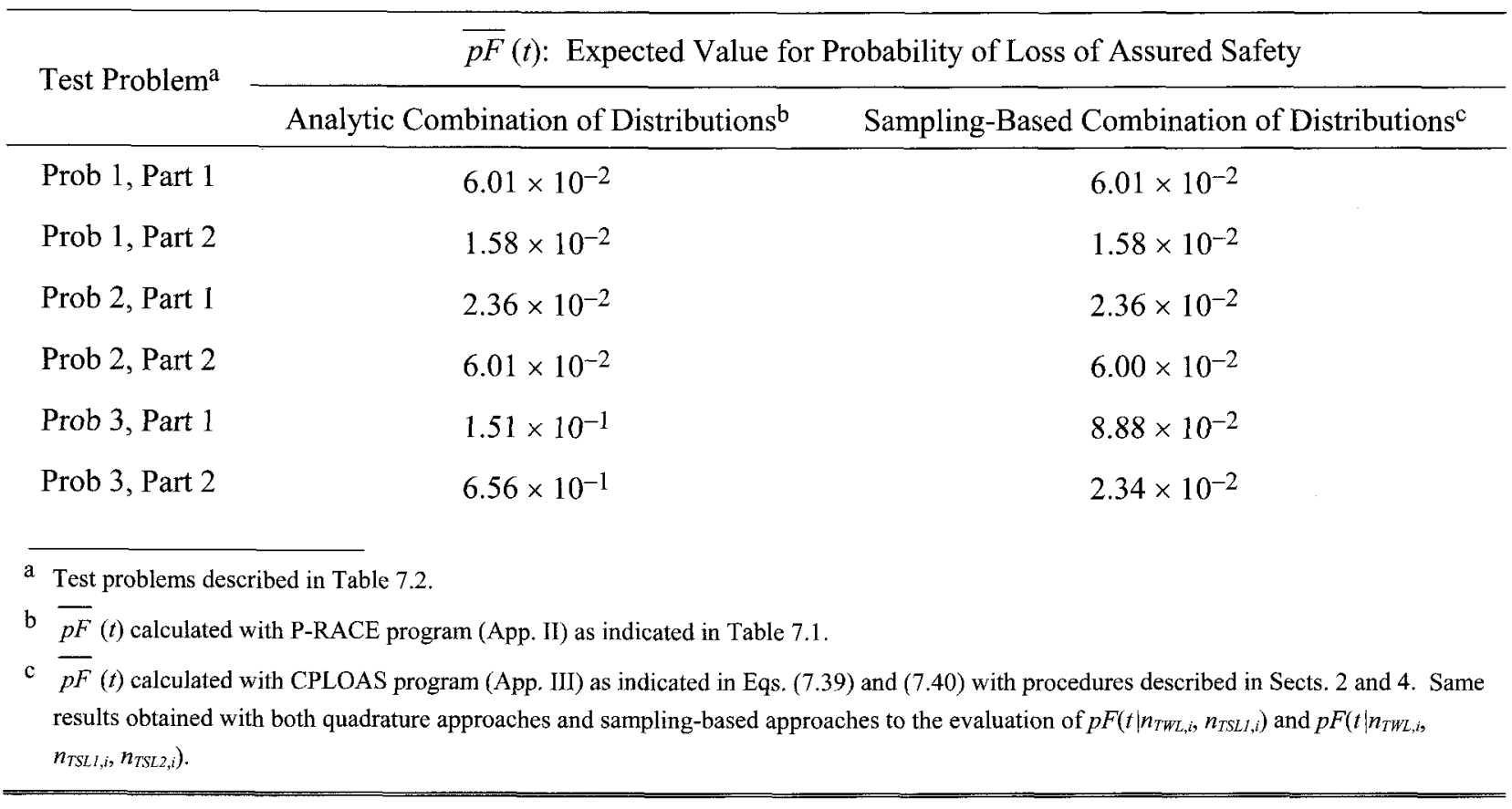

What was discovered in this rederivation was that P-RACE was implemented with

$$
\begin{aligned}
\mathrm{d} F(\tau) / \mathrm{d} \tau & =\mu_{T W L}^{\prime}(\tau) /\left[\sigma_{F W L}^{2}+\sigma_{T W L}^{2}(\tau)\right]^{1 / 2} \\
& =\mu_{T W L}^{\prime}(\tau) /\left[\sigma_{F W L}^{2}+\operatorname{Cov}_{T W L}^{2} \mu_{T W L}^{2}(\tau)\right]^{1 / 2}
\end{aligned}
$$

when $\left.T W L(T \mid \tau) \sim N \mu_{T W L}(\tau), \operatorname{Cov}_{T W L} \mu_{T W L}(\tau)\right]$ rather than with the correct value for $\mathrm{d} F(\tau) / \mathrm{d} \tau$ in Eq. (7.30). The derivative

$$
\mathrm{d} F(\tau) / \mathrm{d} \tau=\mu_{T W L}^{\prime}(\tau) /\left(\sigma_{F W L}^{2}+\sigma_{T W L}^{2}\right)^{1 / 2}
$$

is correct where $\sigma_{T W L}$ is a constant rather than a function of time (i.e., $\sigma_{T W L}(\tau)=\operatorname{Cov}_{T W L} \mu_{T W L}(\tau)$ ). This is an easy error to make. The defining relationship for $\mathrm{d} F(\tau) / \mathrm{d} \tau$ was probably originally and correctly derived with the assumption of constant values for $\sigma_{F W L}$ and $\sigma_{T W L}$; then at some later time it is likely that a decision was made to make $\sigma_{T W L}$ a function of time without recollection of the assumption of a constant value for $\sigma_{T W L}$ in the derivation of $\mathrm{d} F(\tau) / \mathrm{d} \tau$. Once the correction to the definition of $\mathrm{d} F(\tau) / \mathrm{d} \tau$ was made, P-RACE produced the same values for $\overline{p F}(t)$ as given in Table 7.3 for the sampling-based approach.

The existence of a problem in P-RACE for calculations carried out with normal distributions had been suspected for a long time. For this reason, decision-supporting calculations were not carried out with P-RACE for nor- 
mal distributions. However, the exact nature of this problem was not recognized until after the derivations in Sect. 7.1 were developed. 


\section{Summary}

The probability of loss of assured safety (PLOAS) in WL/SL systems under fire conditions is investigated. The indicated probability refers to the failure of the WL system to deactivate the overall systems before failure of the SL system and derives from variability (i.e., aleatory uncertainty) in the temperatures at which the individual links will fail.

Formal developments of the probability that the WL system fails to deactivate the overall system before failure of the SL system (i.e., PLOAS) are presented for four WL/SL configurations: (i) one WL, one SL (Sect. 2), (ii) multiple WLs, multiple SLs with failure of any SL before any WL constituting failure of the safety system (Sect. 3), (iii) multiple WLs, multiple SLs with failure of all SLs before any WL constituting failure of the safety system (Sect. 4), and (iv) multiple WLs, multiple SLs and multiple sublinks in each SL with failure of a sublink constituting failure of the associated SL and failure of all SLs before failure of any WL constituting failure of the safety system (Sect. 5). The indicated probabilities derive from time-dependent temperatures in the WL/SL system and variability in the temperatures at which the individual components of this system fail and are formally defined as multidimensional integrals.

Numerical procedures based on quadrature (i.e., trapezoidal rule, Simpson's rule) and also on Monte Carlo techniques (i.e., simple random sampling, importance sampling) are described and illustrated for the evaluation of these integrals. Further, the FORTRAN program CPLOAS has been written to implement the indicated numerical procedures for the calculation of PLOAS (App. III). The quadrature-based procedures are numerically much more efficient (i.e., require fewer function evaluations) than the Monte Carlo techniques. However, the Monte Carlo techniques are useful in that they provide an independent verification of the correctness of the conceptual and computational implementation of the quadrature-based procedures.

Example uncertainty and sensitivity analyses for PLOAS involving the representation of uncertainty (i.e., epistemic uncertainty) with probability theory and also with evidence theory are presented (Sect. 6). A sampling-based approach for the propagation of uncertainty is used for uncertainty representations based on probability and also for uncertainty representations based on evidence theory. Further, a computationally efficient procedure for the determination of complementary cumulative belief functions and complementary cumulative plausibility functions is introduced.

A careful derivation of a numerical procedure originally introduced by M.P. Bohn (App. I) for the determination of PLOAS is presented (Sect. 7). This derivation facilitated the identification of an error in the P-RACE program for the calculation PLOAS. The existence of some type of error in P-RACE had been previously suspected but its exact nature was not known. The indicated derivation led to the identification of this error and also to a useful result/procedure for use in the verification of PLOAS calculations (Eq. (7.41)). After correction of this error, 
P-RACE and CPLOAS produce the same values for PLOAS for three test problems defined by Bohn for use with P-RACE. 


\section{References}

1. Andrews, J.D. and T.R. Moss. 2002. Reliability and Risk Assessment (2nd Ed.). New York, NY: ASME Press.

2. Melchers, R.E. 1998. Structural Reliability Analysis and Prediction (2nd Ed.). New York, NY: John Wiley \& Sons.

3. Schuëller, G.I. 1998. "Structural Reliability-Recent Advances," M. Shinozuka and Y.K. Wen (eds), Structural Safety and Reliability. pp. 3-35.

4. Kovalenko, I.N., N.Y. Kuznetsov, and P.A. Pegg. 1997. Mathematical Theory of Reliability of Time Dependent Systems with Practical Applications. New York, NY: John Wiley \& Sons.

5. Upshakov, I.A. and R.A. Harrison (eds). 1994. Handbook of Reliability. New York, NY: John Wiley \& Sons.

6. Birolini, A. 1994. Quality and Reliability of Technical Systems. New York, NY: Springer-Verlag.

7. Bolotin, V.V. 1969. Statistical Methods in Structural Mechanics. San Francisco, CA: Holden-Day.

8. Helton, J.C. 1997. "Uncertainty and Sensitivity Analysis in the Presence of Stochastic and Subjective Uncertainty," Journal of Statistical Computation and Simulation. Vol. 57, no. 1-4, pp. 3-76.

9. Paté-Cornell, M.E. 1996. "Uncertainties in Risk Analysis: Six Levels of Treatment," Reliability Engineering and System Safety. Vol. 54, no. 2-3, pp. 95-111.

10. Helton, J.C. 1994. "Treatment of Uncertainty in Performance Assessments for Complex Systems," Risk Analysis. Vol. 14, no. 4, pp. 483-511.

11. Hoffman, F.O. and J.S. Hammonds. 1994. "Propagation of Uncertainty in Risk Assessments: The Need to Distinguish Between Uncertainty Due to Lack of Knowledge and Uncertainty Due to Variability," Risk Analysis. Vol. 14, no. 5, pp. 707-712.

12. Crowder, M.J. 2001. Classical Competing Risks. Boca Raton, FL: Chapman \& Hall/CRC.

13. David, H.A. and M.L. Moeschberger. 1978. The Theory of Competing Risks. London: Griffin.

14. Seal, H.L. 1977. "Studies in the History of Probability and Statistics, XXXV. Multiple Decrements or Competing Risks," Biometrika. Vol. 64, pp. 429-439. 
15. Gail, M. 1975. "Review and Critique of Some Models Used in Competing Risk Analysis," Biometrics. Vol. 31 , no. 1, pp. 209-222.

16. Bartle, R.G. 1975. The Elements of Real Analysis (2nd Ed.). New York, NY: John Wiley \& Sons.

17. K. Rektorys (Ed.). 1969. Survey of Applicable Mathematics. Cambridge, MA: The M.I.T. Press.

18. Zhang, S. and J. Jin. 1996. Computation of Special Functions. New York, NY: John Wiley \& Sons.

19. Press, W.H., S.A. Teukolsky, W.T. Vetterling, and B.P. Flannery. 1992. Numerical Recipes in FORTRAN: The Art of Scientific Computing. 2nd ed. Cambridge; New York: Cambridge University Press.

20. Krommer, A.R. and C.W. Ueberhuber. 1998. Computational Integration. Philadelphia, PA: SIAM.

21. Evans, M. and T. Swartz. 2000. Approximating Integrals via Monte Carlo and Deterministic Methods. Oxford, New York: Oxford University Press.

22. Hurtado, J.E. and A.H. Barbat. 1998. "Monte Carlo Techniques in Computational Stochastic Mechanics," Archives of Computational Methods in Engineering. Vol. 5, no. 1, pp. 3-330.

23. Nicola, V.F., P. Shahabuddin, and M.K. Nakayama. 2001. "Techniques for Fast Simulation of Models of Highly Dependable Systems," IEEE Transactions on Reliability. Vol. 50, no. 3, pp. 246-264.

24. Owen, A. and Y. Zhou. 2000. "Safe and Effective Importance Sampling," Journal of American Statistical Association. Vol. 95, no. 449, pp. 135-143.

25. Heidelberger, P. 1995. "Fast Simulation of Rare Events in Queueing and Reliability Models," ACM Transactions on Modeling and Computer Simulation. Vol. 5, no. 1, pp. 43-85.

26. Shahabuddin, P. 1994. "Importance Sampling for the Simulation of Highly Reliable Markovian Systems," Management Science. Vol. 40, no. 3, pp. 333-352.

27. Goyal, A., P. Shahabuddin, P. Heidelberger, V.F. Nicola, and P.W. Glynn. 1992. "A Unified Framework for Simulating Markovian Models of Highly Dependable Systems," IEEE Transactions on Computers. Vol. 41, no. 1, pp. 36-51.

28. Melchers, R.E. 1990. “Search-Based Importance Sampling,” Structural Safety. Vol. 9, no. 2, pp. 117-128.

29. Glynn, P.W. and D.L. Iglehart. 1989. "Importance Sampling for Stochastic Simulations," Management Science. Vol. 35, no. 11, pp. 1367-1392. 
30. Helton, J.C., W.L. Oberkampf, and J.D. Johnson. 2004. "Competing Failure Risk Analysis Using Evidence Theory," Risk Analysis (to appear).

31. Wagner, R.L. 2003. "Science, Uncertainty and Risk: The Problem of Complex Phenomena," APS News. Vol. 12 , no. 1, pp. 8 .

32. Oberkampf, W.L., S.M. DeLand, B.M. Rutherford, K.V. Diegert, and K.F. Alvin. 2002. "Error and Uncertainty in Modeling and Simulation," Reliability Engineering and System Safety. Vol. 75, no. 3, pp. 333-357.

33. NRC (National Research Council). 1994. Science and Judgment in Risk Assessment, Washington, DC: National Academy Press.

34. NRC (National Research Council). 1993. Issues in Risk Assessment. Washington, DC: National Academy Press.

35. Helton, J.C. and D.E. Burmaster. 1996. "Guest Editorial: Treatment of Aleatory and Epistemic Uncertainty in Performance Assessments for Complex Systems," Reliability Engineering and System Safety. Vol. 54, no. 2-3, pp. 91-94.

36. Parry, G.W. and P.W. Winter. 1981. "Characterization and Evaluation of Uncertainty in Probabilistic Risk Analysis," Nuclear Safety. Vol. 22, no. 1, pp. 28-42.

37. Apostolakis, G. 1990. "The Concept of Probability in Safety Assessments of Technological Systems," Science. Vol. 250, no. 4986, pp. 1359-1364.

38. Helton, J.C. 1993. "Uncertainty and Sensitivity Analysis Techniques for Use in Performance Assessment for Radioactive Waste Disposal," Reliability Engineering and System Safety. Vol. 42, no. 2-3, pp. 327-367.

39. Blower, S.M. and H. Dowlatabadi. 1994. "Sensitivity and Uncertainty Analysis of Complex Models of Disease Transmission: an HIV Model, as an Example," International Statistical Review. Vol. 62, no. 2, pp. 229243.

40. Hamby, D.M. 1994. "A Review of Techniques for Parameter Sensitivity Analysis of Environmental Models," Environmental Monitoring and Assessment. Vol. 32, no. 2, pp. 135-154.

41. Kleijnen, J.P.C. and J.C. Helton. 1999. "Statistical Analyses of Scatterplots to Identify Important Factors in Large-Scale Simulations, 1: Review and Comparison of Techniques," Reliability Engineering and System Safety. Vol. 65, no. 2, pp. 147-185.

42. Saltelli, A., K. Chan, and E.M. Scott. 2000. Sensitivity Analysis. New York: Wiley. 
43. Helton, J.C. and F.J. Davis. 2002. "Illustration of Sampling-Based Methods for Uncertainty and Sensitivity Analysis," Risk Analysis. Vol. 22, no. 3, pp. 591-622.

44. Dempster, A.P. 1967. "Upper and Lower Probabilities Induced by a Multivalued Mapping," Annals of Mathematical Statistics. Vol. 38, pp. 325-339.

45. Shafer, G. 1976. A Mathematical Theory of Evidence, Princeton, NJ: Princeton Univ. Press.

46. Wasserman, L. 1988. Belief Functions and Likelihood, Technical Report 420, Pittsburgh, PA: Department of Statistics, Carnegie-Mellon University.

47. Wasserman, L.A. 1990. "Belief Functions and Statistical Inference," The Canadian Journal of Statistics. Vol. 18 , no. 3, pp. 183-196.

48. Guan, J.W. and D.A. Bell. 1991. Evidence Theory and Its Applications, Vols. 1, 2. New York: NorthHolland.

49. Halpern, J.Y. and R. Fagin. 1992. "Two Views of Belief: Belief as Generalized Probability and Belief as Evidence," Artificial Intelligence. Vol. 54, pp. 275-317.

50. Yager, R.R., J. Kacprzyk, and M. Fedrizzi (eds). 1994. Advances in the Dempster-Shafer Theory of Evidence. New York: Wiley.

51. Helton, J.C., J.D. Johnson, and W.L. Oberkampf. 2004. "An Exploration of Alternative Approaches to the Representation of Uncertainty in Model Predictions," Reliability Engineering and System Safety. Vol. 85, no. 1-3, pp. 39-71.

52. Cooke, R.M. 1991. Experts in Uncertainty: Opinion and Subjective Probability in Science. Oxford; New York: Oxford University Press.

53. Meyer, M.A. and J.M. Booker. 1991. Eliciting and Analyzing Expert Judgment: A Practical Guide. New York: Academic Press.

54. Chhibber, S., G. Apostolakis, and D. Okrent. 1992. "A Taxonomy of Issues Related to the Use of Expert Judgments in Probabilistic Safety Studies," Reliability Engineering and System Safety. Vol. 38, no. 1-2, pp. 27-45.

55. Otway, H. and D.V. Winterfeldt. 1992. "Expert Judgement in Risk Analysis and Management: Process, Context, and Pitfalls," Risk Analysis. Vol. 12, no. 1, pp. 83-93. 
56. Thorne, M.C. and M.M.R. Williams. 1992. "A Review of Expert Judgement Techniques with Reference to Nuclear Safety," Progress in Nuclear Safety. Vol. 27, no. 2-3, pp. 83-254.

57. Thorne, M.C. 1993. "The Use of Expert Opinion in Formulating Conceptual Models of Underground Disposal Systems and the Treatment of Associated Bias," Reliability Engineering and System Safety. Vol. 42, no. 2-3, pp. 161-180.

58. Evans, J.S., G.M. Gray, R.L. Sielken Jr., A.E. Smith, C. Valdez-Flores, and J.D. Graham. 1994. "Use of Probabilistic Expert Judgement in Uncertainty Analysis of Carcinogenic Potency," Regulatory Toxicology and Pharmacology. Vol. 20, no. 1, pt. 1, pp. 15-36.

59. Budnitz, R.J., G. Apostolakis, D.M. Boore, L.S. Cluff, K.J. Coppersmith, C.A. Cornell, and P.A. Morris. 1998. "Use of Technical Expert Panels: Applications to Probabilistic Seismic Hazard Analysis," Risk Analysis. Vol. 18 , no. 4, pp. 463-469.

60. Goossens, L.H.J. and F.T. Harper. 1998. "Joint EC/USNRC Expert Judgement Driven Radiological Protection Uncertainty Analysis," Journal of Radiological Protection. Vol. 18, no. 4, pp. 249-264.

61. Siu, N.O. and D.L. Kelly. 1998. "Bayesian Parameter Estimation in Probabilistic Risk Assessment," Reliability Engineering and System Safety. Vol. 62, no. 1-2, pp. 89-116.

62. Goossens, L.H.J., F.T. Harper, B.C.P. Kraan, and H. Metivier. 2000. "Expert Judgement for a Probabilistic Accident Consequence Uncertainty Analysis," Radiation Protection Dosimetry. Vol. 90, no. 3, pp. 295-301.

63. McKay, M. and M. Meyer. 2000. "Critique of and Limitations on the use of Expert Judgements in Accident Consequence Uncertainty Analysis," Radiation Protection Dosimetry. Vol. 90, no. 3, pp. 325-330.

64. Feller, W. 1971. An Introduction to Probability Theory and Its Applications. Vol. 2, 2nd ed. New York: John Wiley \& Sons.

65. McKay, M.D., R.J. Beckman, and W.J. Conover. 1979. "A Comparison of Three Methods for Selecting Values of Input Variables in the Analysis of Output from a Computer Code," Technometrics. Vol. 21, no. 2, pp. 239-245.

66. Iman, R.L. 1992. "Uncertainty and Sensitivity Analysis for Computer Modeling Applications," Reliability Technology - 1992, The Winter Annual Meeting of the American Society of Mechanical Engineers, Anaheim, California, November 8-13, 1992. Eds. T.A. Cruse. Vol. 28, pp. 153-168. New York, NY: American Society of Mechanical Engineers, Aerospace Division. 
67. Joslyn, C. and J.C. Helton. 2002. "Bounds on belief and plausibility of functionality propagated random sets," 2002 Annual Meetings of the North American Fuzzy Information Processing Society, Proceedings, 2729 June 2002. Ed. J. Keller and O. Nasraoui. New Orleans, LA: Piscataway, NJ: IEEE, 2002. 412-417.

68. Joslyn, C. and V. Kreinovich. 2002. Convergence Properties of an Interval Probabilistic Approach to System Reliability Estimation, LA-UR-02-6261. Los Alamos, NM: Los Alamos National Laboratory.

69. Howson, C. and P. Urbach. 1993. Scientific Reasoning: The Bayesian Approach. 2nd ed. Chicago: Open Court.

70. Ayyub, B.M. 2001. Elicitation of Expert Opinions for Uncertainty and Risks, Boca Raton: CRC Press.

71. Ferson, S., V. Kreinovich, L. Ginzburg, D.S. Myers, and K. Sentz. 2003. Constructing Probability Boxes and Dempster Shafer Structures, SAND2003-4015. Albuquerque, NM: Sandia National Laboratories.

72. Dubois, D. and H. Prade. 1992. "On the Combination of Evidence in Various Mathematical Frameworks," Reliability Data Collection and Analysis. Ed. J. Flamm and T. Luisi. Boston, MA: Kluwer Academic Publishers. pp. 213-241.

73. Sentz, K. and S. Ferson. 2002. Combination of Evidence in Dempster-Shafer Theory, SAND2002-0835. Albuquerque, NM: Sandia National Laboratories.

74. Hora, S.C. and R.L. Iman. 1989. "Expert Opinion in Risk Analysis: The NUREG-1150 Methodology," Nuclear Science and Engineering. Vol. 102, no. 4, pp. 323-331.

75. Helton, J.C. and F.J. Davis. 2003. "Latin Hypercube Sampling and the Propagation of Uncertainty in Analyses of Complex Systems," Reliability Engineering and System Safety. Vol. 81, no. 1, pp. 23-69.

76. Iman, R.L. and W.J. Conover. 1979. "The Use of the Rank Transform in Regression," Technometrics. Vol. 21, no. 4, pp. 499-509.

77. Helton, J.C. and F.J. Davis. 2000. "Sampling-Based Methods," Sensitivity Analysis. Ed. A. Saltelli, K. Chan, and E.M. Scott. New York, NY: Wiley. pp. 101-153. 


\title{
Appendix I: An Integral Formulation for Calculating Probabilities of Strong Link/Weak Link Cutsets
}

\author{
M.P. Bohn \\ May 10, 1996 \\ This appendix contains a previously unpublished report by M.P. Bohn on the quantification of the probability of \\ failure of temperature-dependent weak link/strong link systems.
}




\section{Sandia National Laboratories}

Albuquerque, New Mexico 87185-0405

date: May 10, 1996

to: David D. Carlson, MS-0405 (12333)

from: Michael P. Bohn, MS-0405 (12333)

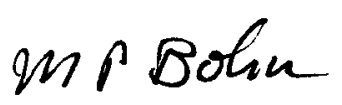

subject: An Integral Formulation for Race Cutset Quantification

Attached is a draft report entitled "An Integral Formulation for Calculating Probabilities of Strong Link/Weak Link Cutsets (Including Uncertainty and Correlation)" which presents equations based on single quadrature for the calculation of race cutset probabilities including random uncertainty, systematic (modeling) uncertainty and arbitrary correlation between the strong link failures.

A computer code (P-RACE) was written to implement this integral formulation, and a Users' Manual is included in the report. The code P-RACE computes either cutset point estimate probabilities or (by manually including modeling uncertainty in the input) the mean failure probability for the cutset.

Finally, this formulation is being extended to compute the full distribution of race cutset failure probability (an example of which is included in the report). The full distributions can be used as input to the TEMAC code in order to calculate the probability distribution of an accident sequence which models loss of assured nuclear detonation safety.

$\mathrm{MPB} / \mathrm{jg}$

Distribution:

MS-0405 Brad S. Altman, 6413

MS-0405 Allan S. Benjamin, 6413

MS-0405 Roger J. Breeding, 12333

MS-0405 Michael A. Dvorack, 12333

MS-0405 Martin K. Fuentes, 12333

MS-0405 Todd R. Jones, 12333

MS-0405 Kevin J. Maloney, 12333

MS-0405 Keri B. Sobolik, 12333

MS-0557 Thomas L. Paez, 2741

Exceptional Service in the National Interest 
MS-0747 Gregory D. Wyss, 6412

MS-0835 Vicente J. Romero, 1513

MS-0633 Paul Demmie, 12333

MS-1175 Martin P. Sherman, 9364

Copy to:

MS-0405 M. P. Bohn, 12333 


\section{AN INTEGRAL FORMULATION FOR CALCULATING PROBABILITIES OF STRONG LINK/WEAK LINK CUTSETS \\ (Including Uncertainty and Correlation)}

by

Michael P. Bohn

May 1996 


\subsection{Introduction}

Certain systems are designed to withstand external environment challenges (loads and temperatures) using a strong link/weak link philosophy to prevent electrical current from being inadvertently passed through the system (the undesired top event). In this design approach, the system employs a number of strong links designed to have a very high threshold of failure (given the external environment challenge) in conjunction with a weak link which is intended to predictably and reliably fail under the external environment challenge. The weak link element is an element of the system which -- if failed -- renders the undesired top event impossible. Thus, if a fault tree of the system is prepared and the minimal cutsets are obtained, one will have (in a properly designed system) several strong link failure events and a single weak link success event in each cutset. (Of course, each cutset may also have failure events not related to the external challenge; e.g., component failures due to human error or unavailabilities due to test and maintenance activities.)

In the following, we will focus on failures resulting from an external thermal environment, and thus assume that temperature-time histories are available for each strong link and the weak link. Further, we assume that a characterization of the failure temperature probability distribution of each strong link and weak link is available. These are shown schematically in Figure 1.

One direct approach which has been used in calculating the probability of such strong link/weak link cutsets is Monte Carlo sampling. In this approach, the temperature-time history of each strong link (and weak link) is combined with the corresponding failure temperature distribution to derive a probability distribution on the time to failure for each element (as shown schematically in Figure 2.) Then, assuming the failure events are independent, random samples of time to failure for each of the strong links and the weak link are generated, and for each set of samples, the question is asked (assuming two strong links and one weak link):

$$
\text { Is } \mathrm{t}_{\mathrm{SL} 1}<\mathrm{t}_{\mathrm{WL}} \text { and } \mathrm{t}_{\mathrm{SL} 2}<\mathrm{t}_{\mathrm{WL}} \text { ? }
$$

After multiple sample sets have been generated, the probability of the cutset is estimated as

$$
\mathrm{P}(\mathrm{SL} 1 * \mathrm{SL} 2 * \overline{W L})=\frac{\text { Number of sets in affirmative }}{\text { Total number of sample sets }}
$$

This approach is quite valid, and (with care) can be extended to consider a wide range of alternative underlying assumptions However, in direct application, the approach has a number of disadvantages:

1) Systematic incorporation of uncertainty in both the time histories , and the failure temperatures is awkward. 


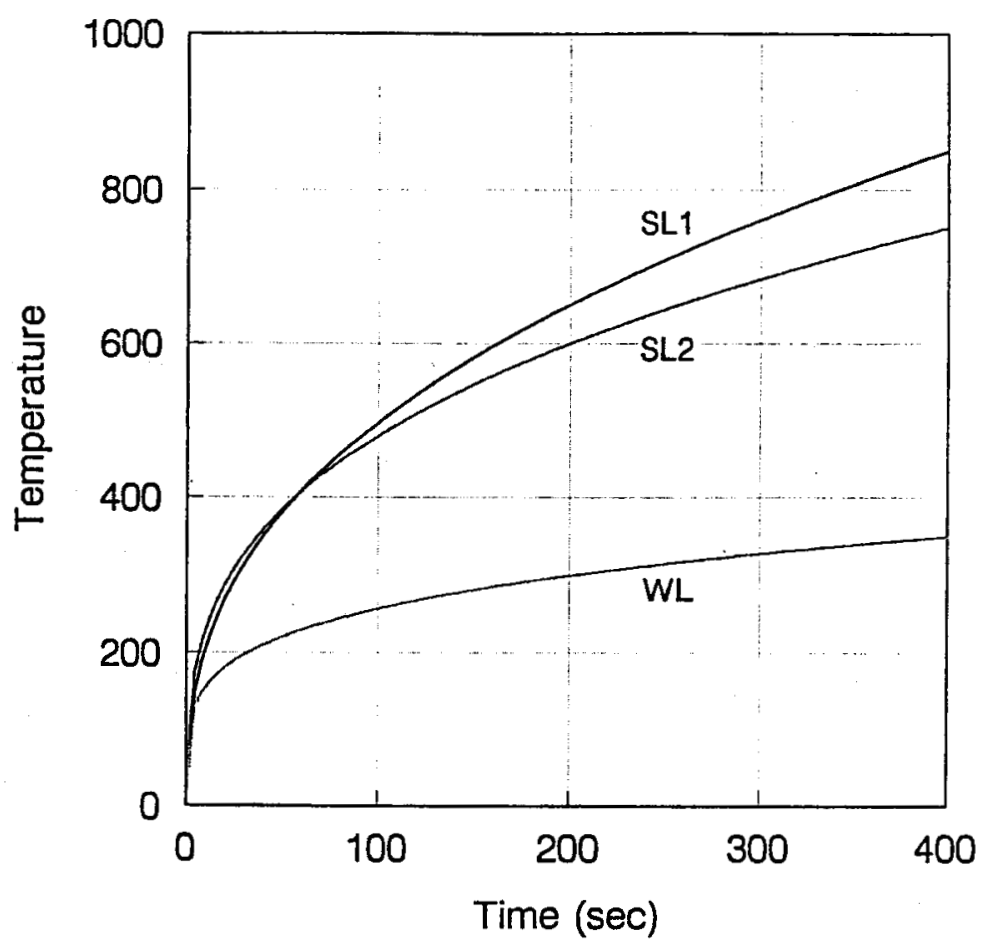

Figure 1 (a) Typical temperature-time histories

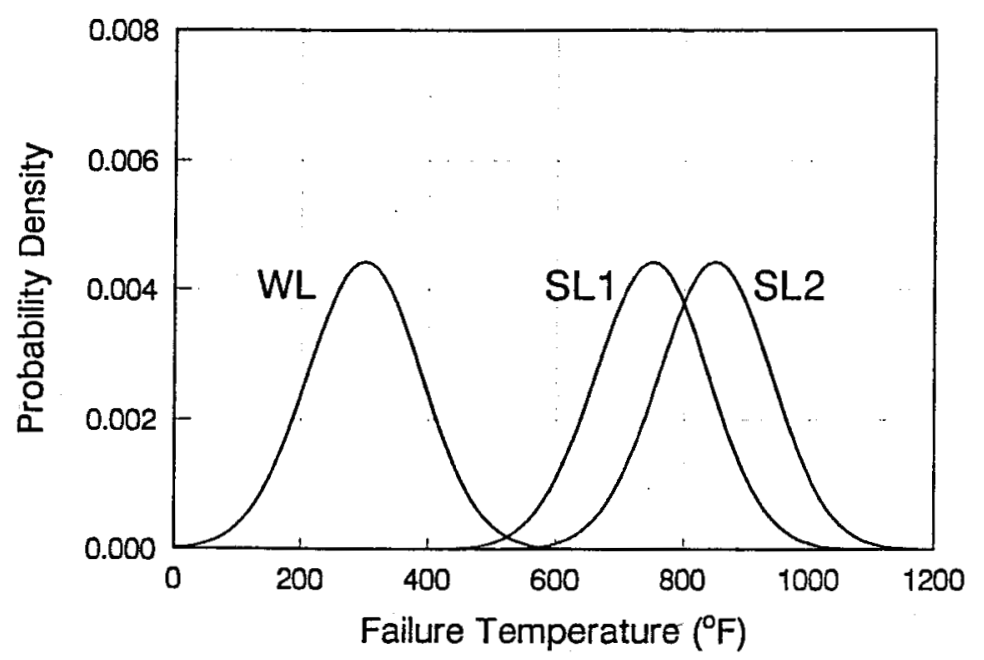

Figure 1 (b) Typical failure temperature distributions 


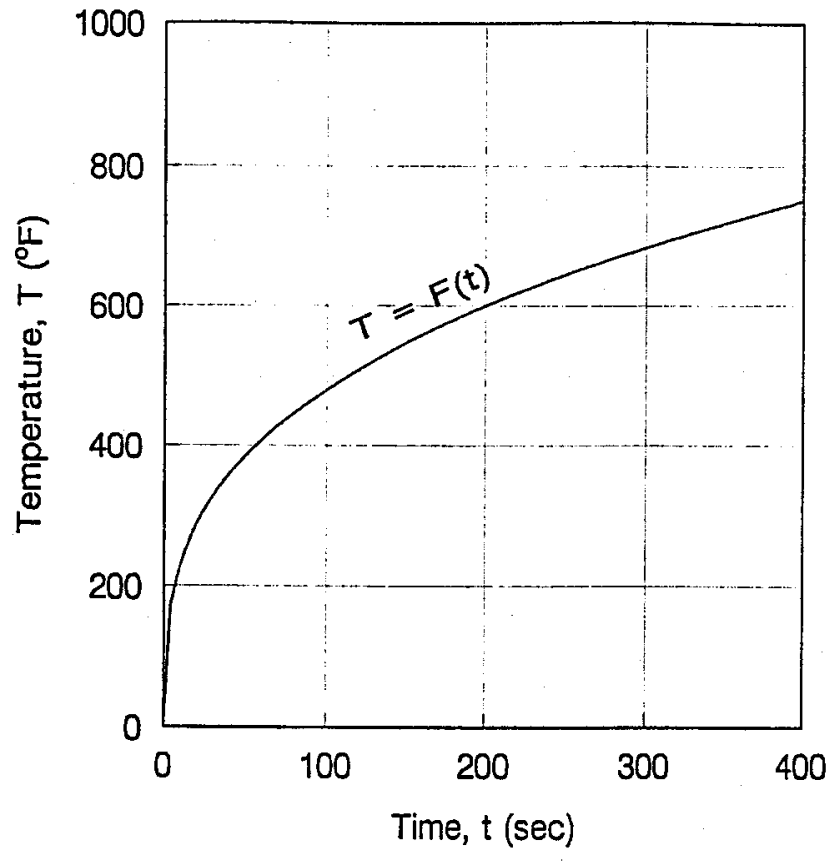

Failure

Probability$$
1.0
$$

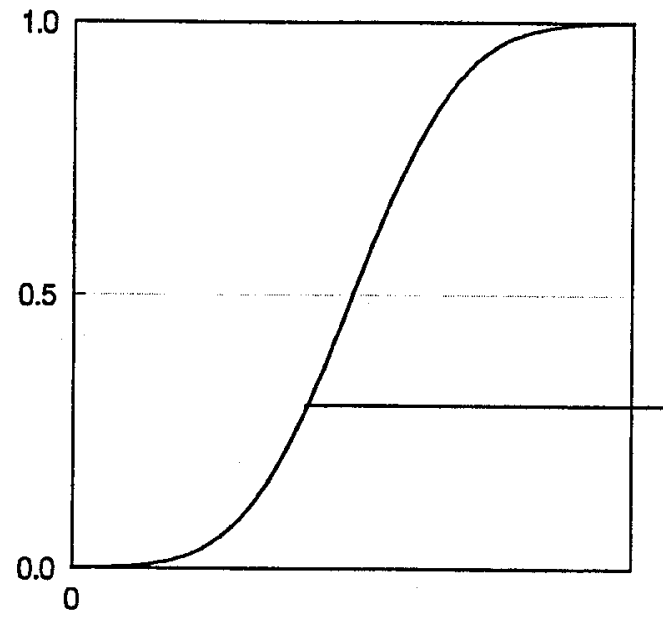

Failure Temperature, $\mathrm{T}$
Failure

Probability

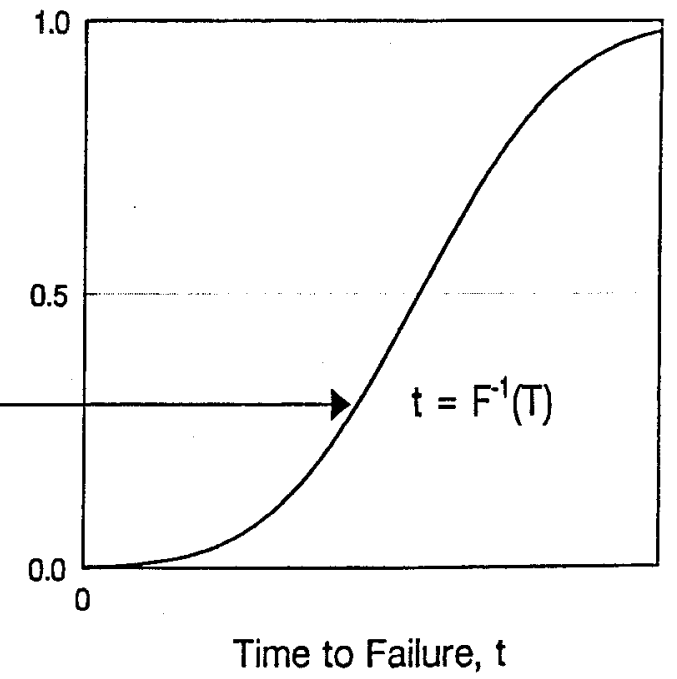

Figure 2 Derivation of time to failure distribution 
2) Separate consideration of random (irreducible) uncertainty and systematic (reducible) modeling uncertainty requires a dual (inner/outer) Monte Carlo sampling process which is computationally inefficient.

3) Sampling uncertainty is introduced, and must be considered in evaluating the precision of the quantification.

4) Consideration of interdependencies between the failures of the strong links and weak links is awkward, and requires the generation of appropriately correlated sets of sample members for each Monte Carlo realization. (This is computationally time consuming).

5) Commonly-used approximations (such as assuming linearity in generating time to failure distributions for each strong and weak link) may introduce errors in the final results.

This white paper presents an alternative integral formulation for the calculation of the probability of such strong link/weak link cutsets. This formulation allows explicit incorporation of uncertainties in both the temperature-time histories of each element as well as uncertainties in the failure temperatures for each element. Being an integral formulation, the calculation is direct, and no sampling uncertainty is introduced. It also allows direct incorporation of arbitrary correlation between the strong link failures, as well as allowing for explicit propagation of both random and systematic uncertainties throughout the calculational process. (However, the weak link failures are considered independent of the strong link failures in this formulation.) Finally, it does away with the necessity of directly evaluating the time to failure probability distributions, and instead, requires only that temperature-time histories be available for each element.

This white paper is divided into three parts. In the first part (Section 2.0), background is provided on the different types of uncertainty (random vs. systematic) and the rationale/value of keeping these uncertainties separate during the calculational process. The second part (Section 3) presents a derivation of the applicable integral formulations, first in the time domain and then in the temperature domain. Applications of the integral formulation are presented in Section 3 illustrating the effects of including uncertainty in both the calculated temperature responses as well as in the failure temperatures, and the effects of correlation between strong link failures. Use of the integral formulations to develop mean point estimates of the race cutset failure probabilities is described briefly in Section 4. Appendix A gives a Users Manual for P-RACE a FORTRAN 77 code which evaluates race cutset point estimate probabilities using the integral formulation presented in this white paper. 


\subsection{Uncertainty and Correlation Considerations.}

\subsection{Single Compenent Failure}

Consider a single component whose failure probability is specified by a single failure temperature distribution as shown in Figure 3. This model assumes that the component fails only as a function of peak temperature reached. The distribution implies that a group of nominally identical components (denoted Group 1) was thermally heated and, as the temperature was increased, the number of failures was counted. The components failed at different temperatures due to (essentially) random variations in tolerances, material properties, solder thicknesses, bubbles, etc. In effect, these small random variations are not measurable and hence not knowable. No matter how many units are tested, they will always fail over a range of temperatures.

Given a single component from this group of nominally identical components, we cannot predict with certainty at what peak temperature it will fail, but the distribution of failure temperature derived from the testing of multiple components allows us to estimate the probability that it will fail as a function of peak temperature.

Consider now another group of nominally identical components (Group 2) which are nominally identical to the first group (i.e., built to the same specifications). But, assume the second group was built by a different manufacturer, or was built using a different heat (batch) of material (glue, solder, etc.) such that, when this second group is tested as before, a (statistically significant) different distribution is obtained as shown in Figure 4. We could, if desired, test samples of glue, solder, etc. to understand the cause of the systematic shift in failure characteristics. If we were able to characterize the systematic shift, then the only uncertainty remaining would be that due to randomness (present for both groups.) However, in performing an evaluation of the probability of failure of a given component, we usually do not have the luxury of such experimentation, and usually have (at best) failure data on only a single batch of components. But it is still important to reflect both the randomness and the systematic uncertainty in our assessment, as the latter provides confidence bounds on our results. Often, limited data in conjunction with expert judgment (based on unquantified but applicable experience) is used to estimate the systematic uncertainty.

Mathematically, this can be done by characterizing the failure probability of the (nominally identical) components by a family of failure probability distributions. This can be a discrete family of curves, or a family of known distributional form with prescribed uncertainty on the defining parameters of the distribution. For example, an often-used family of distributions is the log-normal family defined over $(0, \infty)$ as shown in Figure 5 . Each member of the family is characterized by a median and a random uncertainty $\beta_{\mathrm{r}}$ (i.e., the standard deviation of the logarithms of the random variate.) The systematic uncertainty is characterized by uncertainty on the median parameter, as specified by $\beta_{u}$. This simple form is useful when little is known about the systematic component of total uncertainty. 


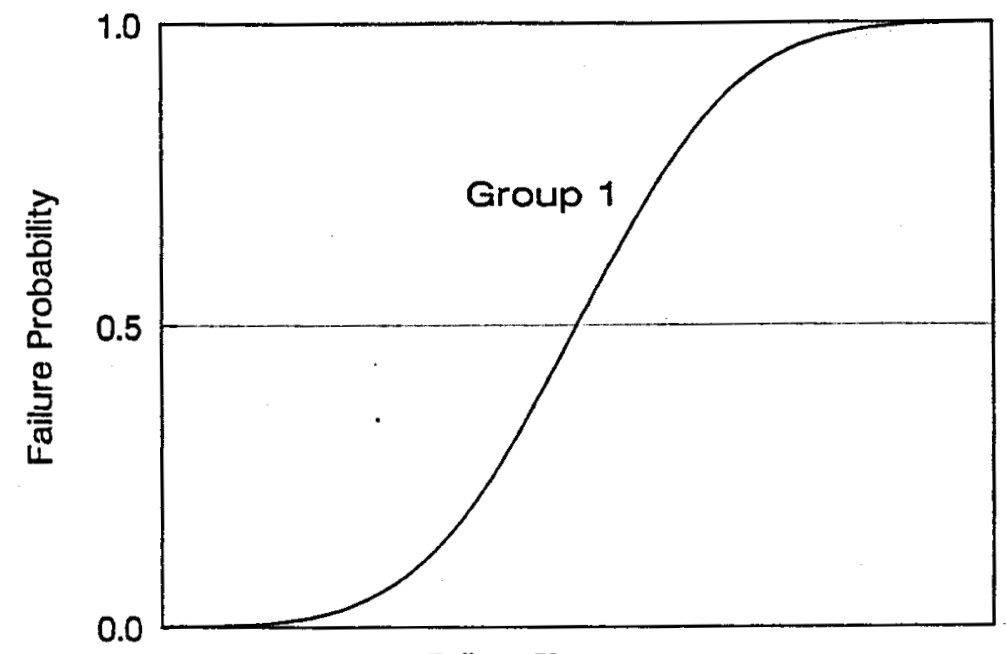

Failure Temperature

Figure 3 Example of failure temperature cumulative distribution function

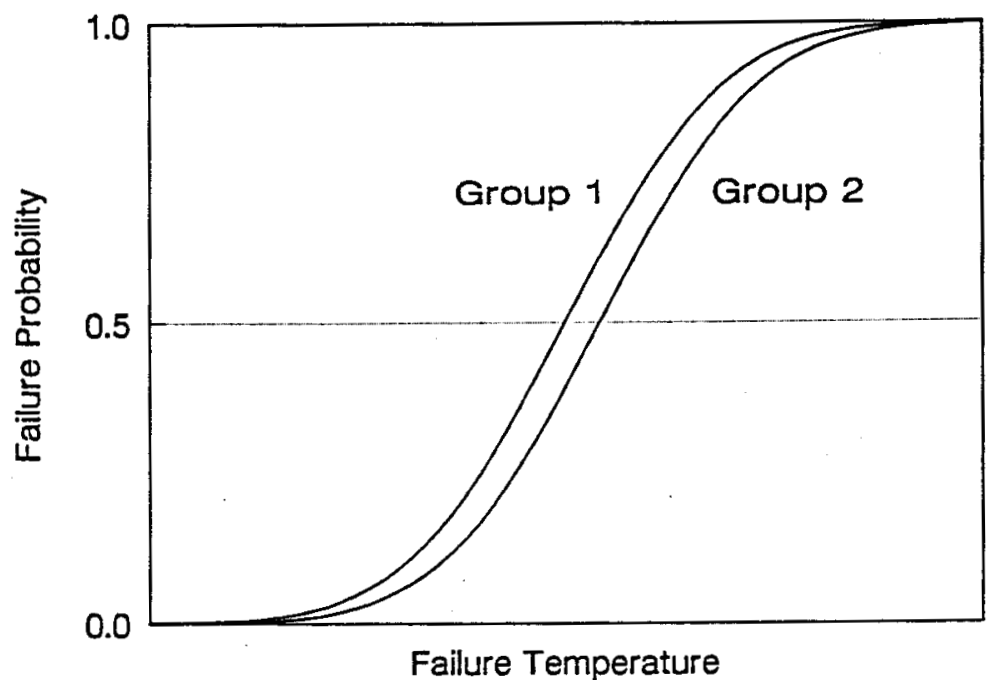

Figure 4 Example of multiple failure temperature distribution functions 


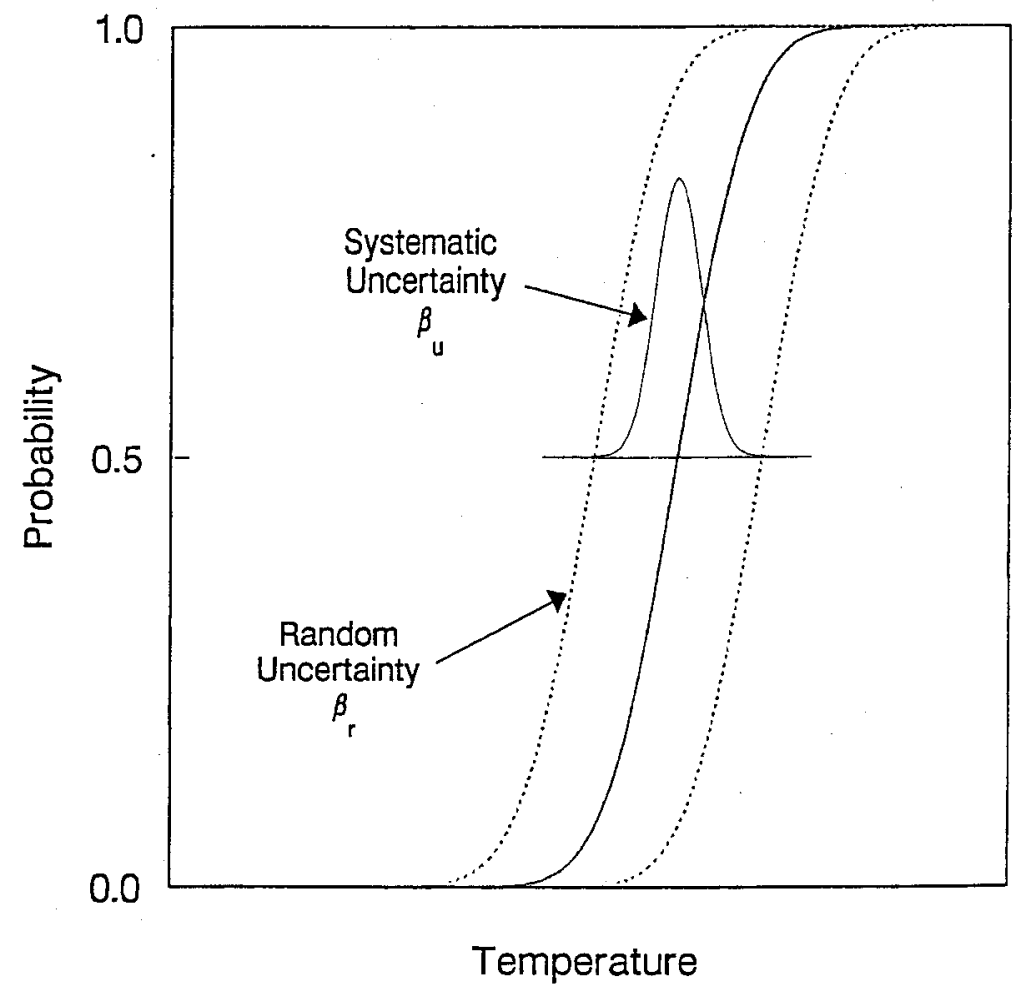

Figure 5 Typical log normal family of failure temperature curves 
If the peak temperature reached by a component is known exactly, and if there is no systematic uncertainty, then the probability of failure is read directly off the (single) failure temperature distribution. It is, of course, a single (point estimate) value, say 0.3 as shown in Figure 6.

If the peak temperature reached by a component is known exactly, but there is significant systematic uncertainty, then as described above, the failure temperature is characterized by a family of distributions [as in Figure 7(a)] and there is uncertainty in the predicted failure probability as shown in Figure 7 (b). Now we can make statements such as:

(1) Taking into account both random and systematic uncertainties, the mean (expected value) of the probability of failure of a component heated to $\mathrm{T}_{\max }$ is 0.3 .

(2) With $90 \%$ confidence the probability of failure of this component heated to a temperature $\mathrm{T}_{\max }$ is between 0.2 and 0.4 .

(3) There is some specific temperature $T_{\max }$ for which we are $95 \%$ confident that less than $5 \%$ of components tested to $T_{\max }$ will fail.

Further, we can determine how much the confidence bounds on the failure probability would decrease if we perform additional testing to reduce the systematic component of uncertainty.

Consider now the case where the peak temperature reached by the component is not known exactly. (The peak temperature could be either measured or predicted by a computer code.) It may also have both random and systematic components of uncertainty. The randomness is due to random variations in gap sizes, tolerances, surface roughnesses, etc. If a computer code was used to predict the peak temperature, then multiple computer runs would have to be made (while randomly varying the model parameters) to generate the randomness in the predicted temperatures. There may also be systematic uncertainty due to the model, method of solution, approximate equations in the code, etc., which -- in principle -- could be reduced or eliminated by use of more accurate models, better calibration to data, etc.

Thus, the peak temperature may also be characterized by a family of distributions to reflect both random and systematic uncertainty. In this case, we again generate a distribution on the probability of failure given the uncertainty distributions on $\mathrm{T}_{\max }$ and on $\mathrm{T}_{\text {fail }}$. In general, the failure probability distribution can be constructed by randomly sampling the systematic uncertainty parameters of the temperature response and failure temperature distributions so as to obtain a sample realization of one $T_{\max }$ distribution and one $T_{\text {fail }}$ distribution, and then evaluating the (single) failure probability value obtained by convolving these two sample distributions using the well-known stress-strength interference equation:

$$
P_{\text {fail }}=\int F_{\text {fail }}(T) f_{T}(T) d T
$$




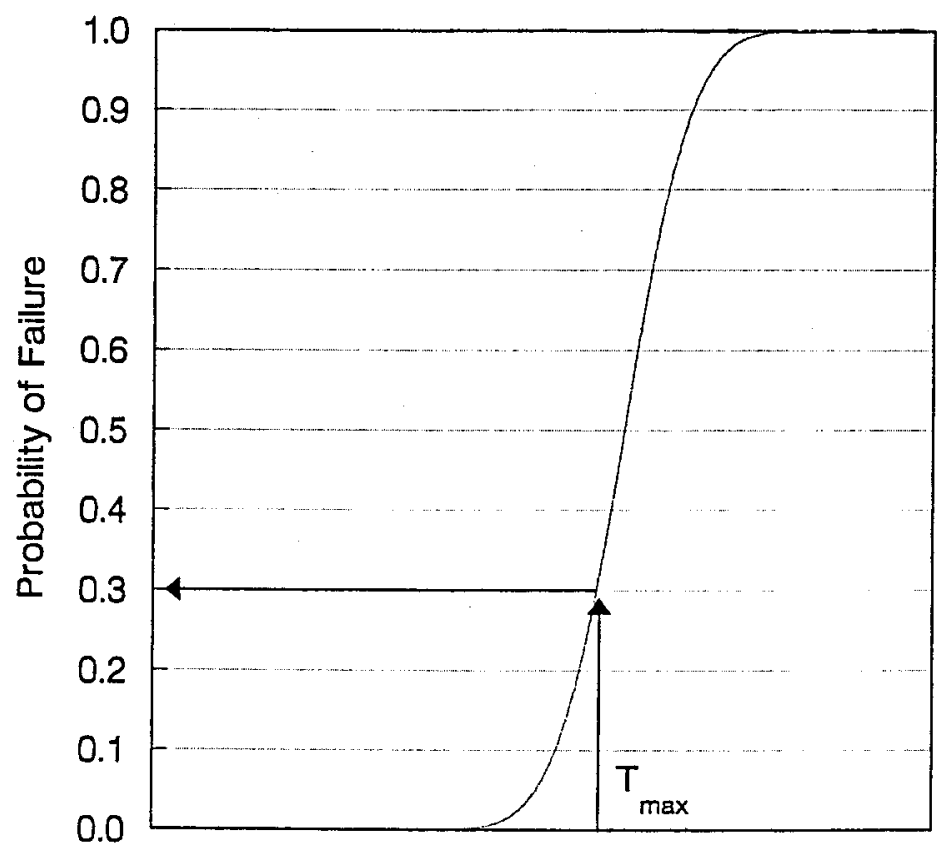

Failure Temperature, $\mathrm{T}$

Figure 6 Point estimate failure probability calculation 


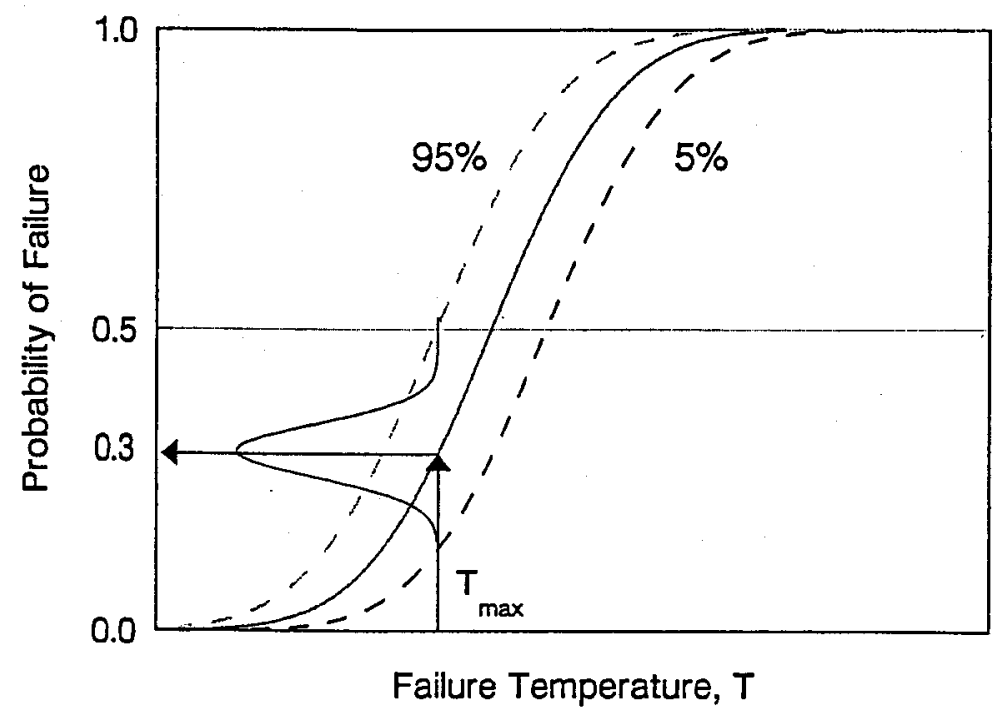

Figure 7 (a) Uncertainty in failure probability calculation given family of curves

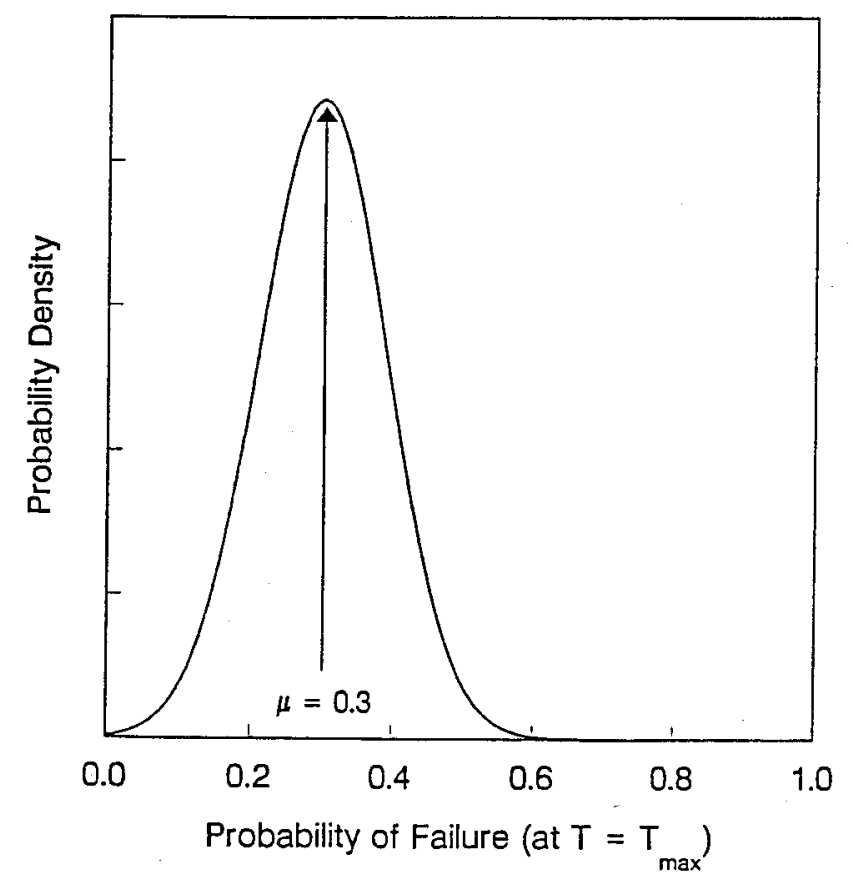

Figure 7 (b) Uncertainty distribution on failure probability 
where $F_{\text {fail }}(T)$ is the CDF of the distribution of failure temperature and $f_{T}(T)$ is the pdf of $T_{\max }$. After multiple (random) realizations of $P_{\text {fail }}$ are obtained, the distribution on $P_{\text {fail }}$ may be plotted, the associated statistics computed, and confidence bounds obtained.

\subsection{Multiple Component Failures}

When dealing with the simultaneous failure of multiple components, the possibility of correlation (from several sources) must be considered. For example, if a computer code is used to predict the temperature-time responses, and if multiple computer runs are made (systematically varying the random model parameters) so as to characterize the random uncertainty in the output temperature-time histories of the strong and weak links, then it is likely that the computed temperature responses are highly correlated. That is, if (due to a specific set of random variations in input parameters) one strong link temperature-time history is higher than average, then the temperature-time history of another strong link is also somewhat higher than average. The fact that this pair of temperature-time histories "shifts together" can have a profound impact on the quantification of a cutset involving the simultaneous failure of these two strong links. The result of neglecting this correlation is always non-conservative.

In a similar fashion, there can be correlation between the failure temperatures of two or more components. This can, in principle, only be determined by pair-wise testing of multiple components. However, when the randomness in failure temperature is due to small random variations in gaps, dimensions, etc., the correlation between the failure temperatures of two components would be expected to be small. However, if the failure modes of the two components are due to failure of a common subcomponent (say, melting of a similar type of electrical insulation) present in both components, then the failure temperatures of the two components would be expected to be highly correlated.

Given that we know (or have estimated) correlations between the temperature-time histories of the multiple components and between their failure temperatures, we can compute the joint probability of failure of the components (at any instant of time) by constructing a multivariate probability density function. This is not, in general, very easy for arbitrary distributions on the failure temperatures and computed temperature responses. However, in the case where the random uncertainties in the computed temperature histories and in the failure temperatures are either normal or log normal, explicit representations are available. For example, if normal distributions are assumed, then we can define

$$
\begin{aligned}
& Z_{1}=T_{1}-F_{1} . \\
& Z_{2}=T_{1}-F_{2} .
\end{aligned}
$$

where $T_{i}$ are the computed temperatures of the components (with associated random uncertainties characterized by $\sigma_{T, i}$ ) and $F_{i}$ are the failure temperatures for each of the components (with associated random uncertainties characterized by $\sigma_{F, i}$.) Since $T_{i}$ and $F_{i}$ are assumed to be 
normal random variables, then the $Z_{\mathrm{i}}$ are also normal random variables with known means and standard deviations. In this case, the probability of the joint failure of the components at any instant of time can be expressed as

$$
P\left[Z_{1}>0, Z_{2}>0, \ldots, Z_{n}>0\right]=\int \ldots \iint f_{Z}\left(Z_{1}, Z_{2}, \ldots, Z_{n}\right) d Z_{1} d Z_{2} \ldots . d Z_{n}
$$

where

$$
\mathrm{f}_{\mathrm{Z}}=\frac{1}{(2 \pi)^{n / 2}\left(\Sigma_{z}\right)^{1 / 2}} \exp \left\{0.5\left(\overrightarrow{\mathrm{Z}}-\vec{\mu}_{z}\right)^{T}\left(\Sigma_{z}\right)^{-1}\left(\overrightarrow{\mathrm{Z}}-\vec{\mu}_{z}\right)\right\}
$$

In this expression, $\vec{Z}$ and $\vec{\mu}_{z}$ are vectors given by

$$
\begin{aligned}
& \vec{Z}=\left(Z_{1}, Z_{2}, \ldots ., Z_{n}\right) \\
& \vec{\mu}_{z}=\left(\mu_{1}, \mu_{2}, \ldots ., \mu_{n}\right)
\end{aligned}
$$

and $\Sigma_{\mathrm{z}}$ is the correlation matrix whose elements are given by

$$
\Sigma_{i j}=\operatorname{Cov}\left(T_{i}, T_{j}\right)+\operatorname{Cov}\left(F_{i}, F_{j}\right)-\operatorname{Cov}\left(T_{i}, F_{j}\right)-\operatorname{Cov}\left(T_{j}, F_{i}\right)
$$

and $\mathrm{COV}$ denotes the covariance between two random variables, i.e.,

$$
\operatorname{COV}(\mathrm{x}, \mathrm{y})=\mathrm{E}\{[\mathrm{x}-\mathrm{E}(\mathrm{x})][\mathrm{y}-\mathrm{E}(\mathrm{y})]\}
$$

The standardized measure of correlation between two random variables is the coefficient of correlation defined by

$$
\rho_{x, y}=\frac{\operatorname{COV}(x, y)}{\sigma_{x x} \sigma_{y y}}
$$

where $\sigma_{\mathrm{xx}}, \sigma_{\mathrm{yy}}$ are the corresponding standard deviations of $\mathrm{x}$ and $\mathrm{y}$. The correlation coefficient between any two random variables can be estimated from pairwise sample data by well-known equations analogous to the equations used for estimating sample standard deviations.

In general, the evaluation of the multivariate joint probability of simultaneous failure of a group of components using Equations 1 and 2 is non-trivial due to the complex integrand and the need to evaluate $\mathrm{n}$ integrals for consideration of $\mathrm{n}$ failures. For the case of two components, however, standard IMSL (or other) subroutines are available.

To illustrate the importance of correlation in evaluating the strong link/weak link cutsets with multiple strong links, consider the joint failure probability of two strong links (SL1 and SL2) at a 
particular instant in time. Assume that (at this instant of time) the distributions of temperature response and failure temperature of the strong links are normal, and given by

$$
\begin{aligned}
& T_{1}=N\left(750^{\circ}, 40^{\circ}\right) \\
& T_{2}=N\left(800^{\circ}, 40^{\circ}\right) \\
& F_{1}=N\left(900^{\circ}, 30^{\circ}\right) \\
& F_{2}=N\left(900^{\circ}, 30^{\circ}\right)
\end{aligned}
$$

where $\mathrm{N}(\mu, \sigma)$ denotes a normal distribution with mean $\mu$ and standard deviation $\sigma$.

Then the $Z_{i}=T_{i}-F_{i}$ variables are also normal, with distributions given by

$$
\begin{aligned}
& Z_{1}=N\left(-150^{\circ}, 50^{\circ}\right) \\
& Z_{2}=N\left(-100^{\circ}, 50^{\circ}\right)
\end{aligned}
$$

In this case, Equation (1) reduces to

where

$$
\mathrm{P}\left[\mathrm{Z}_{1}>0, \mathrm{Z}_{2}>0\right]=\iint f_{z}\left(\mathrm{Z}_{1}, \mathrm{Z}_{2}\right) \mathrm{d} \mathrm{Z}_{1} \mathrm{~d} \mathrm{Z}_{2}
$$

$$
\begin{aligned}
f_{z}\left(Z_{1}, Z_{2}\right) & =\frac{1}{2 \pi \sigma_{1} \sigma_{2} \sqrt{1-\rho^{2}}} * \\
& * \exp \left\{\frac{-1}{2\left(1-\rho^{2}\right)}\left[\left(\frac{Z_{1}-\mu_{1}}{\sigma_{1}}\right)^{2}-\frac{2 \rho\left(Z_{1}-\mu_{1}\right)\left(Z_{2}-\mu_{2}\right)}{\sigma_{1} \sigma_{2}}+\left(\frac{Z_{2}-\mu_{2}}{\sigma_{2}}\right)^{2}\right]\right\}
\end{aligned}
$$

or, for simplicity,

$$
\mathrm{P}\left[Z_{1}>0, Z_{2}>0\right]=1-\mathrm{B}\left[\mu_{1}, \mu_{2}, \sigma_{1}, \sigma_{2}, \rho\right]
$$

where $B[]$ in the standard Binormal probability distribution. Figure 8 shows the probability of simultaneous failure of both strong links (at this instant of time) as a function of the correlation coefficient.

For $\rho=0$, the two failure events are independent, and the joint failure probability is

$$
\begin{aligned}
\mathrm{P}\left[\mathrm{Z}_{1}>0, \mathrm{Z}_{2}>0\right] & =\mathrm{P}\left[\mathrm{Z}_{1}>0\right] * \mathrm{P}\left[\mathrm{Z}_{2}>0\right] \\
& =(1.35 \mathrm{e}-3)(2.28 \mathrm{e}-2) \\
& =3.07 \mathrm{e}-5
\end{aligned}
$$




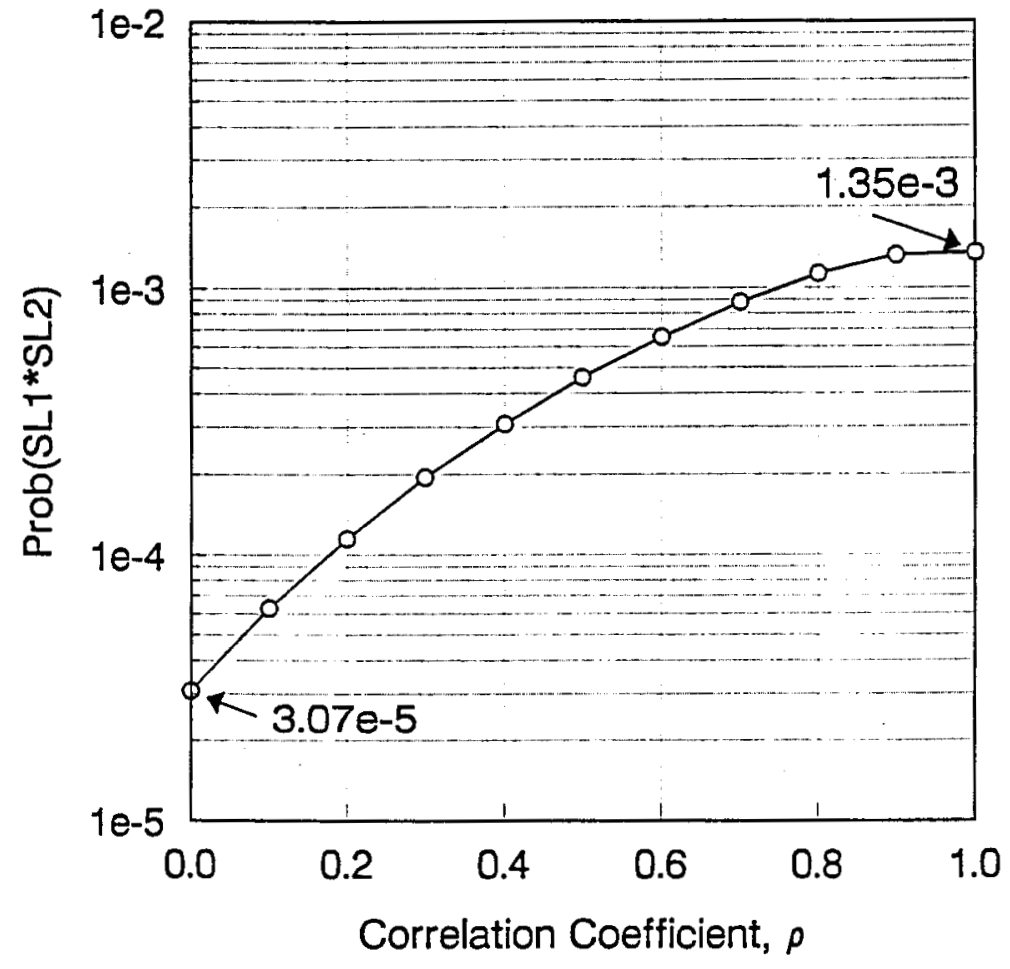

Figure 8 Probability of joint failure of two strong links as a function of correlation coefficient 
where the numerical values for the independent failure probabilities are taken directly from the known normal probability distributions for the $\mathrm{Z}_{\mathrm{i}}$, that is,

$$
\begin{aligned}
& P\left[Z_{1}>0\right]=1-\Phi\left\{\frac{0-(-150)}{50}\right\}=1.35 e-3 \\
& P\left[Z_{2}>0\right]=1-\Phi\left\{\frac{0-(-100)}{50}\right\}=2.28 e-2
\end{aligned}
$$

and $\Phi[]$ is the $\mathrm{N}(0,1)$ standard normal probability distribution.

By contrast, when the failure events are fully correlated $(\rho=1)$, then the probability of their joint failure is

$$
\begin{aligned}
\mathrm{P}\left[\mathrm{Z}_{1}>0, \mathrm{Z}_{2}>0\right] & =\operatorname{Min}\left\{\mathrm{P}\left[\mathrm{Z}_{1}>0\right], \mathrm{P}\left[\mathrm{Z}_{2}>0\right]\right\} \\
& =1.35 \mathrm{e}-3
\end{aligned}
$$

which is a factor of 45 greater than the independent failure value. In effect, when the two failure events are fully correlated, the probability of both elements failing simultaneously reduces to the probability of failure of the strongest element.

Hence it can be seen that correlation can make a very significant difference when evaluating the joint probability of two or more failure events. Thus it is, in general, non-conservative to neglect correlation between two or more strong links when computing the probability of all strong links failing before a weak link fails in a race cutset. In the following section, it will be shown how correlation between strong link failures can be included as part of the integral formulations.

\subsection{Integral Race Cutset Probability Formulations}

In the following two sections, integral formulations for computing the (point estimate) probability that all strong links fail before a weak link fails are derived. The first formulation is in the time domain, and is the direct counterpart to the Monte Carlo approach described in Section 1.0. As such, it suffers from many of the same limitations, although no sampling uncertainty is introduced. However, it conceptually leads to a formulation in the temperature domain, which is the subject of main interest in this white paper.

\subsection{Integral Formulation in the Time Domain}

With no loss in generality, we can consider two strong links (SL1 and SL2) and one weak link (WL1). We assume that, using temperature-time histories and probability distributions on the failure temperatures for each of the strong and weak links, we have previously derived (as 
discussed earlier in Section 1.0) probability distributions for the times to failure of each link, denoted $t_{\mathrm{SL} 1}, \mathrm{t}_{\mathrm{SL} 2}$ and $\mathrm{t}_{\mathrm{WL} 1}$. We wish to evaluate

$$
\mathrm{P}(\mathrm{SL} 1 * \mathrm{SL} 2 * \overline{W L 1})=\mathrm{P}\left(\mathrm{t}_{\mathrm{SL} 1}<\mathrm{t}_{\mathrm{WL} 1} \text { and } \mathrm{t}_{\mathrm{SL} 2}<\mathrm{t}_{\mathrm{WL} 1}\right)
$$

This can equivalently be expressed as

"What is the probability that WL1 has failed in a certain increment of time $\left(\mathrm{t}_{\mathrm{o}}-\Delta t / 2, t_{0}+\Delta t / 2\right)$, and that the strong links have already failed?"

Thus we ask for the joint probability of three events:

$$
\begin{aligned}
& \text { Event } \mathrm{A}=\mathrm{t}_{\mathrm{SL1}}<\mathrm{t}_{\mathrm{o}} \\
& \text { Event } \mathrm{B}=\mathrm{t}_{\mathrm{SL2}}<\mathrm{t}_{\mathrm{o}} \\
& \text { Event } \mathrm{C}=\mathrm{t}_{\mathrm{o}}-\Delta t / 2<t_{W L 1}<t_{o}+\Delta t / 2
\end{aligned}
$$

Hence in the time increment $\Delta t$,

$$
\begin{aligned}
\mathrm{P}(\mathrm{SL} 1 * \mathrm{SL} 2 * \overline{W L 1}) & =P\left(t_{S L 1}<t_{o}\right) * P\left(t_{S L 2}<t_{o}\right) * P\left(t_{o}-\Delta \mathrm{t} / 2<\mathrm{t}_{\mathrm{WL} 1}<t_{o}+\Delta t / 2\right) \\
& =C D F_{S L 1}\left(t_{o}\right) * C D F_{S L 2}\left(t_{o}\right) * P D F_{W L 1}\left(t_{o}\right) \Delta t
\end{aligned}
$$

where

$$
\begin{aligned}
& \mathrm{CDF}_{\mathrm{SL1}}=\text { Cumulative probability distribution function for } \mathrm{t}_{\mathrm{SL1}} \\
& \mathrm{CDF}_{\mathrm{SL} 2}=\text { Cumulative probability distribution function for } \mathrm{t}_{\mathrm{SL2}} \\
& \mathrm{PDF}_{\mathrm{WL} 1}=\text { Probability density function for } \mathrm{t}_{\mathrm{WL1}}
\end{aligned}
$$

Note that both events A and B are dependent on Event $C$ (through the time $t_{0}$ ). In multiplying these three probabilities together, we are applying the chain rule for conditional probabilities,

$$
\mathrm{P}\left(\mathrm{A}^{*} \mathrm{~B}^{*} \mathrm{C}\right)=\mathrm{P}(\mathrm{A} / \mathrm{BC}) * \mathrm{P}(\mathrm{B} / \mathrm{C}) * \mathrm{P}(\mathrm{C})
$$

except that, in this case, we are (initially) assuming that $\mathrm{t}_{\mathrm{SL} 1}$ and $\mathrm{t}_{\mathrm{SL} 2}$ are independent, so that

$$
\mathrm{P}(\mathrm{A} / \mathrm{BC})=\mathrm{P}(\mathrm{A} / \mathrm{C})
$$

Finally, we note that failure of WL1 in any given time increment $\Delta t$ implies that WL1 does not fail in any other time increment $\Delta \mathrm{t}$. Hence, these joint failures are mutually exclusive and thus we can sum them to obtain the probability that $\mathrm{t}_{\mathrm{SL} 1}<\mathrm{t}_{\mathrm{WL} 1}$ and $\mathrm{t}_{\mathrm{SL} 2}<\mathrm{t}_{\mathrm{WL} 1}$ in at least one time increment $\Delta \mathrm{t}$. Thus we have 


$$
\mathrm{P}(\mathrm{SL} 1 * \mathrm{SL} 2 * \overline{W L 1})=\int_{0}^{\infty} C D F_{S L 1}\left(t_{o}\right) C D F_{S L 2}\left(t_{o}\right) P D F_{W L 1}\left(t_{o}\right) d t_{o}
$$

which is the desired integral formulation in the time domain.

To illustrate this in application, consider the uniform distributions of failure times (for two strong links and one weak link) given by

$$
\begin{aligned}
& t_{\mathrm{SL} 1}=U[16,30] \\
& t_{\mathrm{SL} 2}=U[14,27] \\
& t_{\mathrm{WL} 1}=U[8,20]
\end{aligned}
$$

where $U[a, b]$ denotes a uniform distribution between the limits $a$ and $b$. These distributions are shown in Figure 9 (a). The corresponding cumulative distribution functions for the two strong links [as required for the integrand of Equation (4)] are shown in Figure 9 (b). Using Equation (4),

$$
\begin{aligned}
& \mathrm{P}(\mathrm{SL} 1 * \mathrm{SL} 2 * \overline{W L 1}) \quad=\mathrm{P}\left(\mathrm{t}_{\mathrm{SL} 1}<\mathrm{t}_{\mathrm{WL} 1,} \mathrm{t}_{\mathrm{SL} 2}<\mathrm{t}_{\mathrm{WL} 1}\right) \\
& =\int_{16}^{20}\left(\frac{t-16}{14}\right)\left(\frac{t-14}{13}\right)\left(\frac{1}{12}\right) d t \\
& =0.0171
\end{aligned}
$$

As verification, Monte Carlo sampling (with $10^{6}$ samples) gave the results $0.0170,0.0172$ and 0.0173 for three different initial seeds in the random sampling process.

Note that Equation 4 can be used with any distributional forms for $t_{\mathrm{SL} 1}, t_{\mathrm{SL} 2}$ and $t_{\mathrm{WL} 1}$, and that different distributions can be used for each. In general, in course, numerical integration would be used to evaluate the integral. However, if only one strong link is of interest, and if the distributions are of one of a few well-known types, then the integral can be obtained from the corresponding tabulated distributions. For example, assume $t_{\mathrm{SL} 1}$ and $\mathrm{t}_{\mathrm{WL} 1}$ are both normal distributions,

$$
\begin{aligned}
& \mathrm{t}_{\mathrm{SL1}}=\mathrm{N}(23 \mathrm{sec}, 2.333 \mathrm{sec}) \\
& \mathrm{t}_{\mathrm{WL1}}=\mathrm{N}(14 \mathrm{sec}, 2.0 \mathrm{sec})
\end{aligned}
$$

[Note that these are normal distributions approximating the uniform distributions of SL1 and WL1 in Figure 9 (a), where the mean is taken as the midpoint of the uniform distributions and the two end points are assumed to be $+/-6 \sigma$ points on the normal distributions.] Then, since the difference of two normally distributed random variables is also normally distributed,

$$
P\left(t_{S L 1}<t_{W L 1}\right)=P\left(t_{S L 1}-t_{W L 1}<0\right)
$$



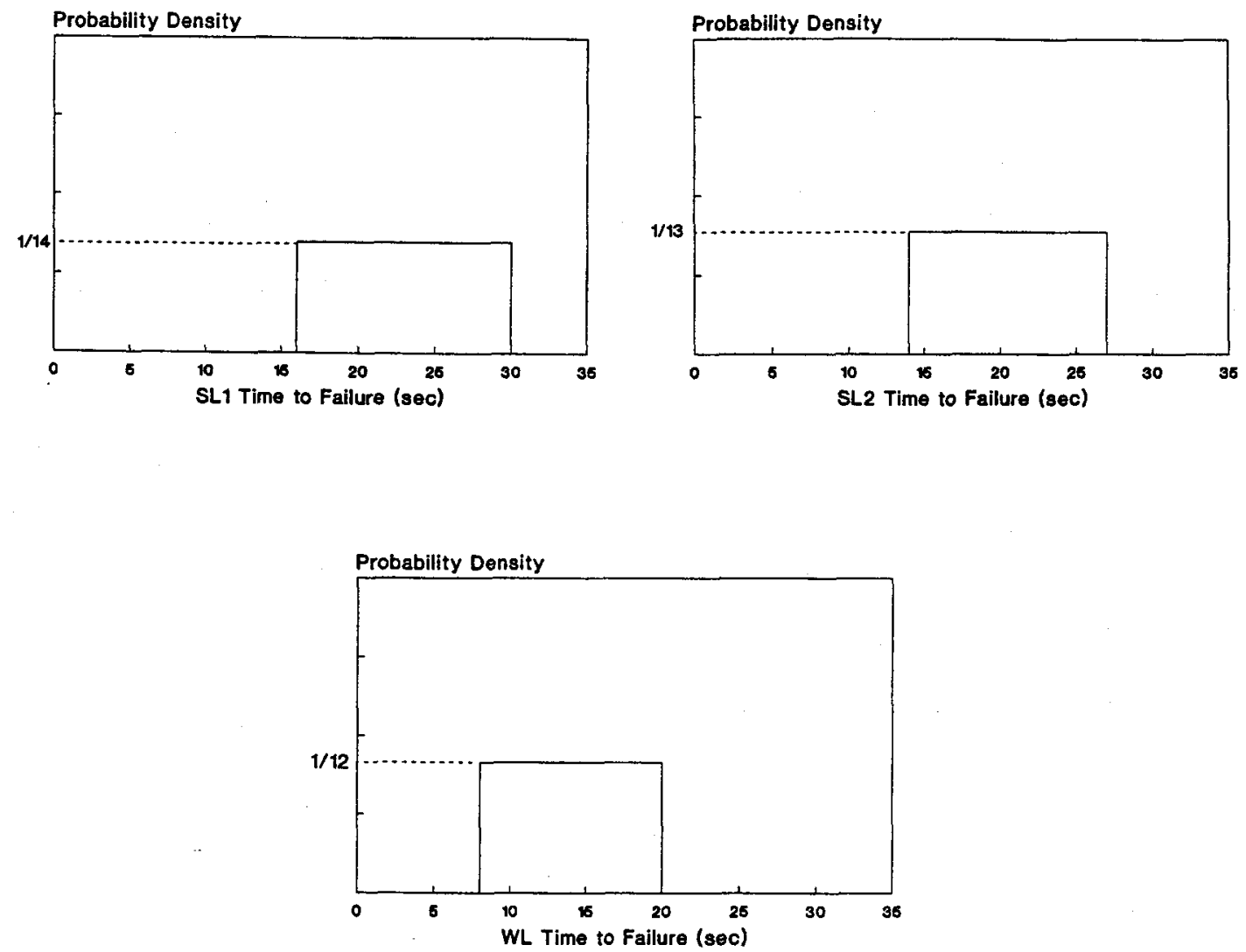

Figure 9 (a) Time to failure density functions
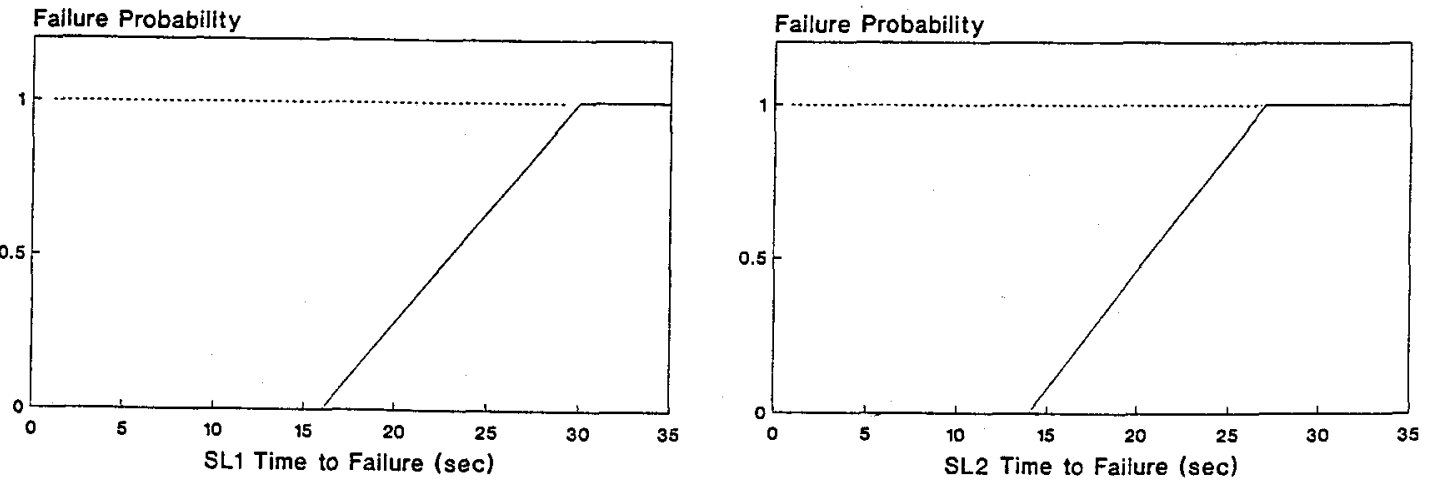

Figure 9 (b) Time to failure cumulative distribution functions 


$$
\begin{aligned}
& =\Phi\left[\frac{0-(23-14)}{\sqrt{2.333^{2}+2.0^{2}}}\right] \\
& =1-\Phi[2.93] \\
& =1.73 \mathrm{e}-3
\end{aligned}
$$

where again $\Phi[]$ is the $N[0,1]$ standard Normal probability distribution function. This probability is, of course, significantly smaller than derived from the corresponding uniform distribution example, since the uncertainty in failure times is significantly smaller.

\subsubsection{Extensions}

Clearly, the incorporation of additional strong links in the formulation is straightforward. Assuming that the strong link failure times are independent, one has

$$
\mathrm{P}\left(\mathrm{SL} 1 * \operatorname{SL} 2 *--\mathrm{-O}^{*} \mathrm{SLn}^{*} \overline{\overline{W L 1}}\right)=\int \mathrm{CDF}_{\mathrm{SL} 1}\left(\mathrm{t}_{\mathrm{o}}\right) \text {---- } \mathrm{CDF}_{\mathrm{SLn}}\left(\mathrm{t}_{\mathrm{o}}\right) \mathrm{PDF}_{\mathrm{WL} 1}\left(\mathrm{t}_{\mathrm{o}}\right) \mathrm{dt}_{\mathrm{o}}
$$

If one wishes to consider correlation between the strong link failure times, one would have to construct a multivariate cumulative probability function for the $\mathrm{n}$ strong links, and Equation (5) becomes

$$
\mathrm{P}(\mathrm{SL} 1 * \mathrm{SL} 2 * \ldots * \operatorname{SLn} * \mathrm{WL} 1)=\int \mathrm{CDF}\left(\mathrm{t}_{\mathrm{SLI}}<\mathrm{t}_{0}, \ldots, \mathrm{t}_{\mathrm{SLn}}<\mathrm{t}_{\mathrm{o}}\right) \mathrm{PDF}_{\mathrm{WL} 1}\left(\mathrm{t}_{\mathrm{o}}\right) \mathrm{dt}_{\mathrm{o}}
$$

However, construction of such a multivariate $\mathrm{CDF}$ in the time domain is non-trivial. A more straightforward way of doing this in the temperature domain is described in the next section.

\subsection{Integral Formulation in the Temperature Domain}

As before, we can consider (with no loss of generality) two strong links (SL1 and SL2) and one weak link (WL1). We also assume that we know the temperature-time histories for each of these links:

$$
\mathrm{T}_{\mathrm{SL1}}(\mathrm{t}) \quad \mathrm{T}_{\mathrm{SL} 2}(\mathrm{t}) \quad \mathrm{T}_{\mathrm{WL} 1}(\mathrm{t})
$$

Again, we pose the problem in terms of the question:

"What is the probability that WL1 fails when it experiences a temperature in the increment $\left(\mathrm{T}_{0}-\Delta T / 2, \mathrm{~T}_{0}+\Delta \mathrm{T} / 2\right)$, and that the strong links SL1 and SL2 have already failed?" 
This probability is given by

$$
\mathrm{P}_{\mathrm{SLI}}\left[\mathrm{T}_{\mathrm{SLI}}\left(\mathrm{t}_{\mathrm{o}}\right)\right]^{*} \mathrm{P}_{\mathrm{SL2}}\left[\mathrm{T}_{\mathrm{SL2}}\left(\mathrm{t}_{\mathrm{o}}\right)\right]^{*} \mathrm{f}_{\mathrm{WLI}}\left[\mathrm{T}_{\mathrm{WL} 1}\left(\mathrm{t}_{0}\right)\right] \Delta \mathrm{T}_{\mathrm{WL1}}\left(\mathrm{t}_{\mathrm{o}}\right)
$$

where, again, we initially assume independence between the strong link failures. In this expression,

$$
\begin{gathered}
P_{\mathrm{SL} 1}\left[\mathrm{~T}_{\mathrm{SL1}}\right]=\text { Probability that SL1 has failed at or below temperature } \mathrm{T}_{\mathrm{SL} 1}\left(\mathrm{t}_{\mathrm{o}}\right) \\
\mathrm{P}_{\mathrm{SL} 2}\left[\mathrm{~T}_{\mathrm{SL2}}\right]=\text { Probability that SL2 has failed at or below temperature } \mathrm{T}_{\mathrm{SL} 2}\left(\mathrm{t}_{\mathrm{o}}\right) . \\
\mathrm{f}_{\mathrm{WL} 1}\left[\mathrm{~T}_{\mathrm{WL1}}\right] \Delta \mathrm{T}_{\mathrm{WL1}}=\text { Probability that WL1 fails in the temperature increment } \\
\quad\left\{\mathrm{T}_{\mathrm{WL1}}\left(\mathrm{t}_{\mathrm{o}}\right)-\frac{\Delta \mathrm{T}_{W L 1}}{2}, \mathrm{~T}_{\mathrm{WL} 1}\left(\mathrm{t}_{0}\right)+\frac{\Delta \mathrm{T}_{W L 1}}{2}\right\}
\end{gathered}
$$

Note that $\mathrm{P}_{\mathrm{SL} 1}()$ and $\mathrm{P}_{\mathrm{SL} 2}()$ are cumulative probability distribution functions, and $\mathrm{f}_{\mathrm{WLI}}()$ is a probability density function, and all three are conditional on time.

Multiplying and dividing by $\Delta t$, and again recognizing the mutual exclusivity of failures in different time increments, we obtain

$$
\begin{aligned}
\mathrm{P}[\mathrm{SL} 1 * \mathrm{SL} 2 * \overline{W L 1}] & =\mathrm{P}\left(\mathrm{t}_{\mathrm{SL1}}<\mathrm{t}_{\mathrm{WL} 1} \text { and } \mathrm{t}_{\mathrm{SL} 2}<\mathrm{t}_{\mathrm{WL} 1}\right) \\
& =\int_{0}^{o 0} \mathrm{P}_{\mathrm{SL1}}\left[\mathrm{T}_{\mathrm{SLI}}(\mathrm{t})\right] * \mathrm{P}_{\mathrm{SL} 2}\left[\mathrm{~T}_{\mathrm{SL} 2}^{\prime}(\mathrm{t})\right] * \mathrm{f}_{\mathrm{WL} 1}\left[\mathrm{~T}_{\mathrm{WL1}}(\mathrm{t})\right] \frac{d T_{W L 1}}{d t} \mathrm{dt}
\end{aligned}
$$

This is the desired expression for failure of the strong link-weak link race in terms of failure temperature distributions. This can be seen by writing explicit expressions for the terms in the integrand. For example,

where

$$
\begin{aligned}
\mathrm{P}_{\mathrm{SL} 1}\left[\mathrm{~T}_{\mathrm{SLI}}(\mathrm{t})\right] & =\operatorname{Prob}\left[\mathrm{T}_{\mathrm{SL} 1}(\mathrm{t})>\mathrm{T}_{\mathrm{F}, \mathrm{SL} 1}\right] \\
& =\int_{0}^{o o} \operatorname{CDF}\left(\mathrm{T}_{\mathrm{F}, \mathrm{SL} 1}\right) \operatorname{PDF}\left[\mathrm{T}_{\mathrm{SL} 1}(\mathrm{t})\right] \mathrm{dT}_{\mathrm{SL} 1}
\end{aligned}
$$

$$
\begin{aligned}
& \mathrm{T}_{F, S L 1}=\text { random failure temperature of } \mathrm{SL1} \\
& \mathrm{CDF}\left(\mathrm{T}_{\mathrm{F}, \mathrm{SL} 1}\right)=\text { cumulative probability distribution function for } \mathrm{T}_{\mathrm{F}, \mathrm{SL} 1} \\
& \mathrm{~T}_{\mathrm{SL} 1}(\mathrm{t})=\text { random temperature of } \mathrm{SL} 1 \text { at time } \mathrm{t} \\
& \mathrm{PDF}\left[\mathrm{T}_{\mathrm{SL} 1}(\mathrm{t})\right]=\text { probability density function of the } \\
& \text { (calculated) temperature of } \mathrm{SL} 1 .
\end{aligned}
$$


It can be seen that $\mathrm{P}_{\mathrm{SL} 1}[]$ is nothing more than the probability that $\mathrm{SL} 1$ has failed before time $t$, but it is expressed in terms of the well-known stress-strength interference equation which involves only the (data-based) failure temperature distribution for SL1 and the uncertainty distribution for the (computer code predicted) temperature of SL1 at time t. These two required input distributions are shown schematically in Figure 10 (a). Similar expressions can be written for $P_{\mathrm{SL} 2}\left[\mathrm{~T}_{\mathrm{SL} 2}(\mathrm{t})\right]$ as shown schematically in Figure $10(\mathrm{~b})$. The density function $\mathrm{f}_{\mathrm{WL} 1}(\mathrm{t})$ can be computed as

$$
\mathrm{f}_{\mathrm{WL1}}(\mathrm{t})=\frac{\partial}{\partial \mathrm{T}_{\mathrm{WL1}}} \int_{0}^{\infty} C D F_{W L 1}\left(T_{F, W L 1}\right) P D F\left[T_{W L 1}(t)\right] d T
$$

The same pair of input distributions is required for WL1, as shown in Figure 10 (c).

Note that these expressions are written in terms of arbitrary probability distribution functions, and, in fact, all six required input distributions may have different forms. However, when the input distributions are either normal or log normal distributions, then the integrand of Equation (6) can be rapidly and efficiently calculated using the tabulated $\mathrm{N}(0,1)$ standard normal probability distribution. For example, if all input distributions are normal, i.e.,

$$
\begin{aligned}
\mathrm{T}_{\mathrm{SL} 1} & =\mathrm{N}\left[\hat{T}_{S L 1}(\mathrm{t}), \sigma_{\mathrm{T}, \mathrm{SL} 1}\right] \\
\mathrm{T}_{\mathrm{F}, \mathrm{SL} 1} & =\mathrm{N}\left[\hat{T}_{F, S L 1}(\mathrm{t}), \sigma_{\mathrm{F}, \mathrm{SL} 1}\right] \\
\mathrm{T}_{\mathrm{SL} 2} & =\mathrm{N}\left[\hat{T}_{F, S L 2}(\mathrm{t}), \sigma_{\mathrm{T}, \mathrm{SL} 2}\right] \\
\mathrm{T}_{\mathrm{F}, \mathrm{SL} 2} & =\mathrm{N}\left[\hat{T}_{F, S L 2}(\mathrm{t}), \sigma_{\mathrm{F}, \mathrm{SL} 2}\right] \\
\mathrm{T}_{\mathrm{WL} 1} & =\mathrm{N}\left[\hat{T}_{W L 1}(\mathrm{t}), \sigma_{\mathrm{T}, \mathrm{WL} 1}\right] \\
\mathrm{T}_{\mathrm{F}, \mathrm{WL} 1} & =\mathrm{N}\left[\hat{T}_{F, \mathrm{WL} 1}(\mathrm{t}), \sigma_{\mathrm{F}, \mathrm{WL} 1}\right]
\end{aligned}
$$

then, utilizing the fact that the sum or difference of two normal random variables is also a normal random variable, we can write the four terms in the integrand of Equation (6) as

$$
\begin{aligned}
P_{S L 1}(\mathrm{t}) & =\operatorname{Prob}\left[\mathrm{T}_{\mathrm{SL} 1}>\mathrm{T}_{\mathrm{F}, \mathrm{SL} 1}\right] \\
& =\operatorname{Prob}\left[\mathrm{T}_{\mathrm{F}, \mathrm{SL} 1}-\mathrm{T}_{\mathrm{SL} 1}<0\right] \\
& =\Phi\left\{\frac{\hat{T}_{F, S L 1}-\hat{T}_{S L 1}(t)}{\sqrt{\sigma_{F, S L 1}^{2}+\sigma_{T, S L 1}^{2}}}\right\}
\end{aligned}
$$



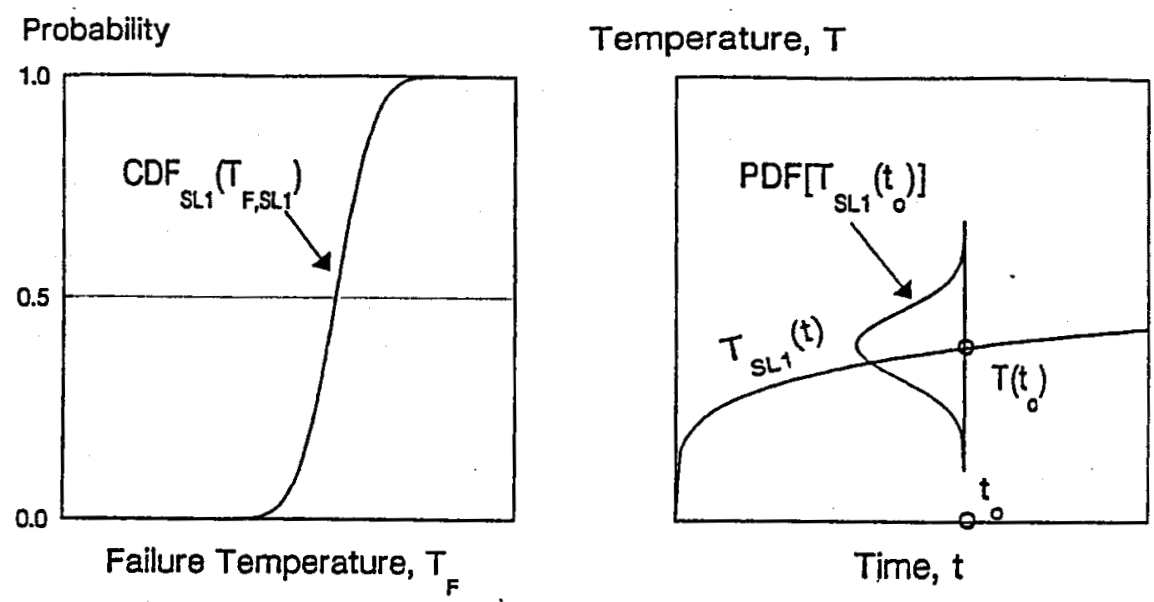

Figure 10 (a) Required input, for SL1
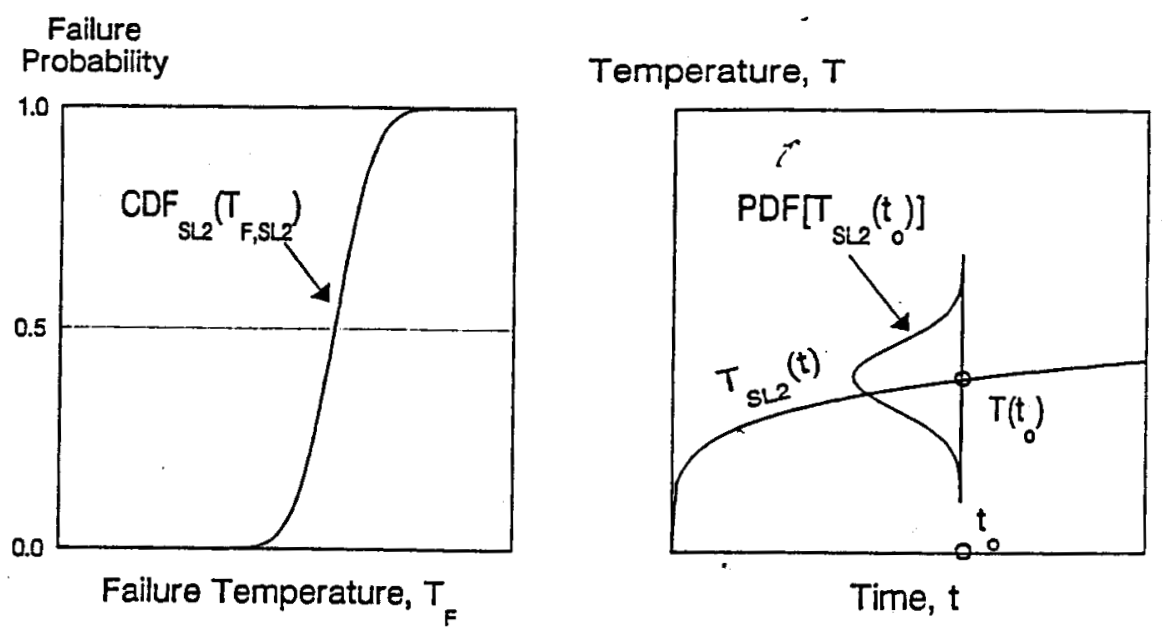

Figure 10 (b) Required input for SL2
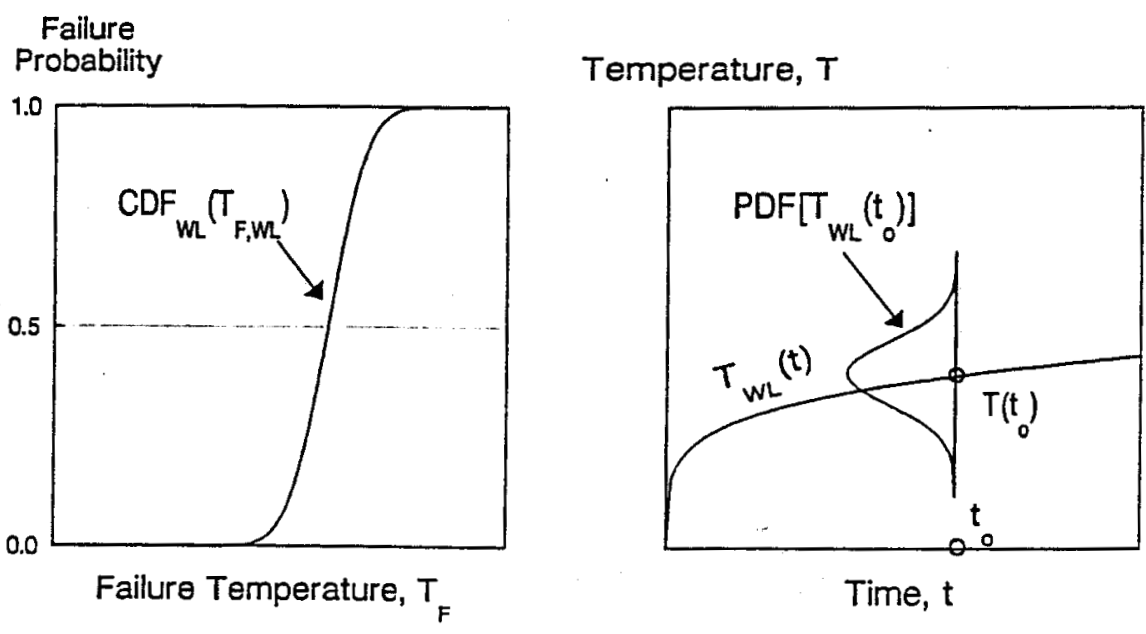

Figure 10 (c) Required input for WL1 
Similarly,

$$
\begin{aligned}
& \mathrm{P}_{\mathrm{SL2}}(\mathrm{t})=\Phi\left\{\frac{\hat{T}_{F, S L 2}-\hat{T}_{S L 2}(t)}{\left.\sqrt{\sigma_{F, S L 2}^{2}+\sigma_{T, S L 2}^{2}}\right\}}\right. \\
& \mathrm{f}_{\mathrm{WL1}}(\mathrm{t})=\frac{1}{\sigma_{W L 1} \sqrt{2 \pi}} \exp \left\{\frac{-\left[\hat{T}_{F, W L 1}-\hat{T}_{T, W L 1}\right]^{2}}{2\left[\sigma_{F, W L 1}^{2} \sigma_{T, W L 1}^{2}\right]}\right\} \\
& \frac{d T_{W L 1}}{d t}=\frac{d}{d t}\left[\hat{T}_{W L 1}(t)\right]
\end{aligned}
$$

In the above $\Phi[]$ is again the standard $N[0,1]$ normal probability distribution. Analogous equations can be written for log normal input distributions.

Numerical integration is used to evaluate the point estimate probability of a race cutset using Equation (6) with the integrand evaluated at each integration point using the above four equations. The temperature-time histories for each of the components may be input either as analytic functions or numerical data. The upper limit of integration for Equation (6) is chosen sufficiently large that the integral converges.

A computer code P-RACE was written to evaluate Equation (6) for either normal or log normal input probability distributions. As described above, input consists of the two defining parameters for each of the input distributions, and the temperature-time histories for the strong and weak links (which may be either analytic or numerical data). Up to eight strong links can be considered in any cutset. The P-RACE input and output are described in detail in Appendix A.

\subsubsection{Illustrative Examples}

(a) Example: One Strong Link and One Weak Link

Consider the case of one strong link (SL1) and one weak link (WL1). Assume that the temperature-time histories were computed to be

$$
\begin{aligned}
& \hat{\mathrm{T}}_{\mathrm{SL1}}(\mathrm{t})=60.0 \mathrm{t} \\
& \hat{\mathrm{T}}_{\mathrm{WL1}}(\mathrm{t})=70.0 \mathrm{t}
\end{aligned}
$$

and that these are median temperature-time histories. Further, assume that for any time $t$, the uncertainty distributions of the computed temperature-time histories are log normal, and characterized by log standard deviations of 


$$
\begin{aligned}
& \beta_{\mathrm{T}, \mathrm{SL} 1}=0.3 \\
& \beta_{\mathrm{T}, \mathrm{WL} 1}=0.3
\end{aligned}
$$

Note that the log standard deviation is one of the two defining parameters of a log normal distribution, and is nearly equal to the C.O.V. $=\sigma / \mu$. Thus specifying a log standard deviation of 0.3 is equivalent to saying that the standard deviation in the computed response is about 0.3 times the average.)

Further, assume that the SL1 and WL1 failure temperature distributions are also log normal, and characterized by

$$
\begin{aligned}
& \mathrm{T}_{\mathrm{F}, \mathrm{SL} 1}=\mathrm{LN}\left(900^{\circ} \mathrm{F}, \beta_{\mathrm{F}, \mathrm{SL} 1}=0.4\right) \\
& \mathrm{T}_{\mathrm{F}, \mathrm{WL} 1}=\mathrm{LN}\left(350^{\circ} \mathrm{F}, \beta_{\mathrm{F}, \mathrm{WL} 1}=0.4\right)
\end{aligned}
$$

where $\mathrm{LN}(\mathrm{m}, \beta)$ denotes a log normal distribution with median $\mathrm{m}$ and $\log$ standard deviation $\beta$. These input distributions are shown in Figure 11.

Then, inputting these parameters into P-RACE as described in Appendix A, one obtains the point estimate race cutset probability

$$
\begin{aligned}
\mathrm{P}(\mathrm{SL} 1 * \overline{W L 1}) & =\mathrm{P}\left(\mathrm{t}_{\mathrm{SL} 1}<\mathrm{t}_{\mathrm{WL} 1}\right) \\
& =0.0601
\end{aligned}
$$

This can be verified by hand calculation, due to the linearity of the temperature-time histories and the fact that the uncertainties are assumed to be log normal for both the temperature responses and the failure temperatures. To see this, note that

$$
\begin{aligned}
& \mathrm{P}\left[\mathrm{T}_{\mathrm{SL} 1}>\mathrm{T}_{\mathrm{F}, \mathrm{SL} 1}\right]=\Phi\left[\frac{\ln 60 t / 900}{0.5}\right] \\
& \mathrm{P}\left[\mathrm{T}_{\mathrm{WL}}>\mathrm{T}_{\mathrm{F}, \mathrm{WL}}\right]=\Phi\left[\frac{\ln 70 t / 350}{0.5}\right]
\end{aligned}
$$

which imply that the distributions of times to failure for these links are also both log normal, and given by

$$
\begin{aligned}
& t_{\mathrm{SLI}}=\mathrm{LN}[15 \mathrm{~s}, 0.5] \\
& \mathrm{t}_{\mathrm{WL}}=\mathrm{LN}[5 \mathrm{~s}, 0.5]
\end{aligned}
$$


Failure

Probability

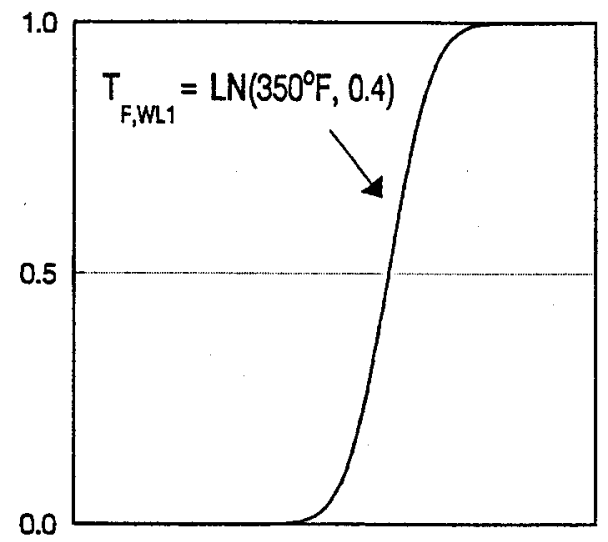

Failure Temperature, $T_{F}$
Temperature, $\mathrm{T}$

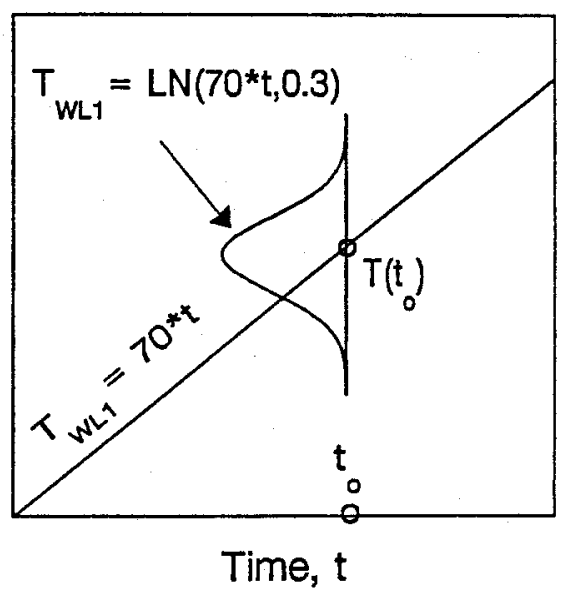

Figure 11 (a) Example Problem: Input for Weak Link
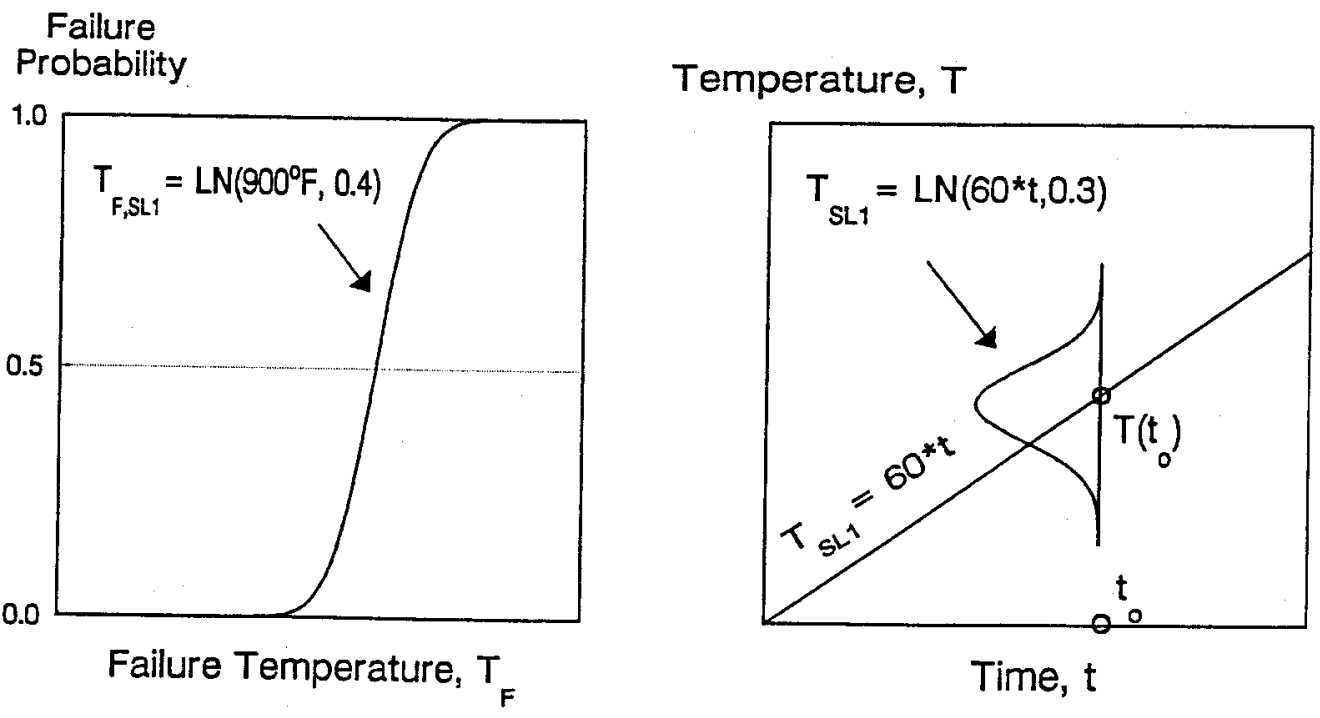

Figure 11 (b) Example Problem: Input for Strong Link 
Then

$$
\begin{aligned}
\mathrm{P}\left[\mathrm{t}_{\mathrm{F}, \mathrm{SL} 1}<\mathrm{t}_{\mathrm{F}, \mathrm{WL}}\right] & =\mathrm{P}\left[\ln \mathrm{t}_{\mathrm{SL} 1}-\ln \mathrm{t}_{\mathrm{WL}}<0\right] \\
& =\Phi\left[\frac{\ln (5 / 15)}{0.707}\right] \\
& =0.0601
\end{aligned}
$$

which is the same as computed by P-RACE using the more general formulation of Equation 6.

\section{(b) Example: Two Strong Links and One Weak Link}

Consider a race cutset with two identical strong links (seeing the same temperature time histories) and one weak link, with the strong and weak links having the same properties as in Example (a) above. Thus the input to P-RACE is

$$
\begin{aligned}
& \mathrm{T}_{\mathrm{SL} 1}=\mathrm{LN}(60.0 \mathrm{t}, 0.3) \\
& \mathrm{T}_{\mathrm{SL2}}=\mathrm{LN}(60.0 \mathrm{t}, 0.3) \\
& \mathrm{T}_{\mathrm{WL}}=\mathrm{LN}(70.0 \mathrm{t}, 0.3)
\end{aligned}
$$

for the temperature-time histories and

$$
\begin{aligned}
& \mathrm{T}_{\mathrm{F}, \mathrm{SL} 1}=\mathrm{LN}\left(900^{\circ} \mathrm{F}, 0.4\right) \\
& \mathrm{T}_{\mathrm{F}, \mathrm{SL} 2}=\mathrm{LN}\left(900^{\circ} \mathrm{F}, 0.4\right) \\
& \mathrm{T}_{\mathrm{F}, \mathrm{WL}}=\mathrm{LN}\left(350^{\circ} \mathrm{F}, 0.4\right)
\end{aligned}
$$

for the failure temperature distributions.

Assuming the strong links are independent, P-RACE gives

$$
\mathrm{P}(\mathrm{SL} 1 * \mathrm{SL} 2 * / \mathrm{WL})=0.0158
$$

This cannot be hand checked directly. However, a Monte Carlo evaluation of this (independent) case gives the results $0.0158,0.0160$, and 0.0161 for $10^{6}$ trials and three initial seeds.

Similarly, assuming that the strong links temperature responses are fully correlated and that the failure temperatures are also fully correlated, P-RACE gives

$$
\mathrm{P}(\mathrm{SL} 1 * \mathrm{SL} 2 * / \mathrm{WL})=0.0601
$$

which the same result as in Example (a) as it should be. 
The difference between the fully independent case and the fully dependent case varies with the relative capacities of the strong links and the weak link (and, of course, the prescribed uncertainties). For example, if the median failure temperature of the strong links is varied while all other parameters are held fixed, the variation of the race cutset probability (independent versus fully dependent) is as shown in Figure 12. As can be seen, the difference between the independent and dependent cases tends to increase as the capacity of the strong links increases (and hence as the race cutset probability decreases). The ratio between those two probabilities will, in general, increase as shown on this figure. For the parameters chosen, the ratio increases up to a factor of seven. Other parameters could increase the difference further.

By contrast, if one strong link capacity is held fixed at a median value of $900^{\circ} \mathrm{F}$, and the capacity of the second strong link is varied, the variation of race cutset probability is as shown in Figure 13. When the capacity of SL1 is less than SL2, the fully dependent cutset probability is dominated by (the strongest) SL2, and the cutset probability is constant. When the capacity of SL1 exceeds $900^{\circ} \mathrm{F}$, then SL1 dominates, and the cutset probabilities in both the independent and fully dependent cases decrease. The ratio between the two thus increases when the capacity of SL1 in less than SL2, and decreases when the capacity of SL1 increases past the capacity of SL2, as shown in Figure 13. Thus it can be seen that, in general, the greatest difference between the independent and fully dependent cases occurs when the two strong links have the same median capacities (and, of course, the same temperature-time histories). More generally, it can be seen that the greatest difference between the independent and fully dependent cases occurs when two (or more) strong links probability of failure versus time relations are the same. When either the capacities or the temperature-time histories vary, the net effect is to reduce the difference between the independent and fully dependent cases.

Finally, it is noted that P-RACE allows for input of arbitrary correlation between the strong link time histories (through input parameter $\rho_{r}$ ) and between the strong link failure temperatures (through input parameter $\rho_{f}$ ). Both of these input parameters can have any value between 0 and 1. Thus if actual response or capacity correlations are known, they can be input directly. Of course, for intermediate values of correlation, the cutset probability will be between the independent and fully dependent cases discussed above.

\subsubsection{Extensions}

As can be seen from the derivation, any number of additional strong links may be included by adding additional cumulative distribution functions to the integrand of Equation (6). As currently configured, P-RACE allows up to eight strong links in any one cutset.

Correlation between strong link failures can also be considered in this formulation. As before, it is only necessary to replace the independent strong link terms in the integrand of Equation (6) by the joint probability function

$$
\operatorname{CDF}\left(\mathrm{T}_{\mathrm{SL1}}<\mathrm{T}_{\mathrm{F}, \mathrm{SL} 1}, \cdots, \mathrm{T}_{\mathrm{SLn}}<\mathrm{T}_{\mathrm{F}, \mathrm{SLn}}\right)
$$




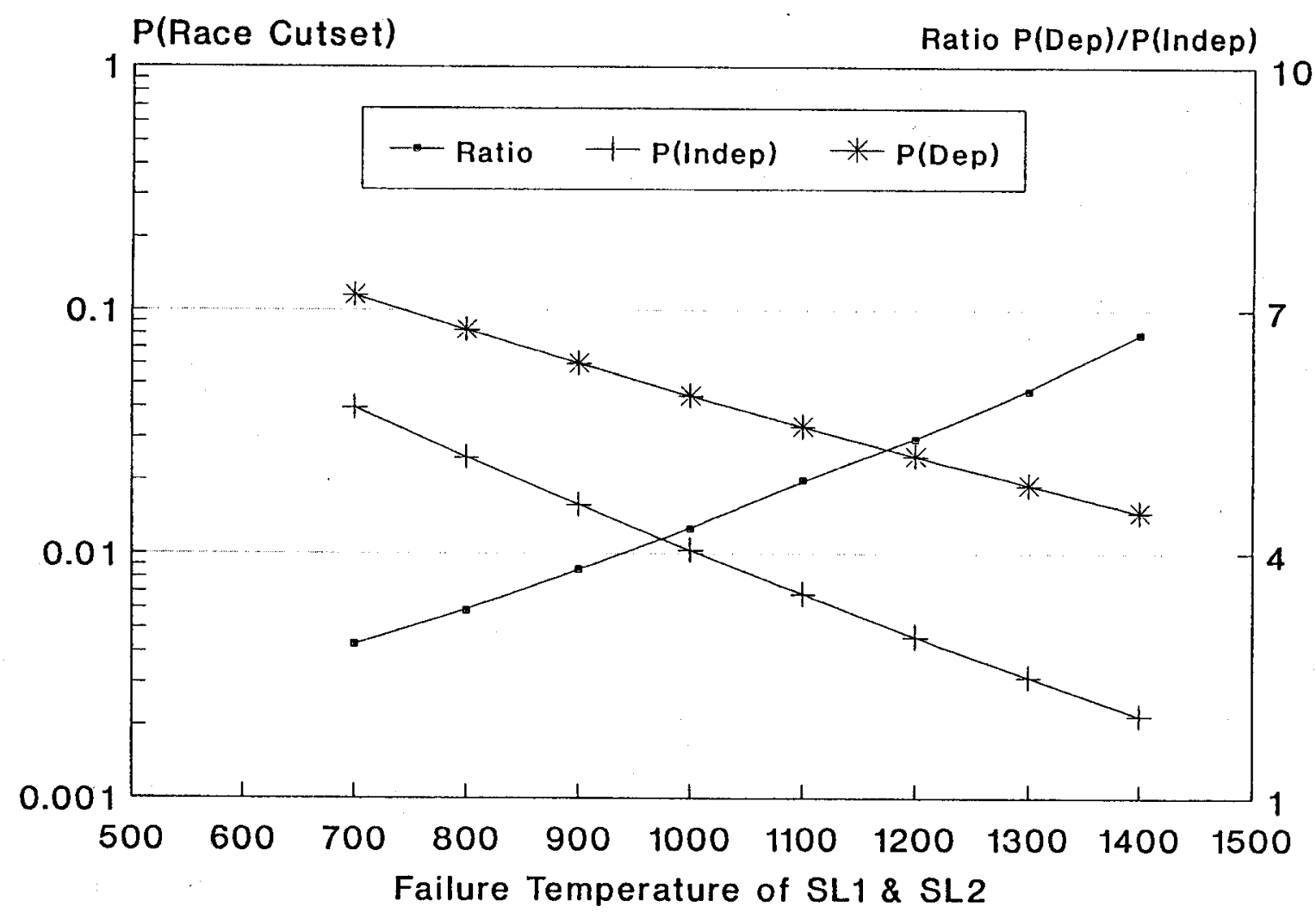

Figure 12 Effect of simultaneously varying both strong link median capacities 


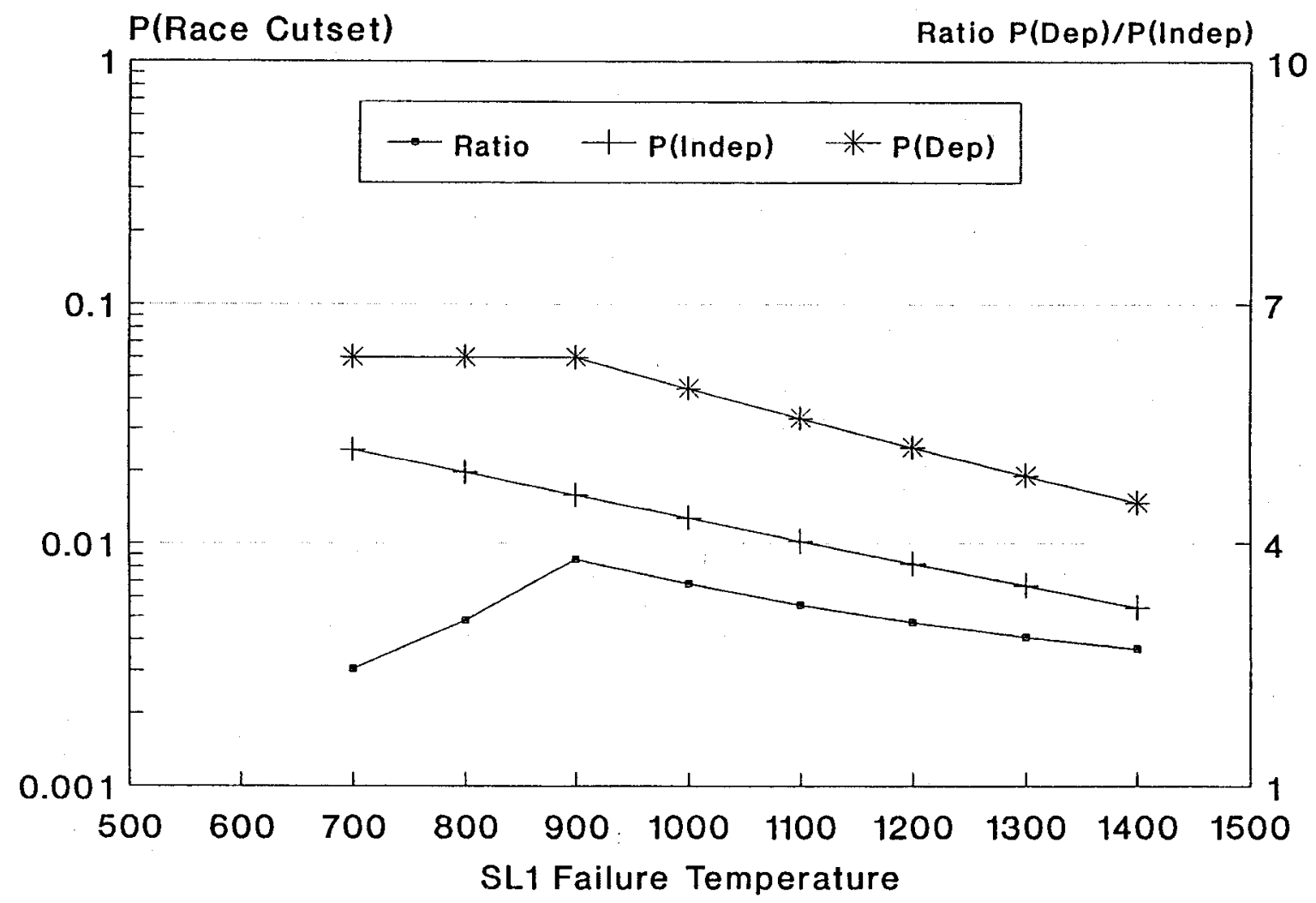

Figure 13 Effect of varying median capacity of SL1 with capacity of SL2 held fixed 
and this is exactly the same function given by Equation (1) earlier in the report. The initial version of P-RACE allows only the first two strong links to be correlated.

\subsection{Systematic Uncertainty, Race Cutset Failure Probability Distributions and Mean Point Estimates.}

As described in Section 2.0, the uncertainty in random variables can be separated into components associated with random (irreducible) uncertainty and systematic (or reducible) uncertainty, and the latter is associated with our "lack of knowledge" - which could be reduced by additional testing, analyses, etc. The two integral formulations described in this report calculate the point estimate probability of the occurrence of the race cutset considering the random (irreducible) component of uncertainty in all the input random variables. Thus, for each race cutset, a single (point estimate) value of probability is calculated by the P-RACE code.

In general, however, when both random and systematic uncertainties are included, one can calculate the distribution of the race cutset, percentiles on the distribution, and determine confidence bounds on the calculated race cutset probabilities. (For example, when the distribution of a race cutset occurrence probability is calculated, one can make such statements as "With $95 \%$ confidence, we are sure that the race cutset occurrence probability is less than 0.01 ", etc.) To compute such distributions, a Monte Carlo process can be used (at each step randomly sampling the systematic uncertainties for both the computed temperature responses and the failure temperatures and then evaluating the race cutset occurrence probability for each Monte Carlo trial). From the accumulated Monte Carlo samples of race cutset probability, the distribution and its associated statistics can be derived.

The P-RACE code described in this report does not calculate such uncertainty distributions (although work is in progress to accomplish this). However, P-RACE can be used to calculate the (point estimate) mean of the failure probability distribution - without computing the full distribution. That is, it can be shown that the true mean cutset value (for normal or log normal random failure events) can be obtained without constructing the full distribution by using the total uncertainty in evaluating the race cutset occurrence probability, where the total uncertainty is defined as

$$
\left(\sigma_{\text {total }}\right)^{2}=\left(\sigma_{r}\right)^{2}+\left(\sigma_{u}\right)^{2}
$$

and $\sigma_{\mathrm{r}}, \sigma_{\mathrm{u}}$ are the corresponding random and systematic components of uncertainty. Thus, to compute the mean point estimate, it is only necessary for the user to input total uncertainty values (in place of the random uncertainties) for each of the input distributions in the P-RACE input.

To illustrate this, consider the two strong link example in Section 3.0. The input uncertainty distributions were defined as 


$$
\begin{aligned}
& \mathrm{T}_{\mathrm{SL} 1}=\mathrm{LN}(1.0,0.3) \\
& \mathrm{T}_{\mathrm{SL} 2}=\mathrm{LN}(1.0,0.3) \\
& \mathrm{T}_{\mathrm{WL}}=\mathrm{LN}(1.0,0.3) \\
& \mathrm{T}_{\mathrm{F}, \mathrm{SL} 1}=\mathrm{LN}\left(900^{\circ} \mathrm{F}, 0.4\right) \\
& \mathrm{T}_{\mathrm{F}, \mathrm{SL} 2}=\mathrm{LN}\left(900^{\circ} \mathrm{F}, 0.4\right) \\
& \mathrm{T}_{\mathrm{F}, \mathrm{WL}}=\mathrm{LN}\left(350^{\circ} \mathrm{F}, 0.4\right)
\end{aligned}
$$

Let us assume that there is known systematic uncertainty in the median parameters of these distributions, with this uncertainty (also assumed log normal) characterized by a log standard deviation of 0.1 for both the computed temperature responses and for the failure temperatures. To compute the mean point estimate (of the true failure probability distribution), we combine the random and systematic uncertainties into the corresponding total uncertainties, and hence the input distributions became

$$
\begin{aligned}
& \mathrm{T}_{\mathrm{SL} 1}=\mathrm{LN}(1.0,0.316) \\
& \mathrm{T}_{\mathrm{SL} 2}=\mathrm{LN}(1.0,0.316) \\
& \mathrm{T}_{\mathrm{WL}}=\mathrm{LN}(1.0,0.316) \\
& \mathrm{T}_{\mathrm{F}, \mathrm{SL} 1}=\mathrm{LN}\left(900^{\circ} \mathrm{F}, 0.412\right) \\
& \mathrm{T}_{\mathrm{F}, \mathrm{SL} 2}=\mathrm{LN}\left(900^{\circ} \mathrm{F}, 0.412\right) \\
& \mathrm{T}_{\mathrm{F}, \mathrm{WL}}=\mathrm{LN}\left(350^{\circ} \mathrm{F}, 0.412\right)
\end{aligned}
$$

With these input values, P-RACE gives a mean point estimate failure probability of 0.0186 for this race cutset. For comparison, manually performing a Monte Carlo analysis (varying the median values of the six input distributions using the assumed systematic uncertainty distributions) yields the distribution of cutset failure probability shown on Figure 14. The mean of this distribution was 0.0188 (for the initial seed assumed and 5,000 trials). (The difference between the two values is due only to sampling uncertainty in the Monte Carlo analysis.)

Thus it can be seen that the user can - if desired - use the P-RACE code as currently configured to compute either point estimate failure probabilities (considering random uncertainties only) or race cutset mean values (considering both random and systematic uncertainties). Development of an extension of P-RACE to automatically compute the full distribution of failure probability (which could be input to an accident sequence uncertainty quantification code such as TEMAC) is in progress. 


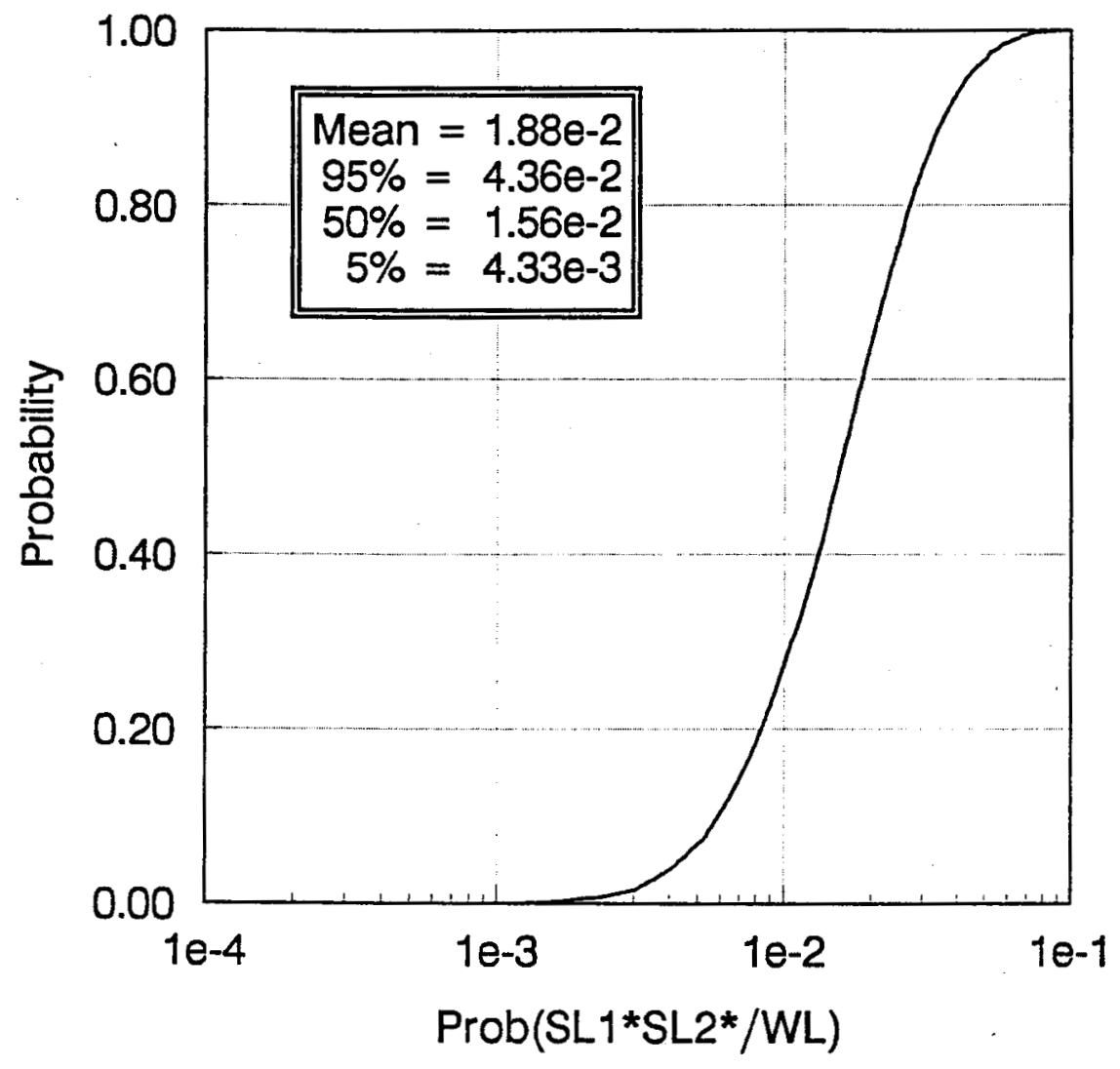

Figure 14 Computed distribution on failure probability 


\subsection{Summary}

The integral formulation presented in this white paper has several advantages over the Monte Carlo formulation:

1. It allows direct and explicit incorporation of random uncertainties both in the failure temperature distributions as well as the responses (i.e., in the code predicted temperature-time histories.)

2. It does not require sampling, and does away with the need to consider sampling uncertainty.

3. It does away with the need to separately derive and characterize the time to failure distributions of the strong and weak links.

4. It can consider arbitrary correlation between the strong link failures (that is, correlation between either the responses of two or more strong links or between their failure temperature distributions.

5. It can still consider deterministic values for failure temperatures (if desired) with no change in formalism.

6. It allows for arbitrary choice of distributions for $T_{S L}, T_{W L}, T_{F, S L}, T_{F, W L}$ and they do not need to be of the same form.

The computer implementation of this formulation is (currently) limited to normal and log normal input distributions, but is quite efficient. An extension to compute distributions of the race cutset failure probabilities is under development. 


\section{Appendix A}

\section{P-RACE USERS GUIDE \\ (Version 5/95)}


The code P-RACE computes the point estimate probability of a race cutset involving several strong links and a single weak link of the form

$$
\operatorname{P}(\operatorname{SL} 1 * \operatorname{SL} 2 * \ldots * / W L)=\operatorname{Prob}\left(t_{S L 1}<t_{W L}, t_{S L 2}<t_{W L}, \ldots\right)
$$

that is, the probability that all the strong links ( $S L_{i}$ ) in the cutset fail before the weak link (WL) fails. This probability is computed using an integral formulation (Equation 6 of the accompanying report) and requires as input the following information for each strong link and the weak 1ink:

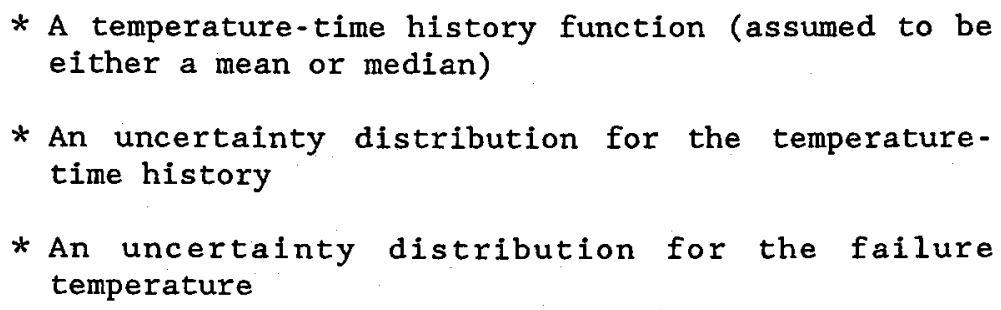

One of several temperature-time functions (quadratic, power law, exponential) can be selected by the user, with coefficients specified in the input. (Each different link can have a different temperature-time history function). An important limitation in this version is that all temperature-time functions must be monotonically increasing. (This limitation could be relaxed, if desired).

The input uncertainty distributions may be either normal or lognormal probability distributions. (The formulation allows for arbitrary distributions to be used and P-RACE could be modified to consider any particular parametric or empirical form, but for most purposes, the normal and lognormal forms will suffice.) only the parameters of the distributions need be input. An important limitation in this version of P-RACE is that the distributional forms of the uncertainties for the temperature response and the failure temperature for any particular link must be the same. (That is, if strong link No. 1 has a normal distribution for its failure temperature uncertainty, then it must also have a normal distribution for its temperature response uncertainty distribution). However, different links may have different uncertainty distributions.

Input is not free fleld, but all numerical input is in fields of 10 .

* The input file must be named P-RACE.INP

* All integers must be right-justified

* Real numbers must have a decimal point somewhere in the field 
The specific lines of input (for each race cutset) are as described below:

Line 1 Title Information (Format 80A1)

Line 2 NSL, NDT, $t_{i}, t_{f}$, Rho- $r$, Rho-f (Format 2I10, 4F10)

where

NSL - Integer number of strong links in cutset (note: one weak link is always assumed, so total number of links in the cutset is $\mathrm{NSL}+1)$

NDT = Integer number of numerical integration increments into which the time interval of integration is divided. Typically, NDT $=50$ is more than adequate.

$t_{i}=$ Initial time (lower limit) for the numerical integration. Theoretically, the integration is from 0.0 to infinity. In practice, any lower limit can be used as long as the contribution to the integral for shorter times is negligible. (A convergence check is included in the output as described below). Provided all temperature-time history functions specified by the user give positive temperatures for times $\geq$ $0.0, t_{i}=0.0$ may be used. (Real number $\geq 0.0$ )

However, if one or more of the user specified temperature-time history functions gives temperatures $\leq 0.0$ for times greater than or equal to zero, then the user has two options:

(a) Use only normal distributions for all links. Then any $t_{i}$ greater than or equal to zero can be used. This is because the normal distribution does not blow up for temperatures $\leq 0.0$ as does the lognormal distribution.

(b) Choose any $t_{i}$ larger than the smallest time beyond which a11 temperatures are $>0.0$.

For any case where the user specifies a $t_{i}>0.0$, the user must verify that $t_{i}$ selected has not omitted any significant portion of the integral, as described below.

$t_{f}=$ Final time (upper limit) for the numerical integration. This can have any value, provided the user verifies that the value is sufficiently large that the integral has converged. (Again, a convergence check is included in the output as described below). (Real, positive number and, of course, $t_{f}$ must be greater than $t$ )

Rho-r = Correlation coefficient between the temperature-time history responses of the first two strong links input. (Real number with $0.0 \leq \rho_{\mathrm{r}} \leq 1.0$ )

Rho-f = Correlation coefficient between the failure temperatures of the first two strong links input. (Real number with $0.0 \leq \rho_{\mathrm{f}} \leq 1.0$ ) 
Line(s) 3 THTYPE, A, B, C (Format I10, 3F10)

where

THTYPE = Integer specifying type of temperature time history to be used for each link.

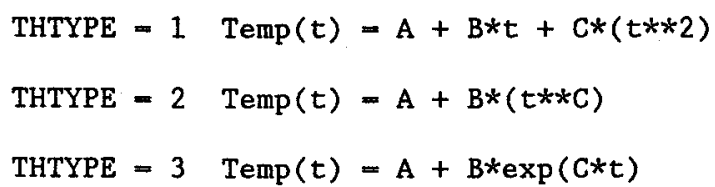

$A, B, C=$ The (real number) coefficients in the selected temperature time history function. The coefficients must be selected such that the function is monotonically increasing.

As mentioned above, if a lognormal distribution is being used for any of the strong links, its corresponding temperature-time history function must give positive, non-zero temperatures for all times in the range $t_{i}$ to $t_{f}$.

Line 3 is repeated for each link, so a total of NSL +1 of these lines are required to specify the temperature-time history functions for all the strong and weak links. THE INPUT FOR THE WEAK LINK MUST ALWAYS BE LISTED FIRST !

Line(s) 4 RTYPE, $M_{r}$, Sig-r (Format I10, 2F10)

where

RTYPE - Type of uncertainty distribution specified for the input temperature-time history response function. (Integer)

$$
\begin{array}{ll}
\text { RTYPE }=2 & \text { Normal distribution } \\
\text { RTYPE }=3 & \text { Lognormal distribution }
\end{array}
$$

$M_{r}=$ Always input as 1.0. This is to remind the user that the uncertainty on the temperature-time history is a multiplicative value, and is input as such below.

Sig-r = Multiplicative dispersion parameter of the distribution (Real, positive number)

Sig-r denotes the coefficient of variation, ( $\operatorname{COV}=\sigma_{\mathrm{r}} / \mu_{\mathrm{r}}$ where $\mu_{r}$ is the mean and $\sigma_{r}$ the standard deviation) if a normal distribution has been specified (RTYPE $=2$ ), or

Sig- $r$ denotes the standard deviation of the logarithms $\left(\beta_{\mathbf{r}}\right)$ if a lognormal distribution has been specified (RTYPE $=3$ )

Line 4 is repeated for each link, so a total of NSL +1 of these lines are required to specify the temperature-time history uncertainty distributions for all the strong and weak links. THE INPUT FOR THE WEAK LINK MUST ALWAYS BE LISTED FIRST! THE ORDER IN WHICH THE STRONG LINK UNCERTAINTY DISTRIBUTIONS ARE INPUT MUST BE THE SAME AS WAS USED IN THE LINE 3 INPUT! 
Line(s) 5 FTYPE, Mf, Sig-f (Format I10, 2F10)

where

FTYPE = Type of uncertainty distribution specified for the failure temperature. (Integer)

\author{
FTYPE $=2$. Normal distribution \\ FTYPE $=3$ Lognormal distribution
}

$M_{f}=\quad \begin{aligned} & \text { The central value parameter of the distribution } \\ & \text { (Real, positive number) }\end{aligned}$

$M_{f}$ denotes the the mean value $\mu_{f}$ (in units of temperature) if a normal distribution has been specified (RTYPE -2 ), or

Mf denotes the the median value $m_{f}$ (in units of temperature) if a lognormal distribution has been specified (RTYPE = 3)

Sig-f $=$ The dispersion parameter of the distribution

(Real, positive number)

Sig-f denotes the standard deviation $\sigma_{f}$ (in units of temperature) if a normal distribution has been specified $($ FTYPE -2$)$, or

Sig-r denotes the standard deviation of the logarithms $\beta_{f}$ (a dimensionless number) if a lognormal distribution has been - specified (FTYPE $=3$ )

Line 5 is repeated for each link, so a total of NSL +1 of these lines are required to specify the failure temperature uncertainty distributions for all the strong and weak links. THE INPUT FOR THE WEAK LINK MUST ALWAYS BE LISTED FIRST! THE ORDER IN WHICH THE STRONG LINK UNCERTAINTY DISTRIBUTIONS ARE INPUT MUST BE THE SAME AS WAS USED IN THE LINE 3 AND LINE 4 INPUT!

\title{
NOTES ON INPUT
}

(a) Each block of data (Lines 1 through 5) applies to a single race cutset, but multiple blocks of data may be input. Do not separate the blocks of data with any blank lines.

(b) At the end of the last block of data input, add a line of blanks (columns 1 to 30 ) to signify the end of the input.

(c) Remember: When normal uncertainty distributions are selected for one or more of the links, the temperature-time history response uncertainty distribution is specified (in Lines 4) in terms of the (dimensionless) COV, while the failure temperature uncertainty distribution is specified (in Lines 5) in terms of the actual standard deviation (with dimensions of temperature)

(By contrast, when lognormal distributions are selected, both response and failure temperature distributions are specified in terms of the dimensionless log standard deviation.) 
(d) As described above, when correlation is specified between either the responses and/or the failure temperatures, the correlation is applied to only the first two strong link components listed in the input (in this version of P-RACE). Usually, the user can tell from the time histories and the capacity distributions which are the critical (i.e., the strongest) strong links which should be correlated (if there is a basis for including correlation). However, the user can always interchange the order in which the strong links are input to determine which pair of strong links maximizes the cutset probability, given the specified correlation.

Note that when correlation is included, the run time is substantially increased, due to the additional integration which must be performed. However, the run time is not increased if either $\rho_{r}=\rho_{f}=0.0$ (the uncorrelated case) or $\rho_{r}=\rho_{f}=1.0$ (the fully correlated case).

\section{Example No, 1: Input and Output}

Figure A-1 illustrates the input file P-RACE.INP for the two example problems given in Section 3.0 of the main report. (The line numbers in parentheses were added to this figure to relate to the input descriptions given above, and are not part of the input file.)

In the first block of input, the data for a race cutset involving one strong link and one weak link is input. Line 1 gives the alphanumeric title information. Line 2 specifies NSL $=1$ (one strong link), NDT $=50$ (fifty integration intervals) and integration from $t_{i}=1.0 \mathrm{sec}$ to $t_{f}=80.0$ sec. Finally, on Line 2, zero correlation is specified $\left(\rho_{r}=0.0\right.$ and $\left.\rho_{f}=0.0\right)$.

The first of Lines 3 specifies that the temperature-time history for the weak link is quadratic (THTYPE = 1) with coefficients $A=0.0, B=70.0$ and $C=0.0$. That is, $\mathrm{T}_{\mathrm{wl}}(t)=70 * t$. The second of Lines 3 specifies that the temperature-time history for the strong link is quadratic (THTYPE $=1$ ) with coefficients $A=0.0, B=60.0$ and $C=0.0$. That is, $T_{s 1}(t)=60 * t$. (Note that the weak link input is always first in the list.)

The first of Lines 4 specifies that the temperature response uncertainty for the weak link is log normal (RTYPE $=3$ ) with median of 1.0 (this is always input as $1.0 !$ ) and a log standard deviation of 0.3 , while the second of Lines 4 specifies that the temperature response uncertainty for the strong link is also log normal (RTYPE = 3) with the same log standard deviation (0.3). (In general, of course, neither the type nor the uncertainty parameter need be the same.)

The first of lines 5 specifies that the failure temperature uncertainty for the weak link is log normal (RTYPE $=3$ ) with median of $350^{\circ} \mathrm{F}$ and a $\log$ standard deviation of 0.4 , while the second of Lines 4 specifies that the failure temperature for the strong link is also log normal (RTYPE $=3$ ) with a median of $900^{\circ} \mathrm{F}$ and the same $\log$ standard deviation $(0.4)$. 


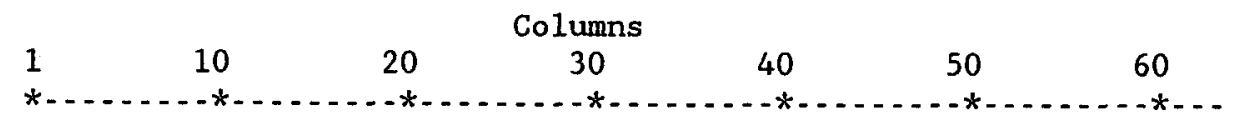

$* * *$ One Strong Link Example Problem (Sect. 3.0 of Main Report)***

(Line 1)

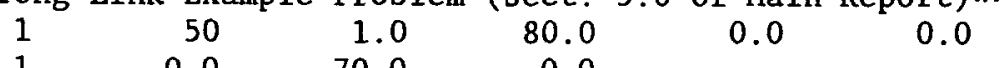

$\begin{array}{lll}0.0 & 70.0 & 0.0\end{array}$

$\begin{array}{lll}0.0 & 60.0 & 0.0\end{array}$

$\begin{array}{ll}1.0 & 0.30\end{array}$

0.0

$\begin{array}{ll}1.0 & 0.30\end{array}$

$\begin{array}{ll}350.0 & 0.40 \\ 900.0 & 0.40\end{array}$

***Two Strong Link Example Problem (Sect. 3.0 of Main Report)***

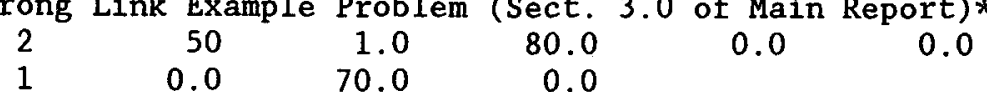

$0.0 \quad 70.0 \quad 0.0$

$\begin{array}{lll}0.0 & 60.0 & 0.0\end{array}$

$\begin{array}{lll}0.0 & 60.0 & 0.0\end{array}$

$1.0 \quad 0.30$

$\begin{array}{ll}1.0 & 0.30\end{array}$

$1.0 \quad 0.30$

$350.0 \quad 0.40$

$900.0 \quad 0.40$

$900.0 \quad 0.40$

(Line 2)

(Line $3 \mathrm{WL}$ )

(Line 3 SLI)

(Line 4 WL)

(Line 4 SL1)

(Line 5 WL)

(Line 5 SL1)

(Line 1)

(Line 2)

(Line $3 \mathrm{WL}$ )

(Line 3 SLI)

(Line 3 SL2)

(Line $4 \mathrm{WL}$ )

(Line 4 SL1)

(Line 4 SL2)

(Line 5 WL)

(Line 5 SL1)

(Line 5 SL2)

Figure A-1 Input File P-RACE. INP for Example No. 1 
This completes the input for the first race cutset and P-RACE could be executed with this input alone (with a line of blanks added to signify the end of the input.) However, for illustrative purposes, the input for a second race cutset has been added so that P-RACE will compute the race cutset probabilities for both in one execution. (There is no limit to the number of race cutset input datasets which may be input at one time.)

In the second block of input, the data for a race cutset involving two strong links and one weak link is input. Line 1 gives the alphanumeric title information. Line 2 specifies NSL -2 (two strong links), NDT $=50$ (fifty integration intervals) and integration from $t_{i}=1.0 \mathrm{sec}$ to $t_{f}=80.0$ sec. Finally, on Line 2, zero correlation is specified $\left(\rho_{\mathrm{r}}=0.0\right.$ and $\left.\rho_{\mathrm{f}}=0.0\right)$.

The first of Lines 3 specifies that the temperature-time history for the weak link is quadratic (THTYPE = 1) with coefficients $\mathrm{A}=0.0, \mathrm{~B}=70.0$ and $\mathrm{C}=0.0$. That is, $T_{\mathrm{wl}}(t)=70 * t$. The next two (of Lines 3 ) specify that the temperature-time histories for the two strong links are quadratic (THTYPE $=1$ ) with coefficients $A=0.0, B=60.0$ and $C=0.0$. That is, $\mathrm{T}_{s 1}(t)=60 * t$ for both strong links. (In general, of course, they need not be the same.)

The first of Lines 4 specifies that the temperature response uncertainty for the weak link is log normal (RTYPE $=3$ ) with median of 1.0 and a $\log$ standard deviation of 0.3 , while the next two (of Lines 4) specify that the temperature response uncertainty for the strong links are also log normal

(RTYPE $=3$ ) with the same log standard deviation (0.3). (In general, of course, neither the type nor the uncertainty parameter need be the same.)

The first of Lines 5 specifies that the failure temperature uncertainty for the weak link is log normal (RTYPE $=3$ ) with median of $350^{\circ} \mathrm{F}$ and a $\log$ standard deviation of 0.4 , while the second two (of Lines 5) specify that the failure temperature for both strong links is also log normal

(RTYPE $=3$ ) with a median of $900^{\circ} \mathrm{F}$ and the same log standard deviation $(0.4)$.

This completes the input for the two race cutsets. One additional line of blanks is added to signify the end of the input file.

The results (always output in file P-RACE.OUT) are shown in Figure A-2. The first block of output is a header which identifies the P-RACE output (and the code version date which was executed).

The next nine lines essentially echo the input for the first race cutset dataset that was input. The input variables are identified by name.

The next block is denoted "Convergence Check" and forms the basis for assuring that the selected values of $t_{i}$ and $t_{f}$ were adequate to capture the 


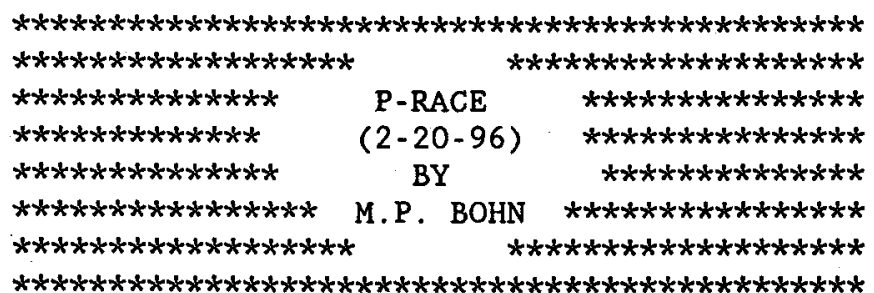

$* * *$ One Strong Link Example Problem (Sect. 3.0 of Main Report) $* * *$ No. SLs 1 No. DTs $=50 t$ (initial) $=1.0 t$ (final) $=80.0$ RHOr $=0.0$ RHOf $=0.0$

T/H No. 1 Quadratic Eqn $A=0.000 \mathrm{E}-01 \quad \mathrm{~B}=7.000 \mathrm{E}+01 \quad \mathrm{C}=0.000 \mathrm{E}-01$ T/H No. 2 Quadratic Eqn A $=0.000 \mathrm{E}-01 \quad B=6.000 \mathrm{E}+01 \quad \mathrm{C}=0.000 \mathrm{E}-01$ Temp Dist No 1 Log Normal Dist Median-r $=1.00$ Beta-r $=0.300$ Temp Dist No 2 Log Normal Dist Median-r $=1.00$ Beta-r $=0.300$ Fail Dist No 1 Log Normal Dist Median-f $=350.00$ Beta-f $=0.400$ Fail Dist No 2 Log Normal Dist Median-f $=900.00$ Beta-f $=0.400$

Convergence Check

Time T-w1 Cdf-SLS Pdf-wl d(Tw1)/dt Del(Pf) Pf

1.790E+00 1.253E+02 1.073E-05 7.717E-04 7.000E+01 9.158E-07 9.158E-07

7.921E+01 5.545E+03 9.996E-01 3.378E-11 7.000E+01 3.735E-09 6.013E-02

$P(R A C E)=6.01 E-02$

***Two Strong Link Example Problem (Sect. 3.0 of Main Report)*** No. SLs 2 No. DTs $=50 t($ initial $)=1.0 t($ final $)=80.0$ RHOr $=0.0$ RHOf $=0.0$

T/H No. 1 Quadratic Eqn $\quad A=0.000 E-01 \quad B=7.000 E+01 \quad C=0.000 E-01$

$\mathrm{T} / \mathrm{H}$ No. 2 Quadratic Eqn $\quad A=0.000 \mathrm{E}-01 \quad \mathrm{~B}=6.000 \mathrm{E}+01 \quad \mathrm{C}=0.000 \mathrm{E}-01$

T/H No. 3 Quadratic Eqn $A=0.000 \mathrm{E}-01 \quad B=6.000 \mathrm{E}+01 \quad \mathrm{C}=0.000 \mathrm{E}-01$

Temp Dist No 1 Log Normal Dist Median-r $=1.00$ Beta-r $=0.300$

Temp Dist No 2 Log Normal Dist Median-r $=1.00$ Beta-r $=0.300$

Temp Dist No 3 Log Normal Dist Median- $r=1.00$ Beta-r $=0.300$

Fail Dist No 1 Log Normal Dist Median-f $=350.00$ Beta-f $=0.400$

Fail Dist No 2 Log Normal Dist Median-f $=900.00$ Beta-f $=0.400$

Fail Dist No 3 Log Normal Dist Median-f $=900.00$ Beta-f $=0.400$

Convergence Check

Time T-w1 Cdf-SLS Pdf-wl d(Tw1)/dt Del(Pf) Pf

1.790E+00 1.253E+02 1.151E-10 7.717E-04 7.000E+01 9.825E-12 9.825E-12

$\begin{array}{lllllll}7.921 \mathrm{E}+01 & 5.545 \mathrm{E}+03 & 9.991 \mathrm{E}-01 & 3.378 \mathrm{E}-11 & 7.000 \mathrm{E}+01 & 3.733 \mathrm{E}-09 & 1.580 \mathrm{E}-02\end{array}$

$P(R A C E)=1.58 \mathrm{E}-02$

Figure A-2 Output File P-RACE.OUT for Example No. 1 
entire integral (which, in principle, is an integral over 0.0 to infinity). Two lines of output are given, corresponding to the integrand quantities evaluated at the midpoint of the first integration interval and of the last integration interval. The times shown in the first column are these two time points. For information and checking purposes, the temperature of the weak link (denoted $T$-wl) is given in the second column. The next column lists the combined cumulative distribution function value of all the strong links (denoted as Cdf-Sls). The column denoted Pdf-wl gives the value of the weak link probability density function, while $d(T w 1) / d t$ denotes the value of the derivative of the weak link temperature-time history at the two time points.

Del(Pf) is the increment added to the integral at each time increment, and is the product

$$
\operatorname{Del}(P f)=(\operatorname{Cdf}-S 1 s) *(P d f-w 1) *[d(T w 1) / d t] *(d t)
$$

Pf is the value of the integral at the end of each time increment. Thus at the end of the first time increment, Pf $=\operatorname{Del}(\mathrm{Pf})$. At the end of the last time increment, $P f=P(R A C E)$, the probability of the race cutset which is listed on the last line of the output for each race input. The integral converges (as $t_{f}$ gets large) because the term Cdf-sls is a probability and approaches 1.0 for large $t_{f}$, while the term Pdf-wl is a probability density function, which approaches zero as $t_{f}$ becomes large.

If the initial time $\left(t_{i}\right)$ specified by the user is sufficiently small, then Pf = Del(Pf) for the first time increment should be very small relative to the final value of the integral, $P(R A C E)$. If the final time $\left(t_{f}\right)$ specified by the user is sufficiently large, $\operatorname{Del}(\mathrm{Pf})$ for the last time increment should be very small relative to the final value of the integral,

Pf $=P(R A C E)$. Thus by examining the values of $\operatorname{Del}(\mathrm{Pf})$ at the end of the first and last integration time increments as given in the "Convergence Check" block of output, the user can be assured that appropriate values of $t_{i}$ and $t_{f}$ were chosen.

The last line of output is, as noted above, the final point estimate value of the probability of the race cutset, P(RACE), a (dimensionless) number between 0.0 and 1.0 .

The output for the next race cutset evaluated follows (in the same format), with the addition of lines echoing the input for the additional strong link.

\section{Example No. 2: Input and Output}

The same two strong link race cutset considered in Example No. 1 is used, except that now two correlation cases will be considered. For the first cutset, partial correlation consisting of 1008 correlation between the computed temperature responses but zero correlation between the failure temperatures is input, i.e.,

$$
\rho_{r}=1.0 \text { and } \rho_{f}=0.0
$$

For the second cutset, both computed temperature responses and failure temperatures are assumed to be fully correlated, i.e., 


$$
\rho_{\mathrm{r}}=1.0 \text { and } \rho_{\mathrm{f}}=1.0
$$

The input file for these two race cutset cases is shown in Figure A-3. The only change from the Example No. 1 input (for the two strong link race cutset shown in Figure $A-1$ ) is on Line 2, where the appropriate values of $\rho_{\mathrm{r}}$ and $\rho_{\mathrm{f}}$ are input.

The output from these two calculations is shown in Figure A-4. It can be seen the the cutset probability has increased from the fully uncorrelated (Example No. 1) value of $1.58 \mathrm{e}-2$ to a value $2.36 \mathrm{e}-02$ for the partially correlated case, and to a value of $6.01 \mathrm{e}-2$ for the fully correlated case.

Example No. 3: Input and Output

Again we shall use the cutsets in Example No. 1, except that the uncertainty distributions shall be specified as normal probability distributions. For comparison purposes, the normal probability distributions will be specified to have the same means and coefficients of variation (c.o.v.) as the log normal distributions used in Example No. 1. The mean and c.o.v. of a lognormal distribution are equal to

$$
\begin{aligned}
& \mu=\mathrm{m} * \exp \left(\beta^{2} / 2\right) \\
& \text { c.o.v }=\sqrt{\exp \left(\beta^{2}\right)-1}
\end{aligned}
$$

Thus for the normal distributions, we will specify

Temperature Response $\mu(\mathrm{WL})=73.22 * t$ and $c .0 . v .(W L)=0.307$
Temperature Response $\mu($ SL1) $=62.76 * t$ and c.o.v. (SL1) $=0.307$
Temperature Response $\mu($ SL2) $=62.76 * t$ and c.o.v.(SL2) $=0.307$

and
Failure Temperature $\mu$ (WL) $=379.2^{\circ} \mathrm{F}$
and $\sigma(\mathrm{WL})=146^{\circ} \mathrm{F}$
Failure Temperature $\mu$ (SL1) $=975.0^{\circ} \mathrm{F}$
and $\sigma($ SL1 $)=375^{\circ} \mathrm{F}$
Failure Temperature $\mu(\mathrm{SL} 2)=975.0^{\circ} \mathrm{F}$
and $\sigma(\mathrm{SL} 2)=375^{\circ} \mathrm{F}$

The same temperature-time histories will be used, and no correlation. Note that (as stated earlier) when a normal distribution is input, the temperature response uncertainty is specified in terms of its c.o.v. while the failure temperature distribution uncertainty is specified in terms of the standard deviation. The input file is shown in Figure A-5, and the corresponding output file in Figure A-6. The probabilities of the two race cutsets (with normal probability distributions) are seen to be $1.56 \mathrm{e}-01$ and $6.56 \mathrm{e}-02$, respectively, and these values are somewhat larger than the values for the lognormal input cases in Example No. 1 (which were 6.01e-02 and $1.58 \mathrm{e}-02$, respectively). This is to be expected, since the normal distribution is symmetric while the lognormal distribution is skewed with longer tails for higher temperatures. Thus, the strong links are less likely to fail before the weak link. (However, if the lognormal uncertainty is small - say, $\beta<0.1$ - the difference between results obtained by using equivalent lognormal and normal distributions is small.) 
$* * *$ Two Strong Link Example Problem (Sect. 3.0 of Main Report)*** (Line 1)

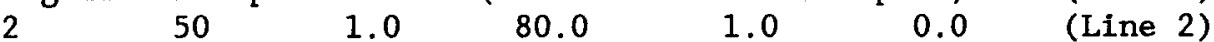

$\begin{array}{lllll}1 & 0.0 & 70.0 & 0.0 & \text { (Line } 3 \mathrm{WL} \text { ) }\end{array}$

$1 \quad 0.0 \quad 60.0 \quad 0.0$

$1 \quad 0.0 \quad 60.0 \quad 0.0$

$3 \quad 1.0 \quad 0.30$

$\begin{array}{ll}1.0 & 0.30\end{array}$

$1.0 \quad 0.30$

$350.0 \quad 0.40$

$900.0 \quad 0.40$

$900.0 \quad 0.40$

***Two Strong Link Example Problem (Sect. 3.0 of Main Report)***

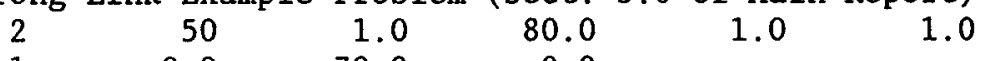

(Line 3 WL)

(Line 3 SL1)

(Line 3 SL2)

(Line $4 \mathrm{WL}$ )

(Line 4 SL1)

(Line 4 SL2)

(Line $5 \mathrm{WL}$ )

(Line 5 SL1)

(Line 5 SL2)

(Line 1)

(Line 2)

(Line $3 \mathrm{WL}$ )

$\begin{array}{lll}0.0 & 60.0 & 0.0\end{array}$

$\begin{array}{lll}0.0 & 60.0 & 0.0\end{array}$

$1.0 \quad 0.30$

$1.0 \quad 0.30$

$\begin{array}{ll}1.0 & 0.30\end{array}$

$350.0 \quad 0.40$

$900.0 \quad 0.40$

$900.0 \quad 0.40$

(Line 3 SL1)

(Line 3 SL2)

(Line $4 \mathrm{WL}$ )

(Line 4 SL1)

(Line 4 SL2)

(Line 5 WL)

(Line 5 SL1)

(Line 5 SL2)

Figure A-3 Example No. 2 Input File P-RACE. INP 


\begin{tabular}{|c|c|c|}
\hline 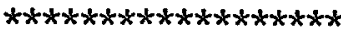 & \multicolumn{2}{|c|}{$\star * * * * * * * * * * * * * * * * * * * *$} \\
\hline$* * * * * * * * * * * * * * *$ & P-RACE & $\star * * * * * * * * * * * * * * * *$ \\
\hline$\star * * * * * * * * * * * * *$ & $(2-20-96)$ & $* \star * * * * * * * * * * * * * * *$ \\
\hline$* * * * * * * * * * * * * * * * *$ & BY & $\star * * * * * * * * * * * * *$ \\
\hline$* * * * * * * * * * * * * * * * * x$ & M.P. BOHN & $\star * * * * * * * * * * * * * * * * * *$ \\
\hline$* * * * * * * * * * * * * * * * * * *$ & & $k * * * * * * * * * * * * * * * * * *$ \\
\hline
\end{tabular}

$* * *$ Two Strong Link Example Problem (Sect. 3.0 of Main Report)*** No. SLs 2 No. DTs $=50 t$ (initial) $=1.0 t$ (final $)=80.0$ $\mathrm{RHOr}=1.0$ RHOF $=0.0$

T/H No. 1 Quadratic Eqn $\quad A=0.000 E-01 \quad B=7.000 E+01 \quad C=0.000 E-01$ $\mathrm{T} / \mathrm{H}$ No. 2 Quadratic Eqn $A=0.000 \mathrm{E}-01 \quad \mathrm{~B}=6.000 \mathrm{E}+01 \quad \mathrm{C}=0.000 \mathrm{E}-01$

T/H No. 3 Quadratic Eqn $A=0.000 E-01 \quad B=6.000 E+01 \quad C=0.000 E-01$ Temp Dist No 1 Log Normal Dist Median-r $=1.00$ Beta-r $=0.300$ Temp Dist No 2 Log Normal Dist Median-r = 1.00 Beta-r $=0.300$ Temp Dist No 3 Log Normal Dist Median-r $=1.00$ Beta-r $=0.300$ Fail Dist No 1 Log Normal Dist Median-f $=350.00$ Beta-f $=0.400$ Fail Dist No 2 Log Normal Dist Median-f $=900.00$ Beta-f $=0.400$ Fail Dist No 3 Log Normal Dist Median-f $=900.00$ Beta-f $=0.400$

Convergence Check

Time T-wl Cdf-SLS Pdf-wl d(Tw1)/dt Del(Pf) Pf

$\begin{array}{lllllll}1.790 \mathrm{E}+00 & 1.253 \mathrm{E}+02 & 3.514 \mathrm{E}-08 & 7.717 \mathrm{E}-04 & 7.000 \mathrm{E}+01 & 3.000 \mathrm{E}-0.9 & 3.000 \mathrm{E}-09\end{array}$

$\begin{array}{lllllll}7.921 \mathrm{E}+01 & 5.545 \mathrm{E}+03 & 9.991 \mathrm{E}-01 & 3.378 \mathrm{E}-11 & 7.000 \mathrm{E}+01 & 3.733 \mathrm{E}-09 & 2.363 \mathrm{E}-02\end{array}$

$P(R A C E)=2.36 E-02$

***Two Strong Link Example Problem (Sect. 3.0 of Main Report)*** No. SLs 2 No. DTs $=50 t$ (initial $)=1.0 t$ (final $)=80.0$ RHOr $=1.0$ RHOf $=1.0$

T/H No. 1 Quadratic Eqn $A=0.000 E-01 \quad B=7.000 E+01 \quad C=0.000 E-01$

T/H No. 2 Quadratic Eqn $A=0.000 \mathrm{E}-01 \quad B=6.000 \mathrm{E}+01 \quad \mathrm{C}=0.000 \mathrm{E}-01$

T/H No. 3 Quadratic Eqn $A=0.000 E-01 \quad B=6.000 E+01 \quad C=0.000 E-01$

Temp Dist No 1 Log Normal Dist Median-r $=1.00$ Beta-r $=0.300$

Temp Dist No 2 Log Normal Dist Median-r $=1.00$ Beta-r $=0.300$

Temp Dist No 3 Log Normal Dist Median-r $=1.00$ Beta-r $=0.300$

Fail Dist No 1 Log Normal Dist Median-f $=350.00$ Beta-f $=0.400$

Fail Dist No 2 Log Normal Dist Median-f $=900.00$ Beta-f $=0.400$

Fail Dist No 3 Log Normal Dist Median-f $=900.00$ Beta-f $=0.400$

Convergence Check

Time T-wl Cdf-SLS Pdf-wl d(Twl)/dt Del(Pf) Pf

$\begin{array}{llllllll}1.790 \mathrm{E}+00 & 1.253 \mathrm{E}+02 & 1.073 \mathrm{E}-05 & 7.717 \mathrm{E}-04 & 7.000 \mathrm{E}+01 & 9.158 \mathrm{E}-07 & 9.158 \mathrm{E}-07\end{array}$

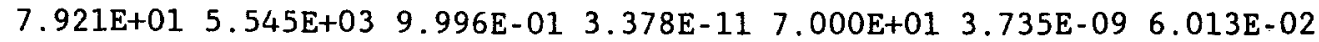

$\mathrm{P}(\mathrm{RACE})=6.01 \mathrm{E}-02$

Figure A-4 Output file P-RACE. OUT for Example No. 2 


\begin{tabular}{|c|c|c|c|c|c|c|c|}
\hline \multicolumn{3}{|c|}{$* * * * * * * * * * * * * * * * * * * * * * * * *$ Example } & \multicolumn{3}{|c|}{$3 * * * * * * * * * * * * * * * * * * * * * * * * * * * * * * *$} & \multicolumn{2}{|c|}{ (Line 1) } \\
\hline 1 & 50 & 1.0 & 80.0 & 0.0 & 0.0 & (Line & \\
\hline 1 & 0.0 & 73.22 & 0.0 & & & (Line & 3 WL) \\
\hline 1 & 0.0 & 62.76 & 0.0 & & & (Line & 3 SL1) \\
\hline 2 & 1.0 & 0.307 & & & & (Line & 4 WL) \\
\hline 2 & 1.0 & 0.307 & & & & (Line & 4 SL1) \\
\hline 2 & 379.2 & 146.0 & & & & (Line & 5 WL) \\
\hline 2 & 975.0 & 375.0 & & & & (Line & 5 SL1) \\
\hline$* * * * * * * * *$ & $* * * * * * * * x$ & *Exampl & $3 * * * * * * * * *$ & $* * * * x+2$ & $* * * * * * * * x$ & (Line & 1) \\
\hline 2 & 50 & 1.0 & 80.0 & 0.0 & 0.0 & (Line & 2) \\
\hline 1 & 0.0 & 73.22 & 0.0 & & & (Line & 3 WL) \\
\hline 1 & 0.0 & 62.76 & 0.0 & & & (Line & 3 SL1) \\
\hline 1 & 0.0 & 62.76 & 0.0 & & & (Line & 3 SL2) \\
\hline 2 & 1.0 & 0.307 & & & & (Line & 4 WL) \\
\hline 2 & 1.0 & 0.307 & & & & (Line & 4 SL1) \\
\hline 2 & 1.0 & 0.307 & & & & (Line & 4 SL2) \\
\hline 2 & 379.2 & 146.0 & & & & (Line & 5 WL) \\
\hline 2 & 975.0 & 375.0 & & & & (Line & 5 SL1) \\
\hline 2 & 975.0 & 375.0 & & & & (Line & 5 SL2) \\
\hline
\end{tabular}




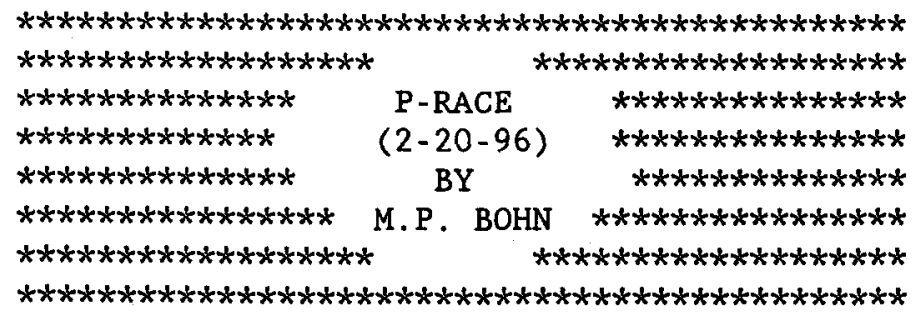

$* * * * * * * * * * * * * * * * * * * * * *$ Example $3 * * * * * * * * * * * * * * * * * * * * * * * * * * * * * * * * * * * *$ No. SLs 1 No. DTs $=50$ t(initial $)=1.0 t$ (final $)=80.0$ RHOr $=0.0$ RHOf $=0.0$

$\mathrm{T} / \mathrm{H}$ No. 1 Quadratic Eqn $\mathrm{A}=0.000 \mathrm{E}-01 \quad \mathrm{~B}=7.322 \mathrm{E}+01 \quad \mathrm{C}=0.000 \mathrm{E}-01$

T/H No. 2 Quadratic Eqn $A=0.000 \mathrm{E}-01 \quad B=6.276 \mathrm{E}+01 \quad \mathrm{C}=0.000 \mathrm{E}-01$

Temp Dist No 1 Normal Dist Mean-r $=1.00$ COV-r $=0.31$

Temp Dist No 2 Normal Dist Mean- $r=1.00$ COV-r $=0.31$

Fail Dist No 1 Normal Dist Mean-f $=379.20$ Sigma-f $=146.00$

Fail Dist No 2 Normal Dist Mean-f $=975.00$ Sigma-f $=375.00$

Convergence Check

Time T-w1 Cdf-SLS Pdf-w1 d(Twl)/dt Del(Pf) Pf

$\begin{array}{lllllll}1.790 E+00 & 1.311 E+02 & 1.100 \mathrm{E}-02 & 6.882 \mathrm{E}-04 & 7.322 \mathrm{E}+01 & 8.757 \mathrm{E}-04 & 8.757 \mathrm{E}-04\end{array}$

$\begin{array}{lllllll}7.921 \mathrm{E}+01 & 5.800 \mathrm{E}+03 & 9.945 \mathrm{E}-01 & 2.238 \mathrm{E}-06 & 7.322 \mathrm{E}+01 & 2.575 \mathrm{E}-04 & 1.564 \mathrm{E}-01\end{array}$

$P($ RACE $)=1.56 \mathrm{E}-01$

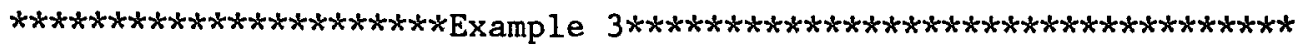

No. SLs 2 No. DTs $=50 t$ (initial) $=1.0 t$ (final $)=80.0$

RHOr $=0.0$ RHOf $=0.0$

T/H No. 1 Quadratic Eqn $A=0.000 \mathrm{E}-01 \quad B=7.322 \mathrm{E}+01 \quad C=0.000 \mathrm{E}-01$

$\mathrm{T} / \mathrm{H}$ No. 2 Quadratic Eqn $\mathrm{A}=0.000 \mathrm{E}-01 \quad \mathrm{~B}=6.276 \mathrm{E}+01 \quad \mathrm{C}=0.000 \mathrm{E}-01$

T/H No. 3 Quadratic Eqn $A=0.000 \mathrm{E}-01 \quad B=6.276 \mathrm{E}+01 \quad \mathrm{C}=0.000 \mathrm{E}-01$

Temp Dist No 1 Normal Dist Mean-r $=1.00$ COV-r $=0.31$

Temp Dist No 2 Normal Dist Mean-r $=1.00$ COV-r $=0.31$

Temp Dist No 3 Normal Dist Mean-r $=1.00$ COV-r $=0.31$

Fail Dist No 1 Normal Dist Mean-f $=379.20$ Sigma-f $=146.00$

Fail Dist No.2 Normal Dist Mean-f $=975.00$ Sigma-f $=375.00$

Fail Dist No 3 Normal Dist Mean-f $=975.00$ Sigma-f $=375.00$

Convergence Check

Time T-wl Cdf-sLS Pdf-wl $d(T w 1) / d t$ Del(Pf) Pf

$\begin{array}{lllllll}1.790 \mathrm{E}+00 & 1.311 \mathrm{E}+02 & 1.210 \mathrm{E}-04 & 6.882 \mathrm{E}-04 & 7.322 \mathrm{E}+01 & 9.631 \mathrm{E}-06 & 9.631 \mathrm{E}-06\end{array}$

$\begin{array}{lllllll}7.921 \mathrm{E}+01 & 5.800 \mathrm{E}+03 & 9.890 \mathrm{E}-01 & 2.238 \mathrm{E}-06 & 7.322 \mathrm{E}+01 & 2.560 \mathrm{E}-04 & 6.555 \mathrm{E}-02\end{array}$

$P(R A C E)=6.56 \mathrm{E}-02$

Figure A-6 Example No. 3 Output file P-RACE.OUT 


\section{Appendix II: \\ P-RACE: A Program for Calculating \\ Probabilities of Strong Link/Weak Link Cutsets}

by

M.P. Bohn

February 20, 1996

This appendix contains a previously unpublished program by M.P. Bohn for the quantification of the probability of failure of temperature-dependent weak link/strong link systems. 


\section{PROGRAM PRACE}

C THIS PROGRAM COMPUTES THE POINT ESTIMATE PROBABIIITY

C

C

C

C

C

C

C

C

C

C

C

C

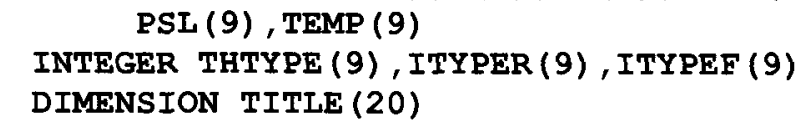

C

WRITE HEADER

WRITE $(6,500)$

500

* FORMAT (1HO, 13$)$

$\star$

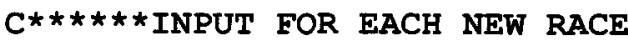

$999 \operatorname{READ}(5,1000)$ (TITLE (I), I=1, 20)

WRITE $(6,1001)$ (TITLE (I), I=1, 20)

1000 FORMAT (20A4)

1001 FORMAT (1H0, 4X, 20A4)

C READ IN NUMBER OF INTEGRATION INCREMENTS AND

C UPPER LIMIT INTEGRATION TIME

1005 FORMAT $(2 \mathrm{I} 10,4 \mathrm{~F} 10.2)$

IF (NSL .EQ. 0) GO TO 200

WRITE $(6,1)$ NSL , NDT , TI , TF, RHOR, RHOF

1 FORMAT (5X,' NO. SLs', I2, $2 \mathrm{X},{ }^{\prime}$ NO. DTs $=1, I 5,2 \mathrm{X},{ }^{\prime} t$ (initial) $=$ ',

$\star$ F7.1,2X,'t(final $)=', F 7.1,2 \mathrm{X}, 1,5 \mathrm{X},{ }^{\prime}$ RHOr $=', \mathrm{~F} 4.1,2 \mathrm{X},{ }^{\prime}$ RHOf $=$ ',

* F4.1)

C

READ IN WL, SL'S, TEMPERATURE-TIME CURVES

**NOTE** WL DATA IS ALWAYS INPUT FIRST***

$\operatorname{READ}(5,2)$ ( $\operatorname{THTYPE}(I), A(I), B(I), C(I), I=1, N S L+1)$ 
C

4

50

51

52

53

C

C

C

C

C

C $\star$
*

$\star$

DO $36 I=1, N S L+1$

IF (THTYPE (I) .EQ. 1) WRITE $(6,33)$ I, A(I) , B (I) ,C (I)

IF (THTYPE (I) .EQ. 2) WRITE $(6,34)$ I,A (I), B(I),C (I)

IF (THTYPE (I) .EQ. 3) WRITE $(6,35)$ I, A(I) , B (I)

FORMAT (5X,' T/H No.' ,I2, 2X,' Quadratic EqM $', 2 \mathrm{X},{ }^{\prime} \mathrm{A}=$ ', $1 \mathrm{P}$ E10.3,

FORMAT (5X,'T/H No.' ,I2, 2X,' Power Law Eqn ', $2 \mathrm{X},{ }^{\prime} \mathrm{A}=1,1 \mathrm{P}$ E10.3, $2 \mathrm{X},{ }^{\prime} \mathrm{B}={ }^{\prime}, 1 \mathrm{P} \mathrm{E} 10.3,2 \mathrm{X},{ }^{\prime} \mathrm{C}=1,1 \mathrm{P} \mathrm{E} 10.3$ )

FORMAT (5X,'T/H No.' , I2, 2X,' Exponential Eqn', 2X,'A =', 1P E10.3,

* $2 \mathrm{X},{ }^{\prime} \mathrm{B}={ }^{\prime}, 1 \mathrm{P} \mathrm{E} 10.3$ )

CONTINUE

READ IN TEMPERATURE RESPONSE UNCERTAINTY DISTRIBUTIONS

$\operatorname{READ}(5,4) \quad(\operatorname{ITYPER}(I), \operatorname{MR}(I), \operatorname{SIGR}(I), I=1, \mathrm{NSL}+1$ )

FORMAT (I10, 2F10.2)

DO $53 \mathrm{I}=1, \mathrm{NSL}+1$

$\operatorname{IF}(\operatorname{ITYPER}(I)$.EQ. 1) WRITE $(6,50)$ I,MR(I), SIGR(I)

FORMAT (5X,' Temp Dist No', I2, 2X,' Uniform Dist ',2X,

'Tlower =' ,F8.2,2X,' Tupper =',F8.2)

IF( ITYPER(I) .EQ. 2) WRITE $(6,51) \quad I, M R(I), \operatorname{SIGR(I)~}$

FORMAT (5X,' Temp Dist No' , I2, 2X,' Normal Dist ', 2X,

'Mean-r =', F8.2, 2X,' COV-r=',F8.2)

IF( ITYPER(I) .EQ. 3) WRITE $(6,52)$ I,MR(I), SIGR(I)

FORMAT (5X,' Temp Dist No' , I2 ,2X,' Log Normal Dist' ,2X,

'Median-r =',F8.2,2X,'Beta-r =', F9.3)

CONTINUE

READ IN FAILURE TEMPERATURE UNCERTAINTY DISTRIBUTIONS

$\operatorname{READ}(5,4) \quad(\operatorname{ITYPEF}(I), \operatorname{MF}(I), \operatorname{SIGF}(I), I=1, N S L+1)$

DO $57 \mathrm{I}=1$, NSL+1

IF ( ITYPEF (I) .EQ. 1) WRITE $(6,54)$ I,MF (I), $\operatorname{SIGF(I)~}$

FORMAT (5X,' Fail Dist No' ,I2, 2X,' Uniform Dist ', 2X,

(1)

IF ( ITYPEF (I) .EQ. 2) WRITE $(6,55)$ I,MF(I), SIGF(I)

FORMAT (5X,' Fail Dist No', I2, 2X,' Normal Dist ',2X,

* 'Mean-f =',F8.2,2X,'Sigma-f =',F8.2)

IF ( ITYPEF (I) .EQ. 3). WRITE $(6,56)$ I, MF (I), SIGF (I)

FORMAT (5X,' Fail Dist No', I2, 2X,' Log Normal Dist' ,2X,

* 'Median-f =',F8.2,2X,'Beta-f =', F9.3)

CONTINUE

MAKE SURE TEMP RESPONSE AND FAIL TEMP DISTRIBUTIONS

MATCH

DO 58 I $=1$, NSL +1

IF（ITYPER(I) .NE.ITYPEF(I) ) WRITE $(6,300)$

IF ( ITYPER(I) .NE.ITYPEF(I) ) STOP

58 CONTINUE

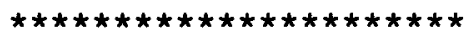

INTEGRATE OVER 0 TO TF

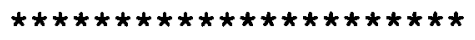

PF $=0.0$

$\mathrm{RDT}=\mathrm{NDT}$
} 


$$
\begin{aligned}
& \mathrm{DT}=(\mathrm{TF}-\mathrm{TI}) / \mathrm{RDT} \\
& \mathrm{T}=\mathrm{TI}-\mathrm{DT} / 2.0 \\
& \mathrm{~S} 22 \mathrm{PI}=2.5066 \\
& \mathrm{DO} 20 \mathrm{~J}=1, \mathrm{NDT} \\
& \mathrm{T}=\mathrm{T}+\mathrm{DT}
\end{aligned}
$$

C

C

C

6

C

C

C

C

C

C

C

C

C

C

C

C

C

C

C

C

COMPUTE TEMPERATURES AT EACH TIME T

DO $6 I=1$,NSL+1

IF (THTYPE (I) .EQ. 1 ) TEMP (I) $=A(I)+B(I) * T+C(I) *(T * * 2)$

IF (THTYPE (I) .EQ. 2 ) TEMP (I) $=A(I)+B(I) *\left((T-T I) *{ }^{2}(I)\right)$

CONTINUE

\begin{abstract}
EVALUATE INTEGRAND

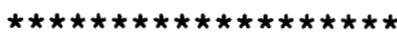

COMPUTE WEAK LINK PDF ORDINATE

LOG NORMAL DISTRIBUTION

IF (ITYPER (1) .EQ. 3 .AND. ITYPEF (1) .EQ. 3 ) THEN

BETA $=\operatorname{SQRT}(\operatorname{SIGR}(1) * * 2+\operatorname{SIGE}(1) * * 2)$

ARG $=-((\operatorname{ALOG}($ TEMP $(1) / M F(1))) * \star 2) /(2.0 *$ BETA $* \star 2)$

IF (ARG . GE. -25 . ) PDEWL $=\operatorname{EXP}(A R G) /($ SQ2PI $* B E T A * T E M P(1))$

IF (ARG . LT. -25. ) PDFWL $=0.0$

ENDIF

NORMAL DISTRIBUTION

IF (ITYPER (1) .EQ. 2 .AND. ITYPEF(1) .EQ. 2 ) THEN

SIGMA $=\operatorname{SQRT}(\operatorname{SIGF}(1) * \star 2+(\operatorname{SIGR}(1) * \operatorname{TEMP}(1)) * \star 2)$

ARG $=-((\operatorname{TEMP}(1)-\mathrm{MF}(1)) * \star 2) /(2.0 *$ SIGMA $* * 2)$

IF (ARG .GE. -25.) PDFWL $=\operatorname{EXP}($ ARG) $/($ SQ2PI*SIGMA)

IF (ARG .IT. -25.) $\quad$ PDFWL $=0.0$

ENDIF

COMPUTE WEAK LINK UNIFORM PDF ORDINATE

***TO BE ADDED***

COMPUTE STRONG IINK PROBABILITIES

DO $10 I=2, N S L+1$

LOG NORMAL DISTRIBUTION

IF (ITYPER (I) .NE. 3 . AND. ITYPEF(I) .NE. 3 ) GO TO 7

NOTATION FOR LOG NORMAL INPUT

MF IS MEDIAN OF FAILURE DISTRIBUTION

SIGF IS BETA-FR OF FAILURE DISTRIBUTION

MR IS MEDIAN OF RESPONSE DISTRIBUTION (1.0)

SIGR IS BETA-RR OF RESPONSE DISTRIBUTION

COMPUTE STRONG LINK CDF FAILURE PROBABILITIES 
$\mathrm{Z}(\mathrm{I})=\operatorname{ALOG}(\operatorname{TEMP}(\mathrm{I}) / \mathrm{MF}(\mathrm{I})) / \operatorname{SQRT}(\mathrm{SIGF}(\mathrm{I}) \star \star 2+\operatorname{SIGR}(\mathrm{I}) \star \star 2)$

PSL $(I)=$ FNO1(Z(I))

IF (I.EQ. 3) THEN

$\mathrm{RHO}=(\mathrm{RHOR} * \mathrm{SIGR}(2) * \mathrm{SIGR}(3)+\mathrm{RHOF} * \mathrm{SIGF}(2) * \mathrm{SIGF}(3)) /$

(SQRT (SIGR $(2) * \star 2+\operatorname{SIGF}(2) * * 2) * \operatorname{SQRT}(\operatorname{SIGR}(3) * \star 2+\operatorname{SIGF}(3) \star \star 2)$ )

PSL12 = FBN01 $(\mathrm{z}(2), \mathrm{z}(3)$, RHO $)$

ENDIF

GO TO 10

C

C

C

C

C

C
NORMAL DISTRIBUTION

CONTINUE

IF (ITYPER (I) .NE. 2 . AND. ITYPEF(I) .NE. 2 ) GO TO 8 NOTATION FOR NORMAL INPUT

MF IS MEAN OF FAILURE DISTRIBUTION

SIGF IS SIGMA-FR OF SL FAILURE DISTRIBUTION

MR IS MEAN OF RESPONSE DISTRIBUTION (1.0)

SIGR IS C.O.V. OF SL RESPONSE DISTRIBUTION

COMPUTE STRONG LINK CDF FAILURE PROBABILITIES

$\mathrm{Z}(I)=(\operatorname{TEMP}(I)-\mathrm{MF}(I)) / \operatorname{SQRT}(\operatorname{SIGF}(I) \star \star 2+(\operatorname{SIGR}(I) \star T E M P(I)) \star \star 2)$

$\operatorname{PSI}(I)=$ FN01( $(\mathrm{Z}(\mathrm{I}))$

IF (I.EQ. 3) THEN

**THIS NOT RIGHT YET WITH SHIFT FACTORS !!!**

RHO $=($ RHOR*SIGR (2) *TEMP (2) *SIGR (3) *TEMP (3) $+\mathrm{RHOF}^{\star}$ SIGF (2) *SIGF (3))/

( SQRT (SIGF (2) **2+(SIGR (2)*TEMP (2)) * 2 ) * SQRT (SIGF (3) * $2+(\operatorname{SIGR}(3) \star \operatorname{TEMP}(3)) \star \star 2)$ )

PSL12 = FBN01 (Z (2), Z (3), RHO)

ENDIF

GO TO 10

UNIFORM DISTRIBUTION

CONTINUE

IF (ITYPER (I) .NE . 1 .AND. ITYPEF(I) .NE. 1) $\operatorname{WRITE~}(6,100)$

IF (ITYPER (I) .NE. I .AND. ITYPEF (I) .NE . 1) STOP

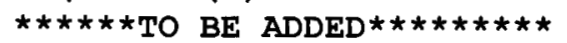

CONTINUE

END OF LOOP OVER STRONGLINKS

COMPUTE DERIVATIVE OF WEAK LINK TEMP-TIME REIATION

QUADRATIC TIME HISTORY

IF (THTYPE (1) .EQ. 1 ) DTEMPDT $=B(1)+2.0 * C(1) \star T E M P(1)$

POWER CURVE TIME HISTORY

IF (THTYPE (1) .EQ. 2 ) DTEMPDT $=\mathrm{C}(1){ }_{\mathrm{B}}(1) *((T-T I) * \star(C(1)-1.0))$ 


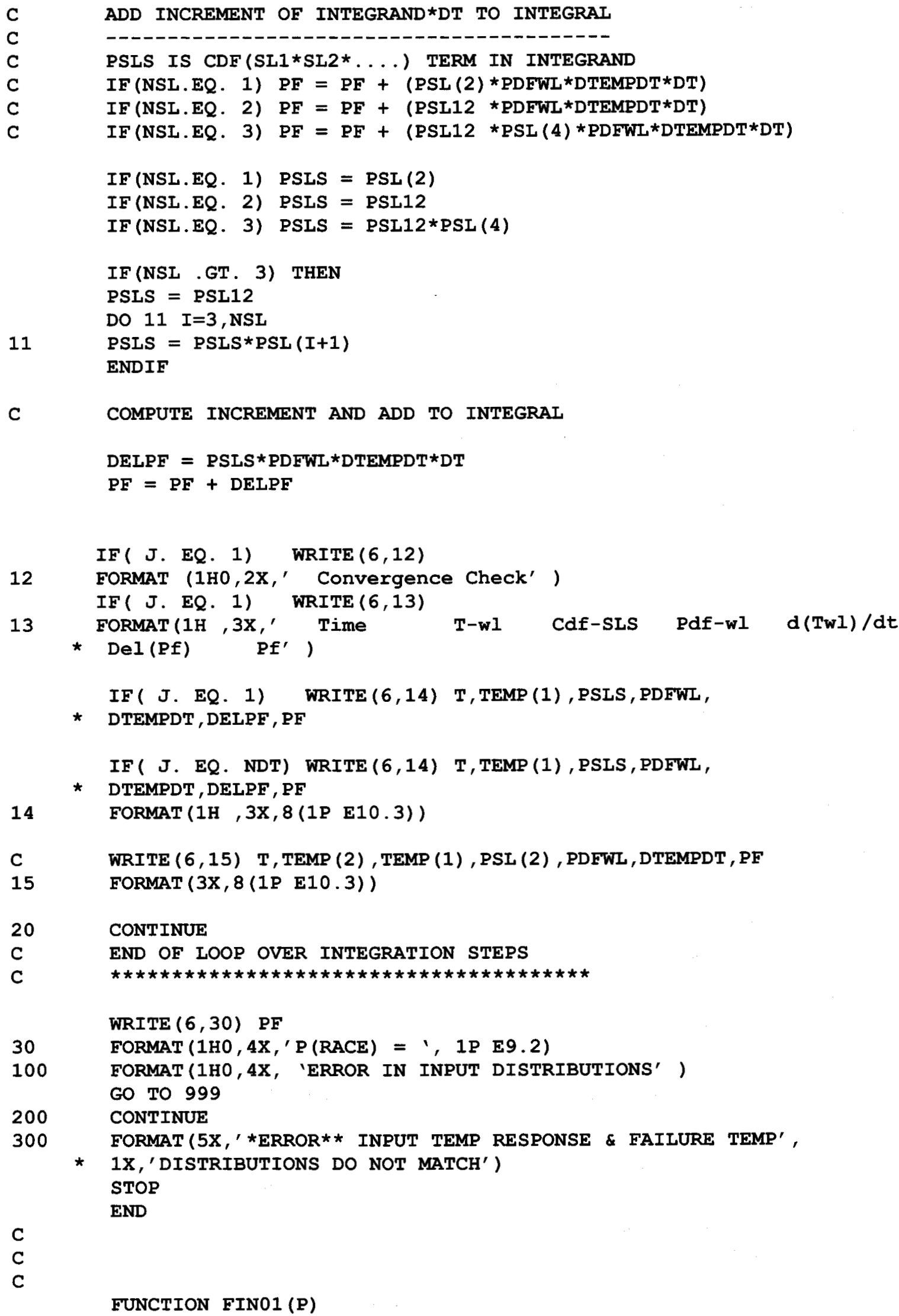




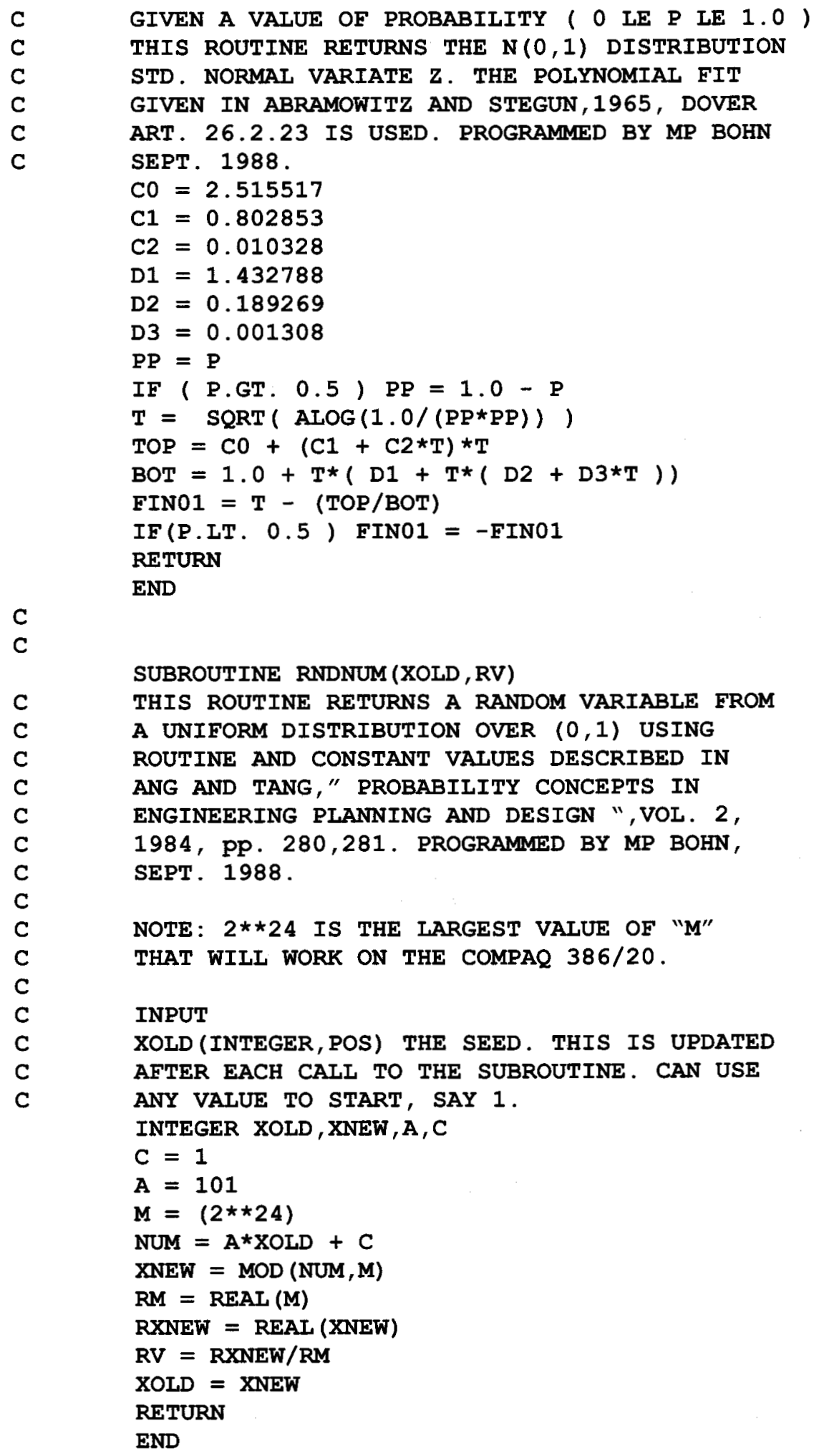

C

FUNCTION FNO1 ( $\mathrm{z})$

C THIS FUNCTION RETURNS THE CDF OF THE NORMAL N $(0,1)$ DISTRIBUTION

C GIVEN THE STD. NORMAL VARIATE $Z$. THE POLYNOMIAL FIT 
C GIVEN IN ABRAMOWITZ AND STEGUN, 1965, DOVER

C ART. 26.2.16 IS USED. PROGRAMMED BY MP BOHN

C SEPT. 1988 .

$$
\begin{aligned}
& \mathrm{A} 1=.4362 \\
& \mathrm{~A} 2=-.1202 \\
& \mathrm{~A} 3=.9373 \\
& \mathrm{P}=.3327
\end{aligned}
$$

C

C

C

C

C

C

C

C

C

C

C

C

c

C

C

C

C

C

C

C

4

C

C

C

1

C IIMITING CASES

IF (RO . GT. 0.02 . AND. RO . IT. 0.98$)$ GO TO 2

$$
\begin{aligned}
& \mathrm{A} 1=0.4361836 \\
& \mathrm{~A} 2=-0.1201676 \\
& \mathrm{~A} 3=0.9372980 \\
& \mathrm{P}=0.33267
\end{aligned}
$$

TO PREVENT TOO SMALI ARGUMENT GOING INTO FUNCTION EXP IF ( $\mathrm{Z} . \mathrm{IT} .-6.36) \mathrm{Z}=-6.36$

IF ( $\mathrm{z} . \mathrm{GT} .+6.36) \mathrm{z}=+6.36$

$T=1.0 /(1.0+P * A B S(Z))$

SQT2PI $=2.506628$

$\mathrm{ZZ}=(\operatorname{EXP}(-\mathrm{Z} * \mathrm{Z} / 2.0)) / \operatorname{SQT} 2 \mathrm{PI}$

FN01 $=1.0-Z Z *(A 1+A 2 * T+A 3 * T * T) * T$

IF (Z.GE. 0.0) RETURN

FNO1 = $1.0-$ FNO1

RETURN

END

FUNCTION FBNO1 (RH, RK, RO) RK ARE THE STANDARD NORMAL VARIATES, AND RO IS THE $T(H, A H)$ AND ENO1 (Z).

FORMULATION IS GIVEN IN ??? PROGRAMMED BY MP BOHN, OCTOBER, 1988. MODIFIED BY MP BOHN, FEBRUARY 1996.

WRITE $(6,4)$ RH, RK, RO FORMAT (20X，3F10.3)

PUT IIMITS ON STANDARD VARIATES TO PREVENT EXCESSIVELY LARGE ARGUMENTS IN FN01() AND T().

IF (RH.LT . - 6.36) RH $=-6.36$

IF (RK.LT. -6.36$) \mathrm{RK}=-6.36$

IF (RH.GT. 6.36) $\mathrm{RH}=6.36$

IF(RK.GT. 6.36) $\mathrm{RK}=6.36$ IF (RO . LT. 0.0) WRITE $(6,1)$

IF (RO .LT. 0.0$)$ STOP

FORMAT (5X,' ERROR - NEGATIVE CORRELATION COEFFICIENT')

THIS FUNCTION COMPUTES THE JOINT BI-VARIATE PROBABILITY $\mathrm{P}(\mathrm{x}<\mathrm{H}, \mathrm{y}<\mathrm{K}, \mathrm{RO})$ USING THE T-INTEGRAI FORMULATION. RH AND CORRELATION COEFFICIENT. THIS FUNCTION CALLS EUNCTIONS

CODE NOT CHECKED OUT FOR NEGATIVE CORRELATION COEFFICIENT 
IF (RO .LE. 0.02) FBN01 $=$ FN01 (RH) $*$ FN01 (RK)

IF (RO .GE . 0.98) FBNO1 $=\operatorname{AMIN1}($ FN01 (RH), ENO1 (RK))

RETURN

2

C

C

C

C

C

C

C

C

C

C

$\mathrm{C}$

C

C

C

C

10

C

C

CONTINUE

COMPUTE $T(-)$ FUNCTION PARAMETERS

RH AND/OR RK CANNOT EQUAL 0.0 OR

AH, AK WILL BLOW UP.

IF (ABS (RH) .LT. 0.00001$) \mathrm{RH}=0.00001 * \operatorname{SIGN}(1.0, \mathrm{RH})$

IF (ABS (RK) .LT. 0.00001 ) $R K=0.00001 * \operatorname{SIGN}(1.0, R K)$

THESE LIMITING VALUES DID NOT AFFECT ACCURACY OF RESULT TO THREE SIGNIFICANT FIGURES IN CHECKOUT.

$\mathrm{AH}=(\mathrm{RK}-\mathrm{RH} * \mathrm{RO}) /(\mathrm{RH} * \mathrm{SQRT}(1.0-\mathrm{RO} * \mathrm{RO}))$

$A K=(R H-R K \star R O) /(R K \star S Q R T(1.0-R O \star R O))$

APPROXIMATE CALCULATION OF BIVARIATE PROBABILITY

WHEN ONE OR MORE OF THE MARGINAL PROBABILITIES

IS LESS THAN 7E-4.

IF (RH .GT. -3.2 .AND. RK .GT. -3.2 ) GO TO 10

$\mathrm{P} 1=\mathrm{FN01}(\mathrm{RH})$

$\mathrm{P2}=\mathrm{FNO1}(\mathrm{RK})$

IF( RO .LT. 0.25 ) FBNO1 $=\mathrm{P} 1 * \mathrm{P} 2$

IF( RO .GE. 0.25 and. RO .LT. 0.75 ) FBNO1 = (P1*P2)**0.75

IF( RO .GE. 0.75 ) FBN01 $=\operatorname{AMIN1}(\mathrm{P} 1, \mathrm{P} 2)$

RETURN

CONTINUE

GENERAL EXPRESSION FOR BIVARIATE PROBABILITY

FUNCTION B (RH, RK, RO)

$\mathrm{CC}=-0.5$

$\mathrm{IF}(\mathrm{RH} * \mathrm{RK} . \mathrm{GT} .0 .0) \mathrm{CC}=0.0$

$I F(R H \star R K$.EQ. 0.0 .AND. $R H+R K . G E, 0.0) C C=0.0$

*

FBN01 $=($ FNO2 (RH) + FNO2 (RK) $) / 2.0$

$-T(R H, A H)-T(R K, A K)+C C$

RETURN

END

C

C

FUNCTION T $(H, A)$

C SIMPSON'S RULE FOR INTEGRATION IS USED TO EVALUATE INTEGRAL

C FOR T(H,A) WITH M=301 INTEGRATION POINTS, BUT COULD USE

C ANY ODD VALUE FOR M. PROGRAMMED BY MP BOHN, OCTOBER 1988.

C 
$M=301$

C

C USE RELATIONS $T(-H, A)=+T(H, A)$ AND $T(H,-A)=-T(H, A)$

C AFTER T (HABS, AABS) HAS BEEN COMPUTED.

C

$\mathrm{HABS}=\mathrm{ABS}(\mathrm{H})$

$A A B S=A B S(A)$

C

$A 1=A A B S$

$\mathrm{H} 1=\mathrm{HABS}$

C

C

C

C

IF A IS GREATER THAN 1.0, COMPUTE T(AH,1/A) INSTEAD OF $T(H, A)$ AND USE EQUATION RELATING $T(H, A)$ AND $T(A H, 1 / A)$.

IF (AABS. GT.1.0) A1 $=1.0 / \mathrm{A} 1$

IF (AABS.GT.1.0) $\mathrm{H} 1=\mathrm{AABS} * \mathrm{HABS}$

C

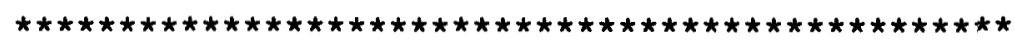

SIMPSON'S RULE NUMERICAL INTEGRATION BEGINS HERE

C

C

$\mathrm{DX}=\mathrm{A} 1 /(\mathrm{REAL}(\mathrm{M})-1.0)$

SUM $=0.0$

$\mathrm{X}=-\mathrm{DX}$

DO $10 I=1, M$

$\mathrm{X}=\mathrm{X}+\mathrm{DX}$

C

C INTEGRAND DEFINED HERE

ARG $=\mathrm{H} 1 * \mathrm{H} 1 *(1.0+\mathrm{X} * \mathrm{X}) / 2.0$

IF (ARG .GT. 50.) ARG $=50.0$

$F=\operatorname{EXP}(-A R G) /(1.0+X \star X)$

$F=F / 6.283185$

C

END OF INTEGRAND DEFINITION

C

C

C

COMPUTE ALTERNATING COEFFICIENTS

IN SIMPSON'S RULE EQUATION

$B=2.0$

$I C=\operatorname{MOD}(I, 2)$

IF ( IC.EQ. O ) $B=4.0$

IF ( I.EQ.1 .OR. I.EQ. M ) $B=1.0$

C

C

ADD IN SUCCESSIVE TERMS HERE

SUM $=S U M+B^{*} F$

C

C

WRITE $(6,2)$ SUM, DX, F, ARG

FORMAT ( 4 (1P E12.3))

C

10

C

C

CONTINUE

NORMALIZE SUM BY DX/3

$T=S U M * D X / 3.0$

C

C END OF SIMPSON'S RULE INTEGRATION

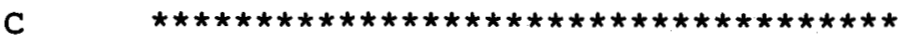

C

C

$\operatorname{WRITE}(6,4) \mathrm{H} 1, \mathrm{~A} 1, \mathrm{~T}$ 
C

C

C

C

C

C

C

C

C

C

C

C

C

C

C

C

C

C

C

C

C

C

C

C

C

C

C

IF ' $A$ ' IS GT 1.0, USE RELATION BETWEEN $T(A H, 1 / A)$ AND $T(H, A)$. WHEN USING THIS EQUATION, ALL ARGUMENTS ARE POSITIVE SINCE WE ARE WORKING WITH ABSOLUTE VALUES OF ARGUMENTS OF T() AT THIS POINT, WITH SIGN OF T() TO BE DETERMINED AT LAST STEP.

IF (AABS. GT. 1.0$) T=($ FN02 (HABS) + FN02 (H1) $) / 2.0$ * -FN02 (HABS) * FN02 (H1) - T

CORRECT SIGN OF T(HABS, AABS) IF A IS NEGATIVE IF(A.IT. 0.0$) T=-T$

WRITE $(6,4) \quad H, A, T$

FORMAT (20X, E12.3)

RETURN

END

FUNCTION FN02 (Z)

THIS FUNCTION COMPUTES THE $N(0,1)$ PROBABILITY GIVEN THE STANDARD NORMAL VARIATE $z$ USING THE INTEGRAL DEFINITION OF THE FUNCTION AND USING TRAPEZOIDAL NUMERICAL INTEGRATION. PROGRAMMED BY MP BOHN, JAN 1989. THIS MORE ACCURATE VERSION IS NEEDED FOR FUNCTION FBNO1.

SHOULD PROBABLY BE IN REAL * 8

$\mathbf{M}=301$

$$
\begin{aligned}
& Z Z=Z \\
& I F(Z . I T .0 .0) \quad z Z=-Z
\end{aligned}
$$

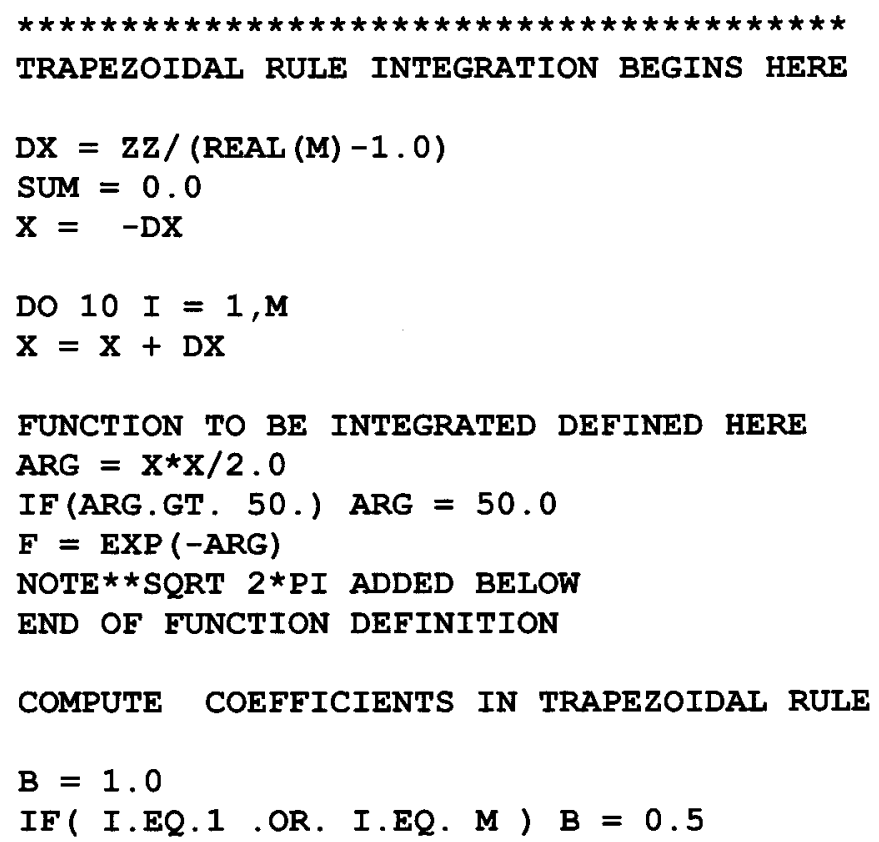




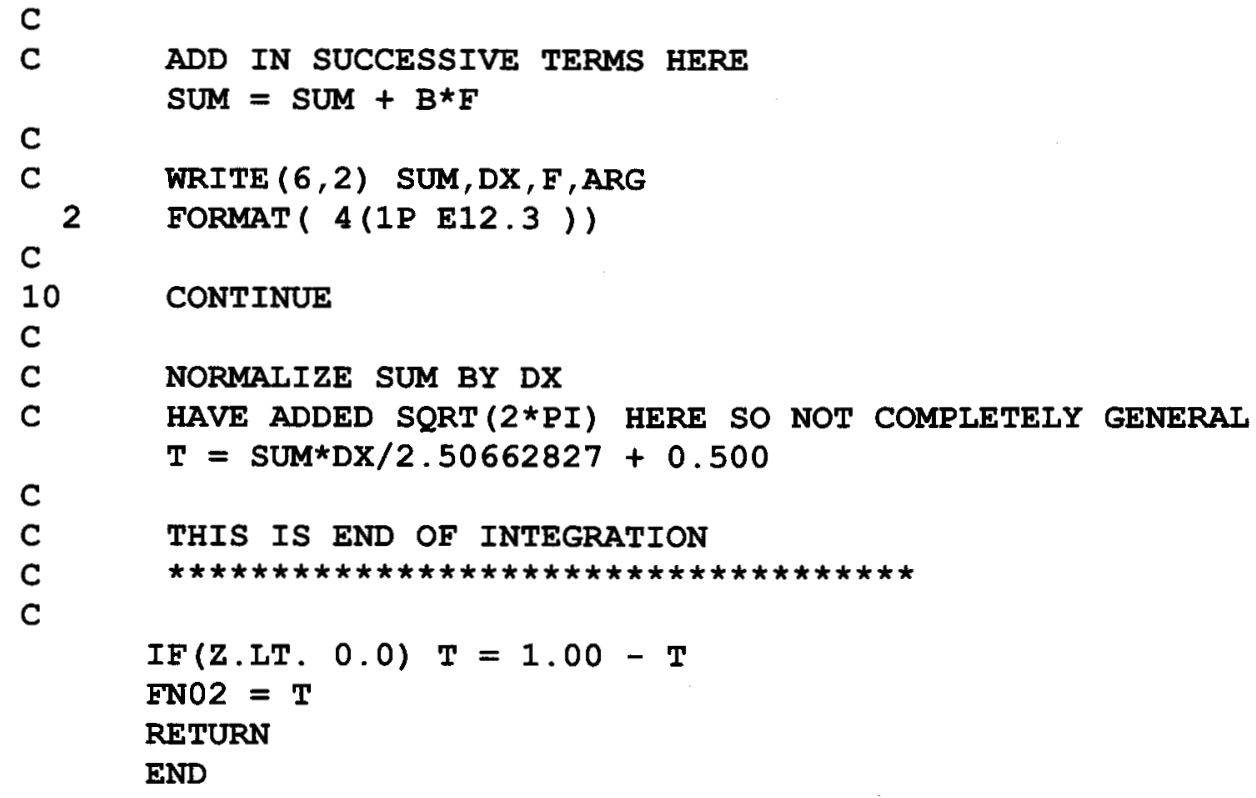




\section{Appendix III: \\ CPLOAS: A FORTRAN Program for the Calculation of the Probability of Loss of Assured Safety}

This appendix contains a description of the FORTRAN program CPLOAS that provides a computational implementation of the procedures described in this report for evaluating the probability of loss of assured safety (i.e., $p F$ in the notation used in the main body of the report). Contents included: (i) Text summary of the algorithms, (ii) Flow charts for the algorithms, (iii) Example input and output for the implementing program, and (iv) source code for the implementing program. 


\section{Overview}

A system of links is defined as a set of links connected in series where each link is defined as either a strong link or a weak link. The system must have at least one strong link and at least one weak link. A strong link is initially represented as an open circuit and a weak link is initially represented as a closed circuit. A failed strong link is represented as a closed (short) circuit and a failed weak link is represented as an open circuit. Each strong link is composed of one or more internal components which are connected in parallel. Strong link internal components have the same circuit definition as a strong link. Failure of any one of the internal components in a strong link results in a failure of that strong link. Failure of the system of links is defined as the failure of all strong links before the failure of any weak link. Each link has an associated temperature failure distribution and an associated timetemperature history. The failure probability of the system of links is determined by the interaction of the link failure distributions and associated link time-temperature histories. The failure temperature for each link is assumed to be independent of the failure temperature for every other link.

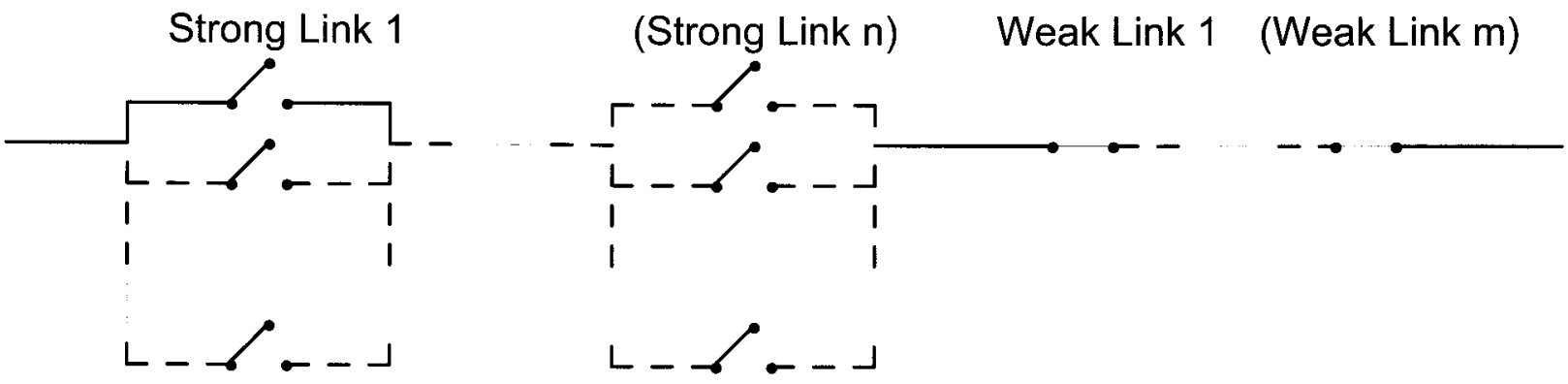

Failure Probability Evaluation for System of Links

Two basic methods are used to estimate the failure probability of the system of links. The first method is based on the integration of a function which defines the interaction of the link failure temperature distributions and associated link temperature histories. The second method is based on Monte Carlo sampling of the link failure temperatures and evaluation of the relative failure times of the system links.

\section{System Failure Function Evaluation}

Two different integration procedures can be used to integrate this system of links function over the temperature range defined by the link temperature histories. Both integration procedures use successive evaluations of the overall system at different temperatures in order to a approximate the overall system failure probability. Initially, the temperature range is a single interval where the failure probability is evaluated at the two endpoints and either a linear (Trapezoid Method) fit or a quadratic (Simpson's Method) fit is assumed for integrating the area between the temperature interval endpoints. The failure probability at the temperature interval midpoint is then evaluated and the area is integrated as the sum of the areas of the two subintervals. This process is repeated using subinterval midpoints until successive failure probability evaluations are within a relative tolerance of each other.

\section{Trapezoid Method for Function Integration}

The Trapezoid Method evaluation begins with averaging evaluations of the system of links failure function at the temperature integration limits. Successive steps in the solution process adjust the previous iteration result by averaging in current iteration failure function evaluations. The next step in the evaluation process is to average in evaluations of the failure function at the midpoint of each temperature subinterval created during the previous iteration. The first iteration is the evaluation of the failure function at the midpoint of the overall temperature interval. The second iteration is the evaluation of the failure function at the two midpoints of the two subintervals created by the first midpoint evaluation. Successive iterations average in failure function evaluations at each of the subinterval 
midpoints. The number of midpoints used for failure function evaluations doubles with each iteration. This iterative process terminates when evaluations for successive iterations differ by less than a predefined relative tolerance.

\section{Simpson's Method for Function Integration}

The Simpson's Method evaluation is identical to the Trapezoid Method evaluation except for the weighting of intermediate evaluations.

\section{Monte Carlo Sampling Evaluation}

Failure temperatures are sampled for each link according to the failure temperature distribution associated with the link. The link failure temperatures are then converted to link failure times through interpolation of the associated time-temperature history for each link. The link failure times are evaluated relative to each other according to the defined system of links to determine whether the system fails for this set of sampled failure temperatures. This process is repeated for a large number of sets of sampled link failure temperatures. The failure probability of the system of links is the total number of failures divided by the total number of sets of sampled failure temperatures.

\section{Importance-Weighted Monte Carlo Sampling Evaluation}

Failure temperatures are sampled for each link according to a predefined sampling distribution assigned to the type of failure temperature distribution associated with the link. A weight is calculated for the link failure temperature based on the evaluation of the probability density function for the failure temperature distribution associated with the link. The sample weight for a set of sampled link failure temperatures is the product of the weights for the individual link failure temperatures. The link failure temperatures are then converted to link failure times through interpolation of the associated time-temperature history for each link. The link failure times are evaluated relative to each other according to the defined system of links to determine whether the system fails for this set of sampled failure temperatures. This process is repeated for a large number of sets of sampled failure temperatures. The failure probability of the system of links is the sum of the weights for sampled sets of link failure temperatures resulting in system failure divided by the total number of sets of sampled link failure temperatures.

\section{Evaluating System of Links for Monte Carlo Sampling and Importance-Weighted Monte Carlo Sampling}

The failure time $\left(\operatorname{tSLn}_{\min }\right)$ for each strong link is evaluated as the minimum failure time of the internal components that comprise the strong link. The failure time $\left(\mathrm{tSL}_{\max }\right)$ of all strong links together is evaluated as the maximum failure time across all strong links. The failure time $\left(\mathrm{tWL}_{\min }\right)$ of all weak links together is evaluated as the minimum failure time across all weak links. If the maximum failure time $\left(\mathrm{tSL}_{\max }\right)$ for all strong links together is less than the minimum failure time ( $\left(\mathrm{WL}_{\min }\right)$ for all weak links together, the overall system of links has failed. 


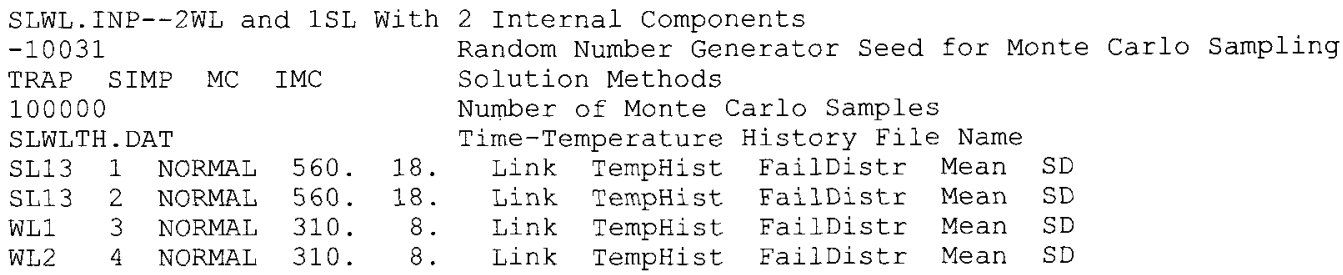

\section{Format Description}

All data read from input file are free (list-directed) format. Comments may be placed after the required input data on any input record.

\begin{tabular}{|c|c|c|}
\hline Record & Type & Description \\
\hline 1 & Character & Title $($ Maximum $=100$ characters $)$ \\
\hline 2 & Integer & Random number generator seed for Monte Carlo sampling \\
\hline 3 & Character & $\begin{array}{l}\text { Solution methods (one or more of following uppercase abbreviations on single } \\
\text { line separated by at least one space) } \\
\text { TRAP--Trapezoid Method used for integration of system failure function } \\
\text { SIMP--Simpson's Method used for integration of system failure function } \\
\text { MC--Monte Carlo sampling method } \\
\text { IMC--Importance-weighted Monte Carlo sampling method }\end{array}$ \\
\hline 4 & Integer & $\begin{array}{l}\text { Number of Monte Carlo samples used for solution methods MC and IMC when } \\
\text { this value is positive. If this value is negative, the system failure probability is } \\
\text { evaluated from time zero through each of the time-temperature history } \\
\text { timesteps using specified integration solution methods (TRAP and/or SIMP). } \\
\text { The Monte Carlo sampling methods (MC and/or IMC) would not be used, even } \\
\text { if specified. }\end{array}$ \\
\hline $\begin{array}{c}5 \\
6+\end{array}$ & Character & $\begin{array}{l}\text { Time-temperature history file name } \\
\text { Link definitions: }\end{array}$ \\
\hline & Character & $\begin{array}{l}\text { Link name ( } 2-10 \text { alphanumeric characters of which first two } \\
\text { characters must be either uppercase SL or uppercase WL. Additional } \\
\text { characters are case-sensitive. Strong links are identified by their internal } \\
\text { components. Each strong link must have a different name. Internal } \\
\text { components within a strong link must have identical names. Weak links have } \\
\text { no naming restrictions other than the first two characters) }\end{array}$ \\
\hline & Integer & Index of link time-temperature history from time-temperature history file \\
\hline & Character & $\begin{array}{l}\text { Failure temperature distribution (one of the following uppercase abbreviations; } \\
\text { failure temperature units must be same as temperature units on } \\
\text { time-temperature history): } \\
\text { UNIFORM = Uniform distribution } \\
\text { NORMAL = Normal distribution } \\
\text { LOGUNIFORM = Loguniform distribution } \\
\text { LOGNORMAL = Lognormal distribution } \\
\text { For uniform distribution: }\end{array}$ \\
\hline & Real & Minimum value for uniform distribution \\
\hline & Real & $\begin{array}{l}\text { Maximum value for uniform distribution } \\
\text { For normal distribution: }\end{array}$ \\
\hline & Real & Mean value for normal distribution \\
\hline & Real & $\begin{array}{l}\text { Standard deviation (sigma) for normal distribution } \\
\text { For loguniform distribution: }\end{array}$ \\
\hline & Real & Minimum value for loguniform distribution \\
\hline
\end{tabular}




$\begin{array}{ll}\text { Real } & \text { Maximum value for loguniform distribution } \\ & \text { For lognormal distribution: }\end{array}$

\section{$\underline{\text { SL/WL Time-Temperature History File Description }}$}

$\begin{array}{crrrr}\text { Time Temperature Histories for } & \text { 2WL and } 1 \text { SI with Internal Components } \\ 0.0 & 61.53 & 64.67 & 44.98 & 42.77 \\ 10.0 & 413.13 & 439.46 & 264.81 & 226.94 \\ 20.0 & 701.01 & 733.40 & 517.81 & 402.38 \\ 30.0 & 867.29 & 891.94 & 697.01 & 560.15 \\ 40.0 & 950.48 & 965.56 & 819.31 & 687.31 \\ 50.0 & 989.11 & 997.37 & 898.84 & 784.66 \\ 60.0 & 1006.43 & 1010.68 & 948.14 & 856.32 \\ 70.0 & 1014.06 & 1016.18 & 977.83 & 907.55 \\ 80.0 & 1017.41 & 1018.44 & 995.41 & 943.42 \\ 90.0 & 1018.87 & 1019.36 & 1005.71 & 968.16 \\ 100.0 & 1019.51 & 1019.74 & 1011.72 & 985.05\end{array}$

\section{Format Description}

All data read from input file are free (list-directed) format. Comments may be placed after the required input data on any input record.

$\begin{array}{cll}\begin{array}{c}\text { Record } \\ 1\end{array} & \text { Type } & \text { Description } \\ 2+ & & \begin{array}{l}\text { Title (Maximum }=100 \text { characters) } \\ \text { Link temperatures versus time (each record corresponds to one time with } \\ \text { temperatures for each link at that time; times must be monotonically increasing } \\ \text { from record to record): }\end{array} \\ & \text { Real } & \begin{array}{l}\text { Time } \\ \text { Real }\end{array} \\ & \begin{array}{l}\text { Temperature for each link at this time (one or more separated by at least one } \\ \text { space) }\end{array}\end{array}$




\section{Overall Calculation Structure}

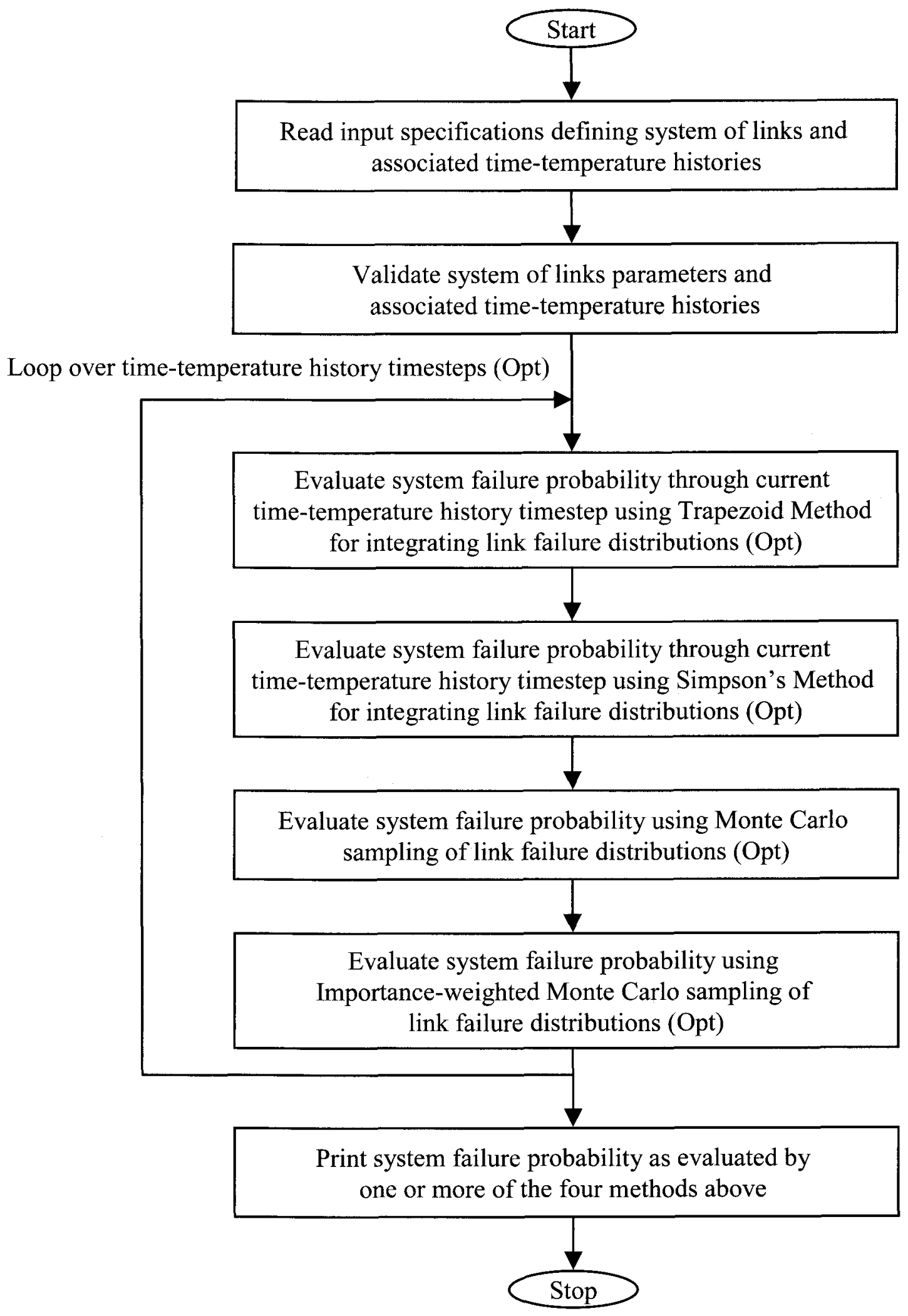




\section{System Failure Function Structure}

Interpolate failure time for current strong link internal component using associated time-temperature history at current temperature

Loop over strong link internal components

\begin{tabular}{|c|}
\hline $\begin{array}{c}\text { Evaluate probability density function for current strong link } \\
\text { internal component at current temperature }\end{array}$ \\
$\begin{array}{c}\text { Evaluate probability that each of the other strong link } \\
\text { internal components for current strong link has not failed at } \\
\text { current strong link internal component failure time }\end{array}$ \\
$\begin{array}{c}\text { Evaluate probability that at least one internal component of } \\
\text { every other strong link has failed at current strong link internal } \\
\text { component failure time }\end{array}$ \\
\hline $\begin{array}{c}\text { Evaluate probability that each of the weak links has not failed at } \\
\text { current strong link internal component failure time }\end{array}$ \\
\hline $\begin{array}{c}\text { Evaluate contribution of current strong link internal component to } \\
\text { system failure probability at current temperature as the } \\
\text { product of the previous four probabilities }\end{array}$ \\
the probability products over all strong link internal components \\
at current temperature
\end{tabular}




\section{Trapezoid Method Integration Structure}

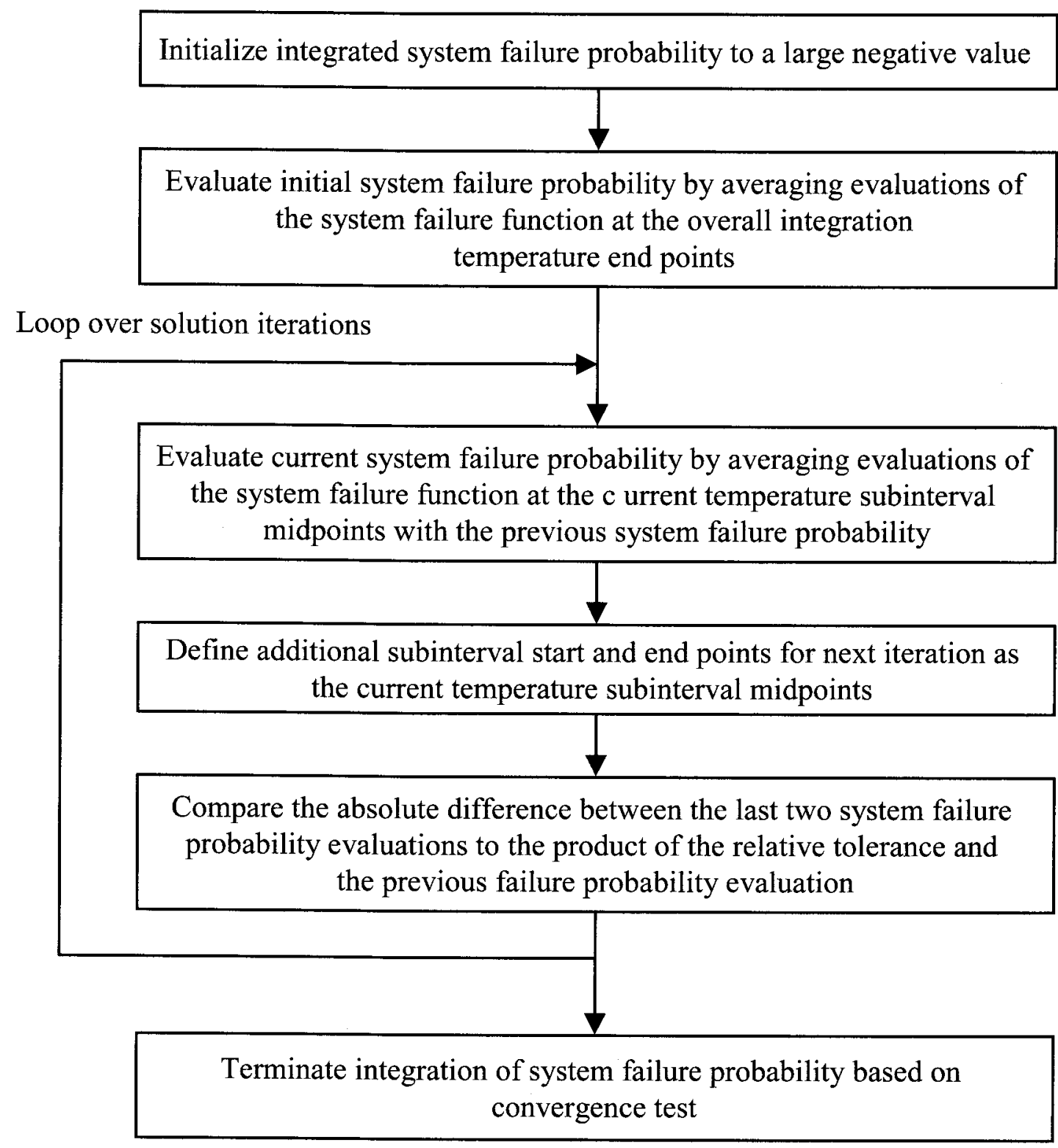




\section{Simpson's Method Integration Structure}

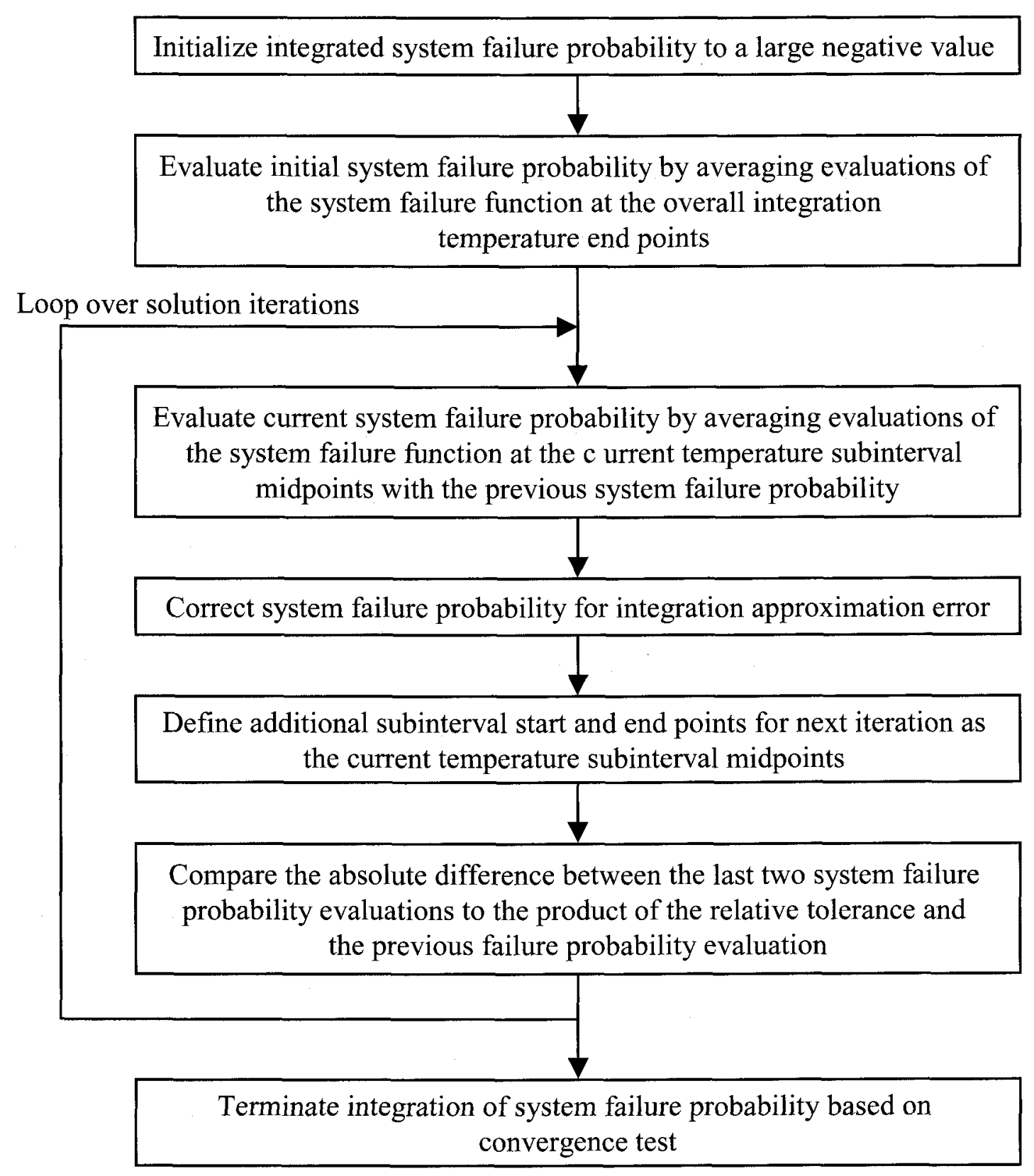




\section{Monte Carlo Sampling Structure}

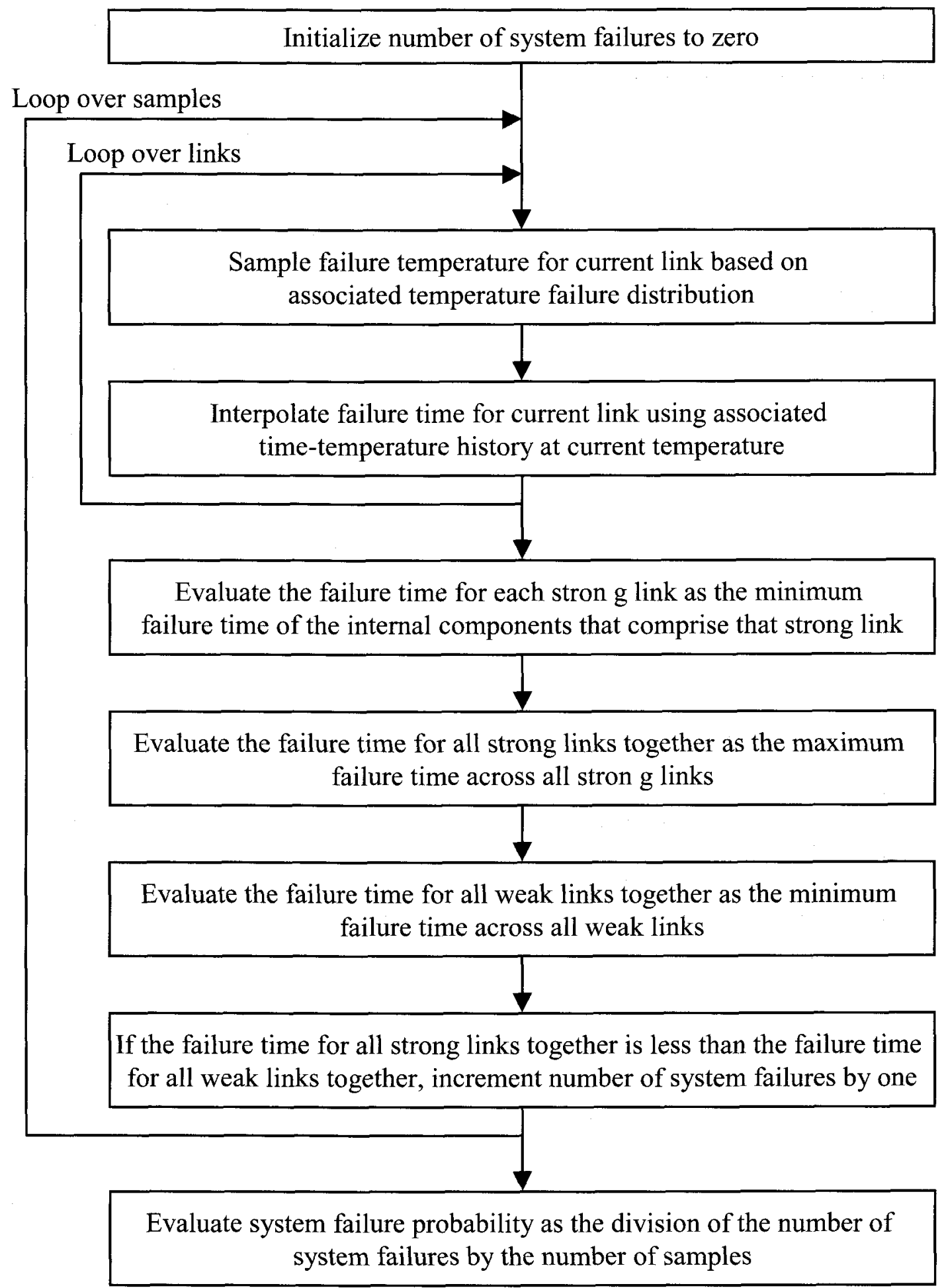




\section{Importance-Weighted Monte Carlo Sampling Structure}

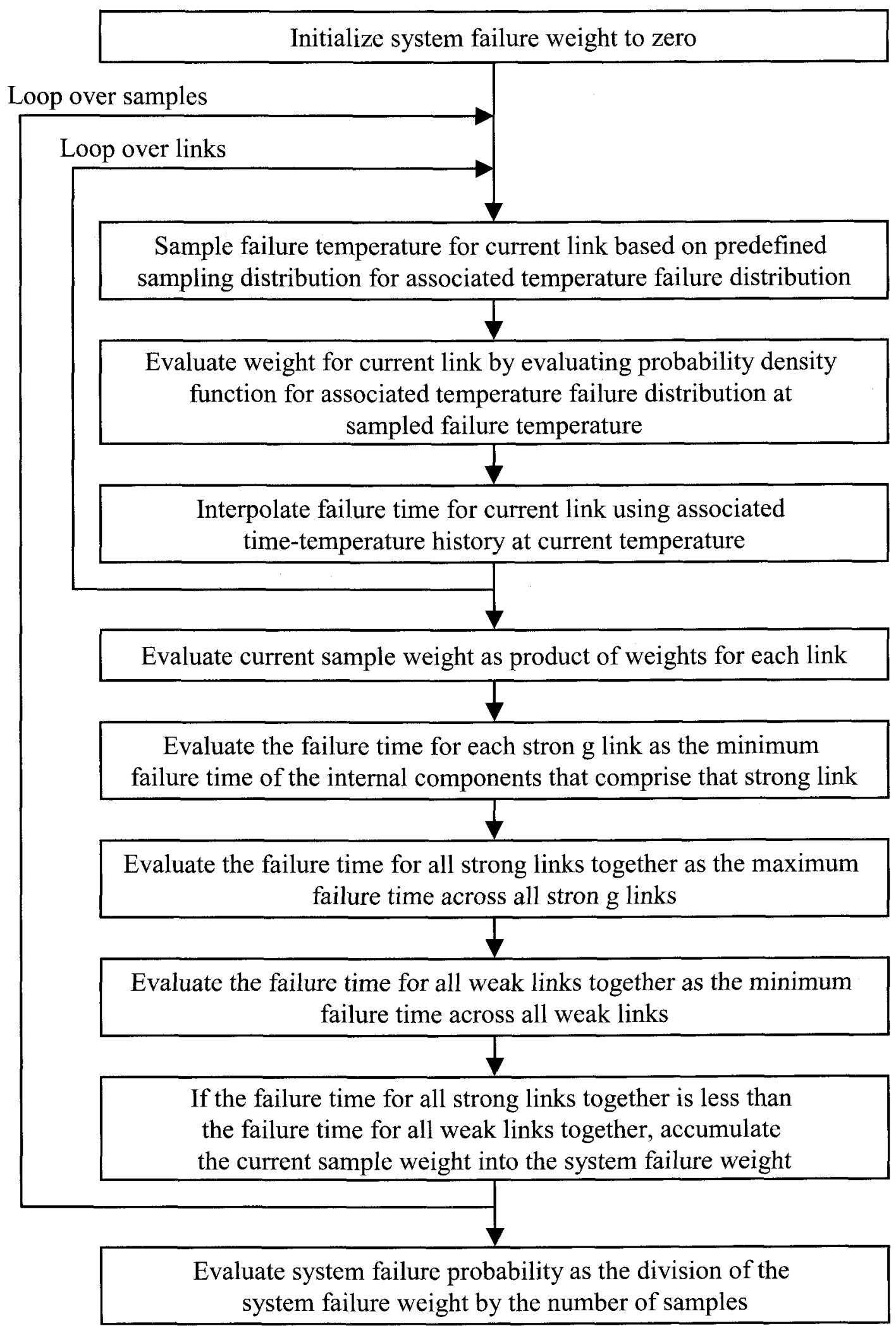


Example Input File for Strong Link / Weak Link Failure Probability Calculation

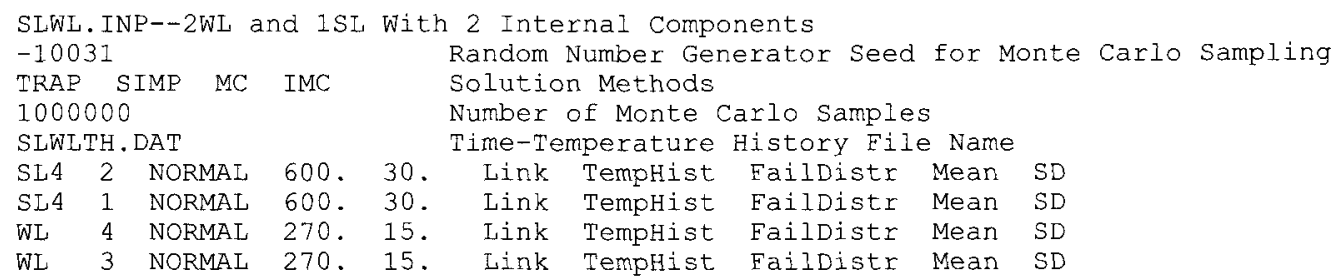

Example Time-Temperature History File for Strong Link / Weak Link Failure Probability Calculation

$\begin{array}{crrrrr}\text { Time Temperature Histories for } & \text { 2WL and } 1 S L \text { with Internal Components } \\ 0.0 & 61.53 & 64.67 & 44.98 & 42.77 \\ 10.0 & 413.13 & 439.46 & 264.81 & 226.94 \\ 20.0 & 701.01 & 733.40 & 517.81 & 402.38 \\ 30.0 & 867.29 & 891.94 & 697.01 & 560.15 \\ 40.0 & 950.48 & 965.56 & 819.31 & 687.31 \\ 50.0 & 989.11 & 997.37 & 898.84 & 784.66 \\ 60.0 & 1006.43 & 1010.68 & 948.14 & 856.32 \\ 70.0 & 1014.05 & 1016.18 & 977.83 & 907.55 \\ 80.0 & 1017.41 & 1018.44 & 995.41 & 943.42 \\ 90.0 & 1018.87 & 1019.36 & 1005.71 & 968.16 \\ 100.0 & 1019.51 & 1019.74 & 1011.72 & 985.05\end{array}$

Example Output File for Strong Link / Weak Link Failure Probability Calculation

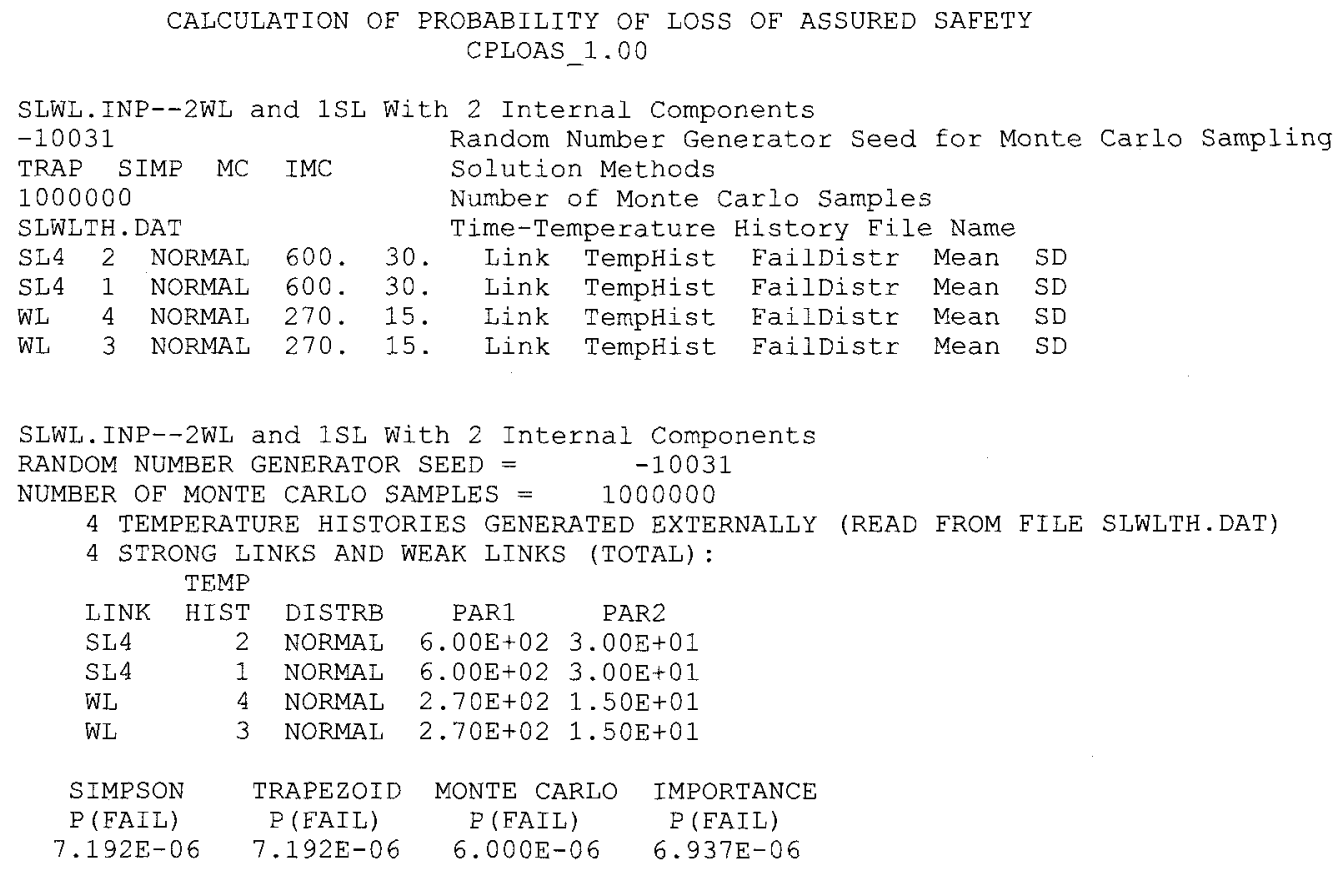


PROGRAM CPLOAS

$C * \star \star \star *$ CALCULATION OF PROBABILITY OF LOSS OF ASSURED SAFETY

$C * * \star * \star$ CALCULATE FAILURE PROBABILITY FOR SYSTEM COMPOSED OF STRONG LINKS

$C * * \star * *$ AND WEAK LINKS HAVING SPECIFIED FAILURE DISTRIBUTION PARAMETERS AND C*****ASSOCIATED THERMAL HISTORIES

$C^{\star \star *} \star \star *(1)$ SERIES FAULTS--FAILURE OF ALI STRONG LINKS BEFORE ANY

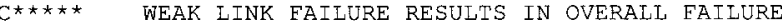

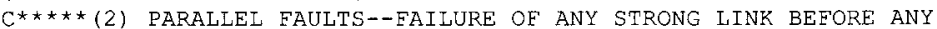

$C^{\star * \star * \star *}$ WEAK IINK FAILURE RESULTS IN OVERALL FAILURE

$C^{*} \star * * \star(3)$ SERIES FAULTS WITH INTERNAL COMPONENTS--FAILURE OF ALL

$\mathrm{C}^{\star \star \star \star \star *}$ STRONG LINKS BEFORE ANY WEAK IINK FAILURE RESULTS IN

C***** OVERALI FAILURE. EACH STRONG LINK CAN BE COMPOSED OF ONE OR

$C \star \star \star \star \star$ MORE INTERNAL COMPONENTS WHERE ANY INTERNAL COMPONENT FAILURE

C***** RESULTS IN FAILURE OF THAT STRONG LINK

IMPLICIT DOUBLE PRECISION (A-H, O-Z)

PARAMETER (MAXTIM $=1000, \operatorname{MAXLNK}=100, \operatorname{MAXDST}=4, \operatorname{MAXEDP}=10)$

CHARACTER $* 10 \mathrm{CL}, \mathrm{CFD}$

CHARACTER $* 10$ VSN

CHARACTER * 100 TITLE, FILINP, FILOUT, THFILE

LOGICAL TRAP, SIMP, MC, IMC

COMMON /FAIL0/ TITLE, FILINP, FILOUT, THFILE, VSN, CL (MAXLNK) 1 CFD (MAXLNK)

COMMON /EAIL1/ ISEED, ILF, NLINK, NTH, NTIME, N, ITHL (MAXIK) , ITFD (MAXINK) NPFD (MAXDST)

COMMON /FAIL2/ DUMMY, PEAILM, PEAILI, PFAILS,

1 PFAILT, THTIME, TMIN, TMAX,

$\mathrm{T}(0:$ MAXTIM)

TH (0:MAXTIM, MAXLNK),

THMAX (O:MAXTIM, MAXLNK),

TFD (MAXFDP, MAXLNK).

$\begin{array}{ll}6 & \text { TET (MAXEDP, MAXLNK) } \\ \text { COMMON /FAIL3/ TRAP, SIMP, MC, IMC }\end{array}$

CHARACTER $* 200 \mathrm{REC1}, \mathrm{REC} 2$

DIMENSION PETMP (4)

C

$\mathrm{C} * \star * * *$ SET VERSION NUMBER

$\mathrm{VSN}=11.001$

$C \star \star \star \star \star$ REQUEST INPUT FILE NAME

WRITE $(6, *)$ ' ENTER INPUT FILE NAME--'

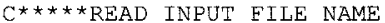

$\operatorname{READ}(5,1001)$ FILINP

IC $=$ INDEX (FILINP, ' $'$ )

IF (IC . GT . 0) THEN

ELSE

FILOUT $=$ FILINP $(1:$ IC $) / /$ 'OUT'

IC=INDEX (FILINP, ‘ ’)

ENDIE

WRITE $(6,1101)$ FILOUT (1: INDEX (EILOUT,' ')-1)

$\mathrm{C} \star \star \star \star *$ OPEN OUTPUT FILE

OPEN $(2$, FILE=EILOUT, STATUS='UNKNOWN' $)$

WRITE $(2,2001)$ VSN

$C \star \star \star \star \star$ READ INPUT PARAMETERS

CALL READI

$C * * * * *$ VALIDATE INPUT PARAMETERS

CALL VALIDI

$C * * * * \star$ INITIALIZE PARAMETERS

CALL INIT

$C \star \star \star \star \star$ READ LINK THERMAL HISTORIES

CALL RTH

$\mathrm{C}^{*} * * *$ SET STARTING TIMESTEP INDEX

NTIM=NTIME

IF (N.GE. O) THEN

ELSE

TTIMS=NTIME

ENDIF

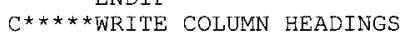

$\mathrm{REC} 1=$ '

$\mathrm{REC} 2=$ '

$\mathrm{NPF}=1$

IF (N.LT. O) THEN

$\mathrm{NC}=10$

$\operatorname{REC} 2(6: 9)=$ 'T TME'

ELSE

$\mathrm{NC}=1$

ENDIF

IF (TRAP) THEN

$\mathrm{NPF}=\mathrm{NPE}+1$

REC1 $(\mathrm{NC}+5: \mathrm{NC}+13)=$ 'TRAPEZOID

$\operatorname{REC} 2(\mathrm{NC}+6: \mathrm{NC}+12)=$ PLOAS ।

$\mathrm{NC}=\mathrm{NC}+12$

ENDIF

IF (SIMP) THEN

$\mathrm{NPF}=\mathrm{NPF}+1$ 


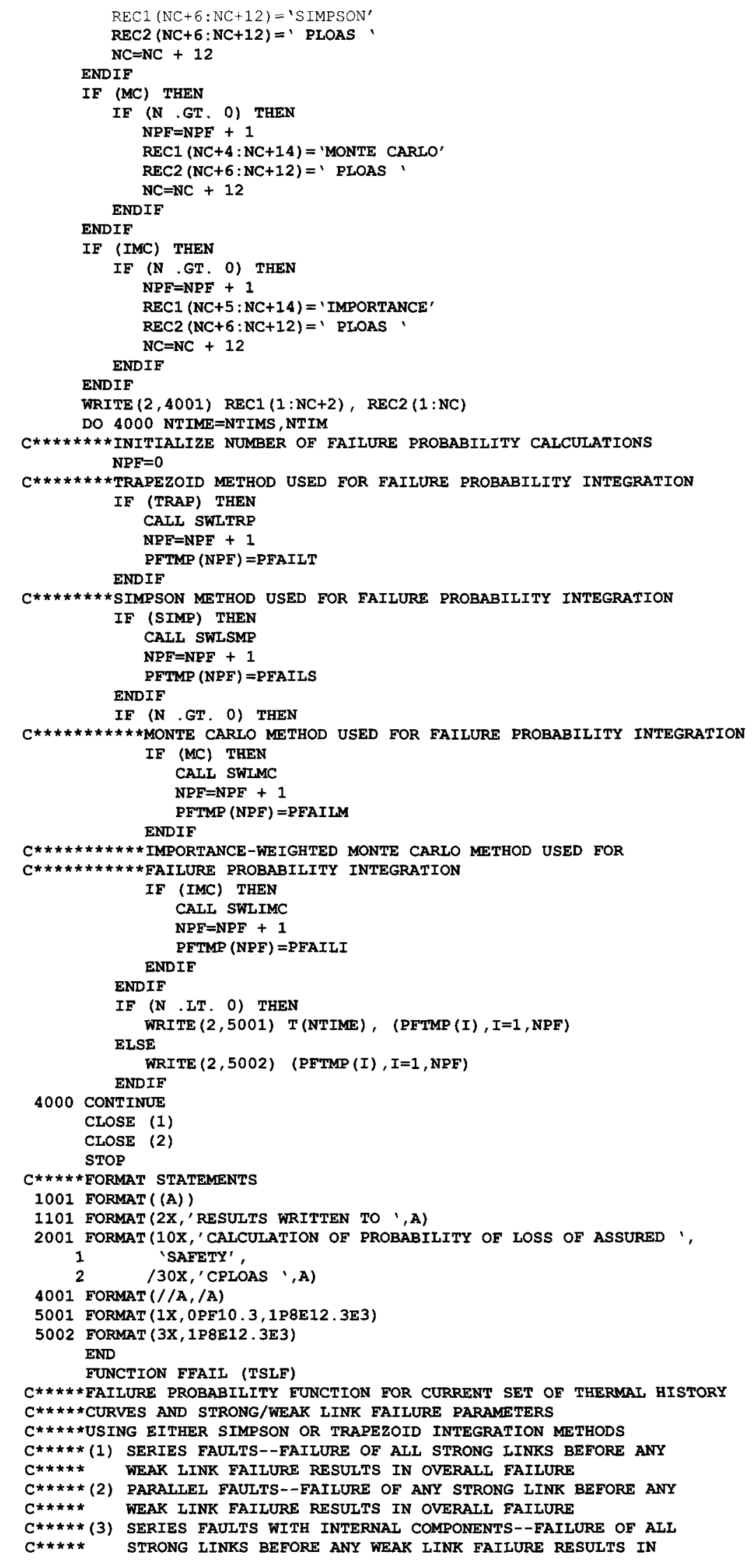




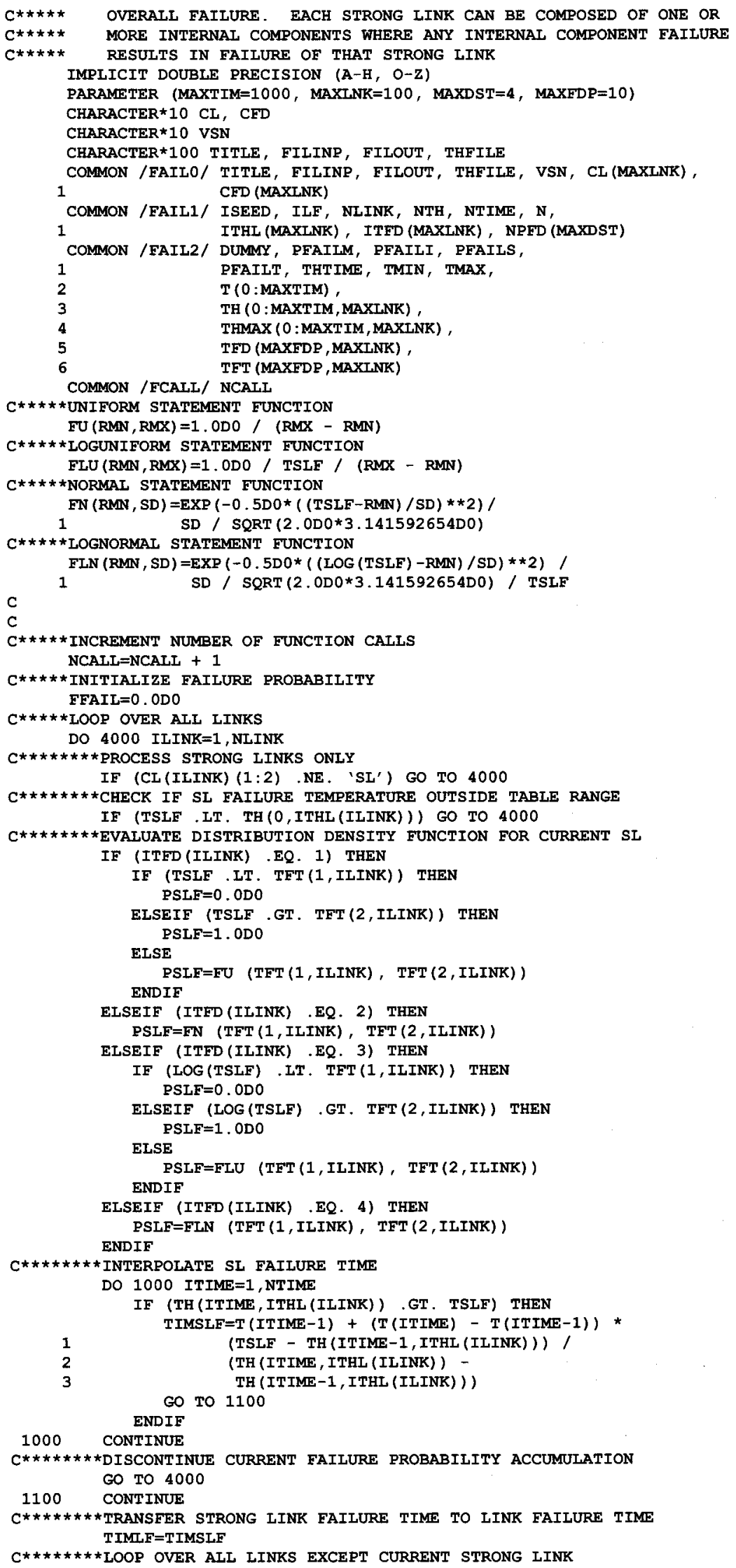




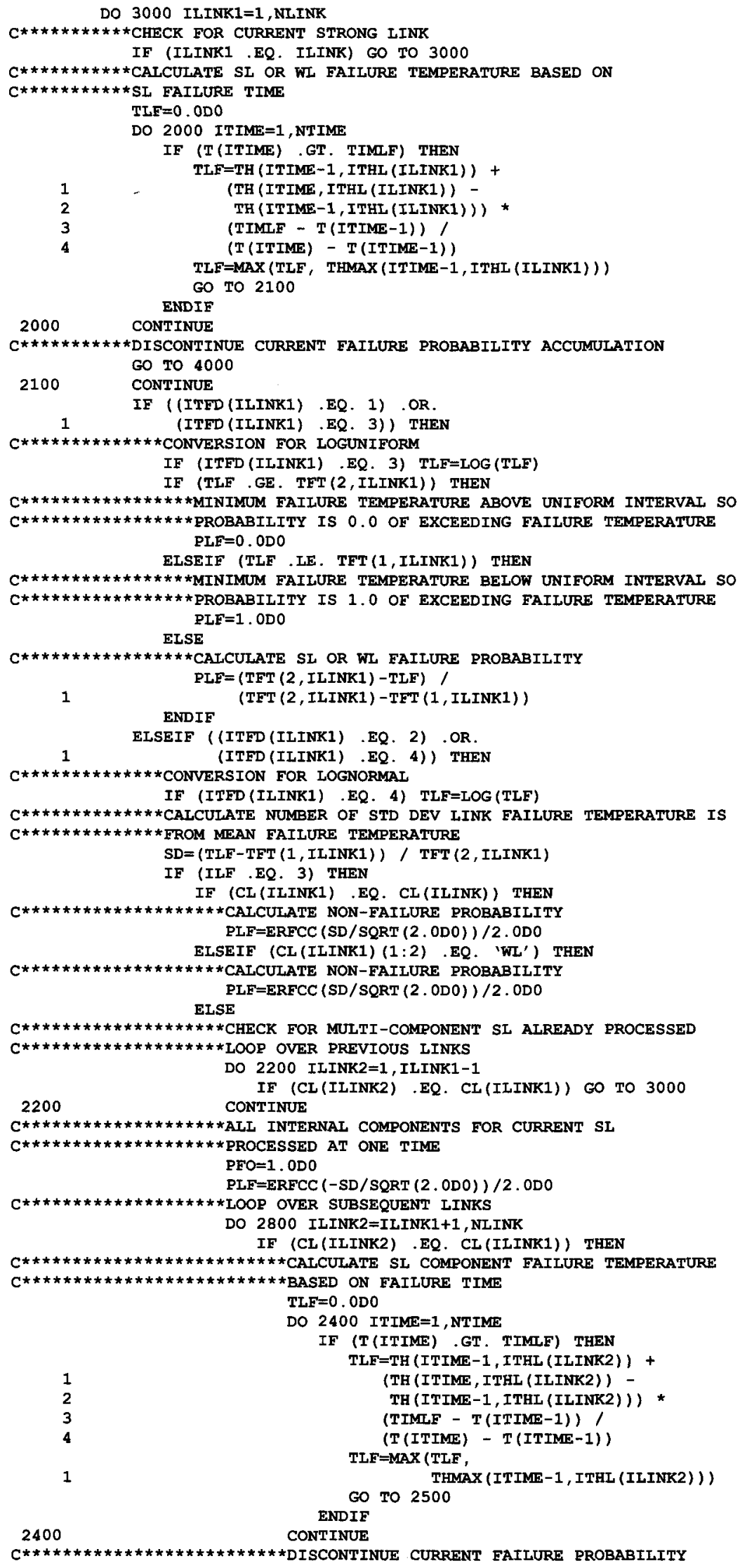$$
\text { ENDIF }
$$

CONTINUT

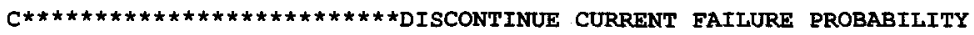




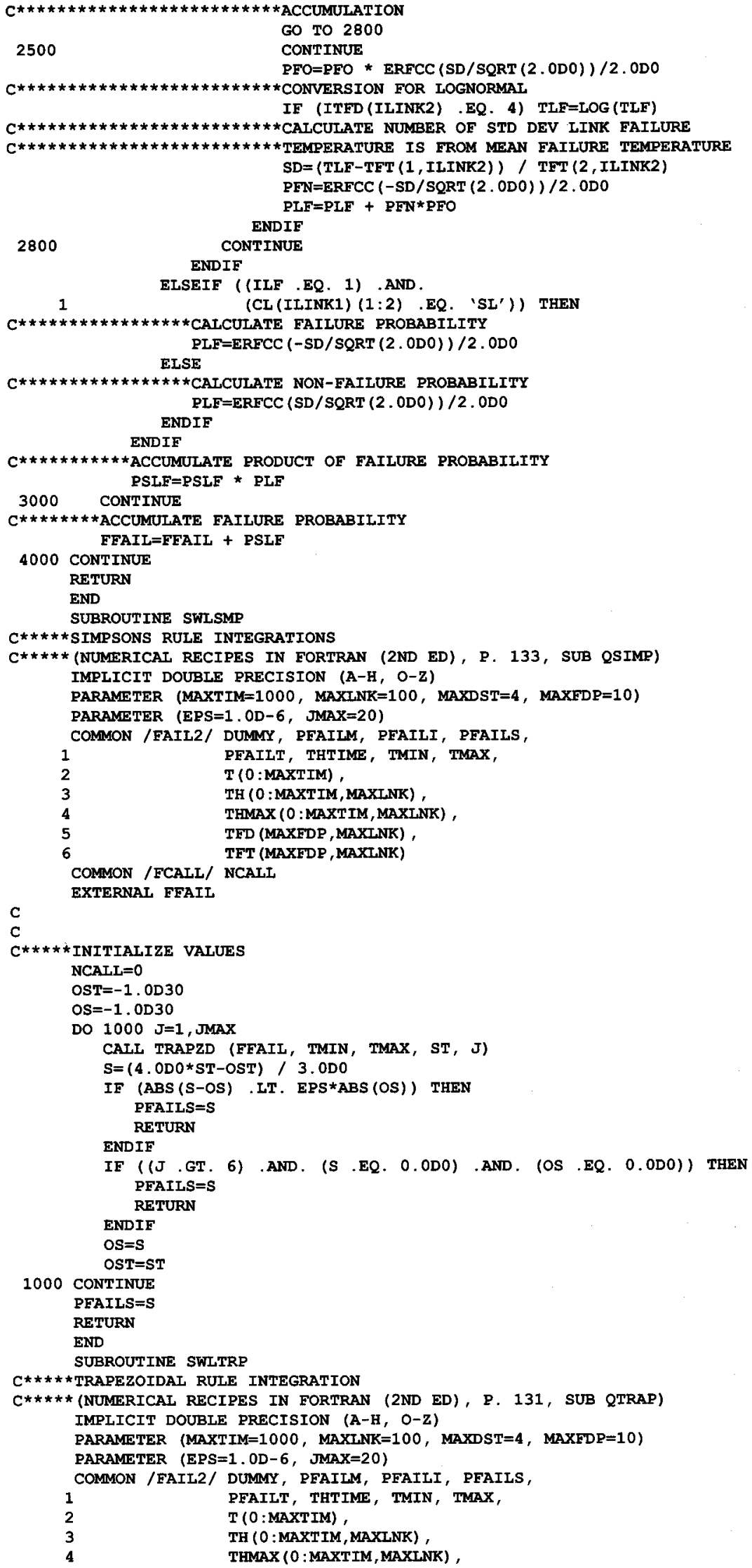




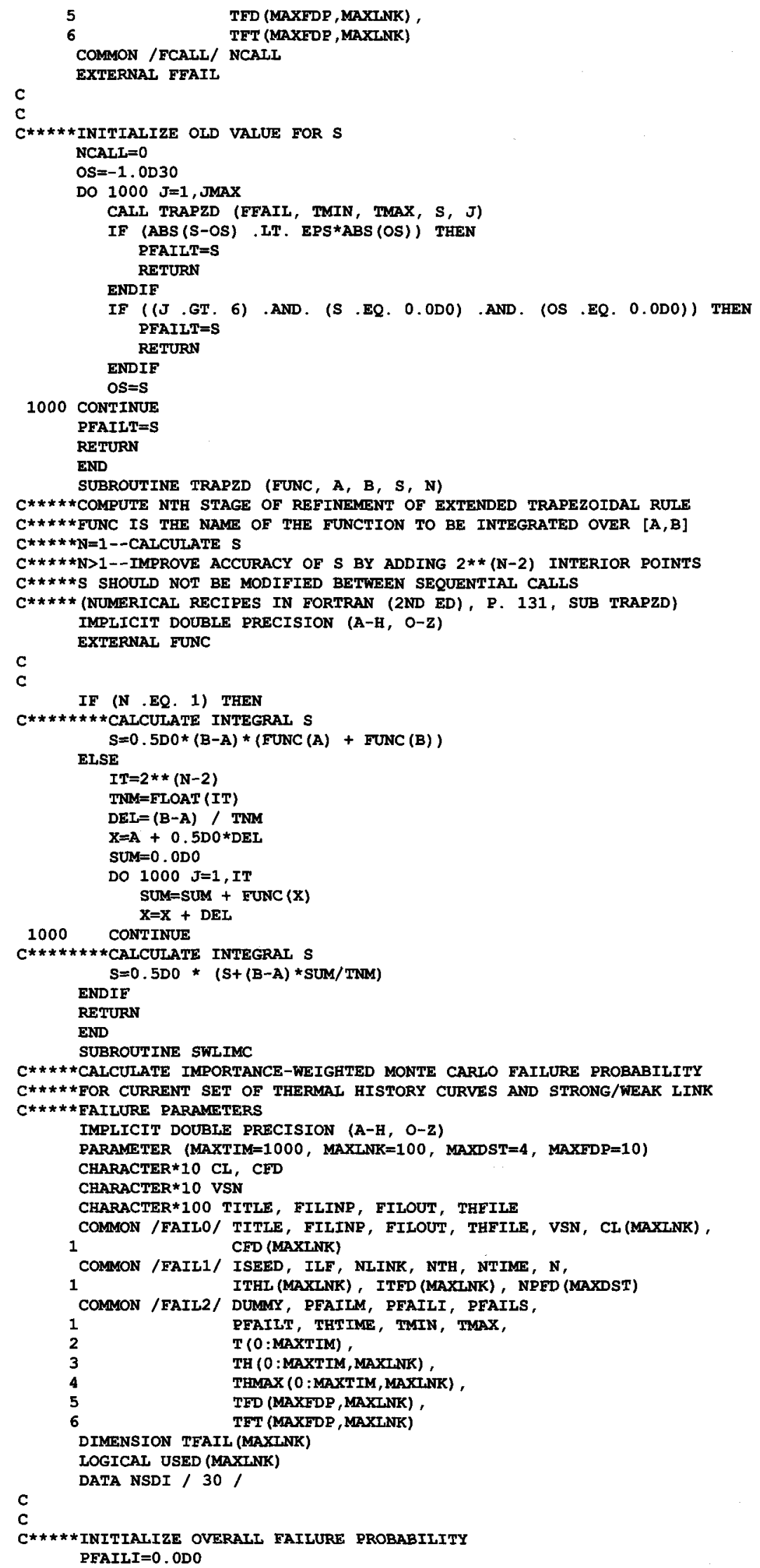




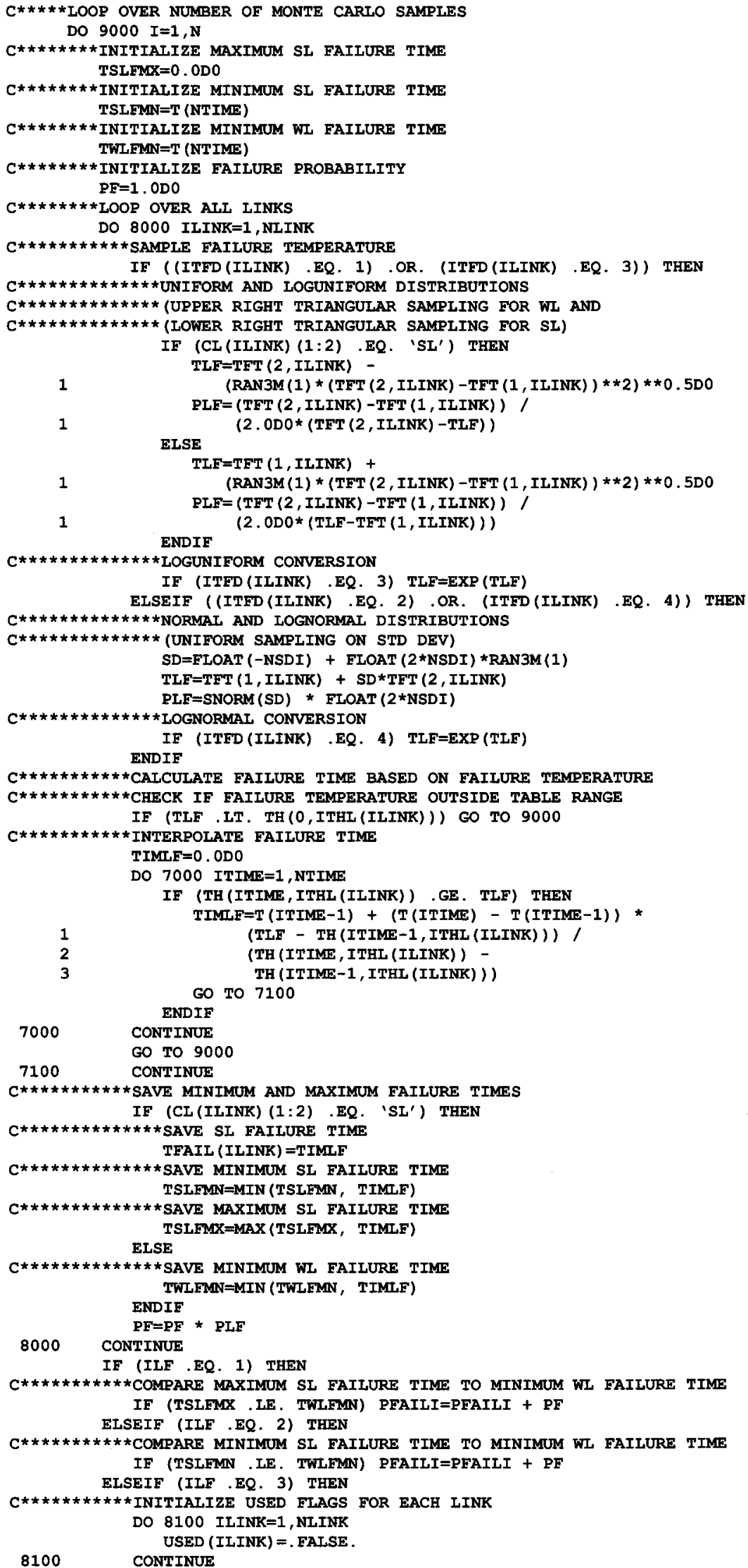

$8100 \quad$ CONTINUE 


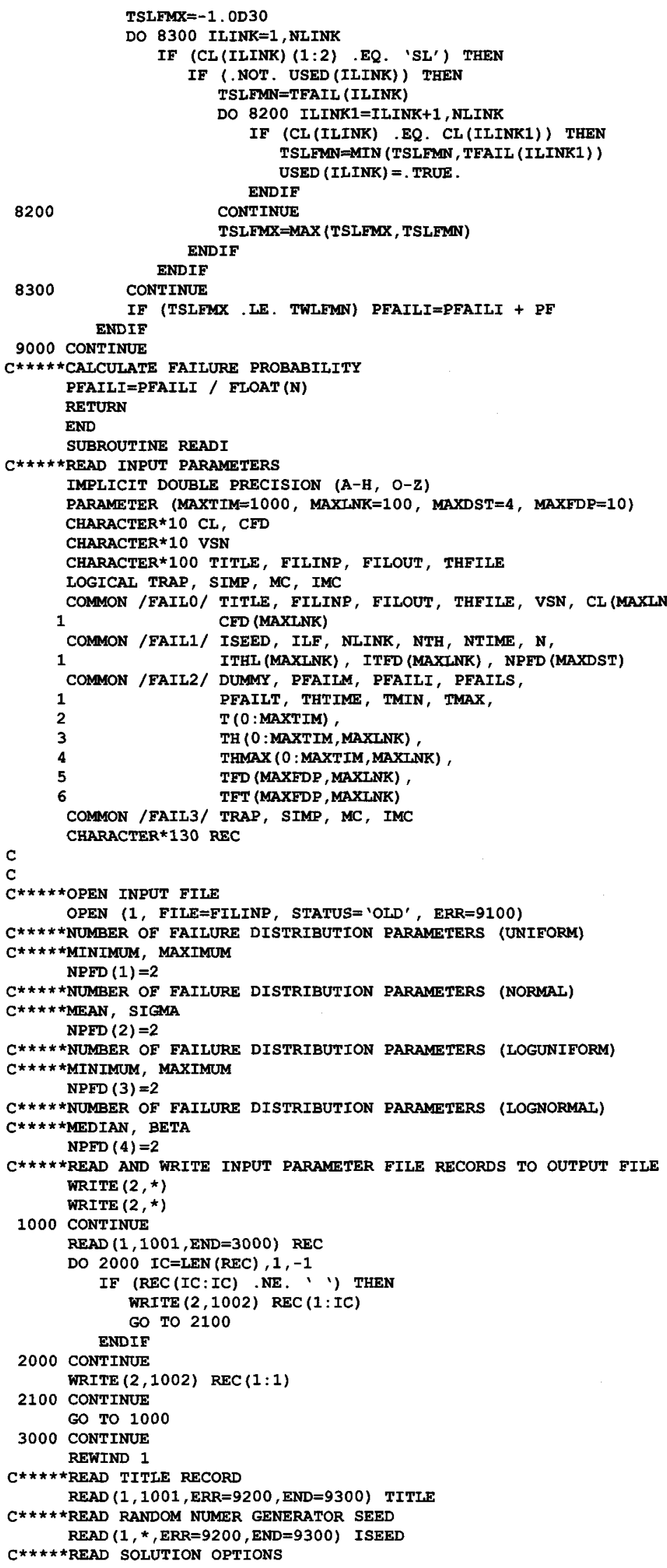




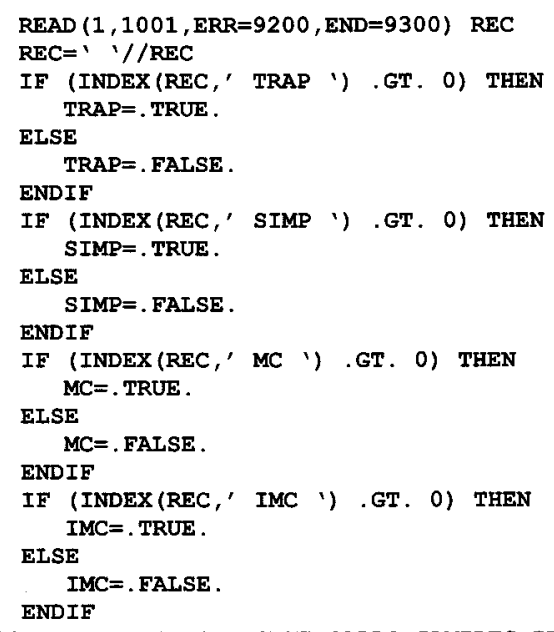




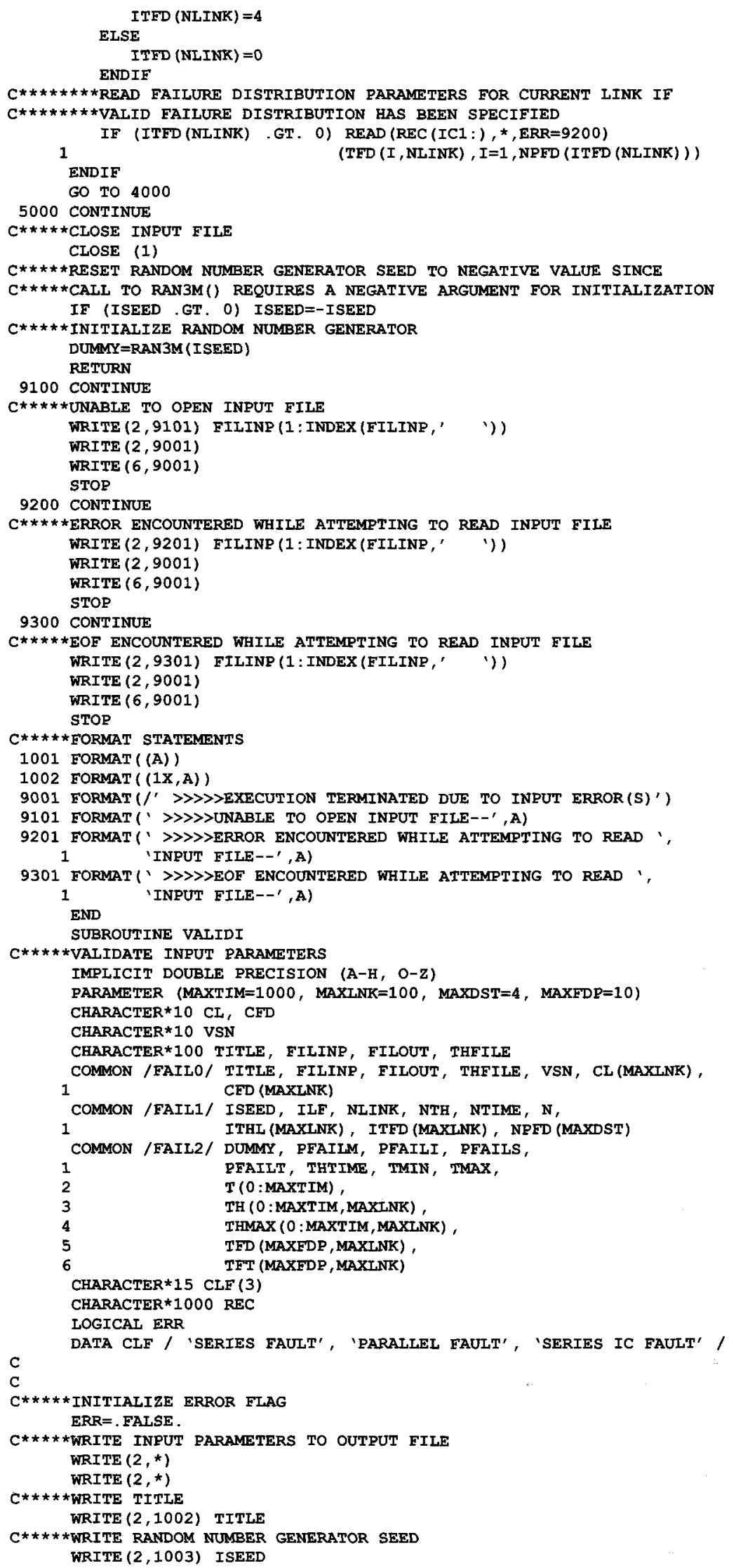




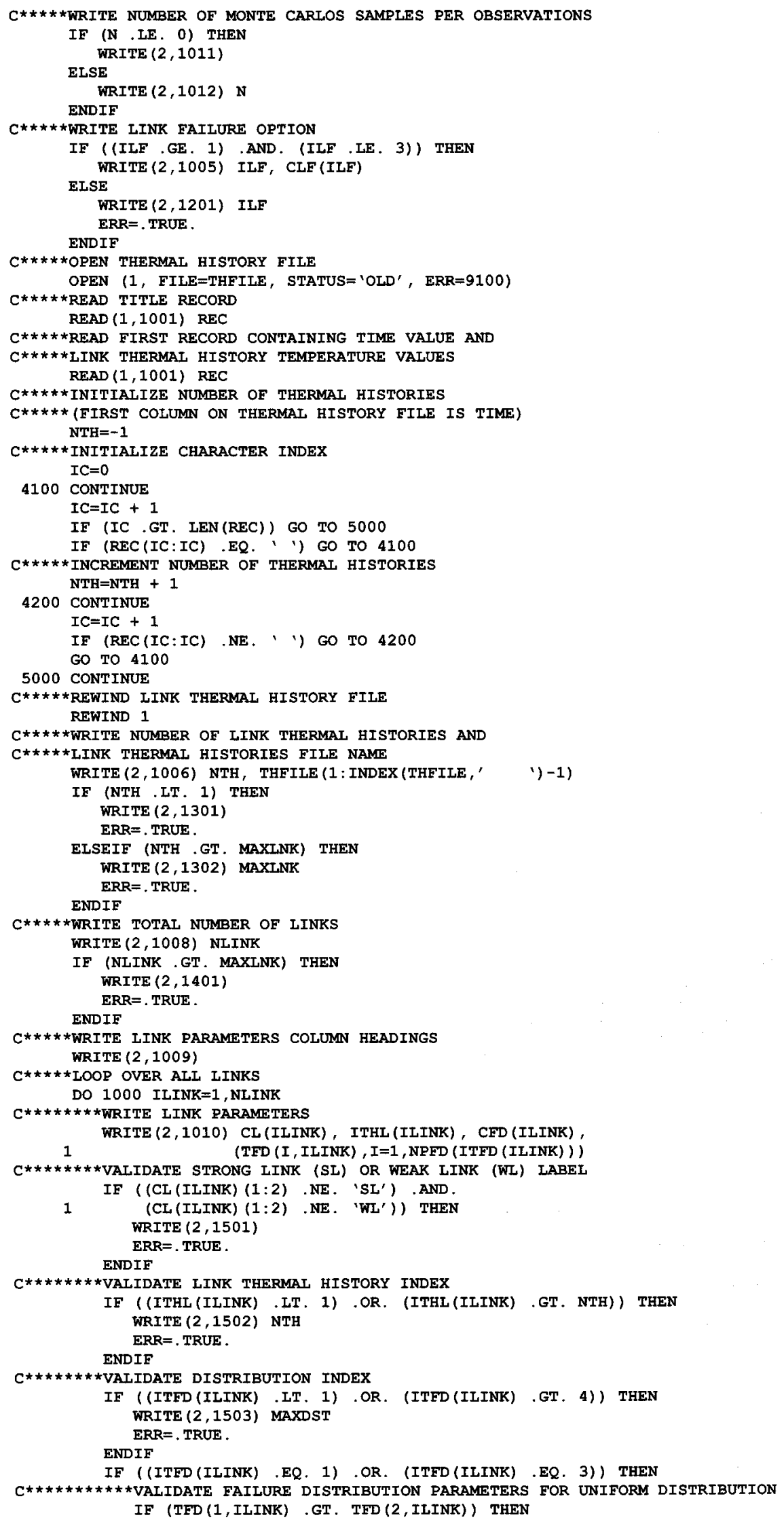




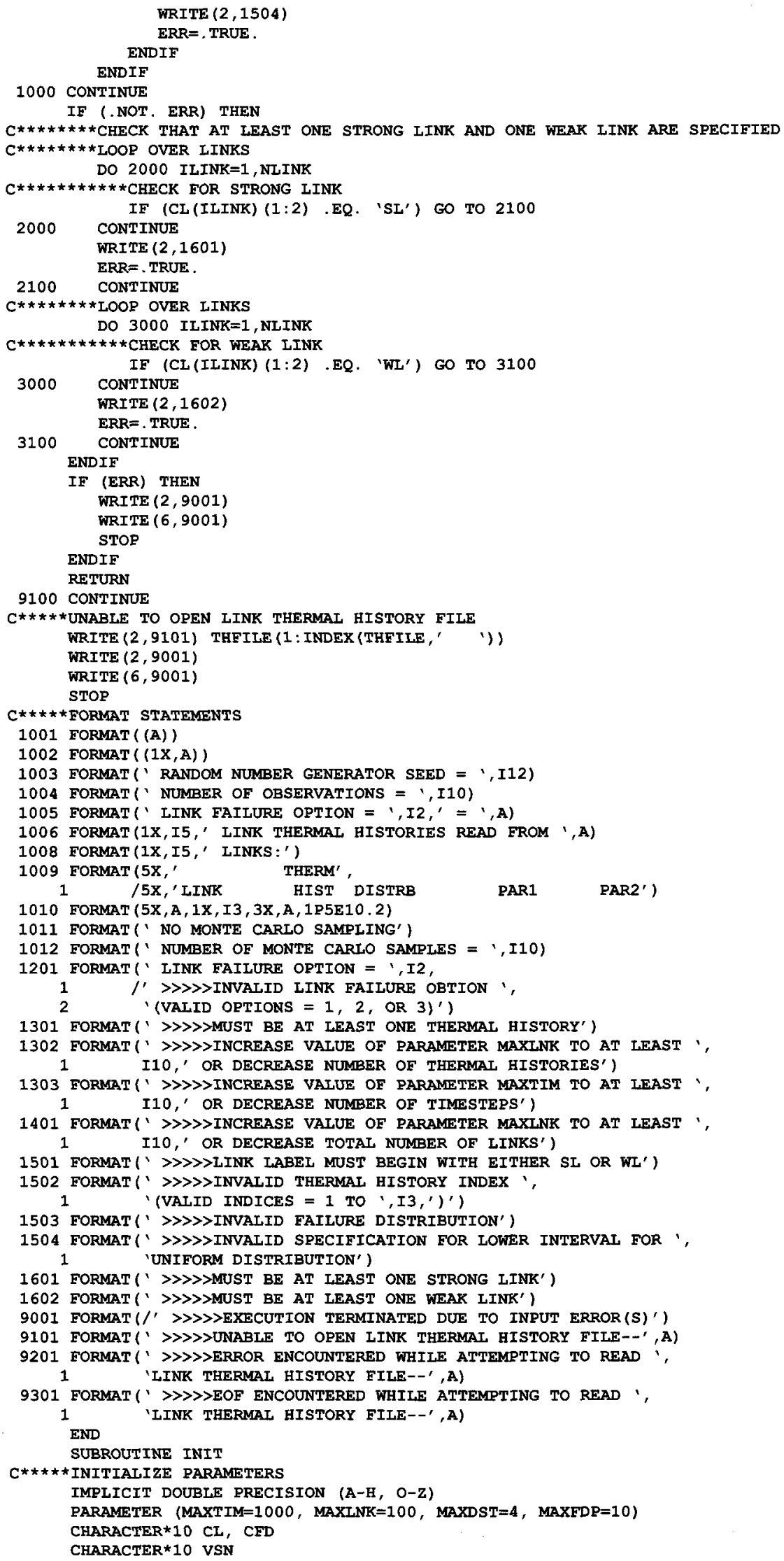




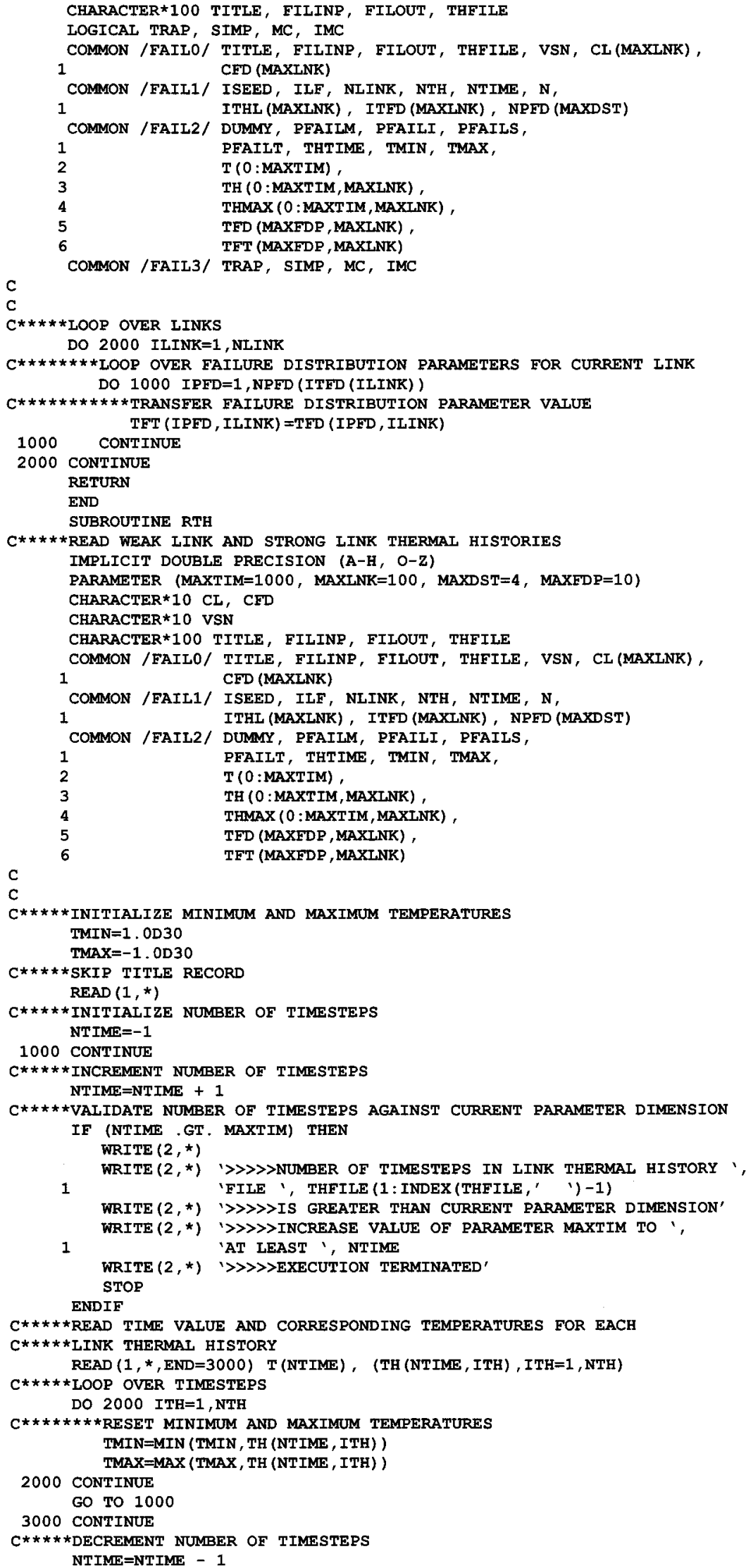


CLOSE (1)

TMIN=MAX (TMIN, $1.0 \mathrm{D}-30$ )

$C \star \star \star \star \star$ SET MAXIMUM LINK TEMPERATURE ENCOUNTERED THROUGH EACH TIME INTERVAI

DO $5000 \mathrm{ITH}=1$, NTH

THMAX $(0, \mathrm{ITH})=\mathrm{TH}(0, \mathrm{ITH})$

DO 4000 ITIME $=1$, NTIME

4000 CONTINUE

THMAX ( ITIME, ITH) =MAX (THMAX (ITIME-1, ITH) , TH (ITIME , ITH) )

5000 CONTINUE

RETURN

END

SUBROUTINE SWLMC

$C \star \star * \star \star$ CALCUILATE MONTE CARLO FAILURE PROBABILITY FOR CURRENT SET OF LINK

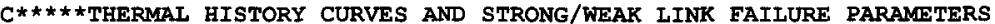

IMPLICIT DOUBLE PRECISION (A-H, O-Z)

PARAMETER (MAXIIM=1000, MAXINK=100, MAXDST=4, MAXFDP=10)

CHARACTER $* 10 \mathrm{CL}$, CFD

CHARACTER $* 10$ VSN

CHARACTER $\star 100$ TITLE, FILINP, FILOUT, THFILE

COMMON /FAIL1/ ISEED, ILF, NLINK, NTH, NTIME, N,

COMMON /FAIL2/ DUMMY, PFAIIM, PFAILI, PFAILS,

1 PFAILT, THTIME, TMIN, TMAX,

$\begin{array}{ll}2 & \text { T }(0: \text { MAXTIM), } \\ 3 & \text { TH }(0: \text { MAXTIM, MAXLNK), }\end{array}$

4 THMAX (0:MAXTIM, MAXINK),

TED (MAXFDP, MAXLNK)

DIMENSION TFAIL (MAXINK)

LOGICAL USED (MAXLNK)

C

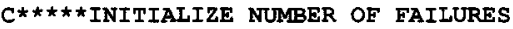

$N F A I L=0$

$C * * \star * *$ LOOP OVER NUMBER OF MONTE CARLO SAMPLES

DO $9000 \quad I=1, N$

$C \star \star \star \star \star \star \star \star$ INITIALIZE MAXIMUM SL FAILURE TIME TSLEMX $=0$. ODO

$C \star * * \star * \star \star *$ INITIALIZE MINIMUM SL FAILURE TIME TSLFMN $=T$ (NTIME)

C $\star \star \star \star \star \star \star \star$ INITIALIZE MINIMUM WL FAILURE TIME TWLEMN $=$ T (NTIME)

$C \star \star \star \star \star \star \star \star$ LOOP OVER ALL LINKS DO 8000 ILINK $=1$, NLINK

C $\star \star \star \star \star \star \star \star \star \star \star \star$ SAMPLE FAILURE TEMPERATURE

IF ((ITFD (ILINK) .EQ. 1) .OR. (ITFD (ILINK) .EQ. 3)) THEN

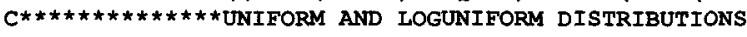
TLF $=$ TFT $(1$, ILINK $)+($ TFT $(2, \operatorname{ILINK})-T F T(1, \operatorname{ILINK})) *$ RAN3M $(1)$

C $* * \star \star \star \star \star \star \star * \star * * * \star *$ LOGUNIFORM CONVERSION IF (ITFD (ILINK) .EQ. 3) TLF=EXP (TLF) ELSEIF ((ITFD (ILINK) .EQ. 2) .OR. (ITFD (ILINK) .EQ. 4)) THEN C $\star * \star * \star * \star \star \star \star \star \star * * *$ NORMAI AND LOGNORMAL DISTRIBUTIONS TLF $=$ XNORM (TFT $(1$, ILINK), TFT $(2$, ILINK))

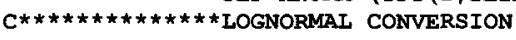
IF (ITED (ILINK) .EQ. 4) TLF=EXP (TLE) ENDIF

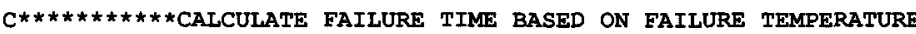

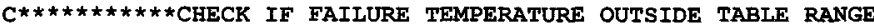
IF (TLF .LT. TH $(0$, ITHL(ILINK))) GO TO 9000

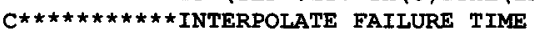

TIMLF $=0.0 D O$

DO 1000 ITIME=1, NTIME

IF (TH (ITIME, ITHL (ILINK)) ,GE. TLE) THEN

TIMLF=T (ITIME-1) + (T (ITIME) - T (ITIME-1)) *

(TLF - TH (ITIME-1, ITHL (ILINK)))

(TH (ITIME, ITHL (ILINK)) -

TH (ITIME-1, ITHL (ILINK)) )

GO TO 1100

$1000 \quad$ CONTINUE

GO TO 9000

$C \star \star \star \star \star \star \star \star \star \star \star \star \star$ SAVE MINIMUM AND MAXIMUM FAILURE TIMES

IF (CL (ILINK) (1:2) .EQ. 'SL') THEN

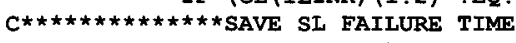

TFAIL (IIINK) $=$ T IML

$C \star \star \star \star \star \star \star \star \star \star \star \star \star \star \star \star$ SAVE MINIMUM SL FAILURE TIME

TSLFMN=MIN (TSLEMN， TIMLF 


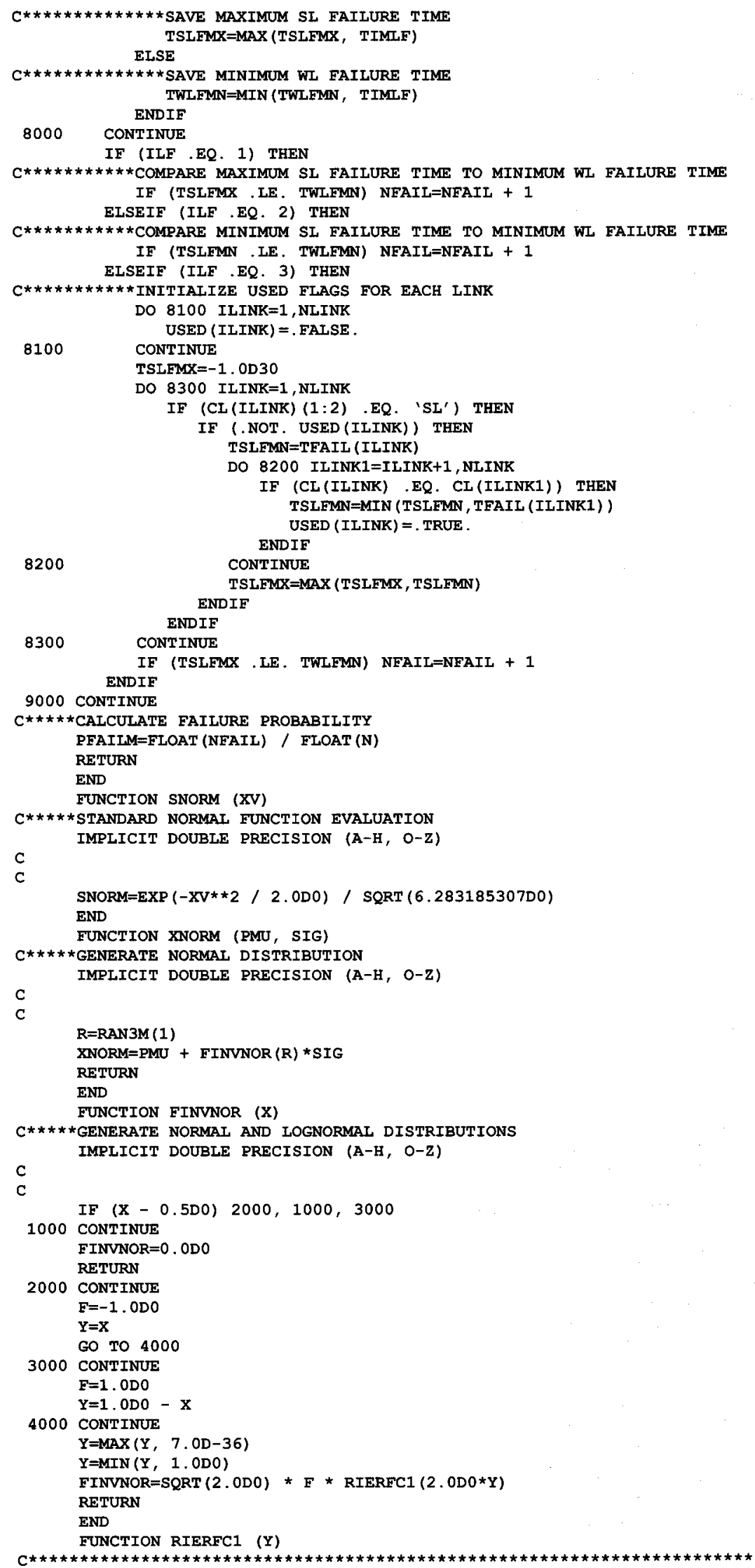


C FUNCTION RIERFC1 IS USED IN GENERATING THE NORMAL AND

C LOGNORMAI DISTRIBUTIONS

THIS IS THE SAME AS THE RIERFC ROUTINE IN THE DEAMOS LIBRARY THE NAME MODIFICATION WAS TO PREVENT OUR IIBRARY FROM CALLING ANOTHER LIBRARY. ELF OCTOBER 1980

WRITTEN BY D.E. AMOS AND S.L. DANIEL, SEPTEMBER, 1972.

REFERENCES

HASTINGS, C.JR., APPROXIMATIONS FOR DIGITAI COMPUTERS,

PRINCETON UNIVERSITY PRESS, PRINCETON, N.J., 1955

COMMUNICATION FROM L.F. SHAMPINE FOR CHEBYSHEV COEFEICIENTS.

ABSTRACT

RIERFC EVALUATES THE INVERSE COERROR FUNCTION DEFINED BY

$$
Y=\operatorname{ERFC}(X) \quad 0 \text {.LE. } X \cdot I T \text {. INFINITY }
$$

WHERE 0. LT.Y.LE.1. CHEBYSHEV APPROXIMATIONS ON

$\operatorname{EXP}(-81)$.LE, $Y$.LT. $0.1,0.1$.LE. $Y$.LT. $0.5,0.5$.LE. Y .LE. 1 .

ARE USED WITH A CHANGE OF VARIABLES

$\mathrm{YY}=\mathrm{C} 1 \star \mathrm{W}+\mathrm{C} 2, \mathrm{~W}=\mathrm{SQRT}(-\mathrm{LN}(\mathrm{Y})), \mathrm{YY}=5 . \star \mathrm{Y}-1.5, \mathrm{YY}=2 . \star(1 .-\mathrm{Y})$

RESPECTIVELY. THE INVERSE OF THE NORMAL DISTRIBUTION IS GIVEN BY

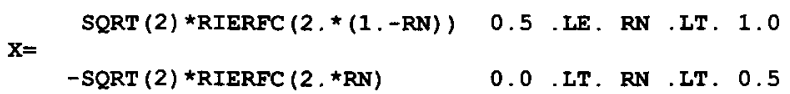

THE RELATIVE FRROR IN RIERFC DECRFASES FROM 1.E-10 TO 3.E-13

AS Y INCREASES FROM EXP (-81) TO 1.0 .

DESCRIPTION OF ARGUMENTS

INPUT

$\mathbf{Y} \quad-\mathbf{Y}, \operatorname{EXP}(-81)$.LE, Y.LE.1

OUTPUT

RIERFC - VALUE FOR THE INVERSE COERROR FUNCTION

ERROR CONDITIONS

Y.LT.EXP (-81) OR Y.GT.1 ARE FATAL ERRORS

IMPLICIT DOUBLE PRECISION (A-H, O-Z)

DIMENSION $A(22,3), A 1(22), A 2(22), A 3(22)$

EOUIVALENCE (A $(1,1), A 1(1))$

EQUIVALENCE (A $(1,2), A 2(1)$

EQUIVALENCE (A $(1,3), \mathrm{A} 3(1)$

DATA A1 /

$19.18725611735013 \mathrm{D}-01,0.0 \mathrm{DO}, 1.68792878000327 \mathrm{D}-02,0.0 \mathrm{DO}$,

$6.60337139058300 \mathrm{D}-04,0.0 \mathrm{DO}, 3.20203849839380 \mathrm{D}-05,0.0 \mathrm{DO}$,

$1.72060607522481 \mathrm{D}-06,0.0 \mathrm{DO}, 9.81965971588191 \mathrm{D}-08,0.0 \mathrm{DO}$,

$5.83049613537653 \mathrm{D}-09$, 0.0DO, 3.56019351836136D-10, 0.ODO,

2.21968915783128D-11, 0. ODO, 1.40639693109741D-12, 0.0DO, $9.02597345404862 \mathrm{D}-14,0.0 \mathrm{DO}$

DATA A2 / 1.54701109458613D+00, $-3.31460331083896 \mathrm{D}-01$, 4.33001124090060D-02, $-1.06564004165532 \mathrm{D}-02$,

$2.90613542304156 \mathrm{D}-03,-8.61872838022491 \mathrm{D}-04$

$2.67933751795053 \mathrm{D}-04,-8.60838893942933 \mathrm{D}-05$,

$2.83232058814598 \mathrm{D}-05,-9.48870819734494 \mathrm{D}-06$,

$3.22422655069385 \mathrm{D}-06,-1.10815778472076 \mathrm{D}-06$,

3.84464770797987D-07, -1.34439275565208D-07,

4.73255976052393D-08, $-1.67556011100019 \mathrm{D}-08$,

$5.96199003969093 \mathrm{D}-09,-2.13070503291886 \mathrm{D}-09$,

$7.64427040920545 \mathrm{D}-10,-2.75198005584737 \mathrm{D}-10$,

$9.93792246090789 \mathrm{D}-11,-3.59877382902119 \mathrm{D}-11 /$

DATA A3 / $1.10642888011036 \mathrm{D}+01,4.34299147561447 \mathrm{D}+00$

$-2.33781774969295 \mathrm{D}-02,4.23345215362947 \mathrm{D}-03$,

8.68757084192089D-06, $-5.98261113270881 \mathrm{D}-04$,

4.50490139240298D-04, $-2.54858131942102 \mathrm{D}-04$,

$1.27824189261340 \mathrm{D}-04,-5.97873878043957 \mathrm{D}-05$,

$2.66474012012582 \mathrm{D}-05,-1.14381836209267 \mathrm{D}-05$, 


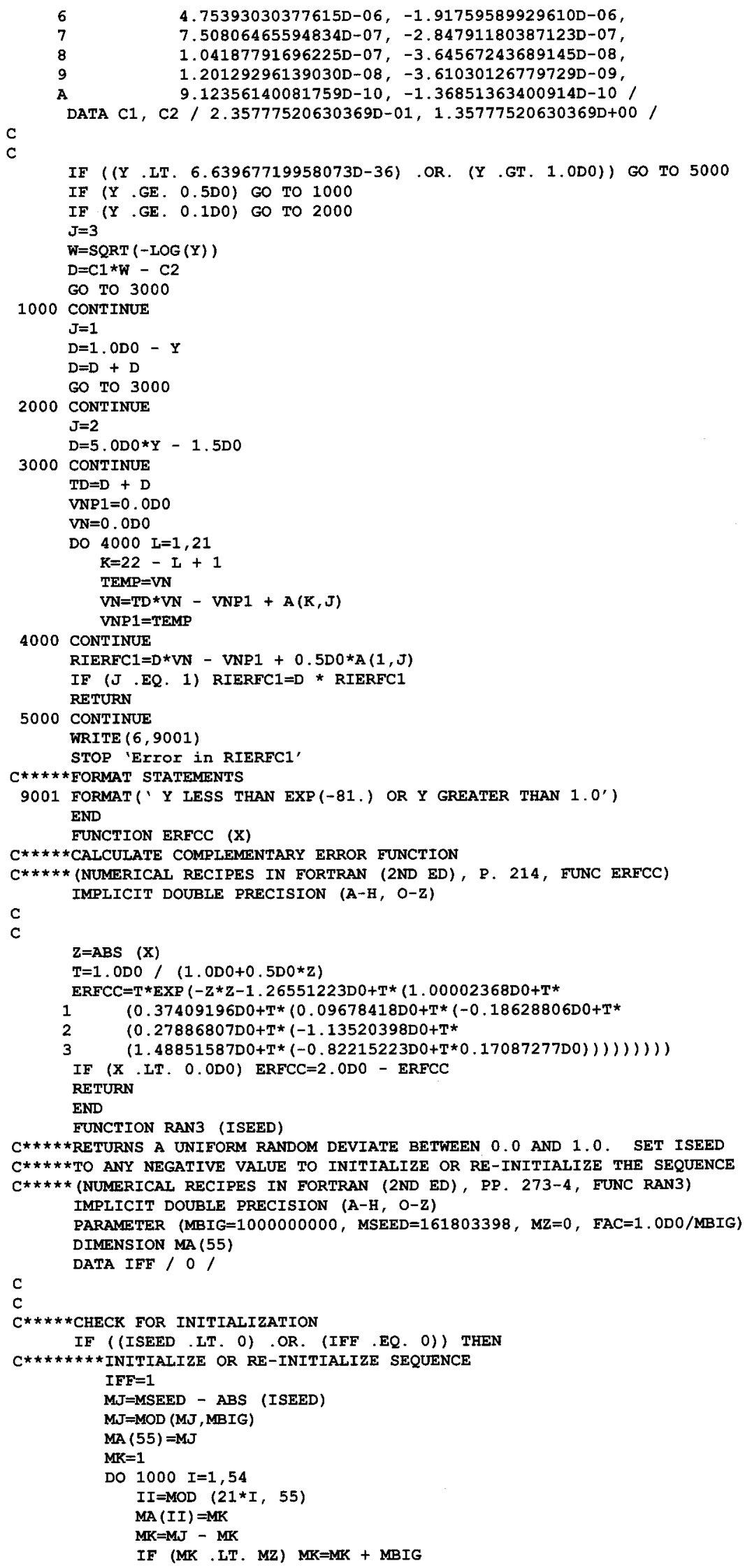




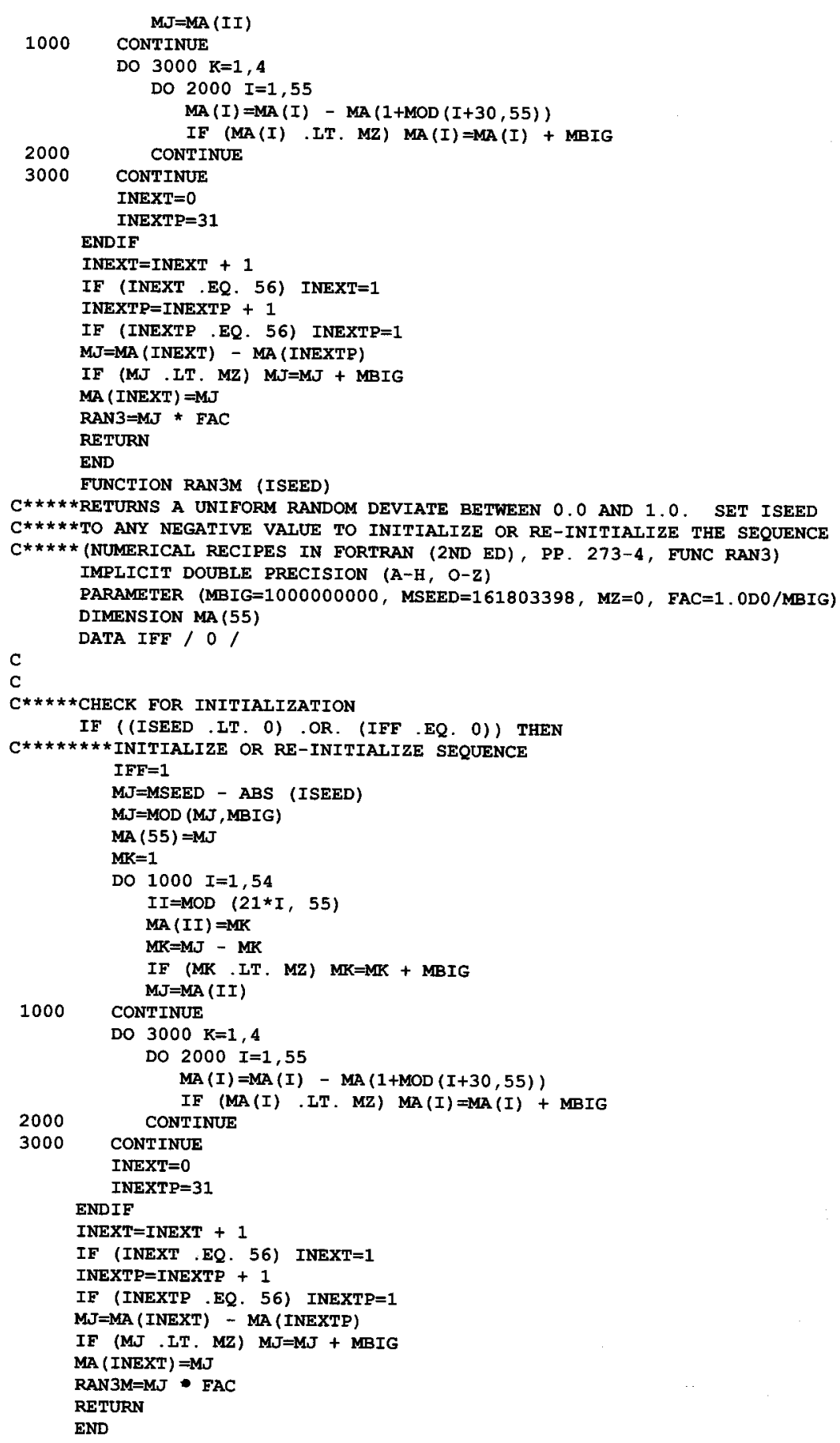




\section{DISTRIBUTION:}

\section{External Distribution}

Prof. Harish Agarwal

University of Notre Dame

Dept. of Aerospace \& Mechanical Engineering

Notre Dame, IN 46556

Prof. G. E. Apostolakis

Department of Nuclear Engineering

Massachusetts Institute of Technology

Cambridge, MA 02139-4307

Prof. Bilal Ayyub

University of Maryland

Center for Technology \& Systems Management

Civil \& Environmental Engineering

Rm. 0305 Martin Hall

College Park, MD 20742-3021

Prof. James Berger

Inst. of Statistics and Decision Science

Duke University

Box 90251

Durham, NC 27708-0251

Prof. Daniel Berleant

Iowa State University

Department of EE \& CE

2215 Coover Hall

Ames, IA 50014

Prof. V. M. Bier

Department of Industrial Engineering

University of Wisconsin

Madison, WI 53706

Kenneth T. Bogen

P.O. Box 808

Livermore, CA 94550

E. Bonano

Beta Corporation Int.

6613 Esther, NE

Albuquerque, NM 87109

Robert J. Budnitz

Future Resources Associates

2039 Shathck Avenue, Suite 402

Berkeley, CA 94704
Prof. Chun-Hung Chen (2)

Department of Systems Engineering \&

Operations Research

George Mason University

4400 University Drive, MS 4A6

Fairfax, VA 22030

Prof. W. J. Conover

College of Business Administration

Texas Tech. University

Lubbock, TX 79409

Prof. Allin Cornel1

Department of Civil and Environmental

Engineering

Terman Engineering Center

Stanford University

Stanford, CA 94305-4020

Prof. Alison Cullen

University of Washington

Box 353055

208 Parrington Hall

Seattle, WA 98195-3055

Department of Energy (5)

Attn: Kevin Greenaugh, NA-115

D. Kusnezov, NA-114

Jamileh Soudah, NA-114

K. Sturgess, NA-115

J. Van Fleet, NA-113

Forrestal Building

1000 Independence Ave., SW

Washington, DC 20585

Prof. U. M. Diwekar

Center for Energy and Environmental Studies

Carnegie Mellon University

Pittsburgh, PA 15213-3890

Prof. David Draper

Applied Math \& Statistics

147 J. Baskin Engineering Bldg.

University of California

1156 High St.

Santa Cruz, CA 95064

Pamela Doctor

Battelle Northwest

P.O. Box 999

Richland, WA 99352 
Prof. Isaac Elishakoff

Dept. of Mechanical Engineering

Florida Atlantic University

777 Glades Road

Boca Raton, FL 33431-0991

Scott Ferson

Applied Biomathematics

100 North Country Road

Setauket, New York 11733-1345

Prof. C. Frey

Department of Civil Engineering

Box 7908MCSU

Raleigh, NC 27659-7908

B. John Garrick

Garrick Consulting

923 SouthRiver Road, Suite 204

St. George, UT 84790-6801

Michael B. Gross

Michael Gross Enterprises

21 Tradewind Passage

Corte Madera, CA 94925

Prof. Ramana Grandhi (2)

Dept. of Mechanical and Materials Engineering

3640 Colonel Glenn Hwy.

Dayton, OH 45435-0001

Prof. Raphael Haftka

Dept. of Aerospace and Mechanical

Engineering and Engineering Science

P.O. Box 116250

University of Florida

Gainsville, F L 3261 1-6250

F. E. Haskin

901 Brazos Place, SE

Albuquerque, NM 87123

Prof. Richard Hills

New Mexico State University

College of Engineering, MSC 3449

P.O. Box 30001

Las Cruces, NM 88003

Prof. Steve Hora

Institute of Business and Economic Studies

University of Hawaii, Hilo

$523 \mathrm{~W}$. Lanikaula

Hilo, HI 96720-409 1
R.L. Iman

Southwest Design Consultants

12005 St. Mary's Drive, NE

Albuquerque, NM 87111

Prof. Alan Karr

Inst. of Statistics and Decision Science

Duke University

Box 90251

Durham, NC 27708-0251

Prof. W. E. Kastenberg

Department of Nuclear Engineering

University of California, Berkeley

Berkeley, CA 94720

Prof. George Klir

Binghamton University

Thomas J. Watson School of Engineering \&

Applied Sciences

Engineering Building, T-8

Binghamton NY 13902-6000

Prof. Vladik Kreinovich

University of Texas at El Paso

Computer Science Department

500 West University

El Paso, TX 79968

Prof. Sankaran Mahadevan

Vanderbilt University

Department of Civil and Environmental

Engineering

Box 6077 , Station B

Nashville, TN 37235

Prof. Max Morris (2)

Department of Statistics

Iowa State University

304A Snedecor-Hall

Ames, IW 50011-1210

Prof. Ali Mosleh

Center for Reliability Engineering

University of Maryland

College Park, MD 207 14-21 15

Naval Research Laboratory (2) Attn: Allen J. Goldberg Robert Gover

Cod 5753

4555 Overlook Avenue

S.W. Washington D.C. 20375 
Prof. Efstratios Nikolaidis (2)

MIME Dept.

4035 Nitschke Hall

University of Toledo

Toledo, OH 43606-3390

D. Warner North

North Works, Inc.

1002 Misty Lane

Belmont, C.A. 94002

Prof. David Okrent

Mechanical and Aerospace Engineering Department

University of California

48-121 Engineering IV Building

Los Angeles, CA 90095-1587

Dr. Gareth Parry

19805 Bodmer Ave

Poolesville, MD 200837

Prof. M. Elisabeth Paté-Cornel1

Department of Industrial Engineering and Management

Stanford University

Stanford, CA 94305

Prof. Herschel Rabitz

Princeton University

Department of Chemistry

Princeton, NJ 08544

Kadambi Rajagopal

The Boeing Company

6633 Canoga Avenue

Canoga Park, CA 91309-7922

Prof. John Renaud

Dept. of Aerospace \& Mechanical Engr.

University of Notre Dame

Notre Dame, IN 46556

Prof. Tim Ross (2)

Dept. of Civil Engineering

University of New Mexico

Albuquerque, NM 87131

Prof. J. Sacks

Inst. of Statistics and Decision Science

Duke University

Box 90251

Durham, NC 27708-0251
Southwest Research Institute (3)

Attn: L. Huyse

S. Mohanty

B.Thacker

P.O. Drawer 28510

620 Culebra Road

San Antonio, TX 78284

Martin Tierney

Plantae Research Associates

415 Camino Manzano

Santa Fe, NM 87505

Prof. T. G. Theofanous

Department of Chemical and Nuclear Engineering

University of California

Santa Barbara, CA 93106

Prof. Fulvio Tonon (2)

Geology and Geophysics Dept.

East Room 719

University of Utah

135 South 1460

Salt Lake City, UT 84112

Prof. Robert W. Walters (2)

Aerospace and Ocean Engineering

Virginia Tech

215 Randolph Hall, MS 203

Blacksburg, VA 24061-0203

Christopher G. Whipple

Environ

Marketplace Tower

6001 Shellmound St. Suite 700

Emeryville, C.A. 94608

Justin Y-T Wu

8540 Colonnade Center Drive, Ste 301

Raleigh, NC 27615

Prof. Ron Yager

Machine Intelligence Institute

Iona College

715 North Avenue

New Rochelle, NY 10801

\section{Foreign Distribution}

Prof. Yakov Ben-Haim (2)

Department of Mechanical Engineering Technion-Israel Institute of Technology Haifa 32000

ISRAEL 
Prof. Gert de Cooman (2)

Universiteit Gent

Onderzoeksgroep, SYSTeMS

Technologiepark - Zwijnaarde 9

9052 Zwijnaarde

BELGIUM

A.P. Bourgeat

Équip Analyse Numérique

Faculté des Sciences

42023 St. Entienne Cedex 2

FRANCE

Prof. Russell Cheng

University of Kent at Canterbury

Comwallis Building

Canterbury, Kent, CT2 7NF

UNITED KINGDOM

Prof. Roger Cooke

Department of Mathematics

Delft University of Technology

P.O. Box 50312800 GA Delft

THE NETHERLANDS

European Commission (4)

Attn: Francesca Campolongo

Mauro Ciechetti

Andrea Saltelli

Stefano Tarantola

JRC Ispra, ISIS

21020 Ispra

ITALY

Prof. Thomas Fetz

University of Innsbruck

Technikerstr 13

Inmbruck AUSTRIA 6020

Forshunginstitute GRS (2)

Attn: Eduard Hofer

B. Kryzkacz-Hausmann

Forschungsgelande Nebau 2

85748 Garching

GERMANY

Forschungszentrum Karlsruhe (2)

Attn: F. Fischer

J. Ehrhardt

Inst. Kern \& Energietechn

Postfach 3640, D-76021

Karlsruhe

GERMANY
Prof. Simon French

School of Informatics

University of Manchester

Coupland 1

Manchester M13 9pl

UNITED KINGDOM

Prof. Jim Hall

University of Bristol

Department of Civil Engineering

Queens Building, University Walk

Bristol UK 8581TR

Prof. Igor Kozine (2)

Systems Analysis Department

Riso National Laboratory

P. O. Box 49

DK-4000 Roskilde

DENMARK

J. Jaffré

INRIA - Roquencourt

B.P. 105

78153 Le Chesnay Cedex

FRANCE

Prof. J.P.C. Kleijnen

Department of Information Systems

Tilburg University

5000 LE Tilburg

THE NETHERLANDS

Prof. Michael Oberguggenberger

University of Innsbruck

- Technikerstr 13

Innsbruck AUSTRIA 6020

Professor A. O'Hagan

Department of Probability and Statistics

University of Sheffield

Hicks Building

Sheffield S3 7RH

UNITED KINGDOM

Prof. I. Papazoglou

Institute of Nuclear Technology-Radiation Protection

N.C.S.R. Demolaitos

Agha Papakevi

153-10 Athens

GREECE 
Prof. Marian Scott

Department of Statistics

University of Glasgow

Glasgow G12 BQW

UNITED KINGDOM

Prof. Ilya Sobol'

Russian Academy of Sciences

Miusskaya Square

125047 Moscow

RUSSIA

Prof. Tamas Turanyi

Eotvos University (ELTE)

P.O. Box 32

1518 Budapest

HUNGARY

Prof. Enrico Zio

Politecnico di Milano

Via Ponzia 3413

20133 Milan

ITALY

Prof. Lev Utkin

Institute of Statistics

Munich University

Ludwigstr. 33

80539, Munich

GERMANY

\section{Department of Energy Laboratories}

Los Alamos National Laboratory (13)

Mail Station 5000

P.O. Box 1663

Los Alamos, NM 87545

Attn: Mark C. Anderson, MS T080

Jane M. Booker, MS P946

Terrence Bott, MS K557

Katherine Campbell, MS F600

Scott Doebling, MS P946

S. Eisenhawer, MS K557

Cliff Joslyn, MS B265

F. Hemez, MS P946

M. Hyman, MS B284

H. Martz, MSF600

S. Keller-McNulty, MS F600

Kari Sentz, MS F600

Alyson G. Wilson, MS F600

Lawrence Livermore National Laboratory (4)

7000 East Ave.

P.O. Box 808

Livermore, CA 94550
Attn: Henry Hsieh, MS L-229

Richard Klein, MS L-023

Roger Logan, MS L-125

Cynthia Nitta, MS L-096

Argonne National Laboratory (2)

Attn: Paul Hovland

Mike Minkoff

MCS Division

Bldg. 221, Rm. C-236

9700 S. Cass Ave.

Argonne, IL 60439

\section{Sandia Internal Distribution}

$\begin{array}{llll} & \text { MS } & \text { Org } & \\ 1 & 0429 & 2100 & \text { J. S. Rottler } \\ 1 & 0437 & 2100 & \text { R. C. Hartwig } \\ 1 & 0447 & 2111 & \text { P. Davis } \\ 1 & 0479 & 2113 & \text { J. O. Harrison } \\ 1 & 0453 & 2130 & \text { H. J. Abeyta } \\ 1 & 0481 & 2132 & \text { M. A. Rosenthal } \\ 1 & 0427 & 2134 & \text { R. A. Paulsen } \\ 1 & 0509 & 2300 & \text { M. W. Callahan } \\ 1 & 0512 & 2500 & \text { T. E. Blejwas } \\ 1 & 1115 & 4200 & \text { D. S. Miyoshi } \\ 1 & 1138 & 6222 & \text { S. M. DeLand } \\ 1 & 0771 & 6800 & \text { D.L. Berry } \\ 1 & 1395 & 6821 & \text { D. Kessel } \\ 1 & 1395 & 6821 & \text { J. W. Garner } \\ 1 & 0778 & 6851 & \text { R. MacKinnon } \\ 1 & 0778 & 6851 & \text { P. Swift } \\ 1 & 0776 & 6852 & \text { M. K. Knowles } \\ 1 & 0776 & 6852 & \text { C. Sallaberry } \\ 1 & 1399 & 6853 & \text { M. G. Marietta } \\ 1 & 1399 & 6853 & \text { D. Sevougian } \\ 1 & 0776 & 6853 & \text { R. P. Rechard } \\ 1 & 0748 & 6861 & \text { M. Allan } \\ 1 & 0748 & 6862 & \text { B. Waters } \\ 1 & 0771 & 6870 & \text { J.E. Kelly } \\ 1 & 0779 & 6874 & \text { H.-N. Jow } \\ 1 & 0779 & 6874 & \text { J.D. Johnson } \\ 1 & 9004 & 8100 & \text { R. H. Stulen } \\ 1 & 9007 & 8200 & \text { D. R. Henson } \\ 1 & 9014 & 8242 & \text { D. M. Kwon } \\ 1 & 9003 & 8900 & \text { K. E. Washington } \\ 1 & 9003 & 8940 & \text { C. M. Hartwig } \\ 1 & 9003 & 8960 & \text { J. L. Handrock } \\ 1 & 0384 & 9100 & \text { T. C. Bickel } \\ 1 & 1393 & 9100 & \text { T.Y. Chu } \\ 1 & 0825 & 9110 & \text { W. Hermina } \\ 1 & 0834 & 9112 & \text { J. E. Johannes } \\ 1 & 1310 & 9113 & \text { S. N. Kempka } \\ 1 & 0825 & 9115 & \text { B. Hassan } \\ 1 & 0836 & 9116 & \text { E. S. Hertel } \\ & & & \end{array}$




$\begin{array}{llll}1 & 0836 & 9116 & \text { D. Dobranich } \\ 1 & 0139 & 9120 & \text { P. J. Wilson } \\ 1 & 0555 & 9122 & \text { M. S. Garrett } \\ 1 & 0893 & 9123 & \text { J. Pott } \\ 1 & 0847 & 9124 & \text { J. M. Redmond } \\ 1 & 0557 & 9125 & \text { T. J. Baca } \\ 1 & 0372 & 9126 & \text { R. A. May } \\ 1 & 0372 & 9127 & \text { J. Jung } \\ 1 & 0824 & 9130 & \text { J. L. Moya } \\ 1 & 0821 & 9132 & \text { L. A. Gritzo } \\ 1 & 1135 & 9132 & \text { J. T. Nakos } \\ 1 & 0836 & 9132 & \text { C. Romero } \\ 1 & 1135 & 9132 & \text { S. R. Tieszen } \\ 3 & 0828 & 9133 & \text { M. Pilch } \\ 1 & 0828 & 9133 & \text { K. J. Dowding } \\ 1 & 0828 & 9133 & \text { A. A. Giunta } \\ 25 & 0779 & 9133 & \text { J. C. Helton } \\ 25 & 0828 & 9133 & \text { W. L. Oberkampf } \\ 1 & 0828 & 9133 & \text { J. R. Red-Horse } \\ 1 & 0828 & 9133 & \text { V. J. Romero } \\ 1 & 0828 & 9133 & \text { M. P. Sherman } \\ 1 & 1135 & 9134 & \text { S. Heffelfinger } \\ 1 & 0384 & 9140 & \text { H. S. Morgan } \\ 1 & 0382 & 9141 & \text { E. A. Boucheron } \\ 1 & 0380 & 9142 & \text { K. F. Alvin }\end{array}$

$\begin{array}{llll}1 & 0382 & 9143 & \text { J. R. Stewart } \\ 1 & 0370 & 9211 & \text { S. A. Mitchell } \\ 1 & 0370 & 9211 & \text { M. S. Eldred } \\ 1 & 0370 & 9211 & \text { L. P. Swiler } \\ 1 & 0370 & 9211 & \text { T. G. Trucano } \\ 1 & 0316 & 9230 & \text { P. Yarrington } \\ 1 & 0139 & 9900 & \text { M. O. Vahle } \\ 1 & 0139 & 9904 & \text { R. K. Thomas } \\ 1 & 0139 & 9905 & \text { S. E. Lott } \\ 1 & 0428 & 12300 & \text { D. D. Carlson } \\ 1 & 0428 & 12301 & \text { V. J. Johnson } \\ 1 & 0829 & 12337 & \text { J. M. Sjulin } \\ 1 & 0829 & 12337 & \text { B. M. Rutherford } \\ 1 & 0829 & 12337 & \text { F. W. Spencer } \\ 5 & 0405 & 12346 & \text { T. R. Jones } \\ 1 & 0405 & 12346 & \text { T. D. Brown } \\ 1 & 0405 & 12346 & \text { S. E. Camp } \\ 1 & 0830 & 12335 & \text { K. V. Diegert } \\ 1 & 1170 & 15310 & \text { R. D. Skocypec } \\ 1 & 1176 & 15312 & \text { D. J. Anderson } \\ 1 & 1176 & 15313 & \text { R. M. Cranwell } \\ 1 & 9018 & 8945-1 & \text { Central Technical } \\ & & \text { Files } & \\ 2 & 0899 & 9616 & \text { Technical Library }\end{array}$

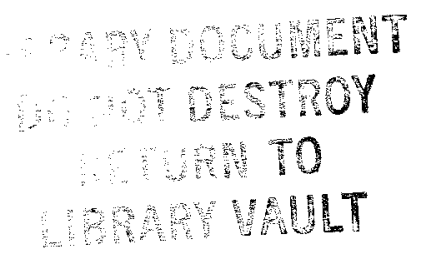

